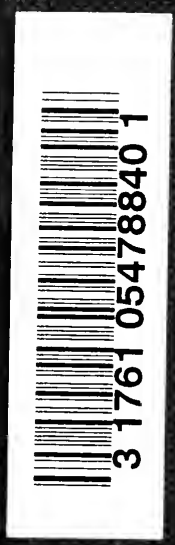





THE THERMODYNAMICS OF HEAT-ENGINES 


$$
\text { The > X }
$$




\section{THE THERMODYNAMICS}

\section{OF HEAT-ENGINES}

BY

SIDNEY A. REEVE

PROFESSOR OF STEAM-ENGINEERING AT THE WORCESTER

POLYTECHNIC INSTITUTE

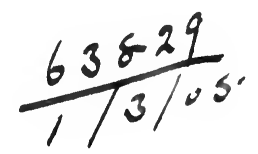

Xrew Hark

THE MACMILLAN COMPANY

LONDON: MACMILLAN \& CO., LTD.

1903 
Copyright, 1903,

BY THE MACMILLAN COMPANY.

Set up and electrotyped January, 1903. 


\section{To ftly $\mathfrak{U} \mathfrak{U} \mathfrak{i f e}$}

TO WHOSE DEVOTION AND AID

(ALTHOUGH SHE DOESN'T KNOW ENTROPY FROM CARBONIC ACID)

THE EXISTENCE OF THIS BOOK IS DUE

IT IS DEDICATED 
Digitized by the Internet Archive in 2007 with funding from Microsoft Corporation 


\section{CONTENTS}

\section{PART I}

\section{THEORY}

\section{CHAPTER I}

The General Principles of Energetics .

Definitions. The Forms of Energy. Their Interchangeability. The Law underlying Such Changes. The Constancy of Energy. The First Law of Energetics. The First Law of Thermodynamics.

The Dimensions of Energy. Classification. The Direction of Energy-transformation. The Second Law of Energetics. The Second Law of Thermodynamics.

\section{CHAPTER II}

\section{The Cycle}

Definitions. The Conditions Essential to the Existence of Cycles. The Rectangular Cycle in Mechanical Energies. The Rectangular Cycle in Thermodynamic Energies. The Force-distance and Entropytemperature Diagrams. Isotherms and Adiabatics. The Isomorphic Curve. Efficiencies. The Reversible Cycle. The Refrigerating Cycle.

\section{CHAPTER III}

The Thermal Properties of Matter

The Molecular Hypothesis. Molecular Energies. Solids, Liquids, and Gases. Specific Heat. Latent Heats. Temperature-heat. Disgregation-energy and External Work. The Absolute Zero. The Portrayal of Thermal Processes by the Entropy-temperature Diagram. The Formation of Steam under Constant Pressure. 


\section{CHAPTER IV}

The Steam-engine Cycle.

The Triangular Cycle of the Boiler-explosion. The Quadrilateral Cycle of the Perfect Steam-engine, or the Rankine Cycle. Modifications due to Wet Steam and to Superheat. Effect of Exhaust into a Vacuum. The Cycle of the Non-expansive Steam-engine, or of the Direct-acting Steam-pump. The Standard Steam-engine Cycle, with Partial Expansion. The Carnot Cycle with Steam as a Working-substance.

\section{CHAPTER V}

The Laws of the Permanent Gases

Boyle's Law. Charles's Law. The Two combined. The Natural Significance of the Constant. Expansion-curves in the Pressurevolume Diagram. Their Equations. The Work done by them. Alterations in Temperature. The Compression and Expansion of Air.

\section{CHAPTER VI}

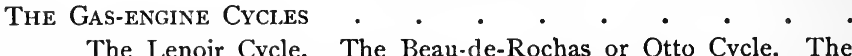
Joule Cycle. The Natural Relations between the Three. Efficiencies and Capacities.

\section{CHAPTER VII}

The Hot-Air Engines.

The Stirling Cycle. The Ericsson Cycle. The Carnot Cycle with Air as a Working-substance. Efficiencies, Capacities, and Possibilities.

\section{CHAPTER VIII}

Comparative Summary of Heat-engine Possibilities . ' • . 164

\section{CHAPTER IX}

The Refrigerating Machines

The Cycle of the Air-refrigerating Machine, or the Reversed Joule Cycle. The Cycle of the Ammonia Compression Machine. The Kelvin Warming-machine. 


\section{PART II}

\section{APPLICATION TO PRACTICE}

\section{CHAPTER I}

The Simple Steam-engine .

Projected Indicator-cards. Elementary Calculations. Expansionratios and Clearance.

Thermal Modifications of the Theoretic Cycle in the Actual Cylinder. Condensation and Reëvaporation. Factors affecting it. Speed. Size. Ratio of Expansion. Steam-jacketing. Superheat. The Observation and Analysis by the Entropy-temperature Method of the Cycle followed by the Actual Engine. Efficiencies.

Resultant Types of Steam-engine. The Single-valve, Fixedeccentric, Throttle-governed Engine. The Single-valve Shaftgoverned Engine. The Four-valve Type. Comparison of Results and of Proper Fields of Service.

\section{CHAPTER II}

The Compound Steam-engine

Mechanical Considerations. Thermodynamic Considerations. Cylinder-ratios. Woolf and Receiver Types. Methods of Governing.

\section{CHAPTER III}

The Otto Gas-engine .

Modifications of the Theoretic Cycle in the Actual Cylinder. The Effect of the Water-jacket. The Effect of Delayed Combustion. The Effect of the Cylinder-walls on the Expansion-curve. Similarity of the Thermal Phenomena in Gas-engines and Steam-engines. Scavenging.

The Observation and Analysis by the Entropy-temperature Method of the Cycle followed by the Actual Gas-engine. 



\section{NOTATION}

$C=$ the density-constant for a permanent gas, or the number of foot-pounds of external work done during the heating of one pound of the gas one degree Fahrenheit under constant pressure. For air, $C=53.18$.

$C^{\prime}=$ the specific density-constant involved in a case concerning a mass of gas other than one pound.

$D=$ the disgregation-energy of dry saturated steam.

$E=$ quantity of energy.

$F=$ efficiency.

$H=$ the total heat of dry saturated steam above that of melted ice.

$I=$ internal energy of a gas or vapor.

$J=$ the mechanical equivalent of heat $=778$.

$K=$ the ratio of the specific heats of a gas under constant pressure and constant volume respectively.

$L=$ the tatent heat of vaporization of dry saturated steam.

$l=$ the isomorphic heat of water above that of melted ice.

$M=$ mass.

$N=$ entropy.

$P=$ pressure, in pounds per square inch unless otherwise stated.

$Q=$ quantity of heat.

$Q_{1}=$ the heat supplied at the upper temperature-level during a cycle.

$Q_{2}=$ the heat rejected at the lower temperature-level of a cycle.

$Q_{w}=$ the heat available for doing work $=Q_{1}-Q_{2}$.

$q_{w}=$ the heat actually converted into work during a cycle.

$R=$ volume-ratio of gaseous expansion.

$S=$ specific heat.

$T=$ absolute temperature Fahrenheit.

$T_{1}=$ the upper absolute temperature of a pair of temperature-limits.

$T_{2}=$ the lower absolute temperature of a pair of temperature-limits.

$t=$ temperature Fahrenheit.

$V=$ volume, in cubic feet unless otherwise stated.

$W=$ weight, in pounds avoirdupois unless otherwise stated.

$X=$ the external work involved in the vaporization of dry saturated steam.

$x=$ the unknown or indefinite exponent of the volume-ratio (in the expansion of a gas) in the equation connecting it with the pressure-ratio. $y=$ the unknown fractional proportion of steam to total mass in a steamand-water mixture. 

PART I

THEORY 



\section{THE THERMODYNAMICS OF HEAT-ENGINES}

\section{$\rightarrow$ \\ CHAPTER I \\ THE GENERAL PRINCIPLES OF ENERGETICS}

THE term thermodynamics is derived from two Greek

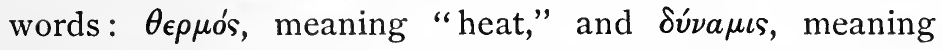
"force." It refers to the study of the relations between heat and mechanical energy.

There is scarcely a science so widespread in its applications as thermodynamics. To the student of modern science the source of all things is heat. Wind and weather, all vegetation, all life, take their origin and find their strength in heat. Not only man and all of the higher forms of animal life, but the lowest and simplest of vegetable types as well, depend upon heat for their existence. and absorb it as constantly as their food. This heatenergy it is the continuous duty of their lives to convert into mechanical motion, or work. In inanimate nature the same is true. The vigor of the storm, the irresistible motion of the glacier, the erosion of mountain's and the filling of valley or lake; the frosted window-pane, the sculptured iceberg, the mirage of the desert, the singing bullet, and the quiet beneficent storage of water in pond and reservoir ; 
the gun, the rocket, the steam-engine and the gas-engine, - all are manifestations of heat and its transformations into motion, all are more or less thermodynamic in character. For the study of each a comprehension of the principles of thermodynamics is necessary. Each is an illustration of the working of those principles and of the laws which limit them.

- The two quantities which form the stock-in-trade of all heat-engines - the raw material and the manufactured product, so to speak - are heat and mechanical energy. Both of these quantities belong under a common classification title as being both of them forms of energy.

According to the modern scientific analysis of natural phenomena, the entire universe is made up of two broad subdivisions of existence - matter and energy. The conception of matter is familiar to all. It apparently appeals directly to our senses at every turn. It apparently, too, appears in a myriad of different forms. The study of chemistry, however, teaches that in all of these infinitely varying forms there are really to be found only some eighty or ninety distinct kinds, or elements, of matter, such as carbon, hydrogen, calcium, etc. Physics, again, tells us that each of these elements, and each of their innumerable chemical combinations, exists in four distinct conditions: solid, liquid, gaseous, and radiant forms of matter.

The fundamental law which underlies both chemistry and physics is that of the Conservation of Matter. This law is the statement of the fact that, no matter what physical alterations matter may undergo, whether from solid to liquid or from liquid to gaseous, or the reverse, and no matter what chemical transformation may occur, whether the substance be burnt as fuel in a furnace, decomposed as lime in a kiln, or corroded with acids, throughout every 
conceivable change in its form and characteristics the quantity of matter in the universe remains always the same. Man may change its locality at will. Working in harmony with the laws which limit all natural action, he can transmute its outward appearance in a myriad of different ways, to suit almost every imaginable whim or purpose. But to alter in the slightest degree the existing quantity of matter, to create one particle, or to destroy a single atom, he has always been and always will be impotent. The universe is eternal. In the face of its steadfast continuity man's momentary existence and evanescent will are as cloud-wreaths against a mountain-side.

The other of the two broad subdivisions of all things natural includes the various forms of energy. These forms of energy are distinguished by external characteristics upon which is based a proper classification. But this classification is not parallel with that of either chemistry or physics. Whereas, so far as the science of chemistry has yet progressed with certainty, the various chemical elements can never be confused with one another, the several forms of energy are separated by lines often quite indistinct to our limited discernment. Whereas one chemical element can never be altered into any other, the various forms of energy are each of them capable of being transformed, under favorable conditions, into any one of the other forms. Nevertheless, under and through all study of any of the forms of energy, or of their mutual transformations, runs one fundamental and immutable law, that of the Conservation of Energy, quite parallel to the law of the conservation of matter. It states that, no matter what transformations may occur to any form of energy, the quantity of energy in the universe after the change is quite the same as it was before. These transformations may greatly alter its appearance to our senses, or its effect upon other things ; 
yet the rule remains unbroken. No energy has been destroyed and none created. Energy, the consort of matter in that union of all things which we call Nature, is eternal, is constant, as is matter.

These two laws, of the conservation of matter and of energy, together constitute the foundation of modern engineering science. It was ignorance of the law of the conservation of matter which led the alchemists of old on their long, costly, hopeless search for the "philosopher's stone," by mere touch of which all matter might be transmuted into gold. It is ignorance of the law of the conservation of energy which has more recently led to the strenuous, fascinating, but hopeless endeavor for the production of "perpetual motion," or mechanical energy produced without the aid of any anterior supply of energy. Volumes of history could be crowded with the list of the lives ruined or lost in the following of these two dreams, and no more forcible illustration could be adduced to prove the indispensable need of every engineer for a complete and accurate grasp of these two laws and of their absolute rigidity.

What is meant by the words energy and transformation? Energy is defined as "the ability to overcome resistance." All natural phenomena consist, in one way or another, of an overcoming of resistance; therefore all phenomena known to man are manifestations, in one way or another, of energy. It is a quality inherent in all matter, to some extent.

Yet, so far as we now know, any or all of the energy inherent in any body is quite distinct and entirely detachable from that body. Although human experience has never covered the case of a bit of matter possessing no energy, yet we always conceive of such a case as quite 
possible, because some portion of the energy of a mass of matter is always removable from it; nor is there any definite limit to the amount removable. Thus, a weight lifted above the earth possesses energy, for it is able to overcome resistance in falling again toward the earth. Yet the energy resident in the body of the weight is quite distinct from the matter composing the body; for if the weight be removed to a locality where it no longer possesses weight, say to the centre of the earth, the mass of the body will remain unchanged, while the energy has entirely disappeared. Similarly, a moving projectile possesses the ability to overcome resistance, and therefore contains energy; but when it has been brought to rest, if not too suddenly, the matter will remain unchanged, while the energy is gone.

The overcoming of resistance involves two distinct factors: (I) the resistance, and (2) the extent to which it is overcome. The first is most simply defined in terms of force, a thing so familiar to us all that no further analysis or definition of it need here be attempted. It does need a unit of measurement, however, and for this will be exclusively adopted, in the following pages, the pound avoirdupois. Secondly, the extent or distance through which the overcoming of resistance is effective is another matter of familiar experience and needs no definition. The unit for its measurement which will be used hereafter is the English foot.

It is next to be noted that the overcoming of resistance involves the expenditure of energy. The distance traversed by the acting force in the direction of its exertion is the same as that through which the resistance is overcome in a direction opposite to its exertion. This is the modern equivalent to saying that "action and reaction are equal and in the opposite direction." The driver must lose as 
much energy as the driven gains, and the driven must gain as much as the driver loses. The distinction between loss and gain is an artificial one and depends solely upon the point of view from which they are regarded; they are, therefore, properly distinguished by positive and negative signs, it being usual to consider the acting force as positive and the absorbing resistance as negative.

From the foregoing considerations is reached the fundamental idea that the unit of quantity of energy is that manifested when a force of one pound overcomes a resistance of one pound through a distance of one foot, during which process the acting force has expended and the resistance has absorbed one foot-pound of energy.

Work. - As this particular form of energy has its individual name, in the classification of different forms of energy, and is known as mechanical energy, or work, the foot-pound is also the unit for measuring quantities of work, the word work being understood in its mechanical sense. Further, as mechanical energy has always been the most familiar of all of the different forms of energy and the one with which acquaintance is first made, it has become the custom to base the units of the other forms of energy used by the engineer upon the foot-pound, and to define them in terms of it as the fundamental unit.

Power. - Closely associated with the idea of mechanical energy is that of power. Power is defined as the rate at which work is done. It therefore involves, besides the factors of force and distance which are involved in the idea of work, the additional factor of time. The unit of power is the horse-power, which is defined as that rate of doing work which will accomplish 33,000 foot-pounds of work if steadily continued for the space of one minute. This same rate would accomplish 550 foot-pounds of work in one second; so the latter is often given as a 
definition of the horse-power. In metrical units the horsepower is defined as the rate which would accomplish seventy-five kilogram-meters of work if continued for one second.

It is obvious that power, or the rate at which work is done, has nothing to do with the quantity of work accomplished. A very slow rate may accomplish a great deal of work, if it only keeps at it long enough, while a very high rate may be represented by a very small quantity of work, provided it be expended in a sufficiently short space of time. Thus, a pump driven by a motor having a power of much less than one horse-power, by working steadily, day and night, for weeks and months, may lift millions of tons of water and thereby perform tens of millions of footpounds of work; yet its power is none the less a fraction of a horse-power. Or, on the other hand, a rifle, accomplishing, in imparting motion to the bullet, no more work than does a boy in throwing a heavy stone, by performing the task in an exceedingly short period of time actually does exert a power, or a rate of performance, of something like 5000 horse-power.

The Forms of Energy. - Energy, the eternal, manifests itself to the human senses in several distinct aspects, or forms. Familiar instances are those of mechanical motion, light, sound, electricity, heat, etc. In fact, there is no phenomenon under the canopy of heaven which may be defined as constituting a process, as being aught else than a dead static fact, or condition, which is not a manifestation of energy, in some form or another. The motion of the sun and its planets, the rise of the tide, the shower of rain, the flash of lightning and the peal of thunder, the growth of vegetation and the most intricate processes of animal life, are all to be classed, in their broadest sense, 
as manifestations of energy; and all of them must live within its laws.

This myriad of different forms of energy may be subdivided into groups or classes in a variety of ways, each based upon some line of distinguishing characteristics. Of these the broadest classification is that into three main groups, viz.: Kinetic, potential, and vibratory forms of energy.

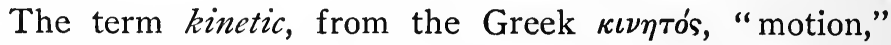
always implies the presence of motion. Hence, kinetic energy may be defined as that involved in the motion of a mass. It is one of the primary conceptions of mechanics that energy is always required in order to impart motion or change of motion to mass; and, obversely, that mass can never part with its motion except in the course of overcoming resistance. As motion without or separate from mass is a purely geometric conception, finding no counterpart in human experience, it will be sufficient, hereafter, to refer to kinetic energy simply as consisting of motion. The most familiar instances of kinetic energy are those of a moving projectile, of a hammer, or of a fly-wheel.

The term potential, from the Latin potior, "to be able," always implies latent ability. It is, however, the distinguishing characteristic of potential energy that it has always the appearance of having been acccumulated by previous motion, or kinetic energy. It will therefore be most clearly defined to the mind as stored motion. This fact may be easily fixed in the mind by association of the words potential and position; for potential energy is always involved in mere position, attained as the result of motion, as contrasted with kinetic energy or the motion which gave it birth.

To illustrate, the most familiar instance of potential energy is that of a weight raised from the earth. By rea- 
son of its separation from the earth it is able to overcome resistance in again falling into contact with the earth. The ability to do work is there, latent, unasserted; yet it is none the less powerful. Thus, imagine a six-inch rifle aimed vertically into the air and fired. The projectile, as it leaves the muzzle, possesses an enormous supply of energy which it has received from the explosion of the powder. As it arises, this kinetic energy is gradually and constantly being expended in overcoming the force, or resistance, of gravitation. The projectile must finally reach an elevation at which, its supply of kinetic energy having been all expended, it will have come to rest.

Let us suppose that a scaffolding had been built up from the earth to this point and that a man standing upon it should catch the projectile, at the instant of its stop, and hold it there. The projectile must contain at this instant, if we neglect the friction of the atmosphere, the entire amount of energy with which it left the gun; for if it be released, it will exert, in its fall, exactly that amount and will reach the earth with as high and destructive a velocity as it had at the start. But at the start this energy would have blown the man to atoms had he been so unfortunate as to come into contact with the projectile. At the top he may handle the deadly thing with entire impunity.

What is the difference? It is not easily to be explained, but a broad fact it certainly is, one which may not yet be stated as an absolute law but which is certainly more than a widespread coincidence, that the human organism is extremely sensitive to kinetic forms of energy but takes almost no cognizance, except through the medium of deductive reasoning, of potential forms. The distinction is important, even in the elementary study of engineering problems, because there is no one source of engineering failure, or of commercial, economic, and political failure, 
for that matter, so common as a lack of proper estimate of the possibilities concealed within the latent, invisible, potential forms of energy.

Of the third group of energy-forms, the vibratory energies, little may be said here, in spite of the fact that they are the most widespread and familiar of all the forms. Vibratory energy consists of periodic alternations between kinetic and potential forms of the same kind. The simplest instance is that of the pendulum. Here, supposing the pendulum to be already in swing, is visible at the centre of its arc the kinetic energy of the mass of the pendulumbob. This carries it up the one side of its arc, the kinetic energy being gradually transformed into the potential form of separation from the earth, until the extreme height of swing is reached. Here the transformation is complete and the energy all potential in form. But it does not remain so a single instant. The bob has no sooner come to rest than it starts upon its return-swing, reconverting its potential energy into motion until the lowest point of the arc be reached, when the process is repeated up the other side.

Here the alternations between the two forms is slow enough, usually, so that the eye, or the ear, as in the case of a clock, might easily distinguish the separate beats and count them. But if we substitute for the force of gravity the elasticity of a spring, and make the pendulum quite short, the rapidity of the beats may easily elude, the eye. The apparatus becomes blurred in appearance, and we know the rapidity of vibration only as a degree of smoothness of blur. If the vibrations be well above sixteen to the second, the ear can no longer count the separate sounds emitted by the pendulum in passing the centre; they become blurred into a continuous sound, or note, and the rapidity of vibration is known only as pitch. Yet each 
vibration is none the less a complete cycle of alternate transformations of kinetic into potential energy, of potential back into kinetic.

If we substitute for the gravity-pendulum an absolutely fluid mass, to which water is a close simile, we shall find - that each particle of water near the surface is easily set moving in a vertical circular orbit which will carry it alternately above and below the mean level of static equilibrium. Its extremes above and below, the crests and troughs respectively of the water-wave, correspond to the extremes of the pendulum's swing, its energy there being all potential in form, either positive or negative. Its alternations between the two constitute a new form of vibratory energy. Here, however, we find a strange and unwavering tendency to transmit the energy from one particle to another, and that almost without loss, so that the form of the vibration, or wave, as it is called, progresses continuously from one locality of the surface of the fluid mass to another. This is a faculty possessed not at all, or very slightly, by the vibratory motions of single solid bodies, such as the pendulum. It is a property of all fluids, however, and of all elastic, continuous solids, so far as transmission within their own confines is concerned. In air, which is elastic as well as fluid, these (or very similar) waves are called sound.

If we substitute for these well-known substances the less familiar ones of the luminiferous ether and the electric atom, the vibrations will take the form of light or electricity. If it be light, the number of vibrations, always numbering in the hundreds of millions per second, is known to human sense only as color. But there are many series of vibrations in this medium which the eye cannot count, just as there are sounds too high in pitch for the ear to hear. Those too slow are known as radiant heat; those too high 
are known as actinic rays, and can be perceived and counted only by the photographic plate.

There is another broad method of classification than this distinction between motion and stored motion. It is based upon the medium in which the energy finds expression, upon the particular kind or size of mass which happens to be in motion. Thus, we make a very easy and useful distinction between light and sound, though both are vibratory; or between the latent energy of gunpowder and that of a lifted tilt-hammer, though both be potential in form. Of the many different kinds of energy-forms thus arising the following discussion will make use of only the four (together with their distinctions between kinetic and potential) which are most commonly met with in the engineering of heat-engines, viz. :-

Mechanical: Kinetic and Potential;
Electrical:
Chemical :
Thermal :

Mechanical Energy is defined as that occurring in the motion or the stored motion, relatively to other similar bodies, of bodies of some appreciable size and mass. Such is the energy of weights, springs, bodies of water, etc.

Electrical Energy may be defined as that embodied in the relative motion or position of separation of the particles of some atomic medium, as yet not well defined or understood, but whose characteristics are so markedly distinctive from those of the other forms of energy that no more scientific definition is necessary here. The atoms embodying this sort of energy are known as electrons.

Chemical Energy is defined as that inherent in the relative stored motion or position of molecules of two or more 
different chemical natures; that is, such as that between a molecule of carbon and one of oxygen, of hydrochloric acid and iron, etc.

Thermal Energy is defined as that inherent in the molecules of a body by virtue of their motion and position relatively to other molecules of the same chemical nature within the same body.

Concrete illustrations of these several forms may be drawn from daily experience, viz. :-

Mechanical, kinetic: A hammer, a fly-wheel, or a projectile.

Mechanical, potential: A lifted weight, a distorted steel spring, or the water of a millpond.

Chemical, potential: The energy stored in fuel, in gunpowder, or in corrosive acids.

Electrical, kinetic: An electric current.

Electrical, potential: An electrical charge, in a Leyden jar or similar condenser.

Thermal, kinetic: The sensible heat or temperatureheat of a body, as of red-hot iron.

Thermal, potential: The latent heat involved in a change of physical state, as of the melting of ice into water or of the boiling of water into steam.

It is easily apparent that this list of the forms of energy would need to be expanded only to include magnetism, light, and sound to make it comprehend almost every possible phenomenon occurring on the face of the earth or in the surrounding universe. 
One cannot ponder upon this absolute constancy in amount of energy, underlying the most complex alterations in its external appearance, without being finally impressed with the fact that the alteration is merely external and that the real essence of the energy continues to exist, unchanged and uninterrupted; and that what we regard as a change does not occur in the thing observed, but merely in its locality and in its relation to the observer. For all of these forms of energy consist of motion or of stored motion; and both motion and position are purely relative.

Without any need for an attempt at building upon this fact a system of philosophy, it is nevertheless necessary that the engineer, of all persons, should realize this fact to his finger-tips. Otherwise he will frequently be deceived or caught napping. No pantomime actress was ever quicker or more capricious in her lightning-changes of costume than is energy. It is anything and anywhere. At one moment heat in the hundred-thousand-mile flames of the sun's surface, ninety millions of miles away; the next moment light in a man's eye; the next sealed in his brain as an optic impression and memory which he carries to his grave. Now the cold, black inertness of the coal-seam in the bowels of the earth; soon the glare of the boiler-furnace; then the invisible energy of the silent steam; a moment after, the battle of a mighty ship with the fury of the gale, or the peaceful glow of an electric arc miles away. But the actress remains the same. No matter what the guise, on the stage or in the world the impersonator retains the individuality of a continuous existence; and in the latter case the impersonator is always motion, - the motion or the stored motion of mass.

In illustration of this truth instances of such a change of costume, from every different one to each of the others, can 
easily be found, not only in the wide field of scientific experiment but in the much narrower one of the useful arts. They are all possible; they all occur in the everyday life of the universe; they are nearly all of them familiar to the average observer. Most of them are turned to account in the production of wealth. Thus:-

From Mechanical to Thermal :

The heating of a body subjected to friction or impact.

From Mechanical to Electrical :

The dynamo or the glass-plate electric machine. From Mechanical to Chemical :

The setting-off of a detonating compound, such as fulminate of mercury or nitroglycerine. ${ }^{1}$

From Electrical to Thermal :

The heating of any non-conductor by the passage of an electrical current.

1 This is one of the most obscure and unfamiliar of energy-transformations. The energy revealed in the decomposition of an explosive, which finds expression in heat, light, sound, and violent mechanical movement, is the equivalent of the chemical energy originally stored in the explosive; the phenomenon should be classed as a transformation of chemical into one or more of these other forms of energy. But every explosive, like a row of blocks stood upon end, needs some initial impulse or supply of energy from without in order to set in motion the process of energy-transformation. With the majority of them, as with gunpowder, this preliminary supply of energy must take the form of heat, in the process of ignition. But in many others, such as those mentioned in the list, heat will not initiate the explosion; a sudden jar will alone suffice. In this sudden shock to the explosive a minute quantity of mechanical energy is absorbed in upsetting the equilibrium of structure of a few molecules; the consequent release of energy from the fall and crash of these few into their more stable components upsets the rest, and the explosion ensues. This preliminary microscopic transformation of mechanical energy into chemical energy is the only instance of its class which has yet occurred to the writer ; but it is of considerable importance in the arts, for the detonation of the ordinary rifle-cartridge and of most heavy explosives depends upon it. 


\section{From Electrical to Chemical:}

The electrolysis of a chemical compound by the passage of current, as in electroplating, or in the charging of a storage-battery.

From Electrical to Mechanical:

The electric motor; the solenoid.

From Chemical to Mechanical:

The evolution of a gas from the combination of two solids or liquids. ${ }^{1}$ The chemical fireengine. Animal activity.

From Chemical to Electrical :

The evolution of an electrical current from a primary or secondary battery.

From Chemical to Thermal:

The combustion of fuel. Animal heat.

From Thermal to Mechanical:

The expansion of a heated body; the steamengine.

From Thermal to Electrical: The electropile. ${ }^{2}$

From Thermal to Chemical:

The lime-kiln; the growth of vegetation.

Not only the material arts, but the entire daily life-experience of man as well, swarms with a myriad of such

1 This transformation is a very common but, outside of the processes of animal life, not a very obvious one. The mechanical work performed by the cold evolution of gas from a combination of solids or liquids is usually only that of displacing the atmosphere. It is utilized in the arts, however, in the operation of chemical fire-engines and extinguishers.

2 The electropile is a true representative of this class of transformations, but one very narrowly limited in its applications. The invention of an apparatus which will perform this transformation upon a large scale, without the intermediate transformation into heat, as through the medium of a furnace, boiler, steam-engine, and dynamo, is one of the unsolved problems of the day which attracts inventors to its solution by the most unlimited promise of pecuniary reward. 
phenomena, all of which consist of and reveal transformations of energy from one outward aspect to another. With energy not only is frequent alteration of form possible, it is apparently inevitable. Not only do all material phenomena, but life itself - anything, in fact, which can be defined as an event or occurrence-consists of a series of energytransformations following one another in ceaseless continuity. There is, so far as the human mind can grasp, no possibility of a stop. With many forms of energy the transformations follow one another with a rapidity which the mind is entirely unable to comprehend; with others, such as those visible in moving machines, they are more deliberate and are easily traced; with others, such as those occurring in the heavenly bodies, the changes are so slow or infrequent that human experience is too brief to cover and perceive them directly, though their occurrence is known by scientific deduction.

Without doubt, light is one of the most evanescent of all forms of energy. Its natural speed of 186,000 miles per second carries it across all terrestrial distances in the twinkling of an eye, and it cannot meet an, opaque body without being transformed into heat. Yet the light travelling from the sun to the earth exists unchanged for over eight minutes; that coming from Sirius remains unchanged for some twenty years; while astronomers tell us that there are fixed stars, or suns, so distant that their light which we perceive to-day must have left its source before the birth of Christ and therefore must have existed as light for some two thousand years without alteration. Sound, too, is short-lived. Though much slower than light in transmission, the resistance which it meets in its denser medium, air, is so great that only a few minutes may elapse before it too becomes heat. Electricity is quite as shortlived as light. 
The other forms of energy change less frequently. Familiar types show the opposite extreme and apparently never change. The potential energy stored in the "everlasting hills" appears to be eternal; but the hills are but the creatures and toys of a childish ocean not yet fullgrown, which will yet be the death of them with its cloudbursts and tidal waves. The aged ocean is itself but the first-born of Mother Earth; she herself is but the baby of the solar system; and even the sun, patriarch and ruler of all the planets, sure to outlive in his enormous might the biggest of them, is having his age estimated by modern astronomers as by his teeth and the probable date of his death foretold in serious discussion. There is nothing known which is stable. There is nothing which will not, eventually, change into something else.

To the untrained observer this endless procession of alterations of form is as a kaleidoscope to the childish eye. All is bewildering confusion, - wonderfully beautiful, but distracting and leading nowhere. But to one who knows that the kaleidoscope consists of nothing more than a few bits of colored glass and three mirrors, its explanation is easy and the mystery is gone. The different bits can be identified and utilized to produce new effects at will. So with this ceaseless chain of energy-transformations visible in nature; we must first learn to identify the component parts, the several forms of energy. We watch a bit of sun-heat grow a tree; we fell the tree for fuel; fed into a boiler furnace it makes steam; with the help of an engine the steam makes motion, which drives a dynamo, etc. At each link of the chain the form of energy must first be identified. It then becomes clear that only the form, and not the quantity, of the energy has changed. In each case the senses are so deceived by the complete contrast between former and latter appearance, and by 
the apparently complete lack of continuity underlying the transition from one to the other, that the mind is with difficulty persuaded that there has been no change in reality and that the transformation has been, to that extent, a deception. Nevertheless, the unalterable fact is as has just been stated. The existence of the energy has been continuous; its locality and its effect upon us are what have changed. Its amount, its power, its possibilities, are just what they were before. And in each alteration of form the direction of the step shows no evidence of chance, of indecision, of mystery. Each is guided by absolute law, - by a law which can be identified, defined, utilized.

In order to trace this law, attention must next be turned to the question of the dimensions of energy.

The Dimensions of Energy. - If each of these diverse forms of energy be examined more closely, it will be perceived that it does not assert itself to our senses as a single simple thing, to be comprehended, as to amount and characteristics, at a single effort. Indeed, with all except one or two it will appear that neither our senses nor any instrument which we can construct will be affected by and measure its quantity directly. Thus, in its mechanical form, energy appeals to us, if potential, merely as a force. We cannot directly perceive what amount of motion has been stored therein or through what distance that force will act if released, though we may reason it out. So knowledge as to the force alone is no knowledge as to the amount of energy present. If the energy be kinetic mechanical in form, we usually can perceive it as a velocity. But the mass concerned is not directly visible, and the velocity alone will not measure the energy present. It is true that the ballistic pendulum will measure kinetic energy directly, and so will the electric watt-meter. But in each case the instrument takes cognizance of two factors going 
to make up the energy and integrates them accordingly. In reality it does within its own mechanism just what must be done in the measurement of the quantity of any and every form of energy: (I) The measurement of two distinct factors, and (2) their mathematical combination into a result which is a measure of the quantity of energy. In the most familiar case, that of mechanical energy, these two factors are force and distance, and in every other form of energy the two factors are these same familiar ones more or less disguised. A list of them is appended.

\begin{tabular}{|c|c|c|c|c|}
\hline \multirow{2}{*}{ Form of ENERGy } & \multicolumn{2}{|c|}{ FACTOR OF INTENSITY } & \multicolumn{2}{|c|}{ FACTOR OF EXTENT } \\
\hline & Name & Unit of Measurement & Name & Unit \\
\hline Mechanical, pot. & Distance. & Feet. & Force. & Pounds. \\
\hline Mechanical, kin. & Velocity. & Feet-per-second. & Mass. & Pounds $\div \mathrm{G}$ \\
\hline Electrical, pot. & Potential. & Volts. & Charge. & Coulombs. \\
\hline Electrical, kin. & Potential. & Volts. & Current. & Amperes. \\
\hline Chemical, pot. & Affinity. & - & Mass. & $\begin{array}{l}\text { Molecular } \\
\text { weight. }\end{array}$ \\
\hline Thermal, kin. & Temperature. & $\begin{array}{l}\text { Degrees (Fahr.) } \\
\text { absolute. }\end{array}$ & Entropy. & 一 \\
\hline Thermal, pot. & Temperature. & $\begin{array}{l}\text { Degrees (Fahr.) } \\
\text { absolute. }\end{array}$ & Entropy. & - \\
\hline
\end{tabular}

In this table the factors for the first five forms of energy are familiar to every college student. The factors, or dimensions, of thermal energy, however, do not seem to be a part of one's everyday experience, nor to be readily comprehended as factors of energy. It is for this reason that they are placed last in the list.

The fundamental idea of energy is based upon the combination of force and distance. In mechanical potential energy, where the idea originated, the two factors are 
plainly visible. What, for instance, is there about the factors of kinetic mechanical energy which connects them with those of potential mechanical energy, into which it transforms itself with the greatest readiness whenever opportunity offers?

The amount of energy resident in a moving mass is known to be expressed by the formula $\frac{1}{2} M v^{2}$, where $M$ is the mass and $v$ the velocity. But a moving mass is known to possess energy only because it will always overcome resistance in coming to rest. If it be brought to rest by means of a constant negative acceleration in the unit of time, one second, it will have traversed the distance $\frac{1}{2} v$. If its total energy is to be absorbed in this distance, it must be done by a resistance having a mean value of $\frac{1}{2} M v^{2} \div \frac{1}{2} v=M v$. But $M v$ is the force which, if applied constantly through the unit of distance to the mass $M$, will produce the velocity $v$, and hence will, if applied negatively, destroy the velocity $v$ within the distance $\frac{1}{2} v$. Thus kinetic energy is also a function of force and distance.

In the case of chemical energy the factors of force and distance, though not directly visible, are easily comprehensible. The molecules of certain chemicals possess a mutual attraction for the molecules of other chemicals entirely aside from the mutual gravitation of their masses. This attraction is known as chemical affinity. Thus, carbon and oxygen exercise a powerful attraction the one for the other. Hydrogen and oxygen do likewise; but in the latter case the affinity between the two is much more powerful than with carbon and oxygen. When either hydrogen or carbon burns, developing heat, the process is nothing more mysterious than a falling-toward-one-another of each pair of molecules, and the energy developed is exactly analogous to that developed when a weight and the earth, mutually attracting one another, fall together. 
Similarly, when steam is dissociated into its components, hydrogen and oxygen, or carbonic acid into carbon and oxygen, the molecules require to be separated against their mutual attraction. The process is exactly analogous to the separation of a weight from the earth against the mutual attraction, and the energy absorbed in the dissociation is entirely similar to that absorbed in raising the weight. In the infinite variety of combinations and dissociations of chemicals there are as many different degrees of affinity and of stages of more or less complete combination or separation as there are of different weights and levels in the field of mechanical potential energy.

The cases of electrical and thermal energy are not so simple in explanation as the above. Each consists of a series of vibrations, in its kinetic form, and of permanent separation in its potential form. In the case of heat these vibrations and separations concern the molecules of the body itself, or portions of them, in terms of their mutual cohesion; in the case of electricity they concern, supposedly, the electric atoms or electrons. Of the molecular construction of matter very little is yet known, so that it were unsafe to attempt a too close and accurate description of the ultimate natures of these phenomena.

But of this much we can be absolutely sure: If all matter, including the electrical, does possess component molecules, if these molecules be separated from one another by a distance considerable in proportion to their diameter, and if they exercise a mutual attraction for one another, then, in their vibrations within this attraction they must involve alternations of kinetic and potential energy, and are to this extent a dynamic parallel to a pendulum or to waves in motion upon the surface of a sheet of water, requiring a supply of energy from without to be set in motion, capable of transmitting that energy with great ease 
and little loss, and necessarily giving that energy out again before rest can again be attained. Similarly, in their static separation from one another against their mutual attraction they must involve a static parallel to a weight and the earth, requiring a supply of energy, or motion, from without for the performance of their separation, giving little evidence of the possession of the latent energy so long as the separation remains unchanged, and re-developing that same energy into visibility when again allowed to come together.

What is more essentially true than that kinetic energy is a disguised function of force and distance, is that potential mechanical energy is a disguised function of mass and velocity. It will be noted that all of the other items in the column of Factors of Extent of which anything definite can be said are mere measures of mass. Now mass, when the source of the idea is once analyzed, appears plainly as that portion or quality of the substance of the universe which remains constant. Indeed, it alone is that substance. All other features, such as weight, etc., are mere attributes, due entirely to the position of the mass in question relatively to other portions of mass.

The first law of physics, that of the conservation of matter, states this fact:-

"The mass of the universe remains unchanged and is unchangeable."

The definition of mass is the mere obverse of this :-

"Mass is the unchangeable in matter."

But the first law of energetics, that of the conservation of energy, states that the energy of the universe is unchangeable. Since all forms of energy are reducible to the motion or the stored motion of mass, and since the latter factor has just been defined as a constant, it follows that the former factor must also be a constant, when the total quantity in the universe is viewed as a unit. 
The truth of this statement will appear more clearly from further consideration of the question. For the present it is sufficient to note that, of the two factors going to make up each form of energy, one, of the list which the writer prefers to call the "factors of extent," is always a function of mass. It, in fact, measures the amount of the universe which is involved in the energy in question. The other dimension, of the list which he calls the "factors of intensity," is always a function of velocity of motion or of remoteness of separation of the mass involved. It is a function of the mutual relations existing between any two or more portions of the mass involved in the first factor.

It is quite consistent with a conception of the universe as a system of portions of mass involving various degrees of relative motion and position, that the several masses should be regarded as always remaining constant, from instant to instant, and that their integrated sum be also constant. It is also consistent with such a conception that the instantaneous relative motion or position of the individual component masses be ever varying; indeed, the very conception of motion implies it. And yet, if the centre of mass of the universe be imagined as fixed, or, which is the same thing, if these various motions and positions be regarded as purely relative, then the integration of their relative velocities and of their degrees of separation must always together amount to a constant.

If, then, to return to the list of energy-factors, the factors of the first column, those of intensity, be remembered as functions of relative velocity or of relative separation, it can be stated that in individual cases they will be ever found varying and variable, but in their summation or average they will always be found constant in quantity.

The three basic laws of energetics, approached in this way, may be stated as follows :- 
I. The Conservation of Matter: The mass of the universe ever remains constant in quantity.

II. The Conservation of Motion : The total intensity or potential of the universe ever remains constant in quantity.

III. The Conservation of Energy : The total energy of the universe, the product of the two foregoing factors, ever remains constant in quantity.

It has already been noted that there exists throughout the universe a tendency for every form of energy to transform itself into some other, and that although this tendency is in some cases resisted for periods of time seemingly very long, when compared to human standards, yet it always finally occurs. It is natural to expect that these relative motions and positions of the myriad of portions of mass which constitutes the known universe should be continually altering themselves into other motions and positions. It would seem that there must be some laws controlling these transformations, now permitting some transformations to take place with instantaneous rapidity and now delaying others for ages.

In the tabular list of page 20 the classification into factors of intensity and factors of extent was not made primarily upon the basis of the broad theoretic postulates enunciated above. It had been observed that the factors of energy possess two distinct sets of characteristics. Upon these characteristics the classification was based. The names intensity and extent were chosen as best representing those characteristics. ${ }^{1}$

${ }^{1} \mathrm{Mr}$. George Richmond, in treating of thermal phenomena only, makes use of the terms height and breadth instead of intensity and extent respectively, and there are many reasons why, especially in connection with graphic representation of energies by coördinate diagrams, his choice is to be pre- 
In all forms of energy it is the prime characteristic of the factor of intensity that it ever and always tends to a decrease and disappearance, by the fall of the energy of which it is a part to and through lower and lower degrees of intensity, until ultimately the zero of intensity might be reached. Thus, taking the most familiar form of energyfall first, mechanical potential energy always tends to decrease its factor of intensity, distance of separation, to lower and lower degrees of separation until there be no separation left. Water, attracted by the earth's gravitation and separated from it by evaporation into a rain-cloud, tends to fall nearer and nearer to the most intimate possible association with the earth, from rain-cloud to hilltop, from hilltop into mill-pond, thence to the tail-race, and from the tail-race to the sea; and if there were a passage from the sea to the centre of the earth, it would flow thither. Similarly, a steel spring, stretched by previous distortion to a distance of separation from its condition of equilibrium, tends always to return, through various intermediate degrees of separation, to the position of the closest possible approach to equilibrium.

In a like manner the mechanical kinetic energy of a moving body tends always toward a reduction in velocity, whether through the medium of friction, of impact, or of the imparting of a portion of its energy to other bodies in the form of motion.

Electrical energy, whether kinetic or potential, always tends to flow from the point of higher voltage to the point of lower, reducing its own potential as it goes.

In chemical energy the tendency exhibited in reactions and combinations is always away from the compounds of maximum chemical affinity and minimum chemical stability

ferred. The writer's reasons for preferring the terms he has adopted are for the sake of breadth of definition. 
to those of less and less intense affinity for one another until a level is reached where no further combination is possible.

In thermal energy is exhibited one of the most familiar illustrations of all, the constant tendency of heat to flow from the body or locality of higher temperature to that of a lower temperature. But this illustration does not fully cover the law. Heat tends strongly to alter itself to a lower temperature even when there is no opportunity for flow out of the body in which it lies into another colder one. Even if the body be completely isolated from the rest of the universe, by an insulation so perfect that both radiation and conduction are absolutely impossible, its heat will alter itself to a lower temperature by other processes when opportunity occurs. Discussion of the nature of this opportunity must be deferred until later in the argument.

The converse of this law is also true, viz.: That energy never will alter itself spontaneously and without transformation from a lower to a higher degree of intensity. Water will not flow uphill, nor any weight rise from the earth, nor a spring distort itself. A moving mass will not spontaneously increase its velocity, nor impart its energy to a mass moving at a higher velocity. Electricity will not flow against a superior potential, nor will a charge spark from one jar to another more highly charged. Chemical compounds will not combine, unaided, into compounds having a higher affinity than the originals. Heat will never flow spontaneously from a cold body to a hot one.

This being true, how is it that there ever exist any higher degrees of intensity, to maintain by their fall the current phenomena of the universe from which this law has been observed? It is unimaginable that they should all have come from an original store and that the universe 
is engaged in slowly "running down." It is obvious that all of these at first sight unattainable degrees of intensity in energy must have been somehow attained, and that by means of processes which must be as current and as natural now as at any previous time.

There are two methods by which rise of intensity is accomplished, and only two, and of these the first-mentioned is only local and temporary in its effect :-

(I) There comes from some outside source an additional supply of energy to force the apparently unnatural process; or

(2) There occurs an intermediate energy-transformation into some other form and back again.

Indeed, it is only so long as the energy remains in its original form that the spontaneous tendency to drop in intensity is manifested. Thus, water will not flow uphill; but it can be pumped up with the aid of some external supply of power, or, its potential energy transformed into kinetic and back again through the medium of the hydraulic ram, it will pump a portion of itself to a higher level than the supply. The kinetic energy of a moving mass cannot of itself take on a higher velocity; but enforced by an additional supply of energy from without it will take on a higher velocity, it will become a portion of a larger total quantity of kinetic energy having a higher degree of intensity; or, if inherent in an elastic body and meeting a body having velocity in the opposite direction, it will, by conversion into potential energy of distortion and back again, convert a portion of itself into a higher velocity. Electricity will not spontaneously flow to a higher voltage; but its voltage may be increased by being electrically reënforced; or it will raise its own voltage by means of a double transformation through a converter. Similarly, heat will not voluntarily alter itself 
from a colder to a hotter temperature; but it may be forced to do so by an external source of power acting through a refrigerating-machine; or it may be transformed into heat of any degree of temperature by means of a double transformation into some other form of energy, as into electricity, and then back again by way of the electric arc.

It is especially to be noted, in considering this question as to how the at first sight apparently unattainable degrees of intensity originated, that in the preceding illustrations reliance was placed upon the two methods of increase of intensity, viz.: -

(I) The simple addition of energy from some other portion of the universe;

(2) The transformation, into the form under discussion, of some other form of energy already existent.

It next appears that whenever the latter method prevails, whenever such an energy-transformation occurs, there appears to be no broad natural limit to the degree of intensity of the secondary form of energy. Thus, there is no broad law connecting the muzzle-velocity of a projectile with the chemical affinity of the gunpowder. There is a relation between the two, but it depends upon the relative mass of powder and ball; the relative diameter and length of gunbarrel, etc., etc. These are all accidental circumstances, not broad principles. The attainment of the highest muzzle-velocities has not resulted from the discovery of explosives of extreme chemical affinity. Similarly, in the electric arc, the temperature attained is not a basic function of the voltage producing the arc. Very high voltages apparently produce no higher temperatures than quite low ones may do, under favorable conditions. In any such case the intensity of the secondary form of energy appears 
to depend upon circumstances which may be called accidental in their character, and not at all upon the intensity of the primary form. It is plain that the phenomenon is such as to permit the attainment, in the secondary form of energy, of the highest degrees of intensity known to man, and that from comparatively low degrees of intensity in the primary form.

Upon further inspection it appears that these two processes by means of which higher degrees of intensity are attained are in every way the obverse of those by which intensity lowers itself. For when the energy of a body obeys the familiar tendency to fall in intensity, its quantity must always decrease. But, since annihilation of energy is impossible, decrease is possible only in one of two ways :-

(I) Dissipation, or the distribution of energy to other masses of similar character;

(2) Conversion into other forms of energy, or the distribution of energy to other masses of dissimilar character;

and these two methods are plainly merely the obverse of the two which are listed on the preceding page. Thus, the energy of a moving mass of appreciable size, such as that of a projectile, always exhibits a tendency to decrease its intensity, or in other words its velocity, of motion. It will always do this in one of two ways:-

(a) By meeting other bodies which are perfectly elastic and which will partake of the motion of the original mass, the result being a greater total mass in motion of a lower average intensity; or $(b)$ by meeting other bodies which are more or less inelastic, in which case friction and impact occur; and friction and impact are merely names for the conversion of mechanical energy into heat. 
Similarly, chemical energy will always, when permitted to free itself, either dissociate a larger mass than its own to a lower degree of chemical affinity, or it will convert itself into heat, light, electricity, sound, etc., which are merely the motions or positions of masses dissimilar to chemical mass.

Electrical energy will always fall in potential when permitted, and in so doing will convert itself into either heat, light, sound, chemical energy, or mechanical motion ; or, if in static form and free to conduct itself to a larger mass of surface, it will do so, but with a corresponding reduction in potential.

Heat, in the same way, will always tend to alter itself to a lower intensity, and in doing so either other masses of matter will be heated to temperatures lower than the original or new forms of energy, such as chemical dissociation or mechanical motion, will be produced.

If attention then be turned to the secondary bodies which are affected by these processes, it will be plain that they are undergoing, in each case, the obverse of the processes under discussion. That is, the intensity of their energy is being raised by one of two processes :-

(a) By the simple addition of energy from the primary mass ;

(b) By the conversion of the primary form of energy into their own.

These three statements of the two processes, on pages 29 , 30, and $3 \mathrm{I}$, show them to be identical; on each page they were approached from a different point of view.

Finally, if it be remembered that all forms of energy consist of the motion or the stored motion of masses of one sort or size or another, it becomes plain that even the conversion of energy into other forms is merely a transfer 
of motion from one mass to another, but in this case to a mass of a different kind. Thus, when the energy of a moving mass of appreciable size is converted into heat by friction, the parallel simultaneous motion of the molecules forming the body is altered either into other sorts of motion of those same molecules, in so far as the moving body itself is heated, or into varied motions of the molecules of the bodies with which it comes in contact. Similarly, the production of electricity or chemical dissociation from mechanical energy is merely the transfer of the paralle] motions of the particles of the original body into various peculiar motions or separations of the numerous masses of electrical or chemical atoms of the bodies affected. These bodies may remain stationary in relation to the surrounding universe, but they are in possession of active internal motion; and the original source of energy has been brought to visible rest.

\section{Summary}

The universe, viewed in this light, reduces to the following simple statement, which includes all known phenomena, whether simple or complex, whether inanimate or animate, from the motions of the heavenly bodies to the action of dust floating in the sunlight, from the quiescence of the clod to the most intricate and delicate processes of human life and thought, viz.:-

An infinite series of portions of mass, of infinite variety of size and form, linked together by mutual gravitational attraction, each associated with the others in a certain degree of separation and possessing a certain amount of motion. Both separation and motion are purely relative. Motion cannot exist without accomplishing separation, positive or negative. Separation cannot exist, appear, or disappear, without accomplishing motion, positive or nega- 
tive. Motion, therefore, cannot exist without causing change in both separation and motion. The same is true of separation.

Newton's "First Law of Motion" is in no conflict with the above statement, because the former, in its application to a given mass, assumes the non-existence of all other mass. The law stated in the preceding paragraph assumes in its premises the existence of all other mass, - not merely a few portions of it, but an infinity of portions, extending to unthinkable limits.

Motion and separation are purely relative and are interchangeable. They together constitute the intensity of the energy of the universe. The mass embodying the motion or separation constitutes the extent of the energy of the universe. Both intensity and extent ever remain constant in quantity.

The intensity of the energy of a given mass always tends to decrease. When that energy consists of separation, the separation tends to disappear, producing motion; when it consists of motion, the motion tends to disappear, producing separation.

When the motion is transferred from one portion of mass to another (which constitutes what is known as a "transformation" of energy), the proportion of intensity of motion or separation to extent of mass involved in the new form is independent of the degree of intensity in the old; the degree of intensity in the new is always a maximum controlled by environment.

The laws which govern the when and how of such conversions, and which dictate the limit which has been cloaked under the expression "the maximum permitted by environment," are of the most intricate. Their discovery and statement are the work of all the existing sciences and of all those yet to be constructed, in combination. 
These principles may be stated in more concrete and popular language in the following form.

The primary law of energetics is that of the

\section{Conservation of Energy}

"Energy is eternal. It can never be either destroyed or created; whenever one form of energy disappears it must reappear in another form in equal quantity."

This law comprises two minor principles :-

\section{The Conservation of Mass}

Matter is eternal. It can never be destroyed or created; only the mutual relation of its parts can be altered.

\section{The Conservation of Motion}

Motion is eternal. The total amount and the average intensity of the motion or the separation (the "stored motion") of the universe remains ever constant and can never be destroyed or created; it can be altered only in the mutual relations of its parts.

Mechanical energy and thermal energy, by virtue of their existence as energy, come within the above law as special illustrations. Hence the primary law of thermodynamics, the so-called

\section{First Law of Thermodynamics}

Heat can be neither destroyed nor created. When converted into motion or derived from motion, the energy disappearing is always the equivalent of that reappearing. ${ }^{1}$

The second great law of energetics is that of the

1 Of the two minor principles noted above only the first applies in the study of thermodynamics, and it is of passive rather than of active interest. The second, which covers all the motion of the universe, naturally cannot apply to the one peculiar portion of that motion which we call heat. Any one portion of the motion of the universe, such as heat-motion, may decrease or increase; it is the sum total only which remains cunstant. 


\section{INTERCHANGE OF ENERGY}

The form of energy is fickle and evanescent. Neither motion nor separation can exist in mass wirhout alteration, either into the other one of the pair or into the motion or separation of other portions of mass. This alteration always takes place from a condition or locality of higher to one of lower intensity, and never the reverse.

Thermal energy, in obedience to this generalization, presents the

\section{Second Law of Thermodynamics}

Heat is fickle and evanescent. It ever tends to alter itself from higher to lower temperatures whenever opportunity offers, either by dissipation or by conversion into other forms of energy. It will never spontaneously alter itself from a lower to a higher temperature, but may be forced to do so in either of two ways, viz.:-

(I) By a supply of energy from without;

(2) By a double intermediate transformation into some other form of energy and back again.

When the tendency of heat to fall in temperature was first noted and formulated into a law, it was also noted that its dissipation amongst larger and larger masses was the commonest method of temperature-fall. No return of the heat to higher temperatures seems to have been noticed, although it is occurring upon every hand. The situation was therefore covered by the so-called "law of the dissipation of energy,"' viz. :

The entropy of the world tends to a maximum and the temperature to a minimum.

The modern statement of both sides of the law, in similar language, would be:-

The total entropy and the average temperature of the universe remain constant. 
But no such law may probably be true, for entropy and temperature are respectively the mass and the intensity of only one sort of energy. Since there is as yet no evidence accumulated which reveals any fixity of proportion between the several sorts of energy in the universe, although in all probability that proportion is rigidly controlled by natural law, no law similar to the above may be stated which confines itself to a single form of energy, such as heat. 


\section{CHAPTER II}

\section{THE CYCLE}

THE tendency of energy to fall in intensity, whatever be the form of energy concerned, almost always results in the more or less complete transformation of the energy into some other form. But in each of these cases of transformation it is to be noted that the continuity of transformation is limited. Thus in the case of a weight suspended above the earth, it may continue to fall and to convert its energy into a kinetic form only until it reach the earth. That once reached its potential energy is, in one sense, all gone; its intensity of separation is simultaneously all gone, and the progress of conversion must cease. The matter which had recently served as a medium for the desired transformation of energy is no longer useful for that purpose.

This fact is a serious obstacle in the practical application of the process in the arts. A supply of energy must be continuous in order to be useful. Further, it is not ordinarily possible to look to a continuous, inexhaustible supply of matter containing the particular form of energy available for conversion. We must convert an indefinite amount of energy from the available to the desired form by means of a finite amount of matter, which must be utilized and relied upon as a carrier for the energy. The only known method by which such a carrier can perform continuous energytransformation is by endless repetition of the process. Thus, water endlessly repeats the process of fall from the rain-clouds to the mill-pond and thence to the sea, and of evaporation from the sea to the cloud again. 
The Working-substance. - The particular form of matter used as a carrier in any such repeated conversion is called the working-substance. The repeated process by which it effects a continuous conversion of energy is called a cycle. The latter may be defined as follows:-

A cycle is a series of processes, usually four in number, by the repetition of which a finite amount of matter may effect a continuous or infinite conversion of energy from one form to another.

It is in the application of thermodynamics to the design of heat-engines that the study of the form of cycle involved becomes of prime importance, much more so than in most other classes of energy-conversion. But as the conception of heat as a form of energy, and particularly as its two dimensions, temperature and entropy, are more difficult of comprehension than is the case with some more familiar and concrete forms of energy, the study of the thermodynamic cycle will be presented in the following pages by a running parallel between it and a similar conversion of potential mechanical energy, as of stored water-power, into some other form, and of heat-energy into some mechanical form.

In every such case the primary form of energy is composed of two factors, viz. : height and weight in the first case, temperature and entropy in the second. If the weight of a given mass be assumed to be constant over all ordinary variations in height, which is very nearly true, it would follow that the potential energy involved in the weight was equal to the height multiplied by the weight. In fact, in all hydraulic engineering we rely upon the formula that $E=H \times W$, where $E$ is the energy, $H$ the height, and $W$ the weight. But this formula is based upon the assumption that all changes of weight take place at a 
constant fixed height, and is therefore special in its character. To state a general equation based upon the same idea, recourse must be had to the calculus. The equation then becomes

$$
d E=H d W .
$$

This is the fundamental equation of potential mechanical energy.

In the case of heat-energy the situation is not quite so simple; at least it is not so apparent. Temperature has been defined as some function of the square of the velocity of translation of the molecules composing a mass of matter. But entropy has not yet been defined to the comprehension with even this degree of accuracy of conception. Its origin arose from the fact that the early founders of the science of thermodynamics found that in any alteration in the quantity of heat in a body the ratio $\frac{d Q}{T}$, wherein $Q$ is quantity of heat and $T$ the absolute temperature at which the alteration takes place, was a very frequently recurring and a convenient and useful function for a variety of calculations. They therefore gave it a name, entropy. The fundamental equation of all thermodynamic calculation therefore is

$$
d N=\frac{d Q}{T}
$$

where $N$ is entropy, $Q$ the quantity of heat involved in the change, and $T$ the absolute temperature at which the change occurs.

This equation gives the mathematical definition of entropy. Its definition in general terms is :-

Entropy is that quality of a body which increases whan heat is added to the body, which decreases only whon heat is abstracted, and consequently remains constant only when heat is neither added nor abstracted. 
Equation I easily converts itself into $d Q=T d N$, and in this equation we see great similarity to the equation connecting the two dimensions of potential mechanical energy, just given with water-power as an illustration. It is the integration of this equation, or

$$
Q=\int_{T_{1}}^{T_{2}} T d N
$$

which gave rise to Zeuner's name for entropy, heat-zweight; for in the energy developed by the fall of heat from the condition $T_{1} N_{1}$ to the condition $T_{2} N_{2}$, as given by the above equation, the entropy plays a very close parallel to the function of weight in the energy developed by the fall of a mass from a condition $H_{1} W_{1}$ to a condition $H_{2} W_{2}$.

These two definitions, the mathematical and the general, must suffice for the identification of entropy with mathematical accuracy.

But an approximate conception of its nature which is much more useful than the foregoing is to be had from the table of dimensions of energy given on page $2 \mathrm{I}$. It will become very clear, as the study of thermal phenomena proceeds, that entropy belongs in the column of factors of extent. When a thermic body is left thermally isolated, its entropy never alters in quantity, as its temperature may. When the body meets another and exchanges energy of like sort, there appears no law of connection between the direction of the interchange and the degree of entropy present. In all such ways entropy shows none of the characteristics whieh appear so plainly as the distinguishing characteristics of the factors of intensity and which form the basis of the Second Law of Energetics. Clearly entropy is a dimension of extent. ${ }^{1}$

${ }^{1} \mathrm{As}$ to the idea frequently promulgated at present in a manner far too dogmatic to harmonize with our meagre knowledge of the truth, viz.: that 
If so, entropy must be either mass itself or else some close function of mass. There are obstacles in the way of further definition of the first hypothesis; there is no basis which visibly directs one to a determination of the function referred to in the second hypothesis. So that further elaboration of the definition seems impossible. Yet the original statement remains unshaken.

It will develop, upon the most superficial inspection of thermal phenomena, that entropy is an active variable. If entropy be mass, the inevitable deduction must be, since the mass of a body remains constant during heating or cooling, that the proportion of the mass of the body which takes part in thermal vibration must be variable. Since thermal energy is normally distributed evenly throughout the body containing it, this must mean that there is a variable portion of the mass of each atom which enters into the motion which constitutes thermal energy. This variable portion, according to the first hypothesis given above, is the entropy of the body. If not, it is at least an active factor in that entropy. ${ }^{1}$

entropy is a mere mathematical ratio, heat divided by temperature, it is a brief task to point out its untenability. Heat is energy, an eternal, indestructible reality. Temperature is a mere dimension, quite destructible, of that reality. Their natures are totally incompatible. There is no more possibility of a ratio between heat and temperature being nothing more than a ratio than there is of a ratio between area and length being nothing else.

Nor does the hypothesis that temperature is itself energy (based solely upon assumptions which apply only to a single form of matter, the gaseous) help the situation. Both the isothermal and the constant-heat proccsses (see pp. 47 and 105) deny the tenability of the hypothesis. In the first, energy is absorbed with no increase in temperature; in the second, temperature disappears with no reappearance of temperature. Both processes show an irreconcilable conflict between the hypothesis and the First Law of Thermodynamics.

1 This hypothesis is in no way untenable in the face of the present aspect of molecular physics. The word atom has completely lost its pristine significance of something akin to a microscopic billiard-ball. The atom has never been anything more than a concept, based upon certain assumptions in order to 
Let the two pairs of factors which go to measure energyquantities of the potential-mechanical and the thermal types respectively be represented by ordinates and abscissæ of two respective sets of rectilinear coördinates, the former as in Fig. I and the latter as in Fig. 2. Let $O W$ be an axis upon which is measured weight, $O H$ an axis on which is measured height, $O T$ an axis on which is measured (absolute) temperature, and $O N$ an axis of entropy.

Let us suppose that the point $A$, in Fig. I, represents the condition of a mass having the height of separation from the earth of $H_{1}$ and the weight $W_{1}$; then its inherent potential-mechanical energy must be $E=H_{1} W_{1}$. Similarly, we may suppose that the point $A$ of Fig. 2 represents the thermal energy of a mass possessing an absolute temperature $T_{1}$ and an amount of entropy $N_{1}$; but we cannot similarly assume that its total thermal energy is $Q=T_{1} N_{1}$.

clarify certain obscure phenomena. These assumptions have had to grow in complexity with the growth of knowledge, until it is now accepted that what we attempt to define as an atom is merely a mass of matter very much smaller than human dimensions, but which, instead of being an indivisible, elementary sort of thing, may be and is probably as complex within itself as are the largest known masses of matter. So that it is quite feasible to suppose that varying portions of its mass may enter into those motions and positions which we lump under the title of thermal energy.

Moreover, it happens, rather unfortunately, that practically all of the brilliant investigations of the mathematical limits of molecular action have included in the premises the assumption that the mass involved was constant. The sole justification of this is the familiar fact that heating or cooling does not alter the total weight of a body.

For this reason, too, the hypothesis that entropy is merely mass has not progressed in limitation. It has neither been proved nor disproved. To the student who does not find that it aids his comprehension, complete progress through mathematical thermodynamics may be made by means of the two exact definitions of entropy already given; but it will be purely mnemonic in its character and correspondingly limited in usefulness. To the student who takes the trouble to study the real significance of thermodynamic phenomena this hypothesis that entropy is thermal mass, although not demonstrably exact, will be found to be a great ancl proper aid. 
For of mechanical energy it is known that it must always equal distance times force, but of thermal energy it is only true that $d Q=T d N$.

In Fig. I suppose that the weight at $A$ be released from its position. It will immediately fall toward the earth, or along the vertical straight line $A B$. Suppose that at $B$ further fall be impossible, by reason of some practical limitation. The weight, now being useless for the further development of power, must be gotten rid of. If it be water, it is easily poured away, as into a tail-race. In fact, the processes depicted in Fig. I are most clearly imaginable as being carried out by a water-wheel made up of an endless chain of buckets passing over four pulleys, one at each corner of the rectangle. Each increment of departure from the axis $O H$ should then be understood as involving a corresponding increase in weight, as of water poured into the buckets; each approach toward $O H$ should be taken to indicate a proportionate decrease in weight, as of water poured out of the buckets. In this sense the line $B C$ represents the emptying of the buckets at the tail-race level.

It may happen, from practical limitations, that when the point $C$ is reached no more weight can be disposed of. Either no more water will flow out; or $\mathrm{H}_{2} \mathrm{C}$ may be taken as measuring the weight of the empty buckets themselves. If so, this weight must be raised to the $H_{1}$-level before more water can be taken in for the performance of more work. So the weight $\mathrm{H}_{2} \mathrm{C}$ is lifted along the line $\mathrm{CD}$ to $D$.

The empty buckets can now be filled, at head-race level, along the line $D A$, after which the process may be repeated indefinitely.

In this cycle the fall of the weight $W_{1}$ along the path $A B$ develops the work $W_{1}\left(H_{1}-H_{2}\right)$. In other words, the secondary form of energy is abstracted, after formation at the expense of the primary. Along $B C$ no work is done. 
Instead, energy of the primary form is abstracted. Along $C D$ the negative work $W_{2}\left(H_{2}-H_{1}\right)$ is done upon the working-substance by some exterior source of power, or, in other words, the secondary form is supplied to the system, being at the same time transformed back into the primary form. Along $D A$ no work is done, positive or negative. Instead, the primary form is supplied. ( $D A$ is most naturally taken

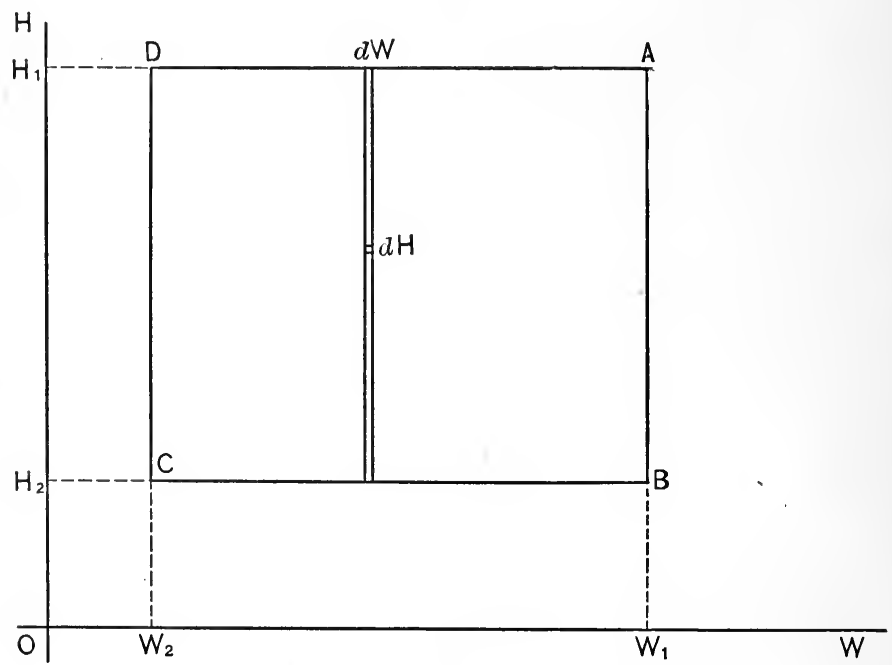

FIG. I

as the initial process of the four.) Therefore the total net work done by the working-substance must be the algebraic sum of the two quantities visible along $A B$ and $C D$, or

$$
\text { Work }=\left(W_{1}-W_{2}\right)\left(H_{1}-H_{2}\right) \text {. }
$$

But in this expression the first parenthesis is the width of the rectangle $D A B C$, and the second is the height. Therefore, in a rectangular cycle such as this the net quantity of energy transformed is measured by the area enclosed. 
It is evident that this same result could be reached by subtracting from the primary energy entering the system along $D A$ that rejected along $B C$. The difference, which disappears during the cycle, must, according to the First Law of Energetics, reappear in the secondary form.

If attention be turned from this cycle of finite dimensions to the infinitesimal rectangular cycle having sides of

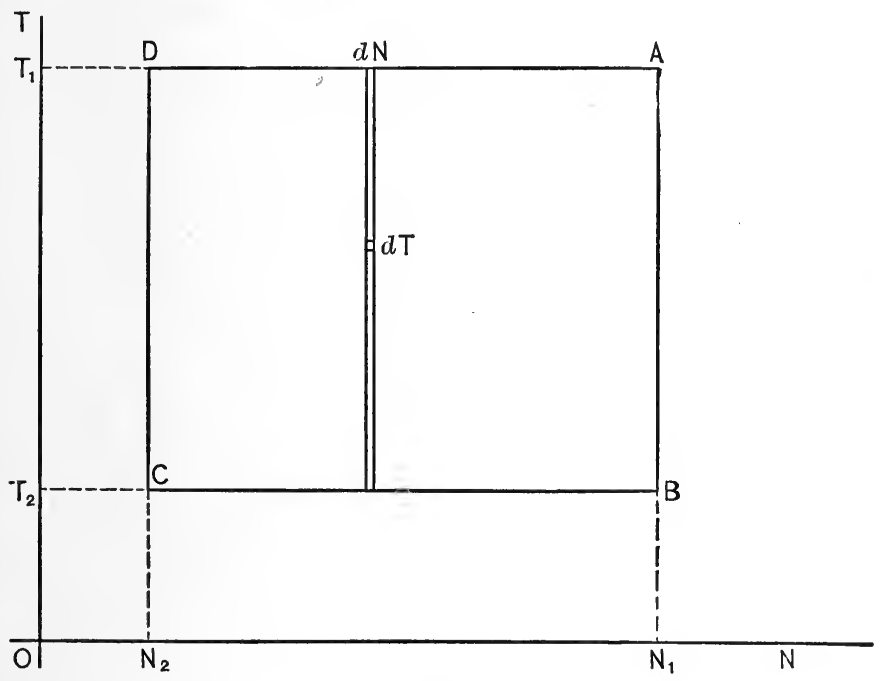

FIG. 2

lengths $d W$ and $d H$ respectively, shown in the centre of Fig. I, it is plain that this argument must equally apply; or, in other words, the work done in this infinitesimal cycle must be measured by its area, given by the expression

$$
d(d \text { Work })=d W d H \text {. }
$$

If this equation be true, the sum of any number of such equations must be true ; that is, the equation may be integrated. Such integration will result graphically, from the 
second member of the equation, in the development of a closed area, its form depending upon the particular functions existing between $W$ and $H$. It will result physically, from the first member of the equation, in the net total of energy transformed from the primary to the secondary form by the summation of rectangular infinitesimal cycles; and the physical significance of this summation of cycles, since their contiguous sides must always represent two identical processes in reverse directions and hence cancelling each other, is the same as that of a series of processes represented by the free sides.

It is plain that any closed area may be considered as the summation of such infinitesimal rectangular cycles. Hence the conclusion is drawn that -

If, in any series of height-weight processes forming a closed cycle (that is, one bringing the system back to its original position and weight) these processes be represented by lines forming a closed diagram, it must be true that the work done during the cycle will be given by the integration, to the proper limits, of the equation

$$
\text { Work }=\iint d W d H \text {. }
$$

But this integration will also result in a measure of the area enclosed by the diagram, from which may be drawn the proof that in any height-weight diagram the work done by a closed cycle is measured by the area enclosed by the figure depicting that cycle.

If attention be returned to Fig. 2, it will be obvious that the rectangle $A B C D$ represents no such familiar series of processes as does that of Fig. I. Nevertheless, it is just as true to life and just as frequent in occurrence; but it is much more obscure. If heat of the condition depicted at $A$ (Fig. 2) be given the opportunity, it will fall in tem- 
perature down the line $A B$ just as promptly and just as accurately as will water at $A$ (Fig. I) fall down the plumbline $A B$; and in falling it will just as surely develop work. At $B$ (Fig. 2) it may have reached the temperature of surrounding objects. If so, it will be prevented thereby from falling farther, at any rate usefully; just as the water-weight at $B$ had reached a point where it could fall no farther advantageously. It must therefore be rejected.

This is supposedly done along the line $B C$. The line $B C$ therefore indicates the abstraction of heat at constant temperature, just as in Fig. I it indicated the abstraction of weight-energy at constant level.

At $C$ it may be supposed that no more heat can be disposed of. If so, the rest must be lifted again, up the line $C D$, to the original temperature-level, by an expenditure of energy brought in from without. The heat-carrier, whatever it may be, is then ready to receive another charge of heat along the line $D A$ and to repeat the process indefinitely. This addition of heat necessarily implies increase in entropy. The increase is here supposed to take place at constant temperature.

In the case of the water-power of Fig. I, it is comparatively easy to follow with the imagination the several processes of taking in weight, letting it fall, emptying the residue, and returning the empty bucket. In the case of the heat-engine of Fig. 2, however, the processes are not so easy to follow. They are entirely unfamiliar, and it is only with the help of the water-wheel simile and the other analogies which have been presented that any useful comprehension of them may be had.

Isothermals. - The processes $D A$ and $B C$ are called isothermals, i.e. occurring at constant temperature. They necessarily involve, since the entropy increases or decreases, the addition or abstraction of heat. The most 
familiar instances of isothermal action are found in the melting of ice or the vaporization of water under constant pressure. In each case large quantities of heat enter or leave the body, yet the temperature remains unchanged.

Adiabatics. ${ }^{1}$ - The processes $A B$ and $C D$ are called adiabatics. Their location upon the diagram, the entropy remaining constant while the temperature alters, expresses graphically what the definition of the adiabatic does in words: An alteration in temperature without addition or abstraction of heat, or, in other words, while completely isolated from heat-exchange with the surrounding universe.

There lies herein a seeming paradox which must be cleared from the student's mind before further progress may be made, viz.: If heat be neither added to nor abstracted from the body, how can the temperature of the body, and also (as is clear from the diagram) its quantity of heat, be altered? The answer is: By conversion into work. During the adiabatic fall of heat no heat is lost as heat to the surrounding universe; but heat is steadily lost by conversion into the form of work. There is no heat abstracted; but there is heat lost, for work has been abstracted.

Similarly, during adiabatic rise of heat in temperature no heat is added to the body; but heat is gained, in the form of work, from the outside universe. In the adiabatic processes there is no exchange of heat between the body and its surroundings; there is an exchange of energy, however.

The process may be perfectly paralleled by a man who goes down town with his pockets full of money. He may not have been robbed of a cent, yet he may return with his pockets empty; but the supposition is that his arms are full of bundles which he has purchased. Not a cent has

1 The word adiabatic signifies "without exchange." 
been abstracted from him, yet he has ceased to possess his money; it has been transformed into another form of economic energy.

The adiabatic process may be said, in one very true sense, to be the only natural heat-process, for it is the only one in which the body acts freely and spontaneously of itself, without thermal influence from without. It is the process which every quantity of heat would follow if left to itself. Hence it is of prime importance in thermodynamic work as being the process from which all heat-processes depart only so far as they are forced to do so by purely external and often artificial forces. The adiabatic fall of temperature, which every portion of heat spontaneously undertakes when permitted to do so, is as natural a specimen of gravitation as that which called Newton's attention to the force which holds the universe together. The adiabatic is the plumb-line of heat-energy.

In this cycle of heat-processes the quantity of primary energy or heat supplied along the path $D A$ can be found by the integration of Equation 3. Since in this case $T$ is a constant, $T_{1}$, the integration becomes

$$
Q_{1}=T_{1}\left(N_{1}-N_{2}\right)
$$

Similarly, the quantity of heat extracted. along $B C$ becomes

$$
Q_{2}=T_{2}\left(N_{1}-N_{2}\right) \text {. }
$$

Subtracting, according to the First Law of Thermodynamics, the total quantity of secondary energy, or work, appearing as the result of the complete cycle must be

$$
Q_{w}=Q_{1}-Q_{2}=\left(T_{1}-T_{2}\right)\left(N_{1}-N_{2}\right) \text {. }
$$

For along $A B$ and $C D$ no primary energy is added to or abstracted from the system.

But in this expression the first parenthesis is the width of the rectangle $D A B C$ and the second is the height. 
Therefore, in a rectangular entropy-temperature cycle such as this, the work done is measured by the area enclosed.

From this idea can be developed the proof that -

In any closed entropy-temperature cycle the quantity of heat transformed into work is measured by the area enclosed, in a manner similar to that used with the height-weight cycle. In this case, however, it is important to establish one of the

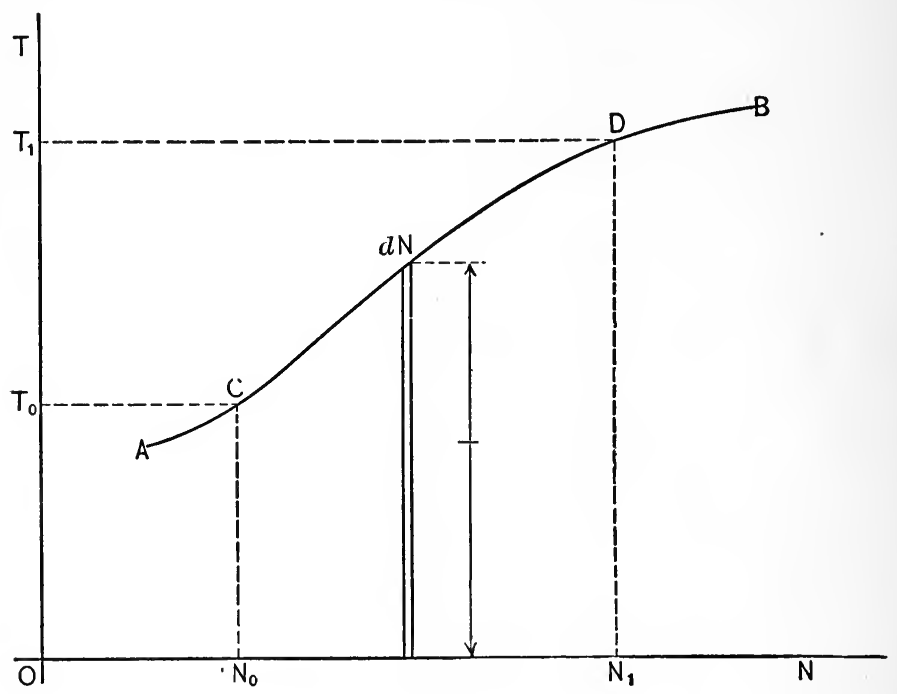

FIG. 3

intermediate steps as a general principle. This can be done by means of Fig. 3 .

In Fig. 3, let $A B$ be any heat-process whatever. Equation 2, transformed, gives

$$
d Q=T d N
$$

The right-hand member of this equation is represented geometrically by the elementary vertical strip having the 
width $d N$ and the height $T$. The integration of the above equation to any limits, such as $T_{0}$ and $T_{1}$ or $N_{0}$ and $N_{1}$, gives, algebraically, $Q$, the quantity of heat involved in the process, and geometrically, the area between the curve $A B$ and the zero-axis of temperature and between the limiting ordinates, or the area $N_{0} C D N_{1}$. Since $A B$ is any curve and the limits chosen were any limits, the following law may be deduced:

If any heat-process be depicted by a curve in an entropytemperature field of coördinates, the heat handled during the process is measured by the area between the curve, the zeroaxis of temperature, and the limiting ordinates.

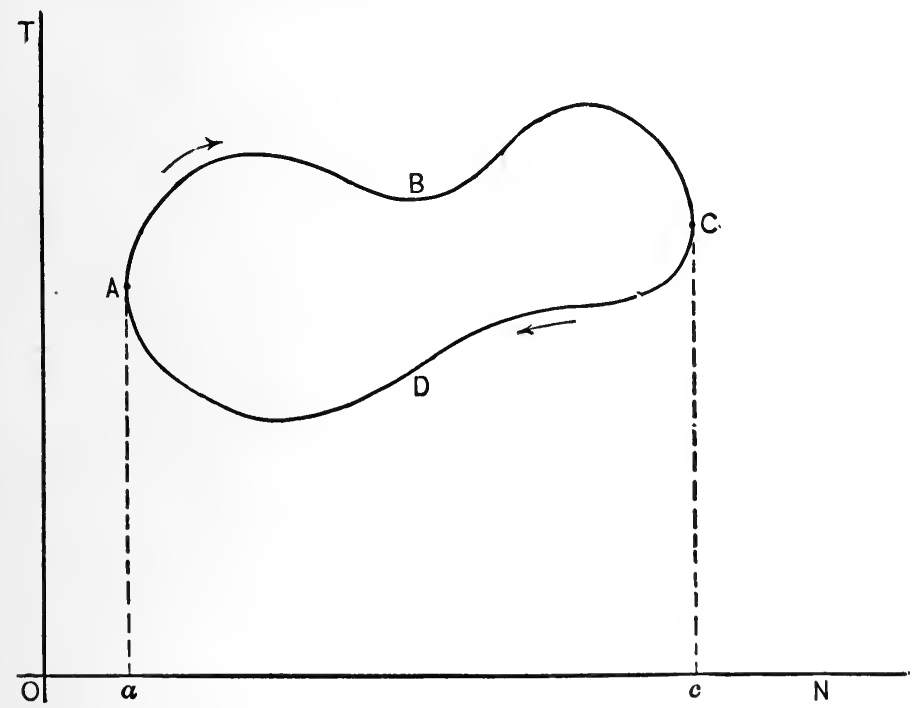

FIG. 4

If attention be next turned to any closed diagram on an entropy-temperature field of coördinates, such as that shown in Fig. 4, it is plain that it may be considered as made up of two portions, viz.:- 
(I) The portion $A B C$ in which the entropy changes from a minimum at $A$ to a maximum at $C$. This portion must indicate the addition of heat to the system. This quantity of heat supplied will hereafter be referred to by the symbol $Q_{1}$.

(2) The portion $C D A$ during which heat is abstracted from the system. The quantity of heat abstracted will be represented by the symbol $Q_{2}$.

By the preceding argument, $Q_{1}$ is measured by the area $a A B C c$. Similarly, $Q_{2}$ is measured by the area $c C D A a$. The heat converted into work must be their difference, or

$$
Q_{w}=Q_{1}-Q_{2}=\text { the area } A B C D .
$$

Since Fig. 4 represents any closed diagram, the argument is a general one.

Therefore, if any closed cycle of heat-processes be represented on the entropy-temperature ficld of coördinates by a closed figure, the heat converted into work at each revolution of the cycle is measured by the arca enclosed.

Conditions Essential to the Production of a Cycle. - The truth of the following statement of the conditions which must exist before a closed cycle such as that just discussed may come into existence will be obvious from the preceding argument. The original statement, made in reference to thermodynamic cycles only, was defined so long ago as I824, by Sadi Carnot, then a lieutenant in the French army. The modern, corresponding statement, which applies broadly to all forms of cycles, conducting transformations between any possible pairs of energy-forms, is the following:-

\section{The Third LAW of Energetics}

For the continuons, cyclical transformation of any primary into any secondary form of energy, within the con- 
fines of any limited portion of matter, four things are essential: -

I. A source of supply of the primary form;

II. An absorbent of the primary form;

III. A positive difference of intensity between the two;

IV. An absorbent of the secondary form.

(In the third condition the word "positive" is understood to apply when the intensity of II is subtracted from the intensity of I.)

As with the other general laws of energetics, when the two forms of energy concerned are heat and work respectively, this general law becomes one of the special laws of thermodynamics, viz. :-

\section{The Third Law of Thermodynamics}

For the continuous, cyclical transformation of heat into work, within the confines of any limited portion of matter, four things are essential: -

I. A source of heat;

II. An absorbent of heat, or "refrigerator";

III. A positive difference of temperature between the two;

IV. An absorbent of work.

To illustrate from natural facts, taking first the simplest and most familiar form of useful cycle, that between the potential mechanical energy of water-power stored in a mill-pond and the kinetic mechanical energy of moving machinery, it is obvious that the four essentials here are:-

I. The mill-pond;

II. The tail-race;

III. A difference of level, or "head," between the two;

IV. The driven water-wheel. 
Remove from the list any one of the four and it is obvious that the whole process must come more or less promptly to a stop.

Similarly, in thermodynamic work, the four essentials to the conversion of heat into work are already familiar to the student, although their exact identity and relations have probably never been pointed out to him. Taking the steam-boiler and steam-engine as an example, they are :-

I. The furnace;

II. The "refrigerator," or condenser, which may be the atmosphere or may be a current of cold water ;

III. A difference of temperature between the two;

IV. The steam-engine piston and the resisting machinery which is attached to it. ${ }^{1}$

1 It is of further and much wider interest and value to call attention to the application of the same law to transformations between other pairs of energyforms from the list given on page 20. The study of electrical engineering gives rise to a large number of cycles between electrical energy upon one hand and thermal, chemical, or radiant energy on the other, in each of which the four essentials may be traced. The study of chemistry, and particularly of organic chemistry, is productive of a much greater number of cycles; for there occur a much larger number of chemical compounds of different degrees of affinity than the number of different degrees of electrical potential which are commonly in use in the arts. Of all such instances by far the most interesting and in one way the most simple, although in other ways it is the most complex, is the cycle between animal and vegetable life. The broadest chemical basis upon which it rests is that of the oxidation of carbon and hydrogen, with the obverse dissociation. It also rests broadly upon the chemistry of the nitrates, and a myriad of other chemical reactions enter into the complete scheme; but none of them could go on without the oxidation which is the basis of the whole.

In this broad cycle it is the office of vegetable life to produce intensity of chemical affinity by the dissociation of the carbonic acid of the air into carbon and oxygen and of the water of the earth into hydrogen and oxygen, by the aid of sun-heat acting through the medium of the chlorophyl of the leaves. The oxygen is set free to the atmosphere and the carbon and hydrogen are stored in the plant in the form of various hydrocarbon compounds. 
The Reversed Cycle. - If attention be returned to the rectangular cycles of Figs. I and 2, it will be clear that nothing in the discussion of them has limited their dimensions. Vertically or horizontally, their limits may be separated by as great or as small a distance as accidental circumstances may dictate. This being so, let it be imagined that the vertical sides of the rectangle be mutually approached. Carried to the limit this process will result in their coincidence. If the displacement of the sides be still further continued, it must result in the rising side being to the right of the falling side. In order that the horizontal processes may close the cycle they will have to be reversed in direction; in other words, the direction of rotation of the entire cycle will have altered from a clockwise to a counter-clockwise one, as in Fig. 5. This means that at the upper level what was formerly the source of energy has become an absorbent; what was originally the absorbent has become a source of supply.

If computation of the areas measuring the energy-quan-

It is the office of animal life to produce intensity of mechanical energy and of animal heat, to the destruction of the intensity of chemical affinity just referred to, by combining within its body the hydrogen and carbon taken in as food with the oxygen of the atmosphere. At the same time, carbonic acid is rejected into and is stored up by the atmosphere.

Thus the two forms of life work together, to the destruction by each of the form of energy which it consumes and to the production of the form which the other consumes. Neither, so far as we know, could exist without the other. By the balance between the two the equilibrium of earthly conditions is maintained. Energy, intensity, and life are alike conserved.

It is not the purpose of this work to develop farther the study of such cycles. It is all-important, however, that the student should see clearly that the laws which are under discussion in the present chapter are not narrow ones, applying only to certain mechanical devices, but are broad principles which cannot be broken nor varied by any accident of form or locality whatever. In this broad sense, heat-engines are like all other portions of the universe and all forms of life or activity are like heat-engines. 
tities be made anew, it quickly becomes evident that the energy supplied at the lower level is less than that rejected at the upper level by the area of the cycle. According to the First Law, that of the Conservation of Energy, the difference must be supplied from without. A comparison of the energy transformed on the rising side of the cycle with that reversely transformed on the falling side shows

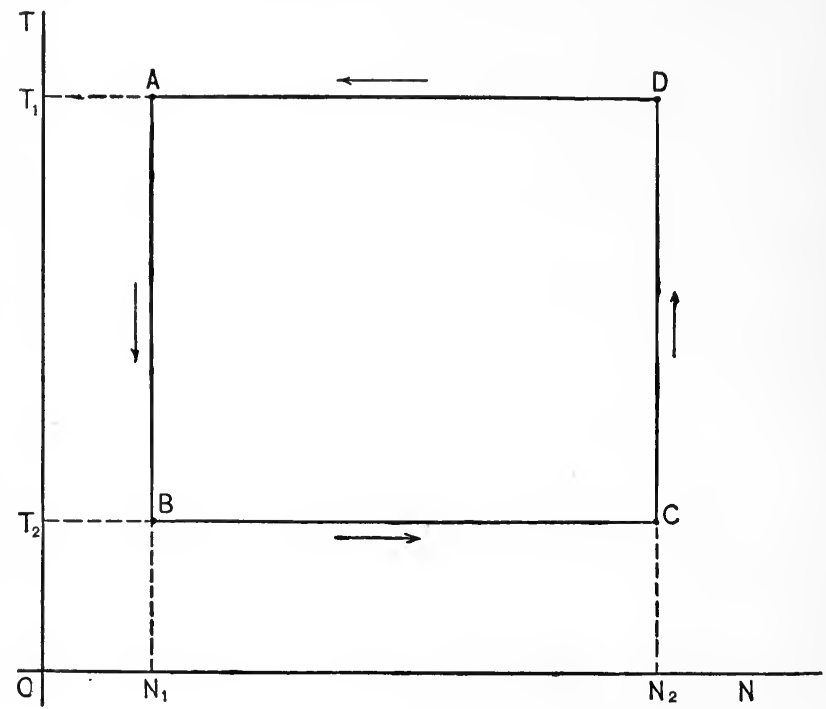

FIG. 5

that the former is now the greater, also by the area of the cycle. Plainly, there has been a net transformation of energy from what was formerly the secondary form back into the primary form.

Such a cycle is called a reversed cycle, and all of the arguments and laws which were developed for the clockwise cycle now apply as rigidly to the reversed cycle if the terms be properly interchanged to fit the case. For instance, referring to the Third Law of Thermodynamics, 
page 53 , it will be noticed that the word positive therein must now be changed to negative.

In reality, such a cycle should properly be called an "obverse" cycle; for it is merely the portrayal of the cycle of the secondary form of energy instead of the primary. For every "reversed" or counter-clockwise cycle of a secondary form of energy which takes place there must exist a corresponding "direct" or clockwise cycle on the part of a primary form to furnish it motive power. In short, -

A clockwise cycle always portrays the change taking place in a primary, a counter-clockwise in a secondary, form of energy.

This is a direct corollary of the Second Law of Energetics.

In thermodynamic work the reversed cycle is that embodied in the refrigerating or artificial-ice machine. Such a machine absorbs heat along the lower temperature-level $C B$, from the beef to be cooled or from the water to be frozen; it lifts this heat up-temperature against its will, up the adiabatic $B A$; it rejects it at the upper level $A D$ into the absorbent circulating-water; the remnant of unrejected heat at $D$ falls down-temperature to $C$. This cycle requires mechanical power to maintain it in motion. Such power is usually derived from a steamengine.

Efficiency of the Cycle. - It is to be noted, as of especial importance, that if the cycle be rectangular, as drawn in Figs. I and 2, the efficiency of conversion of energy from one form to another is given by the ratio between the temperature-range within the cycle and the temperature of the supply of heat. The energy supplied is measured by the area beneath $D A$, the curve of supply; the 
energy transformed is the area of the cycle, or $D A B C$. Therefore

$$
F=\frac{D A B C}{\text { area beneath } D A}=\frac{\text { fall in intensity utilized }}{\text { total intensity }}
$$

This gives, for weight-engines,

$$
F=\frac{H_{1}-H_{2}}{H_{1}}
$$

and for heat-engines, $\quad F=\frac{T_{1}-T_{2}}{T_{1}}$.

This equation is of fundamental importance, and permits us to deduce the broad law :-

In any rectangular cycle of energy-transformation the portion of the primary energy supplied which is converted into secondary is given by the proportion existing between the range of primary intensity during the cycle to the initial intensity of supply.

When the two forms of energy concerned in the rectangular cycle are heat and work respectively, the above principle becomes

\section{The Fourth Law of Thermodynamics}

In a heat-engine working on the rectangular cycle, the heat converted into work is to the heat supplied as the cyclical range in temperature is to the original absolute temperature of supply.

The Efficiency of the Reversed Cycle. - Approached mathematically, the quantities occurring in a reversed rectangular cycle similar to Figs. I and 2 must bear the same relation as they do therein. It is not possible, however, to deduce therefrom an expression for its efficiency in the 
usual sense, similar to equations (I3) and (14), because the energy transformed is not now a portion of the energy supplied along the lower level. It may obviously bear any imaginable relation to the latter, according to conditions of relative levels of supply and rejection. If the cycle be rectangular the heat absorbed from the body to be chilled, or $Q_{2}$, must bear the same ratio to the energy to be supplied in lifting this heat to the $T_{1}$-level, or $Q_{w}$, as $T_{2}$ bears to $T_{1}-T_{2}$. This ratio may obviously be anything whatever. ${ }^{1}$

Comparative Inefficiency of Cycles other than Rectangular. - Bringing into the argument the law proved on page $5 \mathrm{I}$, that the heat handled is measured by the area beneath the curve representing the heat-process, the Third Law will be found not to apply when the cycle is any other than rectangular. Thus, let EFGHIKLM, Fig. 6, be any rectilinear, but not rectangular, cycle. It may be considered as made up of a series of triangular departures from the circumscribing rectangle $D A B C$. In such a cycle all of the heat must be supplied along the line $E F G H$; for only during this portion of the cycle is the entropy increasing. The abstraction of heat must occur only along the line $I K L M$, for here alone is the entropy decreasing. Therefore $Q_{1}$, the heat supplied, is measured

${ }^{1}$ Herein appears the fallacy of a frequently recurring error in publications concerning artificial ice-making and refrigerating. Thus, it is often stated that, since it requires a certain number of B. t. u. to melt a certain quantity of ice into water at a given temperature, to make ice again from that same water would require, in a perfect refrigerating machine, this same number of B. t. u. But between these two quantities of heat: (I) The heat required to melt the ice, which when once in, must be lifted out again in order to freeze it; and (2) The energy required to do this lifting, there is no direct connection. If the heat had to be lifted to a high temperature, as might be the case in India, a perfect machine might require much more than this amount of heat. If it had not to be lifted far, as would be the case in Canada, a quite imperfect machine might require less energy to operate it. 
by the area $d E F G H a ; Q_{2}$, the heat abstracted, is measured by the area $a I K L M d$. Hence the efficiency,

$$
F=\frac{Q_{1}-Q_{2}}{Q_{2}}=\frac{E F G H I K L M}{d E F G H a} .
$$

Comparing the values for this equation with those for the circumscribing rectangular cycle between the same limits, $D A B C$, it is plain that $Q_{1}$ has been diminished and

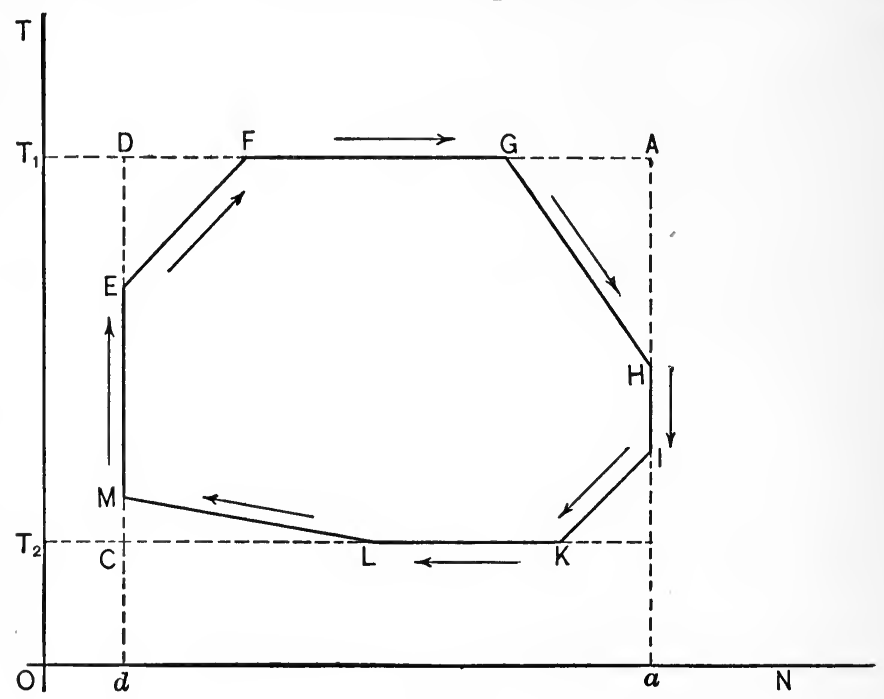

FIG. 6

$Q_{2}$ increased. The effect of either of these changes alone, or of both in combination, is to diminish the value of the fraction $\frac{Q_{1}-Q_{2}}{Q_{1}}$, which expresses the efficiency of the rectangular cycle.

It is next important to note that:-

Between any two temperature-limits the only possible irregular clockwise cycle is one lying wholly between those limits. 
Thus, in Fig. 6 it is quite possible to imagine the irregular clockwise cycle EFGHIKLM operative between the limits $T_{1}$ and $T_{2}$; for the heat can readily fall into the carrier from $D F$ as it passes along $E F$, and from $G A$ as it passes along $G H$, or out of the carrier into $B K$ as it passes along $I K$, etc. But if we attempt to imagine the cycle EFGHIKLM of Fig. 7 operative in a clockwise

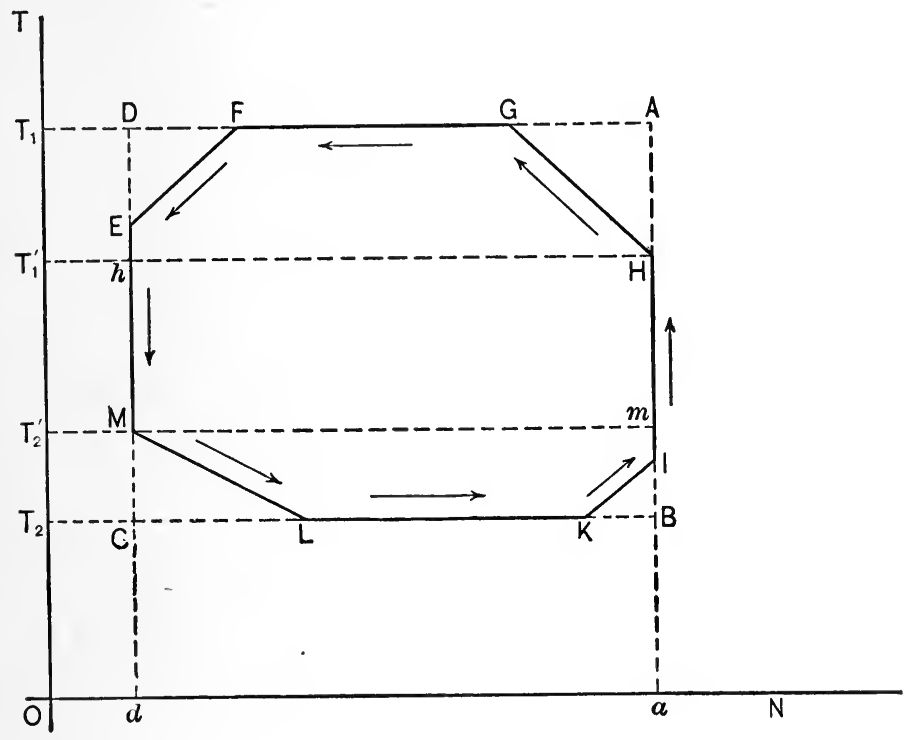

FIG. 7

direction between the limits $T_{1}^{\prime}$ and $T_{2}^{\prime}$ it becomes obvious that the thing is impossible. The carrier cannot pass along $h E F G H$ absorbing heat from $h H$; it cannot pass along $m I K L M$ rejecting heat into $m M$. The Second Law forbids.

The irregular counter-clockwise cycle MLKIHGFE of Fig. 7 is quite possible, however, between the limits $T^{\prime}{ }_{1}$ and $T_{2}^{\prime}$. The carrier, as it passes along $H G F E$, can easily 
reject heat to $H h$; as it passes along $M L K I$ it can absorb it from $M m$. Therefore,

Between any two temperature-limits the only possible counter-clockwise cycle is the rectangular one; any irregular one must vertically circumscribe that rectangle.

But in such circumscribing cycle the energy required to maintain operation is the area of the cycle. The effective result is measured by the inscribed rectangle. The efficiency of the former is plainly less than what it would have been had it been rectangular.

By assuming these triangular departures from the rectangle to become infinite in number, the proof can be extended, with the aid of the calculus, to cover a cycle of any non-rectangular form, such as $A B D C$, Fig. 4. It therefore follows, from pages 60, 61, and 62, that -

Between any two given temperature-limits the rectangular cycle is the one of maximum efficiency.

This proposition may also be proven from general considerations. If the temperature at which heat enters the engine be at any time lower than that of the heat-supply, the heat must fall into the engine unrestrictedly. If at any time the temperature at which heat leaves the engine be above that of the refrigerator, or the source of cold, heat must fall out of the engine into the refrigerator. But since the sole source of mechanical work lies in the fall of heat in temperature, it is obvious that any free or unrestricted fall of the heat down-temperature must represent a lost opportunity. The efficiency of the process must therefore be less than when no such free fall takes place. Such a lost opportunity is commonly called a "free fall" of heat.

Conditions for Efficiency. - It is said of water-wheels, and is proven by deduction from a line of argument exactly parallel to the above, and also by experiment, that the 
attainment of the maximum possible efficiency depends upon -

I. The admission of the water at the head-race level and without velocity or impact;

II. The discharge of the water at tail-race level without velocity;

III. The handling of the water between the two levels without leakage.

Similarly, it is truly said of heat-engines that the conditions of maximum possible efficiency are-

I. The admission of all of the heat isothermally at the temperature of supply;

II. The discharge of all of the heat isothermally at the temperature of the refrigerator; and

III. The vertical or adiabatic transfer of the heat (i.e. without gaining or losing any heat on the way) from one level to the other.

A heat-engine which receives heat from the source of supply after having started down the path to the lower level (as, for instance, through the reheating coils supplying live-steam heat to an intermediate receiver) is like a water-wheel with a leaky flume which lets water dribble into the buckets when they are halfway down to the tailrace ; the process is illustrated in $G H$, Fig. $6 .{ }^{1}$ The evil of beginning to drop the temperature of the heat before it has all safely arrived in the engine, as by wire-drawing, belongs to the same class.

The line $I K$ of Fig. 6 illustrates the loss due to drop-

1 It is an incidental result of the use of reheater coils that another much larger loss is modified thereby, and therefore the advisability of their use remains an open question; but it is none the less true that the fall in temperature of the live-steam heat to the temperature of the receiver must ever be an irremediable loss, which may or may not be counterbalanced by its beneficial effect upon other inevitable free-fall losses incurred at other points in the process. 
ping the heat out of the engine into the refrigerator before it has reached the lower limit by vertical, rectilinear fall, as in an engine working with incomplete expansion; it is like pouring the water out of water-wheel buckets before they have reached the level of the tail-race.

The line $L M$ shows the evil of beginning to raise the buckets before they are completely emptied; it has no familiar parallel in the steam-engine.

The line $E F$ shows how work is lost by pouring water into the buckets as they are rising; the corresponding loss in the steam-engine occurs in the heating of the feed-water in the boiler.

The rectangular cycle was first defined and its efficiency established by Carnot. It is therefore known as the Carnot Cycle. Because of its high theoretic efficiency it has been much sought after. In nearly all such search the limiting portion of the law of its efficiency, the opening words in the above statement, "Between any two temperaturelimits," has been entirely lost sight of. The fact was overlooked that higher efficiency might be attained as well or better by extending the temperature-limits than by seeking the rectangular form within certain prescribed limits. For the rectangular Carnot cycle is difficult to reproduce in practice. In fact, it has always proven impracticable of incorporation into an actual working engine. While the task might be possible of solution, yet it is certain that the expense which it would involve would always more than offset any thermodynamic gain which would ensue.

Nevertheless, the study of the Carnot cycle is of prime importance in heat-engine design; for of all of the myriad of variations which may be played by the individual designer upon the dozen known heat-engine cycles, the thermodynamic value of each can be safely and accurately 
measured only by its approach to or departure from the rectangular cycle of Carnot. And while the commercial value of a design may be quite another thing from its thermodynamic value, yet the latter must always enter into the question as a factor of prime importance whose estimation by guess is by no means easy or safe. 


\section{CHAPTER III}

\section{THE THERMAL PROPERTIES OF MATTER}

According to the molecular theory, all matter is made up of a vast number of minute particles, or molecules, each of which possesses all of the chemical characteristics of the substance in question. As to the exact size of the individual molecule, there is, of course, much difference of opinion; but in view of their infinitesimal size in proportion to any dimensions which the eye can perceive, even with the aid of the microscope, the various estimates agree more closely than might be expected. One authority offers the simile that if a pea should be magnified to the diameter of the earth each of its component molecules would have attained the size of an orange; or in other words, that the average molecule possesses a diameter of about one-billionth of an inch.

The individual molecules of a body are supposed to be separated by distances very great in comparison with their diameter, and in gaseous matter these distances of separation have been likened to those of the solar system in comparison with planets composing it. At any rate, the component particles or molecules of a body are anything else than rigid, quiescent grains; they have more or less liberty to move actively about, and they are always doing so. Balanced in equilibrium between the mutually attractive forces, called collectively cohesive, and the separative forces due to the momentum of their own motion, like the earth between its gravitation toward the sun and its 
centrifugal tendency to fly off its orbit on a tangent, they are pictured as being in constant vibration, back and forth, at all times and in all conditions of matter.

Of the form, construction, and attributes of the individual molecule little is known, even to the degree of safe conjecture. Since they must be in constant collision during their vibrations, and as we can conceive of impact only as a lack of elasticity which prevents complete restitution of mechanical energy when two bodies meet, we are forced to conceive the molecule as being absolutely and perfectly elastic; for if it were not so, some of its kinetic energy, when in collision, must be returned as heat instead of as motion. But as our only conception of heat is this same molecular motion, this is a reductio ad absurdum.

This does not necessarily imply, however, that they are simple units of matter, - homogeneous spheres, or anything of that sort. On the opposite, while knowing very little as to their absolute construction, we are forced to believe that it is very complex. One scientific authority makes use of the simile that "compared to a molecule a grand piano is simplicity itself." Other authorities conceive the molecule as being a thing as complex in structure and as divisible as an astral nebula. In short, since the entire range of astronomical science reveals no limit to the bigness of the universe, as compared with human standards, there is no basis for imagining any limit to the smallness of things. In either direction the infinite and the infinitesimal stretch into the immeasurable and the unimaginable.

Each known chemical form of matter is found in at least three distinct physical forms: solid, liquid, and gaseous.

In solids the component molecules are in some way so closely bound together, and their vibrations, though still taking place, are so narrowly and rigidly limited in relation 
to one another, that the external form of the aggregation, the body taken as a whole, is capable of resisting the action of forces of considerable magnitude which may tend to deform it.

In liquids the molecules, though still held together by a mutual attraction which limits their distance of separation (in so far as internal molecular forces are concerned), they are now quite free to assume any position relatively to one another which they choose, or to roll about one another. This rolling constitutes what we call flow.

In the gaseous form the molecules are very much more widely separated than in liquids or solids, they are more actively in vibration, and they tend to separate to the widest possible degree permitted by external resistances, such as the walls of a containing vessel.

There is a condition of gaseous matter, when it is in contact with the same matter in liquid form, which is called vaporous; and the gradation from a vapor into a gas is always more or less gradual. But this does not constitute a proper basis for calling the vapor a distinct form of matter, although its characteristics may differ quite perceptibly from those of a true gas. In fact, all of the so-called "permanent" gases are nothing more than vapors very highly superheated above their boiling points.

A gas existing under so great an attenuation that each molecule travels an appreciable distance without collision with another is said to be radiant in form.

As defined in the tabular list, thermal energy, or heat, is the energy inherent in the motion of these individual molecules, yet integrated for the entire aggregation of molecules, so that we perceive it and speak of it as being the heat of the entire body. But the motion of each particle is continually and repeatedly piling itself up as a momentary 
form of potential energy by being brought to rest against molecular resistances, instantly reconverting itself into molecular motion, as does the pendulum against gravitational forces; yet, since there is always a large majority of the molecules in motion (for the rest of each is but instantaneous), and since there is another distinct molecular energy which is purely potential, this vibration of the molecules will be called the kinetic form of thermal energy. It is the energy of periodic, if not continuous, motion, contrasted with the energy of a permanent condition of separation.

It has been demonstrated, by a line of experiment and argument which cannot be repeated here, that the phenomenon of temperature is a function of the translational velocity of this molecular vibration. Since all kinds of matter are solid in form at the lowest temperatures, become liquid when heated, and finally, when heated to a still higher temperature, become gaseous, and since the cohesion of a solid is much greater than that of a liquid, and that of a gas the least of all, it is easy to conceive of the melting of a solid as a breaking-down of the restricting cohesive forces by excessive velocity of vibration, and of the vaporization of a liquid as the same phenomenon carried to a further degree. The velocity of vibration of the molecules of the solid, as it is heated more and more nearly to its melting-point, may be imagined as putting a greater and greater strain upon the molecular, cohesive forces which bind the molecules together in a rigid connection, until finally those forces are first balanced and then overcome, and the molecules take up that freer activity which proclaims the substance a liquid. The vaporization of a liquid into a gas presents a similar series of conditions of equilibrium.

The situation is by no means so simple as has just been 
portrayed, and the student must not expect this explanation to clarify all of the puzzling problems of physics. It is perfectly certain that the thermal motion of matter must include and combine several sorts and degrees of motion, in the so-called "different degrees of freedom," involving different portions of the mass of matter present in the molecule. But for the purpose of gaining a clear general conception of how energy is absorbed by water in its variations in temperature and its transformations into ice and steam, and of how such energy may be nothing more than mass in motion, the above will be found to be a material aid.

If heat be kinetic energy of molecular translation and temperature be a function of the velocity of the same, then it is obvious that the temperature of a body cannot be increased without the addition of energy from without. This is true. A body can be warmed by rubbing it, by pounding it, by compressing it, or by simply heating it. In either case the velocity and energy of its vibrations are increased by adding more motion from witho't. Moreover, a definite increase in velocity of such vibratic $n$ ought to absorb a definite amount of energy from without; and so it does. This is so true that heat is accurately measured by means of the amount absorbed by some representative substance chosen as a standard, when raised through a definite change in temperature. The substance chosen is water to the amount of one pound avoirdupois, and the rise in temperature is defined as that from 59 to 60 degrees Fahrenheit. This amount of heat-energy is called the British thermal unit (ordinarily abbreviated as "B.t.u.") and is the basis of nearly all heat-measurements in English-speaking countries.

The Mechanical Equivalent of Heat. - As it was stated, as the primary law of thermodynamics, that there must 
always be an exact equality between the amount of heat disappearing and of work appearing, or vice versa, whenever energy changed costume from one to the other, this equality must be capable of statement in terms of our chosen units of measurements. This is also true. The numerical statement is that

\section{B.t.u. $=778$ foot-pounds.}

This ratio, of I to 778, is known as The Mechanical Equivalent of Heat, and forms the foundation-stone of modern thermodynamics. Long suspected by various physicists, it remained for the Englishman, Joule, to definitely prove the fact and determine the ratio. Joule's methods were crude as compared with those of to-day, and he obtained results which varied quite widely, as we now judge accuracy. But his results were so consistent, after endless repetition, that the equivalence was proven beyond a doubt and the law of the conservation of energy placed for the first time upon a sound foundation. The ratio $\mathrm{I}: 778$ is therefore frequently referred to as "Joule's equivalent." 1

Specific Heat and Latent Heat. - If the heat absorbed by one pound of water during a rise of one degree Fahrenheit be measured at different temperatures, it will be found to be not always quite the same. Yet it remains very close to unity, varying not one per cent between $60^{\circ} \mathrm{F}$. and the boiling-point, and by barely five per cent through the entire range of temperatures common to steam-engine work. Its value at any temperature may be found by consulting the first column of the steam-table.

If one pound of ice at a temperature of $3 \mathrm{I}^{\circ} \mathrm{F}$. be heated, with the object of reducing it to water at $33^{\circ} \mathrm{F}$., it will develop that it absorbs very much more heat than 2 B.t.u. Indeed, it will be apparent that it absorbs as much heat in

1 Grindley (Trans. Phil. Soc., Vol. 194) prefers the value 774. 
this slight change of temperature as it does in afterwards being heated up to a temperature of $175^{\circ} \mathrm{F}$., or through a rise of 144 degrees. The explanation of the apparent inconsistency lies in the fact that in the first case the water has changed its physical state from ice to water. In fact, if the ice be merely melted, from ice at a temperature of $32^{\circ}$ to water at a temperature of $32^{\circ}$, without any rise in temperature whatever, it will be found that it absorbs I 43 B.t.u.

This quantity of heat is known as the latent heat of fusion. It is apparently absorbed in a separation of the molecules against the cohesive forces which bind them together, the kinetic energy added from without being converted into the stored motion of separation. The process cannot consist of a change of velocity of molecular vibration, for there is no change in temperature involved; the energy absorbed is not revealed by the thermometer. It consists of the attainment of a mean position of greater separation of each molecule, relatively to rest, which has been attained by motion against resistance, by separation against cohesion, and is therefore purely potential in its character. It is, in fact, the potential form of thermal energy.

This sort of heat the thermometer does not perceive. Neither do our nerves. We can feel that one body is hotter or colder than another, or hotter or colder than our fingers. But if ice were not harder to the touch than water, the human perceptions would not reveal the difference between ice at $32^{\circ} \mathrm{F}$. and water at the same temperature. Yet the latter possesses 143 B.t.u. more than the former. The energy involved in a change of temperature in a substance is therefore known as its "temperature-heat" or "sensible heat" or "thermometer heat," while that involved in a change in its physical structure is known as its "latent heat" (of fusion or of vaporization as the case may be). 
The first is kinetic in character; the second is potential. The first is perceptible by our senses; the second is entirely hidden from them.

So far reference has been made only to water. But the same description applies to nearly all forms of matter, except that the quantities of heat involved are quite different. Thus, if Ioo pounds of cast-iron be put into a tub containing IOO pounds of water, the iron having a temperature of $140^{\circ} \mathrm{F}$. and the water being at $50^{\circ}$, it will be found, when the two . have come to the same temperature, that they are not at $95^{\circ}$, as might be expected, but at $60^{\circ}$. In other words, instead of the iron having fallen $45^{\circ}$ in temperature while heating the water $45^{\circ}$, as would seem natural, the iron has fallen $80^{\circ}$, while the heat which it has lost has sufficed to raise the water only $10^{\circ}$. This shows that iron absorbs or gives out, during a given change of temperature, only about one-eighth as much heat as does water.

If the experiment were repeated with other substances than iron, a different result would be obtained in each case, showing that the amount of heat absorbed per degree change in temperature is different with each substance considered. This amount of heat, measured in B.t.u., is called the specific heat of the substance. A tabular list of the specific heats of various substances used in steam-engineering will be found in the Appendix.

If the amount of heat absorbed in the melting of one pound of iron, or the vaporization of one pound of alcohol, be similarly measured, the results will be different than with the melting of one pound of ice or the vaporization of one pound of water. It is therefore said that different substances possess different latent heats of fusion or vaporization, as the case may be, as well as different specific heats. A tabular list of these heat-quantities will also be found in the Appendix. 
The Formation of Steam under Constant Pressure

Pressure and Temperature. - If a pound of water be heated in an open vessel, it is found that the temperature rises continuously, as heat is absorbed, without perceptible alteration in its physical condition, until a temperature of about $212^{\circ} \mathrm{F}$ is attained. At this point, for some invisible reason, the temperature refuses to rise further. Heat may be added indefinitely, yet the water becomes no hotter. Some result is visible, however, in the formation of vapor, and if the heating be continued long enough, it will be found that the water has quite disappeared, having been completely vaporized. Simultaneously there has occurred the absorption of a very large amount of heat.

Pressure and Temperature. - If, however, the water be placed in a closed vessel and a pressure of, say, roo pounds per square inch be applied to its surface by means of an air-pump, it will be found that when the water reaches a temperature of $2 \mathrm{I} 2^{\circ}$ it does not vaporize. Instead, the temperature will continue to rise to a point between $337^{\circ}$ and $338^{\circ}$ before vaporization sets in. There is obviously some connection between the pressure upon the surface of the water and the temperature at which it will vaporize, and this connection is found to be a very rigid and exact one: The explanation is that when the molecules attain a velocity (or, in other words, a temperature) at which they would otherwise break their molecular bonds and take on a wider range of vibration, they find themselves opposed by other resistance than merely that of molecular cohesion; they are pressed together by the external pressure upon the entire mass. They therefore continue to vibrate within their prescribed limits until they attain such a higher velocity that they are able to overcome, not only the molecular forces, but the external ones as well. 
The relation between the external pressure and the temperature at which boiling takes place is not a simple one, although it is rigid and exact. It cannot be expressed accurately by any mathematical equation. For the sake of both accuracy and convenience it is customary to refer to the columns of a steam-table for its determination. The data found in the steam-table have been derived from experiments many times repeated.

Volume and Temperature. - If account be taken of the volume of steam produced during the evaporation of the water, it will be found in each case that a definite volume has always been developed by the time that one pound of water has been just entirely evaporated. This volume is called the specific volume of saturated steam. It, too, will be found to have different values under different conditions as to pressure and temperature; but under the same conditions it is always the same. This relation between pressure and specific volume, or between temperature and specific volume, is also impossible of exact mathematical statement, though in itself rigid and exact. It is therefore customary, for the sake of accuracy as well as convenience, to refer to a steam-table for each determination.

Superheat and Saturation. - If the heating of the one pound of water in the closed vessel be continued after all of the water is evaporated, it will be found that the temperature again begins to rise; and this time it will continue to rise as long as heat be added to it. Just at the point where evaporation is complete and the final rise in temperature begins, the steam is known as dry saturated steam. At any temperature above that it is known as superheated steam. At any point between the beginning of boiling and complete saturation, when the original one pound is partly water and partly steam, the steam is known as wet saturated steam. In other words, steam in contact with watcr. is 
always saturated steam and must always have a definite temperature and a definite volume when under a definite pressure. Steam separated from the water from which it was derived may have any temperature whatever at or above the boiling-point, and any volume whatever equal to or greater than the specific volume.

If heat be added to saturated steam, it will become superheated; if heat be abstracted from it, it will condense. If pressure be released from wet steam, more steam will be formed; if the pressure upon it be increased, some will condense.

Suppose that the one pound of water and steam should originally consist of $y$ pounds of steam and of $\mathrm{I}-y$ pounds of water, and that the pressure be maintained constant; if heat be added $y$ will increase and the volume will increase in proportion, but there will be no change in temperature; if heat be abstracted, $y$ will decrease and the volume will decrease in proportion, but there will be no change in temperature. It is therefore obvious that so long as the pressure remains constant the volume can be increased only by the addition of heat, vaporizing some of the water; the volume can be decreased only by the abstraction of heat, condensing some of the steam.

If the volume be maintained constant, the pressure can be increased only by the addition of heat, which is absorbed in two ways: (I) By the rise in temperature of the entire mass; (2) By the vaporization of a portion of the water, which fills the fixed volume with steam of greater density and pressure. If the volume be maintained constant, the pressure can be decreased only by the abstraction of heat, with the consequent cooling of the entire mass and the condensation of some of the steam.

In these phenomena the student will note a distinct difference of action between these steam-and-water mix- 
tures and the permanent gases which he has probably studied in his physics.

The total amount of thermal energy which is taken up by one pound of water in changing from water at $32^{\circ} \mathrm{F}$. to dry saturated steam at any higher temperature obviously consists, according to the foregoing argument, of three portions, which are entirely distinct in their origin and characteristics, viz. :-

I. The energy absorbed in increasing the temperature of the mass, or the velocity of vibration of the molecules. It is kinetic, not potential, in its character; it is purely molecular, or internal, i.e. it is dependent upon merely the relative motion of one molecule to another, and is entirely independent of any circumstances or conditions external to the body. It is called "vibration-heat," or "temperatureheat," or "sensible heat," or "heat of the liquid." It is represented by the algebraic character $(l)$, and is presented in the steam-table in the second column.

II. The energy absorbed during vaporization in separating the molecules one from another against molecular forces. It is potential, not kinetic, in character. It is purely molecular, or internal. It is called "disgregation work," the term coming from the same Latin origin as the word congregation; the latter signifies the gathering together of a flock, the former the scattering of a flock (of molecules). It is given in the steam-table in the third column, and is represented by the letter $D$.

III. The energy absorbed in separating the molecules, one from another, against the resistance due to any external pressure which may be forcing them together. It is potential, not kinetic, in its character. It is largely external, i.e. it depends upon the amount of this external force and the distance to which it is forced back. Thus, in the ordinary vaporization of water under atmospheric pressure 
it consists of the separation of a certain volume of air from the earth against gravitation, and depends in amount upon the atmospheric pressure prevailing at the given time and place. It is called the "external work." It is represented by the letter $X$, and is presented in the steam-table in the fourth column.

Internal Energy. - Upon examination it is to be seen that the first two of these three quantities of energy possess a distinct characteristic in common, viz.: they are both "purely molecular, or internal." They are therefore lumped together into a single heat-quantity which is called the "internal energy" or the "internal work" of the body. It is given the algebraic symbol $I$.

It is obvious, from the definitions just given, that the amount of this energy is independent of external circumstances. It depends solely upon the temperature, the physical state, and the peculiarities of the body in question. Thus, with steam, if there be present a certain quantity of steam at a given temperature and pressure, the amount of its internal energy can be known without any reference to the manner or the external circumstances of the steam's production, whether under constant pressure or under any other imaginable set of conditions. The total amount of heat-energy in the steam, or the amount needed to produce it from water of any given temperature, will vary with these external circumstances ; but the amount of this heatenergy which is properly to be classed as internal energy would be the same in any case. The mathematical definition of this internal energy is given by the equation

$$
I=l+D .
$$

The Latent Heat of Vaporization. - It is further obvious that the last two of the three quantities of energy also possess a distinct characteristic in common, viz. : they are 
both potential in form. They are therefore lumped together to constitute the potential portion of the heat-energy of the body under the name "latent heat," or "latent heat of vaporization." Its mathematical definition is given by the equation

$$
L=D+X \text {, }
$$

where $L$ is the latent heat of vaporization. Its values are presented in the steam-table in the fifth column.

Total Heat. - Finally, the "total heat" of the steammass, or the quantity of heat required to produce it from water at $32^{\circ} \mathrm{F}$., is given by the equation

$$
H=l+D+X=l+L=I+X .
$$

The values of $H$ will be found in the sixth column of the steam-table.

The relation between these several heat-quantities and the characteristics which connect them may best be seen by the following tabular exhibit:-

Total Heat, or

Total Thermal Energy.

Kinetic ENERgy.

Potential Energy, or

Latent Heat of Vaporization.

(I) Heat of the liquid

$=$ temperature-heat

$=$ kinetic molecular energy.
(II) Disgregation work $=$ potential molecular energy of separation against molecular forces.
(III) External work $=$ potential energy of molecular separation against external forces.

Molecular Energy, or

INTERNAL WORK.

External WORK.

Total Thermal Energy.

Having pointed out that vaporization, or its reversed process, condensation, takes place normally under fixed temperature and pressure, it is only necessary to add that 
it need not always be present in full amount. The mathematical definitions given above apply to one pound of dry saturated steam; but of each pound of water on hand it often happens in practice that only a portion has been vaporized. The result is what is called wet saturated steam, or a steam-and-water mixture. The portion of the pound which is water, still unevaporated, is usually referred to as "percentage of moisture," and the remainder the "percentage of dryness." As the vaporization proceeds, from $0 \%$ of steam to $100 \%$ of steam, the water appears to take up the heat and become vapor, molecule by molecule; and each particle of water of equal weight, in becoming vapor, takes up an equal amount of heat and produces an equal volume of steam. It therefore follows that if the latent heat of vaporization of one pound of water be represented by $L$, and the volume produced by its complete vaporization be $V$, then, if only the fraction $y$ of the pound be already evaporated, leaving $\mathrm{I}-y$ water, the latent heat of the steam-and-water mixture must be $y L$, and its volume $y V$. This statement holds true if the original volume of the pound of water be considered zero, and it is so small in comparison with the steam-volumes formed from it that this course seldom introduces a perceptible error.

Finally, if we represent all of the heat in the steam, or, more strictly speaking, all the heat required to convert it from water of a temperature of $32^{\circ} \mathrm{F}$. into wet saturation at the given temperature or pressure, by $H^{\prime}$, and call it the "total heat" of the steam-and-water mixture, we have for wet steam the equation

$$
H^{\prime}=l+y L=l+y D+y X .
$$

The Absolute Zero. - In all of these steam-table heatquantities it must be remembered that an entirely arbitrary zero of heat and temperature has been assumed, viz.: the 
melting-point of ice. But water at $32^{\circ} \mathrm{F}$. does not contain zero heat. It contains a great deal of heat, as will quickly appear if one attempts to remove the heat in order to convert the water into ice. As noted above, I43 B.t.u. can be removed before it is frozen, and the ice is then no colder than $32^{\circ} \mathrm{F}$. Moreover, this ice still has considerable heat, for this heat can be drawn out of the ice and proves itself to be heat. This process may be continued until the ice is at the temperature of $0^{\circ} \mathrm{F}$. But even here it is obvious that more heat can still be abstracted from the ice; so that the Fahrenheit zero is plainly no real zero at all, but only a starting-point from which measurements can easily be made.

So the process of heat-abstraction may be continued, while the ice continually falls in temperature as it gives up its heat, until it has reached a temperature about $46 \mathrm{I}^{\circ}$ below the Fahrenheit zero. No one has ever cooled ice, or anything else, to this point; but it is known, by lines of physical experiment and argument which need not be produced here, that when this point were reached it would be found that the ice actually contained no more heat to be abstracted, its molecules would actually be at complete rest. This point is called the "Absolute Zero" of temperature, and the total heat of a body, in its exact sense, is always the amount of heat required to bring it from a temperature of absolute zero to its given condition. But for purposes of convenience it is much better to measure all heat-quantities as a surplus above $32^{\circ} \mathrm{F}$. So the steamtables are built on this plan. If the fact of the absolute zero be kept clearly in mind, there is no objection to be found.

The Isomorphic Curve. - The mathematical definition of entropy was given (page 39) as

$$
d N=\frac{d Q}{T} \text {. }
$$


Similarly, the mathematical definition of specific heat is

$$
S=\frac{Q}{T^{\prime}-T^{\prime \prime}} \text { or } Q=S\left(T^{\prime}-T^{\prime \prime}\right),
$$

where $S$ is the specific heat of the body in question and $Q$ the quantity of the heat involved in altering its temperature from $T^{\prime}$ to $T^{\prime \prime}$. When the alteration in temperature of the body and the heat concerned therein become less than any assignable quantity, this equation becomes

$$
d Q=S d T \text {. }
$$

Equating equations (2) and ( 16 ) gives

$$
d Q=T d N=S d T \text {, }
$$

or

$$
d N=S \frac{d T}{T} \text {. }
$$

Integrating,

$$
N=S \int \frac{d T}{T}=S \log _{e} T,
$$

if $S$ be a constant, as it is very approximately with most substances. Taking limits, this becomes

$$
N-N_{0}=S\left(\log _{e} T-\log _{e} T_{0}\right)=S \log _{e} \frac{T}{T_{0}} .
$$

This is the general fundamental equation between entropy and temperature, during changes:-

(I) When the specific heat of the body remains constant, and

(2) When the body undergoes no alteration in physical state, all of the heat being absorbed in alteration of temperature.

Should the physical state be altered by addition of heat, the process is isothermal, $T$ becomes a constant, and the integration of Equation 2 gives

$$
N=\frac{Q}{T}
$$


If Equation i9 be plotted on an entropy-temperature diagram, as in Fig. 8, taking $A$ as representing the initial condition of the heat, it must reveal a curve rising toward the right, as along $A W$; for if the body be heated without change in physical state, increase in temperature must mean addition of heat, and that again must mean increase of entropy. This curve $A W$ might then be called the "curve of heating and cooling" and is frequently referred

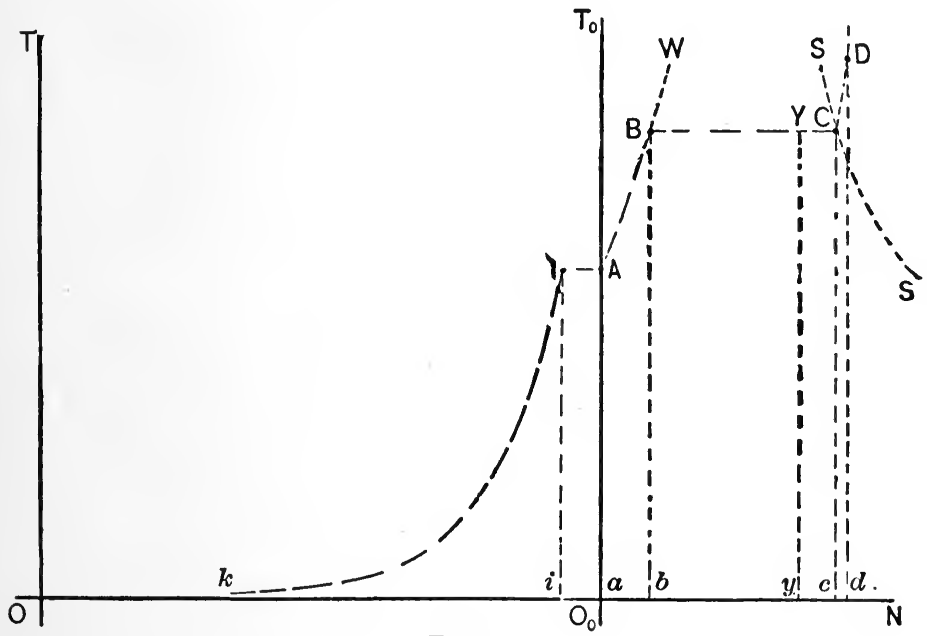

FIG. 8

to by that name; but as it is the curve on the diagram which represents heat-changes in a body only without alteration in physical state (that is, from solid to liquid, liquid to gaseous, etc.), a preferable name is the isomorphic line, by which name it will be referred to hereafter in this work. Its equation is always of the form of Equation I9:-

$$
N-N_{0}=S \log _{e} \frac{T}{T_{0}}
$$

in which the zero-subscripts refer to the original condition of the body. 
If the initial temperature of the heat-change be assumed to be zero, the value of $N$ from Equation 19 becomes infinity for any finite final temperature whatever. The conclusions forced upon us by this fact are:-

(I) That all absolute values of entropy for ordinary temperatures are very large indeed in comparison with the changes in entropy-quantity accompanying all ordinary temperature-changes; and

(2) That Equation 19, which is based upon the supposition of constant specific heat, cannot hold true for those regions of temperature adjacent to the absolute zero which have not yet been explored as to specific heats, etc.

If the point $O$, Fig. 8, represent the heat-condition of one pound of ice at the temperature of absolute zero, - that is, having no heat, no temperature, and no entropy, - and if heat be imparted to it, the process will be represented by some such line as $O k I$. The left-hand portion of the curve is indeterminate; but without question the portion near to the temperature of $32^{\circ} \mathrm{F}$. follows Equation 19, the value of $S$ being the specific heat of ice. Having attained this temperature, at the point $I$, the further addition of heat no longer increases the temperature; instead, the ice melts at constant temperature, absorbing much heat in the form of latent heat. This isothermal process is represented by the straight horizontal line $I A$.

At the point $A$, therefore, the diagram represents the thermal conditions of melted ice at $32^{\circ} \mathrm{F}$. This, for considerations purely of convenience, has been chosen as the arbitrary zero of heat for the construction of the steamtables. Water at this temperature, or $492.8^{\circ} \mathrm{F}$. absolute, is arbitrarily assumed to have no heat. In reality it has much.

Equations 17 to 20 everywhere make use of the temperature-factor as an absolute quantity and never as a relative one. The entropy-factor, on the contrary, always 
appears as a relative quantity, its absolute value being indeterminate. It is therefore quite allowable to measure all values of $N$ from a purely arbitrary zero, such as that show by the axis $O_{0} T_{0}$ through the point $A$ representing melted ice. The zero-axis of temperature, however, must always appear in its true, absolute position, as at $O_{0} N$.

If further addition of heat to the melted ice be made, the process must again follow the isomorphic $A W$, and will continue to do so until the temperature is able to overcome the resisting pressure and cause evaporation. Should this point be reached at $B$, the further addition of heat will result in the isothermal evaporation of the water, under constant pressure and temperature, along the line $B C$ to $C$, at which point it has all been evaporated into dry saturated steam. Further addition of heat again causes rise in temperature along the isomorph $C D$ of superheated steam. This curve will continue unmodified until the temperature of dissociation is reached, - a point outside the province of the present discussion.

Each of the curves $K I, A B$, and $C D$ has the same equation, viz.: Equation 19; but in each the value of $S$ is different, being in the several cases the specific heats of ice, water, and steam respectively.

The temperature-level at which the evaporation $B C$ occurs depends upon the pressure upon the surface of the water. As there may be an infinite number of such pressures, differentiated by infinitesimal steps, so there may be an infinite number of horizontal isotherms such as $B C$, separated vertically by infinitesimal temperaturesteps. The infinite number of $C$-points found in such a series would all lie in a locus such as $S S$. Each point in this locus represents the thermal condition of one pound of saturated steam of the given temperature. It is therefore known as the Line of Saturation. 
The adiabatics, as $B b, C c$, the isotherms, as $I A, B C$, and the isomorphs, as $A B, C D$, all represent natural processes frequently met with in practice. The saturation-Curve DOES NOT REPRESENT A NATURAL PROCESS. The alteration of steam up-and-down temperature while remaining in a condition of dry saturation does not occur in nature, and could be artificially enforced only by the most delicate and unstable adjustment of heat-supply or heat-abstraction during the process. The locus of saturation-points is, however, a great convenience in geometric calculations.

It is obvious that any point to the right of $S S$ must represent steam having both more entropy and more temperature than saturated steam of the same pressure; it therefore must represent superheated steam. Any point between $S S$ and $A E$ must represent a steam-and-water mixture; for it has more entropy than water and less than dry steam. Any point to the left of $k I A E$ must be meaningless (except in the strictly mathematical sense prescribed for all negative quantities); for it has less entropy than water or ice of the same temperature, which is impossible.

To this statement must be noted the unimportant exception that the exact temperature-level of the isotherm $I A$ is somewhat variable, according to the pressure under which the melting or freezing takes place. But as a wide variation in pressure produces only a slight variation in temperature, only a very slight area above $I A$ and to the right of $k I$ produced suffices to cover all of the territory where a geometrical point might represent a physical actuality.

In the passage of the pound of steam-and-water mixture across the isothermal path $B C$, from all water at $B$ to all dry steam at $C$, the vaporization takes place particle by particle, each molecule of water absorbing an equal amount of heat, an equal amount of entropy, and producing an equal 
increment of volume. This fact, stated mathematically, gives the equation

$$
y=\frac{y L}{L}=\frac{n}{N}=\frac{v}{V}
$$

where $y$ is the fractional weight of steam vaporized, $y L$ the latent heat absorbed therein, $n$ the increase in entropy due to the vaporization of $w$, and $v$ the volume of steam produced. The capital letters represent the specific quantities of the same things applying to the unit of weight. Thus, if the point $Y$, Fig. 8 , represent the heat-condition of a pound of steam-and-water mixture, then the weight of steam present, the heat and the entropy absorbed in its vaporization, and the volume produced can all be known by equating the proportion $\frac{B Y}{B C}$ to Equation $2 \mathrm{I}$; for in the latter all of the denominators can be found from the steamtables.

If attention be returned to the tabulations of energyquantities given on pages 77-79, it will be plain that many of them must be visible upon Fig. 8 in the graphical form of areas. Since the heat handled in any process is measurable by the area beneath the curve depicting the process and between ordinates (see page 50), the heat absorbed in raising one pound of ice from absolute zero to the melting-point must be measured by the area beneath the curve OkI. That absorbed in the isothermal melting of the ice must be measured by the area of the rectangle iIA $a$ beneath $I A$.

This brings consideration to the zero of the steam-tables, at $A$; for all ice-phenomena lie below its lower limit. Starting from melted ice, the heat absorbed in the raising of the one pound of water from $32^{\circ}$ to any known temperature, as at $B$, which is the heat of the liquid at that point, or the heat-quantity $l$, must be measured by the area $a A B b$. 
The latent heat absorbed in the isothermal vaporization of the one pound of water along $B C$ must be measured by the area of the rectangle $b B C c$. There are, of course, an unlimited number of such rectangles between $A W$ and $S S$, according to the temperature-level of $B C$. It is further obvious that Equation 2I, applying to any given steam-andwater mixture, such as that at $Y$, shows that the amount of latent heat present in the mixture is measured by the area $b B Y y$. This also follows from Equation 3 and from the argument on pages 50-5 $\mathrm{I}$.

The heat absorbed in superheating the steam above the temperature of saturation, as to $D$, must be measured by the area $c C D d$.

The total heat, as given by the steam-tables, from water at the temperature of melting ice to either hot water, as at $B$, to partial evaporation, as at $Y$, to dry saturation, as at $C$, or to superheat, as at $D$, must be measured by the area between $a$ and the point in question and beneath the curve $A B C D$.

In all of the foregoing illustrations and arguments it has been assumed that the substance undergoing thermal alteration was what is known chemically as $\mathrm{H}_{2} \mathrm{O}$. It is obvious that the diagram of Fig. 8 and the equations would apply equally correctly to any other substance which is known to pass successively through the stages of solid, liquid, and vaporous in a manner similar to water, provided the proper specific heats, and other constants be substituted. Such diagrams can be applied usefully in the study of the action of ammonia, carbonic acid, ether, etc., in the refrigeratingmachines, and of liquefied air, oxygen, nitrogen, hydrogen, etc., in the investigation of low temperatures. For the numerical values of the former series reference may be had to Peabody's "Steam Tables." Of the latter series very little is known empirically; nevertheless, the diagram 
is exceedingly valuable in aiding the comprehension of the rather obscure phenomena occurring in the liquefaction of the so-called permanent gases.

\section{PROBLEMS IN THE USE OF THE STEAM-TABLES}

1. If a correct steam-gage reveal a pressure of $93.7 \mathrm{lbs}$. per square inch and the barometer stand at $29.37^{\prime \prime}$, what is the absolute pressure within the gage? (One cubic inch of mercury weighs 0.49I lb.) Ans. 108. 2 lbs.

2. What is the temperature of saturated steam of an absolute pressure of $172.3 \mathrm{lbs}$. per square inch? Ans. $369.15^{\circ}$.

3. If the temperature of a body of saturated steam be $148^{\circ} \mathrm{F}$. and the barometer stand as in Problem I, what would be a correct reading for a vacuum-gage connected with the same? Ans. 22.19".

4. What is the "total heat" of the steam of Problem 2? Ans. I I 93.93 B.t.u.

5. What would be the total heat per pound of this steam were it known to be $27 \%$ wet? Ans. 963.67 B.t.u.

6. How much heat does it require to alter one pound of water from a temperature of $62^{\circ} \mathrm{F}$. to $187^{\circ} \mathrm{F}$.? Ans. I 25.5 B.t.u.

7. How much more heat is required to convert it into steam of a pressure of $84 \mathrm{lbs}$. absolute but $5 \%$ wet? Ans. 977.5 B.t.u.

8. How much more heat (after the process of Problem 7 is finished) is required to convert it into steam of the same pressure but having a temperature of $468^{\circ}$ F.? Ans. I 28.74 B.t.u.

9. How much external work is done by the heat added in Problem 6? Ans. Practically zero. It is so small a quantity that it may always be neglected in ordinary engineering calculations. If the water were heated under atmospheric pressure, it would in this case amount to about r.07 foot-pounds; under other pressures it would be proportional thereto.

10. How much external work is done by the heat added in Problem 7? Ans. 59,320 foot-pounds.

11. What would be the final volume in the case of Problem 7 ? Ans. $4.927 \mathrm{cu}$. ft. 


\section{CHAPTER IV}

\section{THE STEAM-ENGINE CYCLE}

LET Figs. 9 and Io represent rectangular coördinate fields measuring temperature and entropy in the first case, and pressure and volume in the second case respectively. Let us trace on these fields the action occurring in an ideally perfect steam-engine.

It is first to be noted that consideration of mere locality of occurrence has nothing to do with thermodynamic phenomena. Thus, in the actual steam-boiler and engine the pressure is first supplied by a feed-pump, then the heat is added in the boiler, and finally the steam is removed to the engine-cylinder for further thermal action. In following the thermodynamics of these processes, however, it is much easier if they all be imagined as occurring within the engine-cylinder; that is, that there is first placed therein a pound of cold water to which heat is applied, first warming it and developing pressure, and finally engendering steam and volume. Then let it be imagined that the source of heat be removed while the piston takes its stroke; after which condensation is carried on by the application of cold within the cylinder instead of within a separate condenser.

In order that our diagrams may agree with the steamtables, it is necessary to confine the attention to the heatphenomena occurring in a single pound of water, and to assume that the initial condition of this water coincides with the arbitrary zero of the steam-tables, the temperature 
of melting ice, or $32^{\circ} \mathrm{F}$. At this temperature, or $492.8^{\circ}$ absolute, the water is supposed to have zero heat, and therefore zero entropy. Its volume is $0.017 \mathrm{cu}$. $\mathrm{ft}$; ; but this is so small in comparison with the other volumes handled that it will not appear upon the diagram, and may be omitted from all calculations without appreciable error in engineering work. At this temperature, too, its pressure is too small for exhibition upon the diagram. Its condition is therefore properly represented by the point $A$ in Fig. 9, and very closely by the point $O$ in Fig. Io.

As heat is added, both temperature and entropy must increase along the isomorphic curve $A W$. The pressure will also increase markedly. The volume will increase slightly, but only by a very small percentage of the original volume, which is itself too small for visibility on the pressure-volume diagram. The process is therefore properly represented by the line $O C$ of Fig. IO, or, with the greatest accuracy, by a line parallel and very close to it.

The point $C$ represents, then, the thermodynamic condition of one pound of heated water under a retaining pressure which is equal to its own vapor-tension; this pressure is therefore sufficient to restrain the water from vaporization. In order to study the different processes which may develop from this point the following series of problems is presented.

The Cycle of the Boiler-explosion. - Let the point $C$ represent the condition of any single pound in a ton of hot water contained in a steam-boiler, or in any other similar vessel. For the time it is permissible to neglect the presence of any steam above the surface of the water, if there be any. Let it be supposed that a rupture of the boilerwalls suddenly releases from the water the pressure which has been upon it. The decreased pressure permits the kinetic energy of the vibrating molecules of water to 
expend their temperature in mutual separation. Vaporization and the periormance of external work ensue.

Because of the instantaneous character of the process there is no time for heat-interchange with surrounding bodies. The process therefore takes place naturally and actually as the pure adiabatic which it tends to be. Such

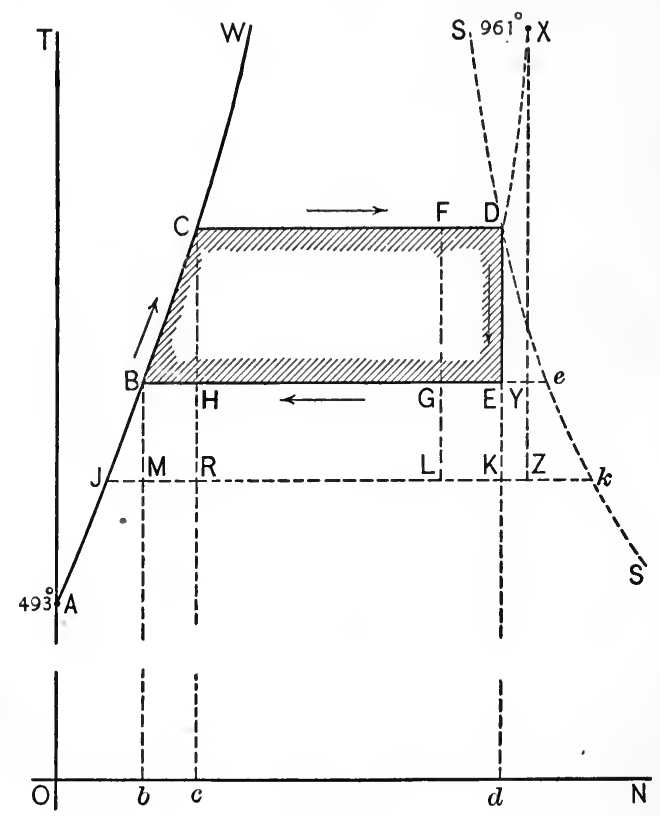

FIG. 9

an explosion is, in fact, one of the few actual instances of a purely adiabatic process known in practice.

Adiabatic fall from $C$, Fig. 9, develops the plumb-line $\mathrm{CH}$. When $\mathrm{H}$ is reached, the kinetic energy of the molecules is in equilibrium with the restraining pressure, which is now the atmospheric pressure, and further fall becomes impossible.

The fact that $H$ lies between the water-curve $B C$ and 
the saturation-curve $S S$ shows that the one pound of $\mathrm{H}_{2} \mathrm{O}$ is now a mixture of water and steam. The proportion of steam (by weight) present is given by the ratio of $B H \div B e$. The heat still present in the mixture must be that beneath the curve $A B H$. In other words, the heat which must be rejected if the water is to be returned to its original condition at $A$ must be the area beneath the curve $H B A$. The heat which has been converted into

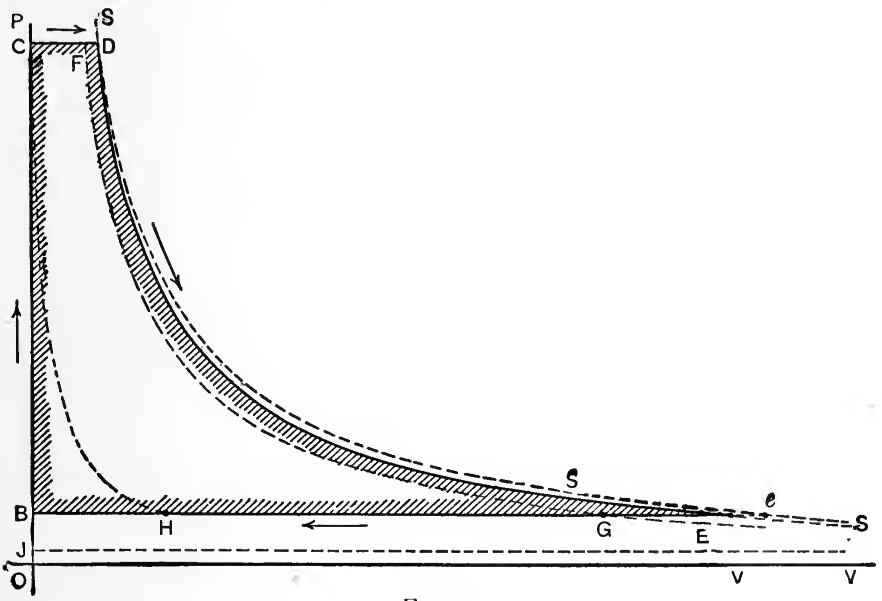

FIG. IO

work must be measured by the area between $A B C$ and $H B A$. This area is the triangle $B C H$.

Problem I2. - If a boiler containing one ton of hot water under a gage-pressure of Ioo pounds per square inch should explode, how much energy (in foot-pounds) would be released, and what volume of steam would be formed?

Let it be assumed that the barometric pressure is 29.50 inches of mercury. (In actual steam-work the barometerreading must always be known.) This locates $B$ at the level of $2 \mathrm{II} .3^{\circ} \mathrm{F}$, or $672 . \mathrm{I}^{\circ}$ absolute. The pressure at $C$, being IOO pounds per square inch higher than this, is located 
by the aid of the steam-table at the absolute temperature of $798.24^{\circ}$. The entropy of $B$ is 0.3116. That of $C$ is 0.4860. Therefore the entropy $B H$ is 0.1744.

The heat beneath the isomorphic $B C$ must be the heat of the liquid at $C$ minus that at $B$, or $308.0-\mathrm{I} 80 . \mathrm{I}=$ I 27.9 B.t.u. The heat beneath the isothermal $H B$ must be the width of this rectangle $b B H c$ times its height, or 0.1744 times 672.I, which is II7.2 B.t.u. The area of the triangle is therefore the difference between these two heats, or $127.9-$ I 17.2 , or 10.7 B.t.u.

This is the energy released per pound expressed in heatunits. The total energy released, expressed in foot-pounds, must be -

$10.7 \times 2000 \times 778=16,649,200$ foot-pounds.

This is about equal to that developed by the firing of a modern 6-inch rifle.

The question as to the volume of steam developed calls for reference to Equation 21, page 87. In applying this equation the entropy of vaporization, $B e$, at the $B H$-level, appears from the steam-table to be 1.4372 . The entropy $B H$ is 0.I744. Therefore the proportion of steam present is $\frac{0.1744}{1.4372}=0.1213$ of a pound per pound. The specific yol-

ume of vaporization at this same level is 26.98. Therefore the total volume of steam formed is -

\section{O. $1213 \times 26.98 \times 2000=6547$ cubic feet.}

This explains why boiler-explosions are so completely destructive to the buildings enclosing them. The building explodes, as well as the boiler, from the immense volume of steam formed. The action is so instantaneous that no weakest point has time to relieve the rest of the structure by giving way first. Each brick is torn from its neighbor, and the building collapses into a heap of rubbish. 
The Cycle of the Perfect Steam-engine. - Returning to the store of heated water with which the boiler-explosion began, at the point $C$, let it now be supposed that the boilerwalls did not give way, but that instead heat continued to be absorbed by the water. The temperature of the water being already in equilibrium with the pressure upon it, further advent of heat must develop isothermal vaporization. The entropy will increase along the isothermal $C D$ (Fig. 9) and the volume will increase along the constantpressure line $C D$ (Fig. Io). At $D$ vaporization is complete, and the steam is dry and saturated.

In the actual engine, at this stage of the process, the steam leaves the boiler and passes to the engine-cylinder, where $D$ represents in the actual engine the point of cutoff. Here the steam finds itself isolated from all heat-interchange with the rest of the universe but free to impart its molecular energy to the piston in the shape of mechanical work, because the intensity of its kinetic energy exceeds the resisting pressure of the piston.

Finding therein an opportunity to drop in temperature, it promptly does so, falling down the adiabatic $D E$ (Fig. 9) in the process. At $E$ further fall becomes impossible. In the case of the non-condensing engine, the limiting factor is atmospheric pressure; in the case of the condensing engine it is sometimes the pressure and sometimes the temperature in the condenser; that is, either a poor air-pump or a warm condenser will limit the vacuum.

In order to leave the cylinder ready for a repetition of the process the steam in it must be condensed at this bottom temperature-level. The condensation therefore takes place under constant pressure, along the isothermals $E B$ (Figs. 9 and IO), until it is complete at $B$. The pound of water is then ready for a repetition of the cycle $B C D E$. 
By the application of Equation $2 \mathrm{I}$ to any point of $D E$ and $B H$ in the same way as was done to the point $H$ of the boiler-explosion the curves $D E$ and $B H$ of Fig. Io can be developed. The cycle is then represented in the pressurevolume diagram by the same circuit $B C D E B$, in which $C D$ and $E B$ are isothermals and $D E$ is an adiabatic.

This cycle of processes is called the Cycle of the Perfect Steam-engine, or the Rankine Cycle.

It will be noticed, first, that the adiabatic process $D E$, when represented in the pressure-volume diagram, results in a hyperbolic curve instead of a perpendicular drop. This shows that the adiabatic process is a heat-process, not a steam-process or a volume-process ; for the straight line of fall is the natural one. Indeed, the pressure-volume effects of adiabatic drop upon the various vapors and gases are the most diverse; whereas when viewed simply as heatprocesses these diverse adiabatics always appear as vertical plumbline drops.

In the second place, it should be noted that its effect upon the volume and pressure of steam-vapor is so complex as to defy exact mathematical expression. The curve $D E$, Fig. Io, is hyperbolic in character, as are all those near it, and asymptotic to the two axes; but there is no known exact equation for it. The only exact, and the most convenient, method of deriving it is the graphical one through the medium of the corresponding curve in the entropy-temperature diagram described above.

Problem 13. What are conditions of operation and the efficiency of a perfect steam-engine operating between a boiler-pressure of Ioo pounds per square inch by gage and exhausting into the atmosphere? Represent its operation by means of entropy-temperature and pressure-volume diagrams.

The points $B$ and $C$ have already been located in con- 
nection with Problem I2. The entropy of $C$ appears from the tables to be 0.4860 and that of $D$, to be 1.5837. The entropy of $E$ being the same as that of $D$ and that of $B$ being 0.3116 , the entropy $B E$ appears to be

$$
\mathrm{I} .5837-0.3 \mathrm{II} 6=\mathrm{I} .272 \mathrm{I} \text {. }
$$

The specific entropy $B e$ at this level being I.4372, the ratio of the two entropies gives the steam-weight present as $0.885 \mathrm{I}$ pound. The specific volume of steam at this pressure being 26.98 cubic feet, the volume of the wet steam after expansion to $D$ must be

$$
0.885 \mathrm{I} \times 26.98=23.88 \text { cubic feet. }
$$

If the cycles of Problems I 2 and 13 as portrayed in Fig. 9 be compared, it appears that the latter is made up of the triangle of the former, $B C H$, and the rectangle $H C D E$. The heat supplied in Problem I 3 is greater than that of Problem 12 by the rectangle beneath $C D$; the heat rejected is greater by the rectangle beneath $H E$; the heat converted into work is greater by the rectangle $H C D E$. The heat supplied is

$$
Q_{1}=l_{1}-l_{2}+L_{1}=\text { I004.4 B.t.u. }
$$

The heat rejected is the entropy $B E(=B H+C D)$ times the absolute temperature at $E$, or

Therefore

$$
Q_{2}=\left(N_{D}-N_{B}\right) T_{2}=855.0 \text { B.t.u. }
$$

$$
Q_{w}=Q_{1}-Q_{2}=\mathrm{I004.4}-855.0=\mathrm{I} 49.4 \text { B.t.u. }
$$

The efficiency of conversion of heat into work, or the thermodynamic efficiency, is

$$
F=\frac{Q_{1}-Q_{2}}{Q_{1}}=\frac{149.4}{1004.4}=14.87 \% .
$$


If the area of the triangle which measures the heat converted into work in the boiler-explosion cycle be expressed analytically, there results

$$
l_{1}-l_{2}-T_{2}\left(N_{C}-N_{B}\right)=l_{1}-l_{2}-T_{2} S \log ^{e} \frac{T_{1}}{T_{2}}
$$

If the area of the rectangle $H C D E$ which is added to this in making up the Rankine cycle be expressed analytically, there results

$$
\left(T_{1}-T_{2}\right)\left(N_{C}-N_{B}\right)=\left(T_{1}-T_{2}\right) \frac{L_{1}}{T_{1}}
$$

If these be added, there results

$$
Q_{w}=l_{1}-l_{2}-T_{2} S \log ^{e} \frac{T_{1}}{T_{2}}+L_{1}\left(\frac{T_{1}-T_{2}}{T_{1}}\right) .
$$

This is the algebraic expression for the utmost work attainable from dry steam between the limits $T_{1}$ and $T_{2}$. It is inexact in that $S$ is not a constant over any wide range of temperature. The calculation of the graphical areas by means of the steam-tables is therefore to be preferred, for accuracy as well as for convenience. There may arise occasions, however, when the above expression is useful. With a proper choice of value for $S$ its inaccuracy is not great.

The rèctangle $H C D E$ must represent a Carnot cycle, of the maximum efficiency, given by the expression $\frac{T_{1}-T_{2}}{T_{1}}$. The last term of Equation 22 is easily recognizable as arising from such a cycle, $L_{1}$ being the heat supplied along the isothermal $C D$ and the parenthesis being the efficiencycoefficient by which it is reduced to work done. It will also be noted that the general contour and the efficiency of the Rankine cycle $B C D E B$ closely approach those of the Carnot cycle $H C D E H$. In Problem I $3,14.88 \%$ of the heat was converted into work. Had the Carnot cycle 
been followed between the same temperature-limits, the portion of heat converted into work would have been

$$
(798.3-672.1) \div 798.3=15.81 \% \text {. }
$$

The difference is not worth striving for. Here can be seen for the first time what will be frequently referred to again: That the inefficiency of the steam-engine is due neither to a poor cycle nor to an improper working-substance, but to narrow temperature-limits. For its cycle is a close approach to the ideally perfect rectangle.

Problem I4. If the engine of Problem I 2 were fed with steam $20 \%$ wet, what would be the efficiency, etc.?

In this case the boiler-steam, instead of being completely evaporated to dryness at $D$, leaves the boiler with one-fifth of its weight still unevaporated water. Its condition is shown at the point $F$. The expansion now follows the adiabatic $F G$, and the complete cycle is $B C F G B$. At $F$ its entropy is $0.4860+(0.8 \times 1.0977)=\mathrm{I} .3642$; its volume is $0.8 \times 3.88 \mathrm{I}=3.105$; its total heat is

In this case

$$
308.05+(0.8 \times 876.35)=\text { 1009. } 1 \text {. }
$$

$$
Q_{1}=\text { I009. I }-l_{2}=\text { I009. I }- \text { I } 80.1=829.0 \text { B.t.u. }
$$

The entropy of vaporization at $G$ is

$$
\text { I. } 3642-0.3 \text { I I } 6=\mathrm{I} .0526 \text {; }
$$

the specific entropy of vaporization at this level being I.4372, the steam-weight present at $G$ is given by the ratio between these two, or

$$
\frac{\mathrm{r} .0526}{\mathrm{r} .4372}=0.7324 . \quad 100-73.24=26.76 .
$$

The initial $20 \%$ of water has become $26.76 \%$ during expansion.

In this problem $Q_{2}$ is the area of the rectangle under 
$B G$, or $965.9 \times 0.7324=707.42$. Hence the work done is $829.0-707.42=\mathrm{I} 2 \mathrm{I} .58$ B.t.u., and the efficiency is $14.66 \%$.

In actual practice the deleterious effect of wet steam is much greater than that shown by a comparison of these two problems. The trouble is not that the water diminishes the theoretic possibilities for the development of work, but that its presence interferes with the realization of those possibilities, in a manner to be described in Part II.

Problcm I 5. If the engine of Problem 13 were to exhaust into a condenser maintaining a vacuum of $25^{\prime \prime}$ of mercury instead of into the atmosphere, what would be the efficiency, etc. ?

Here the lower temperature-limit to which adiabatic drop may take place has been lowered by the decreased backpressure to $130^{\circ} \mathrm{F}$., or $590.8^{\circ}$ absolute. This temperature-level is represented by the line $J K$ (Fig. 9). All of the quantities can be calculated as before.

Problem I6. Find the results of exhausting the engine of Problem I4 into a vacuum of $25^{\prime \prime}$.

Problem I 7. If the engine of Problem 13 were to be fed with steam superheated to a temperature of $500^{\circ} \mathrm{F}$., the pressures remaining the same, what would be its efficiency, etc.?

In Figs. 9 and Io the point $D$ represents steam just at the point of saturation, to be superheated only by additional heat. The addition of this heat will increase both temperature and entropy according to the equation

$$
N-N_{D}=S \log _{e} \frac{T}{T_{D}}
$$

where the subscripts indicate the point $D$ and the value of $S$ is the specific heat of steam, or about 0.55 . This process is shown by the curve $D X, X$ being the condition of the steam in the engine just before expansion begins. The 
line $D X$ in the $P V$-diagram would be a straight horizontal extension of $C D$, for the superheat causes the volume to increase under constant pressure. Both $D X$ and the expansion-curve $X Y Z$ are omitted from the $P V$-diagram, to avoid confusion, for the latter would be a hyperbolic curve lying close to and crossing $S S$ at a very sharp angle. The efficiency and any volumes desired can be investigated and calculated with the help of the entropy-temperature diagram alone. ${ }^{1}$

When cut off from the boiler the steam-heat at $X$ promptly drops in temperature down the adiabatic $X Y$ to $Y$, at which point no further drop is possible. The rest of the heat must be abstracted along $Y B$. The increase in entropy due to $D X$ is found from the above equation to be $E Y=$ 0.1020. Taking the value of 1.272I for $B E$ from Problem I 3, $B Y=B E+E Y=1.272 \mathrm{I}+0.1020=1.374 \mathrm{I}$. Since $B e=\mathrm{I} .4372$, the steam-weight present at $Y$ must be $0.949 \mathrm{I}$ pound. The $Q_{1}$ of this problem must be greater than that of Problem $\mathrm{I} 3$ by the heat added along $D X=0.55(500-337.4)$ $=89.4$ B.t.u. $Q_{1}=$ ro93.8. The $Q_{2}$ must now be greater than that of Problem I 3 by the rectangle under $E Y$, or it may be determined directly as the area beneath $B Y$; whence $Q_{2}=923.5$. Therefore $Q_{w}$, the work done, is $Q_{1}-Q_{2}=1093.8-923.5=170.3$ B.t.u., and the efficiency of the cycle is $\frac{\mathrm{I} 70.3}{\mathrm{I093.8}}=\mathrm{I} 5.6 \%$.

The beneficial effect of superheat upon the efficiency in the actual engine is much more than what is shown by comparison of this result with Problem I3. The reasons for it are the exact reverse of those processes which make

1 The close confusion of these several diverse curves in the $P V$-diagram, although they are entirely distinct in the $N T$-diagram, stands witness to the obscurity of the evidence as to what is thermally transpiring in the cylinder which is offered by the indicator-card. 
wet steam so deleterious, and will be explained in the Second Part of this work.

Problem 18 . What would be the efficiency and conditions of operation of a direct-acting steam-pump working non-expansively between a boiler-pressure of 100 pounds by gage and the atmospheric pressure?

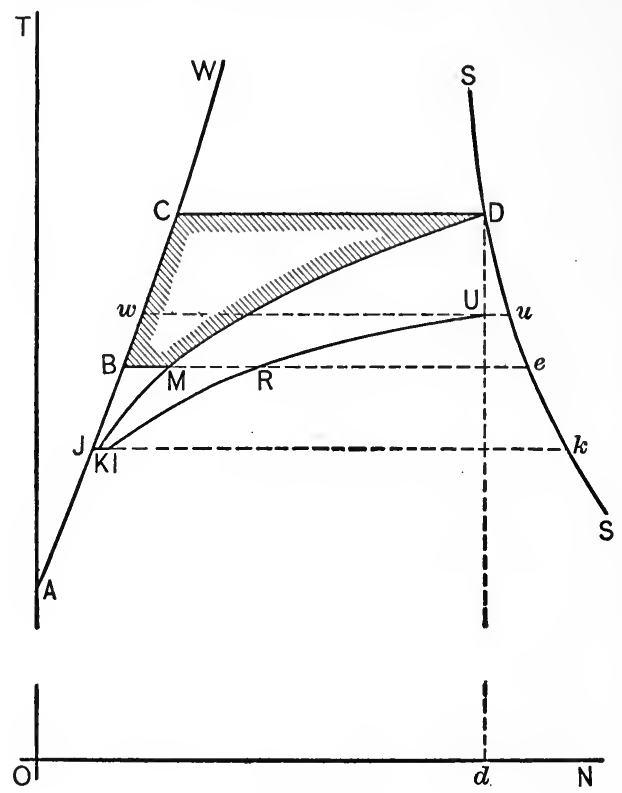

FIG. II

Again, the point $D$ (Figs. I I and I2) represents the condition of the steam-heat as the supply from the boiler is cut off in the pump-cylinder. But now, instead of being allowed to expand adiabatically, the heat is abstracted at constant volume while the piston stands still at the end of the stroke. In the actual pump the exhaust-valve is opened at the end of the stroke to a separate condenser, but thermodynamically the abstraction of heat by the condenser 
may better be imagined as taking place entirely within the cylinder.

The heat is now not free to fall naturally down the adiabatic $D U$, but is diverted artificially by the limitations of the case into following the path $D M$, called the constantvolume curve, the heat meanwhile dripping from the various points on this curve down to the tail-race level $B e$ by means of "free fall." The curve $D M$ has no known exact

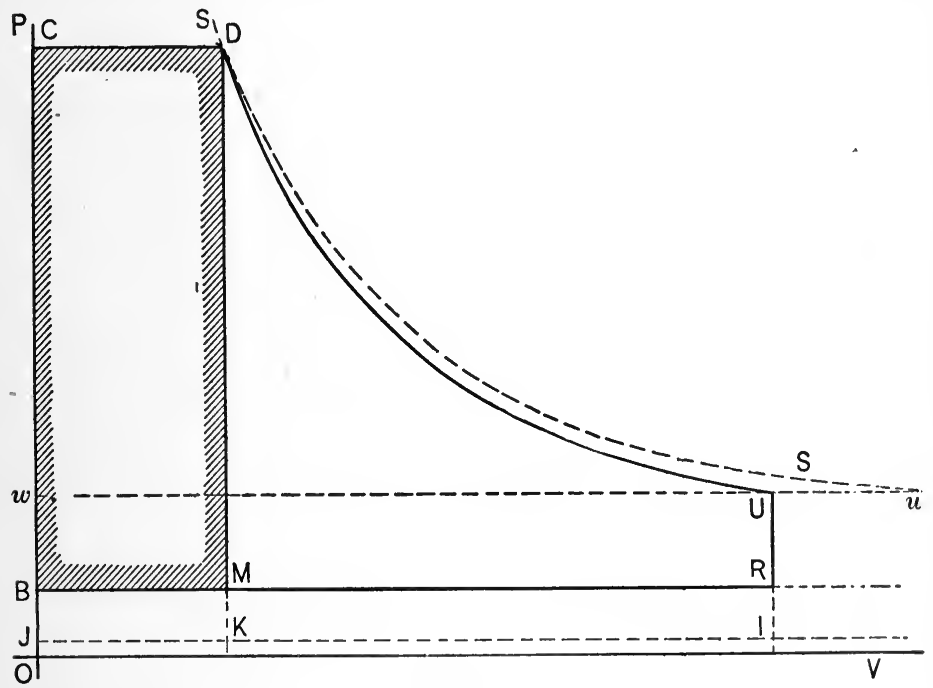

FIG. I2

equation in the $N T$-diagram ; in the $P V$-diagram its equation is $V=$ a constant. But the $N T$-curve may be found by determining individual points by the help of Equation $2 \mathrm{I}$. In this case it is the volumes which are known and the other variables which are to be found. Thus, at $M$ the volume is the same as at $D$, or $3.88 \mathrm{I}$ cubic feet. At this level the specific volume is 26.98 cubic feet. Therefore there must be present a steam-weight of $\frac{3.88 \mathrm{I}}{26.98}=0.1438$ pound. Its 
entropy of vaporization is $0.1438 \times \mathrm{I} .4372=0.2067$. Its total entropy is $0.5 \mathrm{I} 82$, which locates the point $M$ in the $N T$ diagram. Intermediate points on the curve $D M$, Fig. I I, may be found similarly.

In this problem the $Q_{1}$ is the same as in Problem I3, or I004.4 B.t.u. $Q_{2}$ cannot be found directly from the NT. diagram, but can be deduced from the value of $Q_{w}$ given by the $P V$-diagram. The area of the rectangle $B C D M$, Fig. I 2 , is $100 \times 144 \times 3.88 \mathrm{I}=55886$ foot-pounds. ${ }^{1}$ Therefore the area of $B C D M$, Fig. II, is $\frac{55886}{778}=7 \mathrm{I} .83$ B.t.u. This shows an efficiency of $\frac{71.83}{1004.4}=7.1 \%$.

Problem 19. What would be the efficiency, etc., were the pump of Problem 18 to exhaust into a vacuum of $25^{\prime \prime}$ ?

Here the cycle describes the path $J C D K J$.

Problem 20. What would be the efficiency, etc., of the engine of Problem I 3 if, instead of carrying its expansion completely to atmospheric pressure, it should have reached the end of its stroke by the time the steam had expanded to a gage-pressure of 20 pounds and the exhaust-valve had then been opened?

The cycle now describes the path $B C D U R B$ (Figs. I I and I2), the wu-level being at the temperature corresponding to saturation at 20 pounds by gage, or $258.2^{\circ} \mathrm{F}$., or $719^{\circ}$ absolute. The cycle may be divided into two distinct portions: (I) $w C D U$ and (2) BwUR. The first portion can

1 In calculating quantities of work from volumes and pressures of fluids, whether the latter be steam, water, air, or other gases or liquids, it is engineering custom for volumes to be always measured in cubic feet and pressures in pounds per square inch. At the same time the resultant work is customarily desired stated in foot-pounds. A slight consideration of the question will make it plain that the only pressure which can be multiplied by volumes in cubic feet, with the resultant product in foot-pounds, is one measured in pounds per square foot. The factor of 144 must therefore be entered into all such equations to translate pounds per square inch into pounds per square foot. 
be treated similarly to Problem I3; in fact, its $Q_{1}$ is the same. The second portion can be treated as Problem 18 . For the entire cycle the $Q_{w}$ is obviously equal to the sum of the $Q_{w}$ 's for the two portions. The $Q_{2}$, if desired, may be found from the $Q_{1}$ and the $Q_{w}$.

Problem 21. What would be the efficiency of the engine of Problem 20 if it were to exhaust into a vacuum of $25^{\prime \prime}$ instead of into the atmosphere?

The cycle now describes the path JCDURIJ.

\section{Wire-drawing. The Curve of Constant Heat}

Let $A$ and $B$, Fig. I3, represent two steam-chambers or pipes in which the pressure is maintained at $P$ and $p$ pounds per square inch respectively, connected by a passageway of relatively much smaller diameter, through which the steam finds its way from $A$ to $B$ with comparative difficulty. This passageway may be a partially opened throttle-valve or blow-off valve, or it may be a long line of pipe too small for its task, or it may be the aperture in the diafram of a Peabody throttling-calorimeter. In any such case it is only necessary, in order that the following discussion shall apply, that (I) the pressure in $B$ be less than in $A$, and (2) that the velocities in $A$ and $B$ be small in comparison with that in the passageway.

Under these conditions let $Q$, Fig. I4, represent the heat-condition of a steam-and-water mixture present in the chamber $A$. The diagram shows it to be about $3 \%$ wet; but this is only to illustrate a single application, that of the throttling-calorimeter used with commercial steam. It might be quite dry, it might be superheated, or it might be all water; the argument would apply equally well.

As the steam leaves the chamber $A$, it finds itself under a decreased pressure. As pressure is all which keeps up 
the temperature, its diminution allows the temperature to drop; it therefore starts down the adiabatic $Q d$. This process, however, even when started upon by only an infinitesimal degree, must develop mechanical energy in corresponding amount. There being no other form for this energy to take under the conditions, it takes the kinetic form; or, in other words, the elastic condition of the steam, due to its heat, sets up a flow toward the lower pressure.

The existence of flow immediately engenders friction, and friction is nothing more than a name for the transformation of kinetic energy into heat. By this double

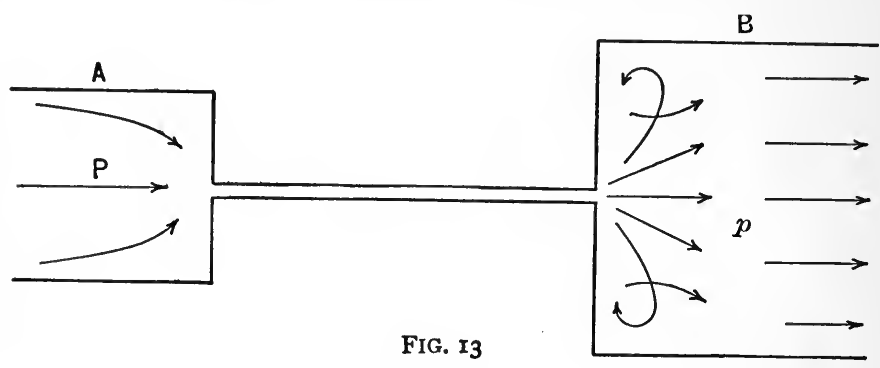

energy-transformation, therefore, the heat-energy disappearing in the first infinitesimal drop in temperature, which would be measured by the area of the rectangle of infinitesimal height running from $C C_{1}$ out to $Q$, must immediately reappear as heat which is absorbed by the steam which developed it. For no energy can be lost, either as heat or as work; all must be returned to the steam by friction, eddy, or impact.

The return of this heat, at the instantaneous pressure then prevailing, appears in the form of isothermal vaporization along the line $C_{1} Q_{1}$ to an infinitesimal amount and must be measured by the area under the process-curve. This curve is a horizontal line $d N$ in length. The area 
beneath it is the rectangle of infinitesimal width $Q_{1} d_{1} d$, the area of which is $T_{1} d N$.

By a series of such infinitesimal steps is developed the curve $Q_{1} Q_{2} Q_{3} q$. The law which defines it is that the heatenergy apparent at any point must be equal to that at any other point, because equal to that originally present at the

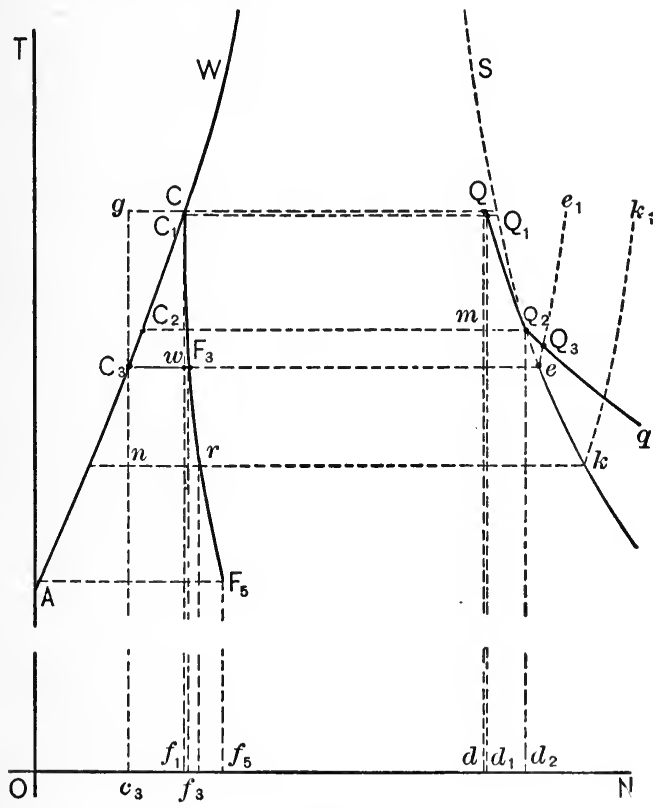

FIG. I4

point $Q$. This may be stated geometrically by saying that the area under the constant-pressure line to any point of the curve, such as the area beneath $A C_{1} Q_{1}, A C_{2} Q_{2}$, $A C_{3} e Q_{3}, A C_{4} k Q_{4}$, etc., must be equal to that at the original point; for the integration of the horizontal and vertical infinitesimal rectangles to equal limits must result in equal areas. $C_{2} C_{1} Q_{1} Q_{2}$ is therefore equal to $Q_{1} d_{1} d_{2} Q_{2}$. Subtracting the area $Q_{1} m Q_{2}$ from each side and adding the 
area, $O A C_{2} m d_{1}$ gives $O A C_{1} Q_{1} d_{1}=O A C_{2} Q_{2} d_{2}$, which is merely the graphical statement of the second sentence of this paragraph.

The heat-quantity of the point $Q_{1}$, which is also the heatquantity of every other point in the curve $Q_{1} Q_{2} q$, is called the characteristic of the curve. There are of course as many constant-heat curves on the diagram as there are different quantities of heat imaginable.

It is important to deduce the equation of the constantheat curve.

The simplest case is where the curve originates in the liquid-line $A W$, as at $C_{1}$, and develops the course $C_{1} F_{3} F_{5}$, etc. Let $F_{3}$ be any point and let the subscript 3 be omitted from the algebraic notation. From the nature of the curve, the area $c_{3} C_{3} C_{1} f_{1}$ must equal the area $c_{3} C_{3} F_{3} f_{3}$, or

$$
S\left(T_{1}-T\right)-T S \log _{e} \frac{T_{1}}{T}=T\left(N-N_{1}\right)
$$

whence

$$
N-N_{1}=S\left(\frac{T_{1}}{T}-\mathrm{I}-\log _{e} \frac{T_{1}}{T}\right)
$$

Differentiating, $\quad d N=S \frac{\mathrm{I}}{T^{2}}\left(T-T_{1}\right) d T$.

The area $f_{1} C_{1} F_{3} f_{3}$ is made up of a summation of elementary vertical strips each having the width $d N$ and the height $T$. Therefore this area may be expressed mathematically as equal to $\int_{T_{3}}^{T_{1}} T d N$. Substituting the value just found for $d N$, integrating and reducing,

$$
\begin{gathered}
\text { area } f_{1} C_{1} F_{3} f_{3}=S\left(T_{1}-T_{3}\right)-T_{1} S \log _{e} \frac{T_{1}}{T_{3}} \\
=\operatorname{area} c_{3} C_{3} C_{1} f_{1}-\text { area } c_{3} g C_{1} f_{1}=- \text { area } C_{3} g C_{1} .
\end{gathered}
$$

(The algebraic sign, in thermodynamic work, indicates merely the direction in which the heat is travelling in relation to the form of energy portrayed by the diagram or 
equation in question. In final results, therefore, it may usually be neglected.)

Stated in general terms, this result just demonstrated becomes :-

When the energy involved in any liquid isomorphic drops in temperature by wire-drawing, the area beneath the constant-heat curve and between ordinates is equal to that between the original isomorphic, the abscissa through its upper end and the ordinate through its lower end.

The Peabody Calorimeter. - In this instrument, invented by Prof. Peabody, the chamber $A$ of Fig. I 3 consists of a steam-pipe, etc., from which a sample of steam is to be drawn for the purpose of determining its moisture and heat-value. The small passageway is usually represented by a small hole in a diafram, but it may be a slightly opened valve or a length of very small, well-lagged pipe without affecting the thermodynamics of the question. The chamber $B$ is made large enough to let the steam come practically to rest and is equipped with a thermometer-well and with every possible precaution against the radiation of heat. It is best made freely open to the atmosphere, the pressure within it being determined by barometer; but it may be closed from the air by a valve, if preferred, in which case a pressuregage must be applied. The only data necessary for an observation are (I) the pressure in $A,(2)$ the pressure in $B$, and (3) the temperature in $B$ immediately opposite the steam-entrance. The first gives the level of the $C_{1} Q_{1}$-line of Fig. I4, the second the level of the $C_{3} e$-line, and the third the elevation of the point $Q_{3}$, on the line $e \epsilon^{\prime}$. Algebraically,

$$
l_{1}+y L_{1}=H_{3}+0.55\left(t_{a}-t_{3}\right),
$$

wherein the quantities in the first member apply to the observed pressure in $A$, and those of the second to the 
barometric or other pressure in $B$. The unknown proportion of steam in the steam-and-water mixture at $Q_{1}$ is represented by $y$. The observed actual temperature in the chamber $B$ is represented by $t_{a}$. The temperature of saturation corresponding to the observed pressure in $B$ is $t_{3}$. In this equation $y$ is the only unknown quantity.

Problem 22. Under a barometric pressure of 29.3", steam is drawn from a pipe under a pressure of 93 pounds by gage and shows in the calorimeter a temperature of $228^{\circ} \mathrm{F}$. What was its original percentage of moisture?

Problem 23. In the common marine practice of drawing steam from the boilers at a pressure of 300 pounds by gage and throttling at the engine to 250 pounds, how much of an original $2 \%$ of moisture in the steam as it left the boiler will reach the engine, radiation being neglected?

The Fireless Locomotive. - This type of street-railway motor was widely experimented upon about $1870-1880$ in France, and somewhat also in this country. It consisted of wheels driven by an ordinary pair of locomotive engines and carrying a plain tank fit for an internal pressure of about 300 pounds to the square inch. At the start this tank was nearly filled with water. Into the water steam was blown from a high-pressure boiler until the water was brought to the saturation-temperature of the boiler and would condense no more steam. From the top of the tank, or from a dome, steam was drawn through a reducingvalve to supply the engines. As the draft of steam reduced the pressure in the tank, the heat of the water vaporized a portion of itself into steam, and thus the engines were driven, the temperature in the tank steadily dropping until its pressure was no more than sufficient to drive the engines, when it must be recharged. The fireless locomotive never reached practical success, by reason of its weight and of the early appearance of its much more 
agile rival, the electric trolley-car. But it presents a none the less interesting problem in thermodynamics, which represents a wide class of actual situations arising wherever hot water is stored and drawn upon through a reducingvalve for a supply of steam.

Problem 24. If a fireless locomotive have a cylindrical tank 6 feet in diameter by I 5 feet long, with spherical ends of 6-foot radius, filled with water heated to a pressure of 300 pounds by gage; if the pressure be reduced to 80 pounds by gage at the engine; if it requires 40 horsepower to move the train at 15 miles per hour and the general efficiency of cylinders and connecting gear be $50 \%$; how far will the locomotive travel before recharging becomes necessary? (The exhaust is of course atmospheric.)

Referring to Fig. 14, let $C_{1}$ be the 300-pound temperature-level, let $C_{3}$ be the 8o-pound temperature-level, and let $C_{4}$ be the atmospheric-pressure temperature-level. Then the heat in each pound of water when the locomotive is freshly charged will be measured by the area $O A C_{1} f_{1}$. When the locomotive has travelled until its tank-pressure has fallen to 80 pounds by gage, each pound of this water contains the heat $O A C_{3} c_{3}$. To be sure, much of the original weight of water has left the boiler during the trip; but as is always the rule, locality does not count. Wherever the water may be, in order to get out of the boiler it must have had at least the latter amount of heat per pound.

The difference between these two heat-quantities, or the area $c_{3} C_{3} C_{1} f_{1}$, is the energy which has left the tank through the reducing-valve during the trip.

Of this energy the portion $C_{3} C_{1} w$ converts itself, by wire-drawing through the reducing-valve and the consequent isothermal vaporization, into the area $f_{1} w F_{3} f_{3}$. The total steam-heat which reaches the cylinders is, therefore, the area $c_{3} C_{3} F_{3} f_{3}$. Of this, only that above the $C_{4}$-level of 
atmospheric exhaust is available for doing work. In other words, the heat which represents the work available for drawing the locomotive is the area $n C_{3} F_{3} r$.

From the steam-table, the temperature at $C_{3}$ is 784.3 , and the heat of the liquid is 293.58. At $C_{1}$ the temperature is 882.45, and the heat of the liquid is 396.44. The area $c_{3} C_{3} C_{1} f_{1}=396.44-293.58=102.86$ B.t.u. This is also equal to the area $c_{3} C_{3} F_{3} f_{3}$. Of it only proportion $\frac{C_{3} n}{C_{3} c_{3}}$
is available for work, or $n C_{3} F_{3} r=\frac{784.3-672 . \mathrm{I}}{784.3} \cdot \mathrm{I} 02.86=\mathrm{I} 4.7 \mathrm{I} 4$ B.t.u $=\mathrm{I} \mathrm{I}, 447 \mathrm{ft} . \mathrm{lb}$.

At the start the tank contains 23,680 pounds of hot water. The efficiency of transmission is $50 \%$. To move the train 15 miles requires 40 horse-power for one hour, or 79,200,000 foot-pounds. Therefore the distance which the locomotive will travel, with the train attached, before its tank-pressure falls to 80 pounds by gage, is

$$
\frac{23,680 \times \text { I I }, 447 \times 0.50}{79,200,000} \cdot \text { I }_{5}=25.6 \text { miles. }
$$

Problem 25. When the blow-off cock of a boiler operated at 80 pounds pressure by gage is opened, what proportion by weight of the issuing current is steam?

The foregoing illustrations cover, so far as the author is aware, the ultimate limiting theory of every problem which may possibly arise in any form or variety of steam-engine or of steam-apparatus. 


\section{CHAPTER V}

\section{THE LAWS OF THE PERMANENT GASES}

All forms of matter occur in solid, liquid, or gaseous form, according to the pressure and temperature to which they are subjected. With most of them the change from liquid to gaseous is more or less gradual. As heat is added there is first evolved a form of matter called a vapor, of which ordinary steam is a good example. Upon further heating this is superheated and gradually becomes a perfect gas. The vapors obey many of the laws of the gases and are distinctly similar to them in their characteristics; but in some points they are quite different, notably in their action under heat. The gases, on the other hand, are distinguished from the vapors by no hard and fast line. They are merely highly superheated vapors.

It is to be noted most emphatically, however, that the laws controlling the mixtures of liquids and their saturated vapors bear a strong contrast to those of the highly superheated vapors called the "permanent gases." This contrast or distinction, although developed by a differentiation which is gradual, is broad and wide. It must never be lost sight of in thermodynamic practice. In the preceding chapter attention was centred upon the laws governing the effects of heat upon liquids, saturated vapors, and mixtures of liquid and vapor; in this chapter are discussed the quite different laws governing the action of heat upon the permanent gases. The laws of the heat itself, underlying both, are invariable. 
BOYLE'S LAW. - When the temperature of a gas remains constant, the pressure must vary inversely with the volume.

Expressed mathematically, this reads :-

$$
\frac{P}{P_{0}}=\frac{V_{0}}{V} .
$$

Charles's LAw. - When the volume remains constant, the pressure must vary directly with the absolute temperature. Expressed mathematically,

$$
\frac{P}{P_{0}}=\frac{T}{T_{0}},
$$

where the zero subscript refers to the original temperature.

Suppose the original condition of a gas to be the pressure $P^{\prime}$, the temperature $T^{\prime}$, and the volume $V^{\prime}$. Suppose that, while the temperature is kept constant at $T^{\prime}$, the volume is changed to $V^{\prime \prime}$. The new pressure, $p$, must equal $\frac{P^{\prime} V^{\prime}}{V^{\prime \prime}}$. Suppose, again, that, the volume remaining constant at $V^{\prime \prime}$, the temperature be altered to $T^{\prime \prime}$. The latest pressure, $P^{\prime \prime}$, must equal $p \frac{T^{\prime \prime}}{T^{\prime}}$, whence

$$
P^{\prime \prime}=\frac{P^{\prime} V^{\prime} T^{\prime \prime}}{V^{\prime \prime} T^{\prime}} \text {, or } \frac{P^{\prime} V^{\prime}}{T^{\prime}}=\frac{P^{\prime \prime} V^{\prime \prime}}{T^{\prime \prime}}=\text { a constant. }
$$

From this, if the pressure be constant,

$$
\frac{V}{V_{0}}=\frac{T}{T_{0}}
$$

The nature of the constant of Equation 25 can be seen from a consideration of the origin of the idea of the absolute zero of temperature; viz., that, since gas at $32^{\circ} \mathrm{F}$. increases $\frac{1}{493}$ of its volume for each degree of alteration in temperature, a reduction in temperature of $493^{\circ}$ must result in a reduction of the volume to zero, beyond which there could be no further abstraction of heat. Any volume of 
gas may therefore be imagined as having been derived from heating a zero volume from absolute zero of temperature to the given one. During this process the external work performed must be equal to $P V$. The external work done per degree change in temperature must be equal to $\frac{P V}{T}$. But this is the constant of Equation 25. Therefore :-

The relation of pressure, volume, and temperature of a given mass of any gas at any instant is given by the equation

$$
\frac{P V}{T}=C
$$

in which the value of the constant is the number of foot-pounds of external work done during an alteration of temperature of one degree under constant pressure.

The volume of one pound of air at $32^{\circ} \mathrm{F}$. under a pressure of 14.69 pounds per square inch is 12.39 cubic feet.

$$
\frac{\mathrm{I} 2.39 \times \mathrm{I} 4.69 \times \mathrm{I} 44}{492.8}=53 . \mathrm{I} 8 \text { foot-pounds. }
$$

Therefore it always holds true for one pound of air that its pressure in pounds per square inch times its volume in cubic feet is 53.18 times its absolute temperature in Fahrenheit degrees, or for air,

$$
P V=53.18 T \text {. }
$$

The specific volume of air is usually stated in the handbooks as a certain volume at a certain temperature under a certain pressure. This set of three figures it is impossible to retain in the memory. The single figure, 53.1 8, however, can easily be so retained. From it the volume of a known weight of air under any given conditions can be calculated, and more readily even than from the data given in the hand-books. 
A slight consideration of the method of derivation of the significance and the value of this constant will show that for other gases than air it must be inversely proportional to the density of the gas in question. From a table of specific gravities and the figure 53.18, therefore, can be readily calculated the volume of a known weight of any gas under any given conditions.

Specific Heats of Gases. - When gas is heated, one pound of it does not always absorb the same amount of heat per degree rise in temperature, independently of conditions, as is approximately the case with water, etc. If the gas be heated while the volume is kept constant, it is obvious that the only energy absorbed is that required to impart to the vibrating molecules the increased velocity which expresses itself to our senses as an increase in temperature. On the other hand, if the same gas be heated to a like degree under constant pressure, not only must this same energy be supplied in order to increase the velocity of its molecular vibration, but also its volume will increase and external work must be done in overcoming the external pressure through this increase in volume. The heat absorbed in increasing the internal, vibratory energy of the molecules is the same in either case, otherwise the temperatureincrease would not be the same. The external work of the latter case is quite additional to this and is peculiar to the condition of pressure kept constant.

If $S_{v}$ and $S_{p}$ be the specific heats under constant volume and constant pressure respectively, then, since the constant $C$ of Equation 27 is the external work done in one degree rise in temperature under constant pressure,

$$
S_{p}=S_{v}+\frac{C}{778}
$$

(since the specific heats are measured in B.t.u., and the $C$ in footpounds). 
If $K$ be the ratio between the two specific heats, then

and

$$
\begin{aligned}
K & =\frac{S_{p}}{S_{v}} . \\
S_{v} & =\frac{C}{778(K-\mathrm{I})} .
\end{aligned}
$$

A table of the values of $S_{v}, S_{p}$, and $K$ for the gases most commonly met with in engineering problems will be found in the Appendix. It will be noticed that they all lie fairly closely together, with the exception of hydrogen; and this gas, in spite of having a specific heat some fourteen times as great as the others, has a value of $K$ very similar to the rest.

Expansion-curves, $\boldsymbol{P} \boldsymbol{V}$-diagram. - When a gas expands without special means being taken to maintain either pressure or temperature constant, its pressure and volume change according to some law which usually can be expressed by the equation $P V^{x}=$ a constant, or

$$
\frac{P_{1}}{P_{2}}=\left(\frac{V_{2}}{V_{1}}\right)^{x}
$$

the latter usually being the more convenient form for use in calculation. When air or similar gases expand or are compressed in the cylinder of a motor or compressor, $x$ may assume any value between unity and about I.40.

When a gas expands in this way, the external work done is that involved in pushing back the resisting pressure $P$ through the distance represented by the increase in volume. But $P$ not being constant, the amount of external work done is not directly observable.

Let the curve $A B$ of Fig. I 5 represent the pressurevolume changes of a gas expanding or being compressed according to Equation 32. The external work done during an infinitesimal amount of expansion, at any point, as 
$c$, must be the area of the elementary rectangle $c v$, or $P d V$. The total external work done in expanding between $A$ and $B$ must be the integration of this expression, or the area $V_{2} B A V_{1}$, or

$$
\begin{aligned}
\dot{W}=\int_{V_{2}}^{V_{1}} P d V & =P_{2} V_{2}^{x} \int_{V_{2}}^{V_{1}} \frac{d V}{V^{x}}=P_{2} V_{2}^{x} \cdot \frac{V_{1}^{1-x}-V_{2}^{1-x}}{\mathrm{I}-x} \\
& =\frac{P_{2} V_{2}-P_{1} V_{1}}{x-\mathrm{I}}=\frac{C\left(T_{2}-T_{1}\right)}{x-\mathrm{I}}
\end{aligned}
$$

This is the external work actually performed in a mere change of state from the condition $P_{1} V_{1}$ to the condition $P_{2} V_{2}$.

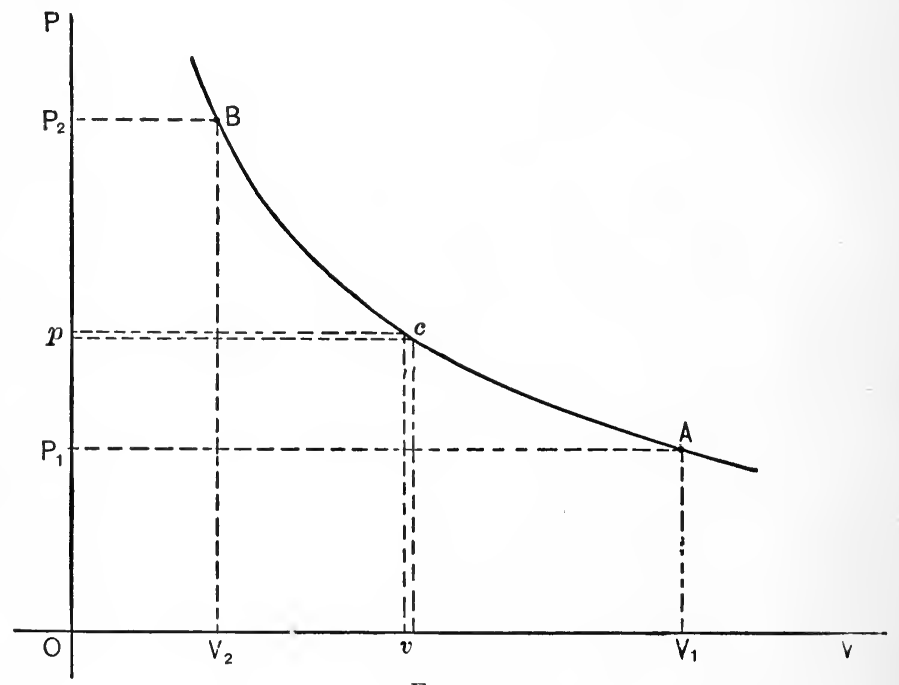

FIG. 15

In the actual expansion or compression of air or gas in the cylinder of a motor or compressor, however, each revolution of the machine involves more external work than merely that of the alteration in pressure and volume; 
there is external work involved in first drawing the gas into the cylinder before the transforming process begins, and again in afterwards discharging it from the cylinder. These quantities are in addition to that discussed above. Such a triple process is shown in Fig. I 5 by the area $P_{2} B A P_{1}$. In this the rectangle $P_{2} B V_{2}$ is the external work of taking the gas in (if the machine be a motor), and $P_{1} A V_{1}$ is that of discharging it. Of these two quantities, the former-always has the same sign as the quantity of Equation 33; the latter always has the opposite sign. Adding these two algebraically to Equation 33, or, what is the same thing, integrating the horizontal elementary rectangle $p c$, whose area is $V d p$, to the limits $P_{1}$ and $P_{2}$, there results, as the work done between abscissæ,

$$
W_{a}=\frac{P_{2} V_{2}-P_{1} V_{1}}{\frac{x-\mathrm{I}}{x}}=\frac{C\left(T_{2}-T_{1}\right)}{\frac{x-\mathrm{I}}{x}} .
$$

Equation 33 gives the true measure of transformation of heat into work during the expansion, or of work into heat during the compression, of a permanent gas; it includes no other process than the mere alteration of condition of the gas. Equation 34 gives the measure of the work actually given out by a motor, or absorbed by a compressor, in handling a perfect gas; it includes, in addition to the former process, the displacement of the gas from a locality of initial to one of final pressure.

The alteration in temperature resulting from compression from $A$ to $B$, or from expansion from $B$ to $A$, can be known by combining Equations 27 and 32 .

$$
\frac{T_{1}}{T_{2}}=\frac{P_{1} V_{1}}{P_{2} V_{2}}=\frac{V_{2}^{x} V_{1}}{V_{2} V_{1}^{x}}=\left(\frac{V_{2}}{V_{1}}\right)^{x-1}=\left(\frac{P_{1}}{P_{2}}\right)^{\frac{x-1}{x}} .
$$

It will be of mnemonic aid to note that the denominator of the expressions for the external work are $x-\mathrm{I}$ when 
referred to the volume-axis and $\frac{x-\mathrm{I}}{x}$ when referred to the pressure-axis ; and similarly, that the exponent of the expression for the alteration in temperature is $x$ - I when referred to volumes and is $\frac{x-1}{x}$ when referred to pressures.

In making arithmetical calculations of the quantities handled by these equations it will be found a great timesaver to keep them always in the form given in Equations 32 and 35 ; that is, in the form of ratios of initial and final conditions. In doing this, if the subscripts be chosen so as to make the value of the fraction greater than unity, the logarithms will always be positive and so lend themselves to the raising to odd powers with much greater convenience than if otherwise.

Equations 32 and 35 might be stated in the form;

$$
\frac{T_{1}}{T_{2}}=\left(\frac{P_{1}}{P_{2}}\right)^{\frac{x-1}{x}}, \frac{V_{2}}{V_{1}}=\left(\frac{P_{1}}{P_{2}}\right)^{\frac{1}{x}} \text {, and } \frac{P_{1}}{P_{2}}=\left(\frac{P_{1}}{P_{2}}\right)^{1} ;
$$

or Equation 25 might be stated in the form,

$$
\frac{P_{1}}{P_{2}} \div \frac{V_{2}}{V_{1}}=\frac{T_{1}}{T_{2}}
$$

From either may be deduced the very convenient rule that the logarithm of the temperature-ratio involved in a given amount of compression or expansion is always equal to the difference between the logarithm of the pressure-ratio and that of the volume-ratio, when such ratios are all kept greater than unity.

Problem 26. An air-compressor handles roo cubic feet per minute of atmospheric air at a temperature of $69^{\circ} \mathrm{F}$., compressing it to 60 pounds gage-pressure according to the equation : $P V^{1.3}=$ a constant. What is the final volume and temperature of the air and how much power is absorbed? 


$$
\begin{aligned}
& P_{1}=14.5 \\
& P_{2}=74.5
\end{aligned}
$$$$
\log P_{1}=\text { I. I6I } 368
$$

Subtracting,

Dividing by $\mathbf{I} \cdot 3$,

$$
\log P_{2}=\underline{\text { I.8721 } 56}
$$

$$
\frac{0.710788}{0.06} \frac{P_{3}}{P_{1}}
$$

$\log \frac{V_{1}}{V_{2}}=\underline{0.546760}$

Subtracting,

$$
\overline{0.164028}=\log \frac{T_{0}}{T_{1}}
$$

$$
T_{1}=66+49 \mathrm{I}=530 \quad \log T_{1}=\underline{2.724276}
$$

Adding,

$$
\overline{2.888304}=\log T_{2}
$$

$$
T_{2}=773.2 \text { absolute. } \quad t_{2}=312.2 \mathrm{~F} .
$$

$V_{1}=\mathrm{I00} \quad \log V_{1}=2.000000$

$$
\begin{aligned}
\log \frac{V_{1}}{V_{2}} & =\frac{0.546760}{\mathrm{I} .453240}=\log V_{2} \\
V_{2} & =28.39 \text { cubic feet. }
\end{aligned}
$$

The Work Absorbed $=\frac{P_{2} V_{2}-P_{1} V_{1}}{\frac{x-1}{x}}$

$$
=\frac{[(74.5 \times 28.39)-(14.5 \times 100)] \mathrm{I} 44}{0.3 \div \mathrm{I} .3}
$$

$=446,000$ foot-pounds (per minute)

$=\mathrm{I} 3.5$ horse-power.

This sample calculation includes every digit essential to the solution of the problem, except that the arithmetical computation of the last equation (for the larger numbers in which logarithms are already noted) is omitted.

Adiabatic Expansion of Gases in the $P V$-diagram. - In adiabatic expansion the external work done must be at the expense of internal energy ; there is no other source whence it may come. In the perfect gases the internal energy is all of the temperature or molecular-velocity form, and its 
specific amount per degree alteration in temperature is the specific heat at constant volume, $S_{v}$. Whence, if the gas be expanded from a temperature $T_{1}$ to $T_{2}$, the internal energy lost or gained must be

$$
S_{v}\left(T_{1}-T_{2}\right)=\frac{C\left(T_{1}-T_{2}\right)}{778(K-\mathrm{I})}
$$

But, from Equation 33, the external work done during expansion between those same temperatures is, if expressed in B.t.u.,

$$
\frac{C\left(T_{1}-T_{2}\right)}{778(x-\mathrm{I})}
$$

If the expansion be adiabatic, these two quantities must be equal, whence $x=K$. Therefore, the $P V$-equation for the adiabatic expansion of a perfect gas is

$$
\frac{P_{1}}{P_{2}}=\left(\frac{V_{2}}{V_{1}}\right)^{K}
$$

Isothermal Expansion of Gases in the $P V$-diagram. - If the expansion be isothermal instead of adiabatic, $T$ becomes a constant and Boyle's law prevails. The equation of the curve $A B$, Fig. 15 , becomes $P V=\mathrm{a}$ constant, and an analysis similar to that by which Equation 33 was derived gives as the external work done during the expansion or compression

$$
W=P_{1} V_{1} \log _{e} \frac{P_{1}}{P_{2}}=P_{2} V_{2} \log _{e} \frac{V_{2}}{V_{1}}
$$

The work done between horizontals, representing the case of the actual motor-cylinder, is in this case the same as that between verticals.

Regnault's Law. - It was announced by Regnault that the specific heat of gases, taken under constant pressure, is the same for all temperatures. This is so nearly true that it will be accepted as such for all engineering pur- 
poses. But the fact that it is not quite true shows it to be a statement of coincidence rather than a law.

Joule's Law. - It was announced by Joule that when $a$ gas is allowed to expand without doing work its temperature remains constant. This experiment was tried by placing two vessels containing air at different pressures in a tub of water until all temperatures were alike. Communication was then opened between the two. The gas in the vessel under heavier pressure expanded into the other; but no work was done, the system being considered a unit. The water revealed no alteration of temperature in the air.

The explanation of the result is that all of the internal energy of the gas consists of kinetic molecular energy, or sensible heat, the disgregation work being zero because of the wide separation of the molecules of a gas one from another and their consequent small attraction for one another. But just because their mutual attraction, according to the law of gravitation, can never be quite reduced to zero by separation, though easily made imperceptible, it is obvious that the disgregation work even of a gas must be some positive quantity; and if so it must be supplied during expansion, no matter whether external work be done or not, at the expense of the temperature-heat. Some change in temperature must result. This was proven by Lord Kelvin in some very delicate experiments upon the flow of gas through a porous plug. The fact has become of importance in the industrial arts within a very few years by the invention of the modern method for the production of liquid air, which depends entirely upon the absorption of temperature-heat in disgregation work when air expands without doing work.

That this matter may be given further consideration, let attention be turned to Fig. I4, which is reproduced here from page 107. As the curve $Q_{2} q$ departs farther and farther from 
the saturation-curve $S S$ it will plainly become more and more nearly horizontal and straight. It must be asymptotic to $O N$. At a great distance away from $S S$ it must represent the condition of a very highly superheated vapor at a very low pressure. With steam, which Fig. I4 has been drawn to represent, these conditions would be so

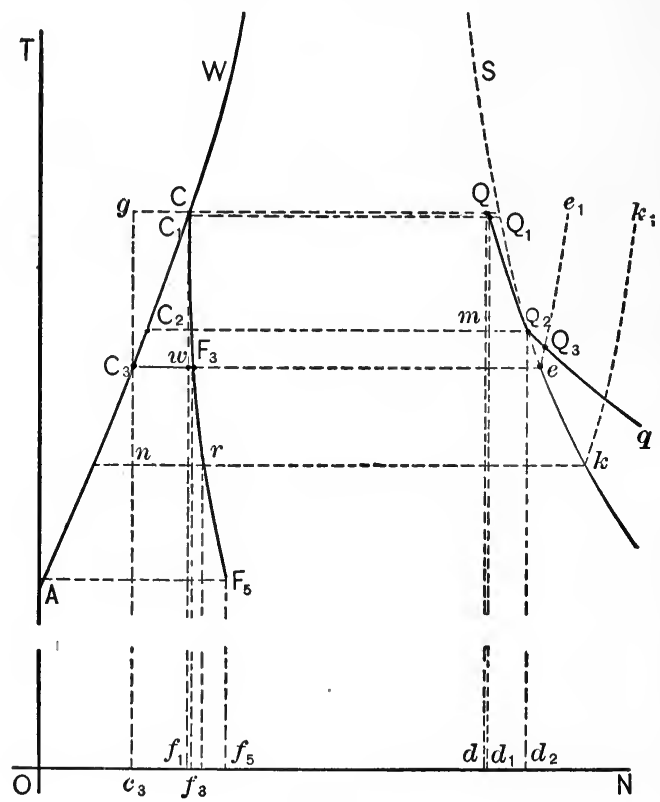

FIG. I4

extreme as never to be found in actual occurrence upon the earth's surface. But if Fig. I4 be used to illustrate the thermal condition of one of the permanent gases, or of air, with which vapor-pressures are very much higher for a given temperature, and vapor-temperatures very much lower for a given pressure, than with steam, such a distant point to the right of $S S$ might well represent the thermal energy of air under quite familiar conditions. 
If two adjacent points, both quite distant from $S S$ upon the now nearly horizontal constant-heat curve $Q_{2} q$, be considered, it is plain that transition from one to the other must involve alteration of pressure, since $Q_{2} q$ crosses all of the constant-pressure isomorphs. But drop in pressure without alteration in total fund of heat was the process with which Joule experimented in establishing his so-called law. It is now evident, from the graphical argument involved in Fig. I4, that such a process could not possibly take place without drop in temperature, because following the curve $Q_{2} q$, which is asymptotic to $O N$. But Fig. I4 at the same time reveals the reason why Joule noticed no drop in temperature; the curve $Q_{2} q$ had become so nearly horizontal that the maximum variation in pressure possible developed a drop which was at that time imperceptible.

It is now plain, in the light of accumulated knowledge, that Joule's free expansion of air was necessarily an illustration of the second law of thermodynamics. It could not have taken place, there would have been no motivepower to initiate it, had there been no concomitant drop in temperature. Drop of energy in intensity is the sole motive-power for all natural phenomena.

Liquid Air. - In Fig. I6 let the curves $W$ and $S$ represent the entropy-temperature relations of liquid air and saturated vapor of air respectively. Since the critical temperatures-of oxygen and nitrogen are $-\mathrm{I} 8 \mathrm{I}^{\circ}$ and $-23 \mathrm{I}^{\circ} \mathrm{F}$. respectively, or 280 and 230 degrees absolute Fahrenheit respectively, the entire diagram must be imagined as lying below these very low temperatures. The boiling-points under atmospheric pressure of oxygen and nitrogen are $165^{\circ}$ and $144^{\circ} \mathrm{F}$. absolute respectively. Let $C C C^{\prime}$ be the constant-pressure curve at atmospheric pressure. Let $A A$ and $B B$ be two isothermals of vaporization at pressures considerably above atmospheric; in practice they are com- 
monly 30 and io atmospheres respectively. Let $A A^{\prime}$. and $B B^{\prime}$ be isomorphs of superheated air-vapor at these same pressures respectively. The ordinary thermal condition of atmospheric air would then be represented by some such point as $C^{\prime}$.

In Fig. I 7 let $C$ be the diagram of an air-compressor taking in atmospheric air and compressing it, as nearly

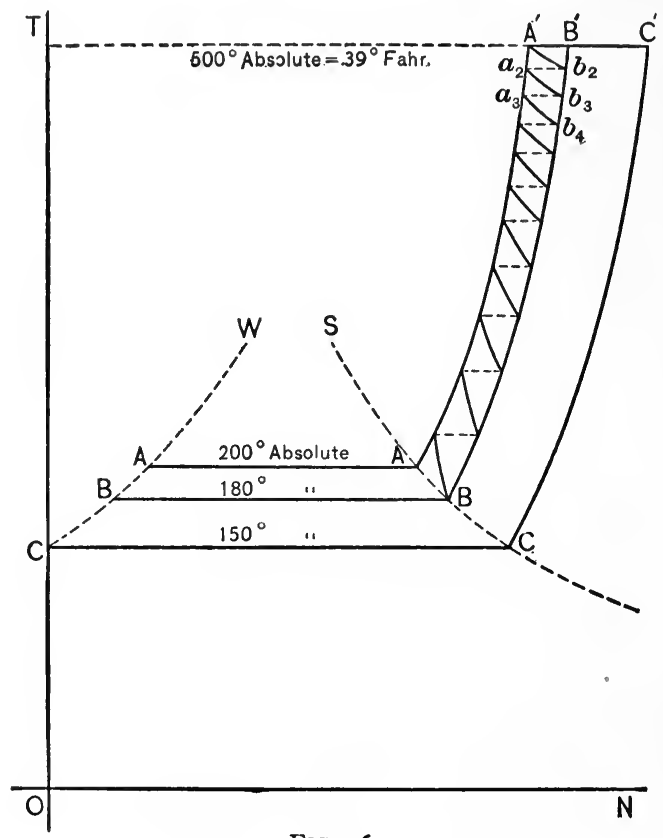

FIG. I6

isothermally as possible, to the pressure of $B B B^{\prime}$, Fig. I6, and discharging it into the reservoir $B$, Fig. I7. Let $A$ be another compressor taking its supply of air from $B$ and discharging it into the conduit $a a a$ at the pressure of $A A A^{\prime}$, Fig. I6. This high-pressure conduit aaa passes into the interior of the larger pipe $b b b$. The two together 
are formed into a coil presenting a large amount of surface and leading to an end at $V$. The coil is encased on all sides with thermal insulation $I I I$, which is constructed with great care. At the terminus of the pipe aaa is a valve $V$ opening into the larger medium-pressure pipe $b b b$ and fitted to be adjusted by hand from without. From the terminus of $b b b$ is a drain-line passing downwardly through the insulation and ending in a drain-cock $D$.

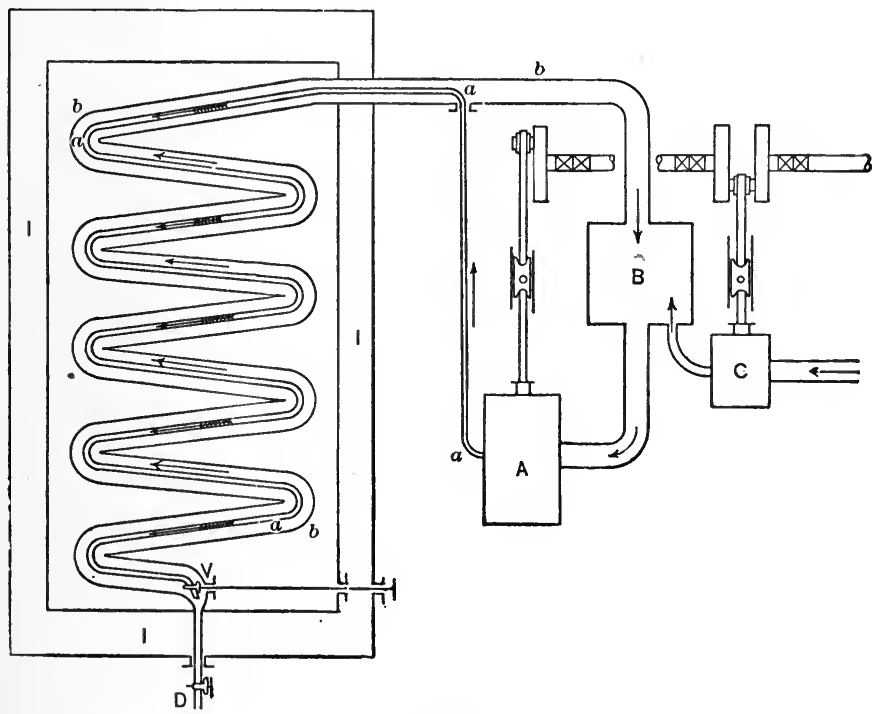

FIG. 17

The thermal activity of the apparatus illustrated in Fig. I 7 may now be traced by means of Fig. 16.

The compressor $C$ supplies air at atmospheric temperature to the reservoir $B$ at the same temperature. The compressor $A$ supplies air to the pipe aaa at the higher pressure $A A A^{\prime}$, but still at atmospheric temperature; for temperatures may be supposedly kept down to atmospheric by cold-water jackets. This double process may be shown 
in Fig. I6 by the line $C^{\prime} B^{\prime} A^{\prime}$, of isothermal compression (although properly at a higher level than drawn). The highly compressed air then traverses the pipe aaa and arrives at the valve $V$ in the condition $A^{\prime}$, Fig. I6. It finds the valve $V$ slightly open and is wire-drawn through it into $b b b$, its pressure falling to $B B B^{\prime}$ and its temperature to $b_{2}$, Fig. 16 , by passage down the constant-heat curve $A^{\prime} b_{2}$.

In traversing the pipe $b b b$, Fig. I7, this current of air brings the $A$-pressure air coming in along aaa down to its own temperature, so that the next quantity of the latter to arrive at $V$ finds itself there in the thermal condition $a_{2}$, Fig. 16. Its wire-drawing through the valve $V$ must then be represented by the constant-heat curve $a_{2} b_{3}$. The $B$-pressure air then traversing the pipe $b b b$ at the temperature $b_{3}$ necessarily brings the next incoming air to the temperature $a_{3}$, and its wire-drawing in turn develops $B$-pressure air of the condition $b_{4}$.

Lord Kelvin's formula for the drop in temperature $D$ involved in each of these passages across from the $A A^{\prime}$ isomorph to the $B B^{\prime}$-isomorph is

$$
D=0.497\left(P_{A}-P_{B}\right)\left(\frac{493}{T}\right)^{2},
$$

wherein the pressures are measured in atmospheres and the temperatures in degrees Fahrenheit. $T$ is the initial absolute temperature at the departure from the upper pressure.

So the process goes on, all temperatures within III falling steadily meanwhile, until finally the steady fall of the point successively represented by $b_{2}, b_{3}, b_{4}$, etc., results in its coincidence with $B$. As the processes which resulted in temperature-fall due to abstraction of heat still continue, their further action must result in the condensation of some of the air at the $B B$-pressure. This condensation drains 
to the valve $D$, and may be there drawn off into an external vessel.

It will be obvious, from a slight consideration of the geometrical laws controlling the curves $S B$ and $a b$, that at these very low temperatures they must be much nearer to parallelism than is the case at ordinary steam-temperatures. How nearly they approach parallelism, whether $a$ reaches $A$ before $b$ reaches $B$, or the opposite, cannot now be said. If $a$ does reach $A$ before $b$ reaches $B$, it must indicate that liquid is first formed in the pipe aaa, just above the valve $V$, before it collects at $D$. If so, this liquid is vaporized by wire-drawing through $V$ just as the moisture in a throttling-calorimeter is evaporated by a similar process. But whether this be the case, or not does not affect the thermodynamic explanation of the final result.

Carnot's Cycle with Air as a Working-Substance. Carnot's cycle, consisting as it does of two isothermals and two adiabatics, must always appear as a rectangle upon the $N T$-diagram. But the form of its $P V$-diagram depends entirely upon the working-substance which serves as a carrier for the heat. When steam performed this office it appeared that the $P V$-diagram was compounded of two horizontal rectilinear isotherms and two hyperbolic adiabatics, the area enclosed being quite considerable. Indeed, the actual steam-engine, whose success has always been founded chiefly upon its large amount of power developed per unit-weight and unit-volume of working-substance, works a close approach to the Carnot cycle. But if air or any other permanent gas be the working-substance, the isotherms and adiabatics are both hyperbolic in form and are very nearly parallel. The $P V$-diagram enclosed between any intersecting four of them, as shown in Fig. I8, is very long and narrow in proportion to its area. The range of both pressure and volume per unit of work devel- 
oped is enormous. Entirely aside from the obstacles of bulk and heating-surface which have opposed the success of all air-engines, this additional one of wide range of pressure and volume per unit of work has always prohibited all hope of seeing a successful Carnot-cycle air-engine.

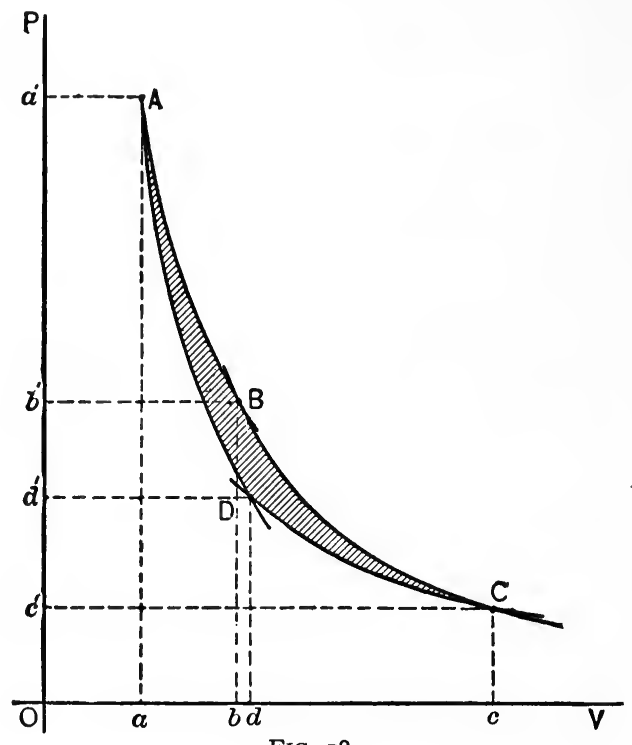

FIG. 18.

The obstacle to efficiency in the steam-engine is not the poor form of its cycle, but is the narrow practicable range in temperature. It already begins to appear that the obstacle to efficiency in the use of the permanent gases as working-substances in heat-engines is not any limitation in temperature-range, but in the poor form of their practicable cycles. This contrast will appear more strongly in the following chapters. 


\section{CHAPTER VI}

\section{THE GAS-ENGINE CYCLES}

THE history of the public distribution of artificial illuminating-gas opened with the century which has recently come to its close. Murdoch, Watt's famous assistant, had an active hand in the matter, and the shops of Boulton \& Watt were amongst the first buildings upon which the experiment was tried. The presence of the new fuel very quickly led to attempts at its utilization for the development of power. Patents for gas-engines begin to appear upon the records before 1825 . The second quarter of the century is thickly sprinkled with them. It was not until I 860, however, that an engine was produced which was sufficiently successful in a mechanical sense to attain a commercial basis, and for a long time thereafter the price of gas and the fuel-consumption of the engines both remained so high that very little progress was made in their adoption as a standard prime mover, even in the small sizes to which they were then restricted. The present position of the internal-combustion engine is due more to Dowson's invention of a cheap power-gas generator, to the natural-gas supply of the United States, to the utilization of the waste-gases from blast-furnaces for power-development, and to the wide and cheap supply of the lighter petroleum distillates, than it is to the development either of illuminating-gas or of the engine itself.

The Lenoir Cycle. - The first gas-engine sufficiently successful to command a market was the Lenoir, brought 
out by a Frenchman of that name in 1860 . The Lenoir engine much more closely resembled a single-cylinder horizontal steam-engine of the throttle-governed type than has any gas-engine since then. It was double-acting and controlled by a fly-ball governor. There the similarity ended, however. Its cylinder was water-jacketed, the valves were quite different, and the boiler was absent.

The cycle of processes in the Lenoir engine was as follows :

(I) From dead-centre the fly-wheel drew the piston out, sucking in as it did so a charge of gas and atmospheric air in the correct proportion to form an explosive mixture;

(2) Somewhat before half-stroke was reached the inletvalve was closed and the charge was ignited, producing an explosion which elevated the pressure in the cylinder considerably above atmospheric;

(3) The remainder of the stroke was devoted to expanding this pressure to atmospheric, or nearly so ; and

(4) The return-stroke exhausted the burnt gases. Thus there was produced an impulse upon each side of the piston at each stroke, or two for each revolution within a single cylinder.

The objections which led to the abandonment of the Lenoir engine were $(a)$ the great consumption of fuel, (b) the violent overheating of parts, especially the piston, and their consequent rapid deterioration, and $(c)$ the slight power developed.

Hugon was the only other builder of a true Lenoir engine, but the thermodynamic cycle survived in the freepiston engines of Barsanti $\mathcal{E}$ Matteucci and of Otto $\mathcal{E}$ Langen, who tried to improve Lenoir's fuel-consumption on the theory that it was the rapid loss of heat to the jacketed walls immediately after explosion which was the source of trouble. They therefore built vertical engines 
each with a long cylinder in which a heavy piston was driven rapidly upward by the explosion; but the piston instead of being attached to a crank and limited in speed by its motion was free to rise to the top of the cylinder at the maximum velocity possible. It carried with it a rack meshing with a pinion which rotated loosely on the overhead fly-wheel shaft. The shaft normally revolved in the direction opposite to that of the pinion during the upward stroke of the piston. When the force of the explosion and of inertia had been overcome by gravity, the piston descended, aided by the partial vacuum formed beneath it by the cooling of the spent charge, and the motion in this direction engaged the pinion with the shaft by a pawl-andratchet device. It was on this return-stroke that power was imparted to the shaft.

These free-piston engines were better than the Lenoir as to fuel-consumption; they also remained cooler and worked more reliably. On the other hand they were fearfully noisy, too noisy for toleration, and in their turn they have passed away.

The only surviving representative of the Lenoir cycle is the Bischoff, a very small vertical engine whose sole recommendation is simplicity.

Problem 27. What are the conditions of operation and the efficiency of a Lenoir engine using at each stroke one cubic foot of an explosive mixture consisting of nine-tenths air and one-tenth gas of a calorific power of 650 B.t.u. per cubic foot, the atmospheric pressure and temperature being I4. 5 pounds and $59^{\circ} \mathrm{F}$. respectively?

The first operation, that of drawing in the charge, is shown at $A B$, Fig. 22. Since this is not a thermodynamic process, but merely a change of location, it does not appear in the $N T$-diagram. Hence, $V_{B}=\mathrm{I} .00, P_{B}=\mathrm{I} 4.5$ and $T_{B}=520$. 
As to the entropy, since we are no longer relying upon the steam-tables, we need no longer adhere to their arbitrary zero of $32^{\circ} \mathrm{F}$. It will be preferable to assume an arbitrary zero-axis for entropy which passes through the initial point of this problem, $B$. Hence, $N_{B}=$ o.

Although the piston is really in quite rapid motion at $B$, and although an appreciable amount of time is required

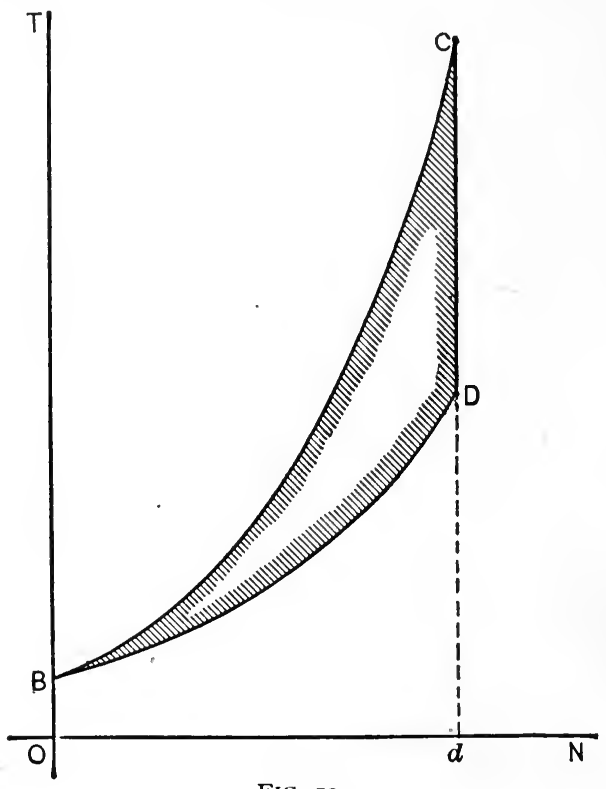

FIG. I9

for combustion to take place, yet theoretically the explosion is instantaneous and the piston accomplishes no motion while it is taking place. The next process therefore consists of the development of heat within the working-substance at constant volume. This process may be regarded as an addition of heat from without, for the heat is just as truly added to the substance when coming from its own 
chemical alteration as when coming from outside. The process is therefore properly represented by the curve $B C$, Fig. I9, whose equation is

$$
N-N_{B}=S_{V} \log _{e} \frac{T}{T_{B}}
$$

and by the line $B C$ of Fig. 20, whose equation is

$$
V=\mathrm{a} \text { constant }=V_{B} \text {. }
$$

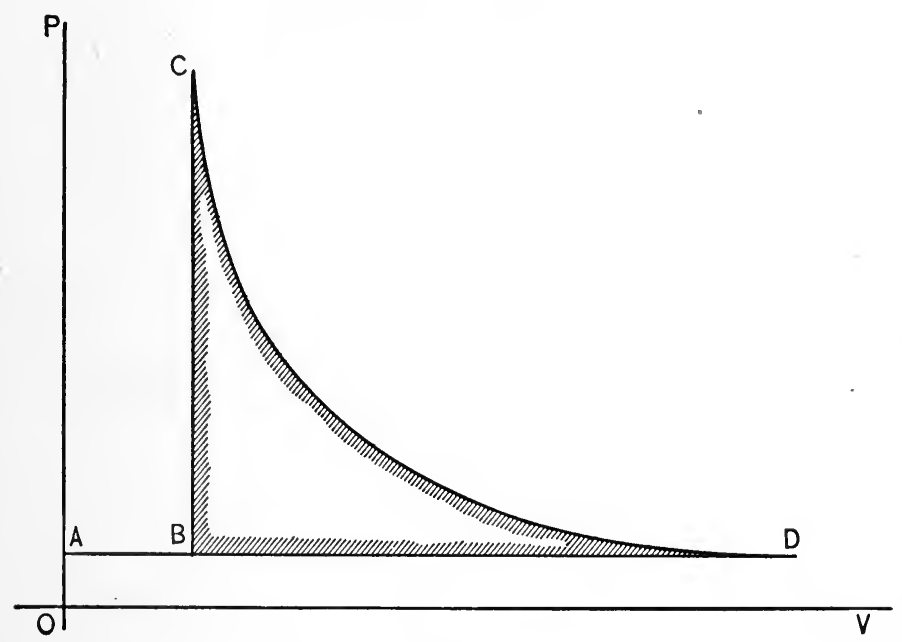

FIG. 20

Moreover, the volume being constant,

$$
\frac{P_{C}}{P_{B}}=\frac{T_{C}}{T_{B}}
$$

The available heat per unit of mass, measured by the area $O B C d$, Fig. 19, is $S_{V}\left(T_{C}-T_{B}\right)$. The mass present, $M$, may be found from the equation

$$
P V=C T \cdot M \text {, or } 14.5 \times \mathrm{I} 44 \times \mathrm{I}=53.18 \times 520 \times M,
$$


whence $M=0.0758 .^{1} \quad$ But since one-tenth of the cubic foot consists of gas having a density which may be assumed to be 0.6 , the true mass is $0.96 \times 0.0758=0.0727$. The available heat is 65 B.t.u. From these equations are derived

$$
\begin{aligned}
T_{C}-T_{B} & =5315, \\
T_{C} & =5835, \\
N_{C} & =0.02044, \\
P_{C} & =162.7 .
\end{aligned}
$$

and

The process $C D$ must be an adiabatic, unless artificially distorted therefrom. Therefore in Fig. I9 the equation of $C D$ is

and in Fig. 20

$$
N=0.02044
$$

$$
P V^{1.408}=\text { a constant. }
$$

From Equation 35 is derived the value of $T_{D}$ as 2915.

In Fig. I9 the areas beneath the curves give the heat handled, and in either diagram the enclosed area gives the work done. $Q_{1}$ is known to be 65 B.t.u.; $Q_{2}$, or the area $O B D d,=M S_{p}\left(T_{D}-T_{B}\right)=4 \mathrm{I} .3$ B.t.u. Hence $Q_{W}=Q_{1}$ $-Q_{2}=\mathrm{I} 8.7$ B.t.u. and the efficiency is $28.8 \%$.

It is needless to say that no such temperatures or efficiencies were ever attained in the actual engine; the walls absorbed too much heat.

The Beau de Rochas Cycle and the Otto Engine. - At about the time when Otto \& Langen were experimenting with their free-piston engine, M. Beau de Rochas enunciated the principles upon which a gas-engine might be

${ }^{1}$ In the majority of cases, however, the accuracy of conclusions will not be affected if the assumption be made, for convenience, that $M=$ unity, the quantity of heat being calculated per unit of mass instead of per unit of volume. 
operated successfully and economically. A few years later, in 1876 , Dr. Otto brought out his "Silent" engine embodying them. The name was designed to signify that the objectionable noise of his earlier engine was no longer present.

This cycle, usually but erroneously referred to as the Otto cycle, consisted of the following processes:-

(I) The drawing into the cylinder throughout an entire stroke of a charge of explosive mixture at atmospheric pressure.

(2) The return of the piston under the influence of the fly-wheel, compressing this charge into the clearance space, which is much larger than in steam-engines.

(3) The ignition and explosion of the charge while the piston is at rest on dead-centre, the process being now truly under constant volume.

(4) The expansion of the heated gases throughout the entire stroke.

(5) The exhaust of the burnt gases during the backstroke.

The Otto Silent engine had a great and immediate success. The bulk of the gas-engines built since $\mathrm{r} 876$ have closely followed the principles of mechanical design which it embodied, as well as its thermodynamic cycle. It has nearly always been built in single-cylinder, single-acting form, in order to keep one side of the piston open to the air and thus avoid the overheating of the Lenoir. In consequence, it gets but one impulse every four strokes, or every two complete revolutions. Although at first but slightly better in fuel-consumption than the free-piston engine, owing to too little compression, it was quiet, powerful, and reliable in operation, and in every way an improvement over what had preceded. It is now being built in two-, three-, and four-cylinder form, in order to multiply 
impulses. One or two builders have produced doubleacting engines. For many years its size was limited to an average of less than five horse-power, partly by reason of the cost of fuel and the consequently limited field of industry in which it was profitable, and partly by reason of mechanical obstacles which multiplied rapidly with increase in size. To-day the natural-gas supply of the Mississippi Valley, and the use of the waste gases from the blast-furnace for driving gas-engines in Continental Europe have raised the practicable limit in size of a single engine to 4000 horse-power, with no reason apparent why further progress in capacity may not be made.

The Clerk-cycle or "Two-cycle" Gas-engine. - The obvious mechanical objections arising from the limitation of the standard form of the Otto gas-engine to one impulse for each two revolutions led to its modification by Mr. Clerk so as to obtain an impulse once each revolution. To accomplish this a smaller preliminary or metering cylinder was added to the engine. Its office was to draw in the explosive mixture of atmospheric air and gas to the amount required. Its piston was so timed in its motion that it had partially accomplished its return-stroke, slightly compressing the contained charge, when the main piston arrived at deadcentre after the completion of its expansion-stroke. Communication between the two cylinders was then opened, allowing the slightly compressed fresh charge to enter the main cylinder, driving before it the burnt gases out the exhaust-valve. At the moment when the fresh charge had practically displaced the burnt one both exhaust- and admission-valves were closed, and the return of the main piston prepared the fresh charge for ignition without the mediation of a pair of exhaust- and suction-strokes.

As built now, chiefly for marine and locomobile work, or in the smaller sizes for stationary work, the "two-cycle" 
engine is nearly always a single-acting vertical engine, with the connecting-rod and crank working in an enclosed crankcase. This crank-case is employed for the preliminary metering of the charge, instead of a separate cylinder. As the piston rises, the fresh charge is drawn into the crankcase, below the piston. At upper dead-centre the admission-valve is closed and the downward stroke slightly compresses the charge. At lower dead-centre communication opens between the crank-case and the cylinder, and the fresh charge expands into the cylinder, displacing the burnt charge. The upward stroke of the piston then performs the usual compression of the charge preliminary to ignition.

The builders and buyers of gas-engines draw considerable distinction between the so-called "Otto-cycle" and "twocycle" engines. It is true that, although much skill has been expended in markedly improving the two-cycle type, yet there will always be an imperfection in its action and efficiency due to the impossibility of displacing instantaneously the burnt charge by the fresh one without either losing some of the latter through the exhaust-valve or retaining some of the former within the cylinder. ${ }^{1}$ It is therefore proper to draw a distinction between the two. Yet it is obvious that the difference is mechanical, not thermodynamic, in character. The preliminary "compression" is merely a means of displacing the burnt charge. Since the exhaust-valve is wide open at the instant that

${ }^{1}$ In the large Körting engines, in the region of 1000 horse-power each, which operate upon this cycle, this difficulty is obviated by having two displacer-cylinders. One of them pumps fresh air through the main cylinder immediately after exhaust, cleansing or "scavenging" the cylinder of all burnt gases. The other follows with the fresh charge of explosive mixture, small enough in volume to ensure that none of it shall pass out through the exhaustport, and rich enough to stand dilution with the remnant of fresh air remaining after the scavenging. 
the charge enters the main cylinder, the pressure must be atmospheric. Whatever preliminary compression there was has been lost. Thermodynamically, the Otto-cycle and the two-cycle engines operate upon exactly the same cycle.

Problem 28. What are the conditions of operation and the efficiency of an Otto engine working under the same

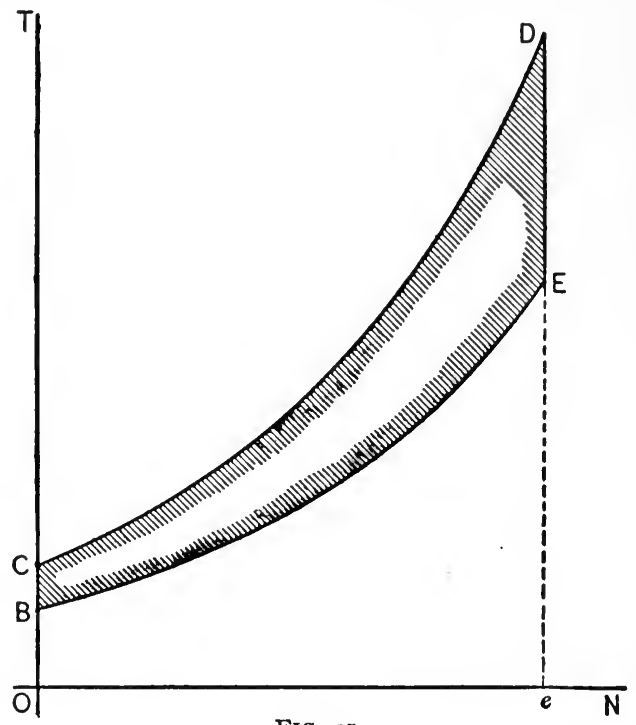

FIG. 2I

premises as the Lenoir of Problem 27, except that the charge is compressed to an absolute pressure of 75 pounds per square inch before explosion?

The cycle is depicted in Figs. $2 \mathrm{I}$ and 22. $A B$ is the suction of the charge, not appearing in the $N T$-diagram. $B C$ is the adiabatic compression, $C D$ is the explosion, $D E$ the adiabatic expansion, and $E B$ the exhaust at constant volume at the end of the stroke. The starting-point of the calculation of the cycle may now be taken as based 
upon the fact that $V_{A B}=\mathrm{I}$ cubic foot. If $P_{C}$ is to be 75 pounds per square inch, then

$$
\begin{gathered}
\left(\frac{V_{B}}{V_{C}}\right)^{1.408}=\frac{P_{C}}{P_{B}}=\frac{75}{\mathrm{I} 4.5} . \\
V_{B}=1+V_{C} . \quad \text { Therefore, } \\
V_{C}=V_{A}=0.452 \text { and } V_{B}=\mathrm{r} .452 . \\
T_{C}=837 . \quad N_{B}=N_{C}=O .
\end{gathered}
$$

To the mass of the I cubic foot of explosive mixture of Problem 27 must now be added the 0.452 cubic foot of burnt

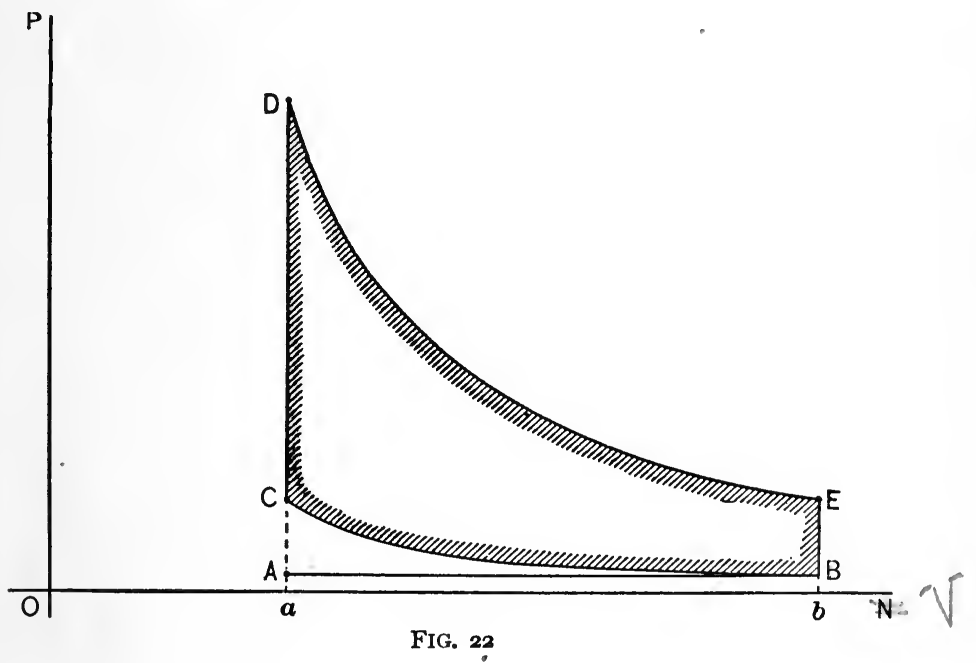

gases in the clearance-space, or $M=0.1057$. Proceeding as before, $T_{D}=4498, N_{D}=0.02972$, and $P_{D}=403$. The expansion $D E$ cannot be carried down to atmospheric pressure, because the engine's cycle limits the final volume after expansion to the initial volume before compression. ${ }^{1}$

1 See page 287 , Part II, et seq. 

Hence, $V_{E}=V_{B}=1.452 . \quad \frac{P_{D}}{P_{E}}=\frac{P_{C}}{P_{B}} . \quad P_{E}=78$, and
$T_{E}=2792$.

The two isomorphic curves $C D$ and $B E$ of Fig. $2 \mathrm{I}$ have the same equation, except that the coefficients of specific heat differ; the curves $D E$ and $C B$ of Fig. 22 bear a similar relation. $Q_{1}$ is the area $O C D e$ (Fig. 2I), and $Q_{2}$ is the area $O B E e$. But geometrical inspection will prove that these areas must bear the same proportion to one another as $T_{C}$ to $T_{B}$, or as $T_{D}$ to $T_{E}{ }^{1} \quad$ Therefore $Q_{w}=Q_{1}-Q_{2}$ must bear the same proportion to $Q_{1}$ or $Q_{2}$ as $T_{C}-T_{B}$ does to $T_{C}$ or $T_{B}$, respectively;

or

$$
\frac{Q_{w}}{Q_{1}}=\frac{T_{C}-T_{B}}{T_{C}}=\frac{T_{D}-T_{E}}{T_{D}},
$$

from which the efficiency of the cycle is found to be $37.9 \%$.

It is obvious from this that it is the vertical separation of the curves $C D$ and $B E$ of Fig. $2 \mathrm{I}$, or the proportion of the total available fall in temperature which is utilized, which determines both the efficiency and the power of the engine. This explains why the early Otto engines, with their slight degree of compression, were so slightly more economical than the Lenoir. It also explains why the chief line of progress in gas-engine design between the years 1875 and 1895 has consisted in increasing the degree of compression. Progress in this direction has now almost

${ }^{1}$ The area beneath $C D$, Fig. 2I, is equal to an integration of the expression $T d N$. The area beneath $E F$ is measured by the same expression. For any given value of $d N$ or of $N$, however, the value of these two expressions will be the proportional to the values of $T$. Since the equation of $C D$ is $N-N_{0}$ $=S \log _{e} \frac{T}{T_{C}}$ and that of $E B$ is $N-N_{0}=S_{v} \log _{e} \frac{T}{T_{B}}$, for any given value of $N$ the corresponding values of $T$ for the two curves must be proportional to $\frac{T_{C}}{T_{B}}$. Hence, the areas beneath $C D$ and $E F$, between any given pair of ordi-

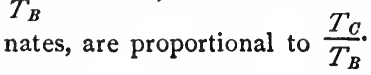


ceased, (I) because of the rapidly increasing mechanical objections due to the development of such high momentary pressures at the point $D$ without proportionate increase in work done: the engine must be strong enough, in castings and bearings, to stand this maximum pressure, and yet, because the pressure is so evanescent, the engine derives little good from it ; (2) because of the danger of preignition when the degree of compression is high, particularly in oil-engines.

The Joule Cycle. - The Joule cycle was proposed by him who discovered the mechanical equivalent of heat. It has found its embodiment in several designs of engine, none of which have survived the test of competition with the Otto type. Some of them have been gas-engines, as have the Gardie and the Bergher, some oil-engines, as the Priestman, and some almost purely hot-air engines, as the Cayley-Buckett. The Joule type is nevertheless of the greatest importance because, while no one has ever yet put it into profitable practice, it offers possibilities of superiority over the Otto which have attracted to it a long line of inventors. Their failures may in each case be easily explained as inevitable under the mistaken line of design which they adopted. It is the personal opinion of the author that the large gas-engine of the future will operate on no other than the Joule cycle. His reasons, however, are not solely, nor even largely, based upon those thermodynamic points of superiority which are developed in the following pages. They are based chiefly upon purely mechanical considerations which lie outside the limits of the present discussion. It is worthy of note, however, that the purely thermodynamic aspect of the question shows that under any chosen limits of temperature and pressure, the theoretic efficiency of the Joule cycle is not only greater than that of the Beau de Rochas cycle, but it is greater than that of any other cycles known 
to have been proposed or put into practice except the Carnot, the Stirling, and the Ericsson. Of these three, all are known by long trial to be impracticable.

The essential features of an engine operating on this cycle are shown in Fig. 23. They comprise two cylinders, $C$ and $W$, the latter larger than the other, the two being combined in any way so that power may be transmitted from one to the other; a source of heat at a higher temperature $T_{1}$; a refrigerator at a lower one $T_{2}$. Supposing

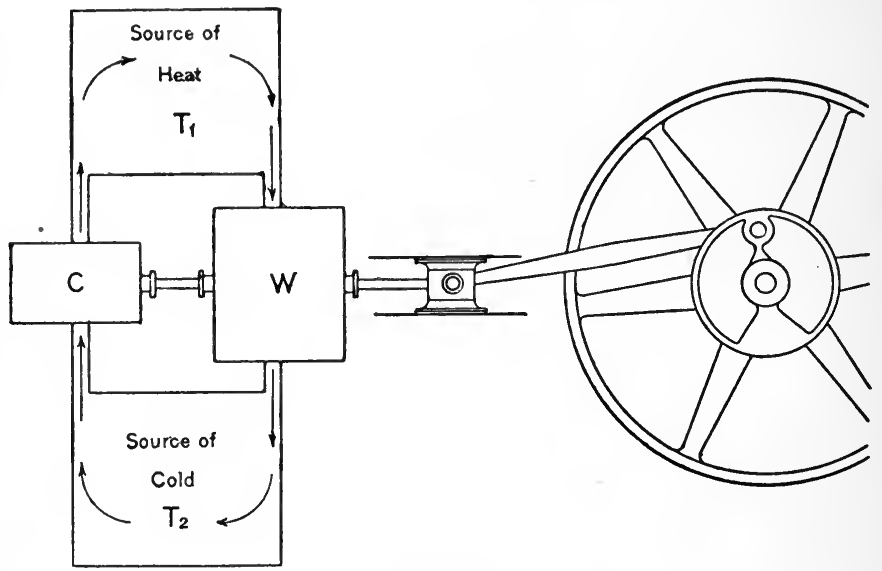

FIG. 23

the entire system to be filled with the chosen permanent-gas working-substance, air for example, the operation is as follows, the processes being traced in the diagrams of Figs. 24 and $25:-$

( I) There is drawn into cylinder $C$ a charge of workingsubstance. This is shown by $A B$ of Fig. 25 ; it does not appear in Fig. 24.

(2) The adiabatic compression of the charge to the maximum working-pressure of the engine. This is shown by $B C$ in both diagrams. 
(3) The addition of heat under constant pressure; $C D$ in both diagrams.

(This process really involves the triple process of $(a)$ the expulsion of the compressed charge from the cylinder into the chamber $T_{1}$, shown by $C c$ of Fig. $25 ;(b)$ its increase of volume while there; $(c)$ the taking of the increased volume into the cylinder $W$, which is shown by $c D$ of Fig. 25 . But as this combination consists entirely of changes of locality, it need not be expressed upon the thermodynamic diagrams.)

(4) The adiabatic expansion of the heated gases to atmospheric pressure. This is shown by $D E$ in both diagrams.

(5) Their exhaust at constant (atmospheric) pressure. This is shown by $E B$ in both diagrams.

It is immediately obvious that the process of heating the gases in the chamber $T_{1}$ may be done either by the transmission of heat to them through the surrounding walls or by their own internal combustion. If the latter be the case, the combustion must take on the characteristics of a flame, increasing in volume under constant pressure, rather than those of an explosion, which implies an increase of pressure under constant volume. In the Cayley-Buckett engine this chamber was a furnace enclosing an anthracite fire; the furnace was ordinary in every respect except that it was capable of withstanding internal pressure. In the Priestman oil-engine the working-substance was a mixture of oil and air; this chamber $T_{1}$ was a little combustion-chamber fit to contain an oil-flame and separated from the cylinder $C$ by a shield of wire gauze to prevent the flame from travelling back into the compressed charge which was being driven out of $C$. In the Gardie and Bergher engines the combustion takes place in the cylinder $W$ as the compressed charge enters. In all 
except the first the combustion is intermittent, ignition being provided every time that the cycle reaches the point $C$ and the flame being extinguished at the point $D$; in the first the flame burns more or less continuously, the chamber $T_{1}$ being sufficiently large to store the extra energy evolved during the time when the cylinder $W$ is not taking its charge.

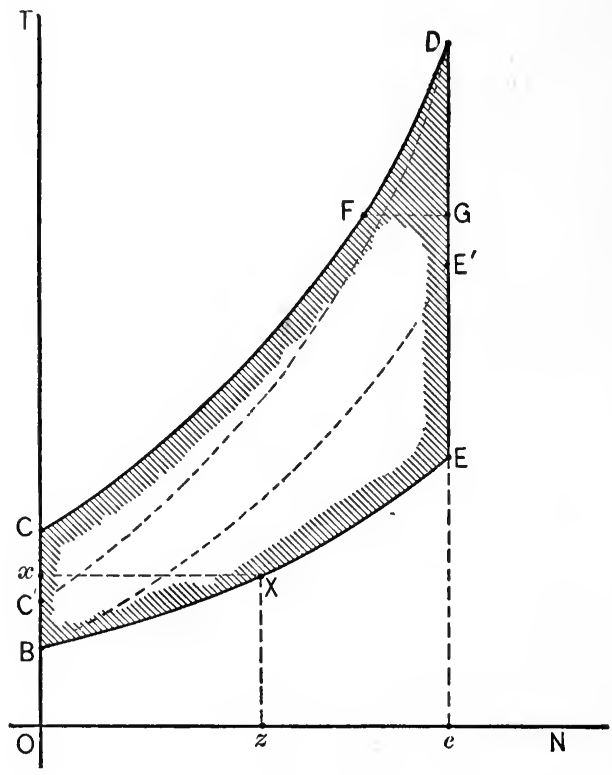

FIG. 24

Problem 29. What are the conditions of operation and the efficiency of a Joule engine working under the same premises as the engines of the two preceding problems, except that the charge is compressed to a pressure of 365 pounds per square inch absolute before ignition?

Here the original cubic foot of explosive mixture appears 
as the volume $A B$. Calculating the values for the several points as before, it appears that $V_{C}=$ O.IOI I, $T_{C}=1323$, $V_{D}=0.3995, T_{D}=5095, N_{D}=0.02438, T_{E}=2002$, and $V_{E}=3: 844$. As in the case of the Otto cycle, the curves $C D$ and $B E$ of Fig. 24 have the same equation and differ only in the point of origin; therefore the area beneath each curve is proportional to the ordinates at any abscissa, or $Q_{w}: Q_{1}$ as area $B C D E$ : area $O C D e=B C: O C=$ $803: 1323=60.7 \%$ efficiency.

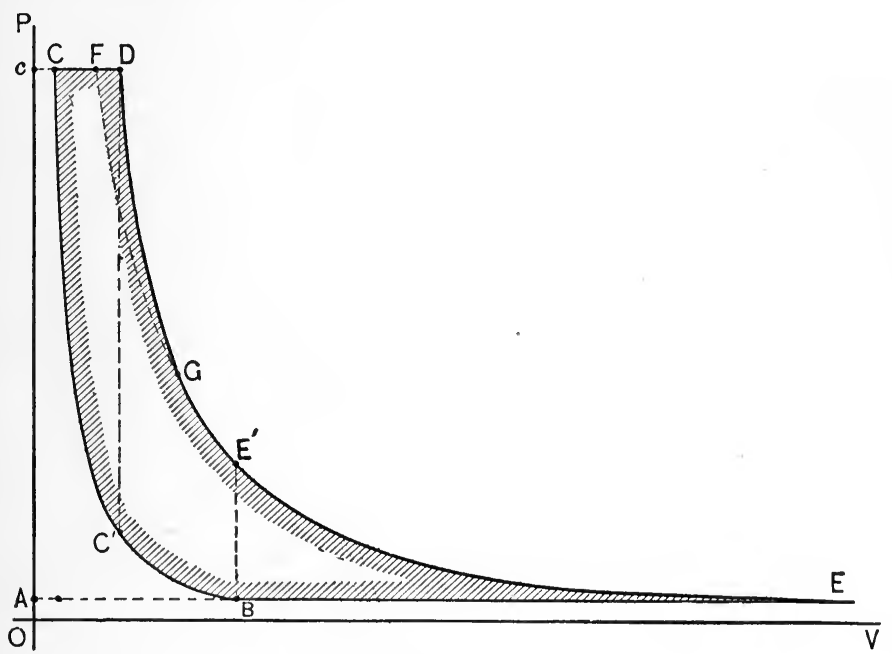

FIG. 25

Although it is obvious from these figures that the efficiency of the Joule cycle is much superior to either the Lenoir or the Otto under the conditions chosen, it is not equally clear that it would be under any conditions. The diagrams are not drawn to the same scale nor, even if so, could any set of conditions be chosen which would be equally fair for all three. But an excellent general comparison may be had by adding to Figs. 24 and 25 the 
constant-volume lines $C^{\prime} D$ and $B E^{\prime}$. Then between the one pair of adiabatics, $B C$ and $D E$, are shown:

(I) A Lenoir cycle, $B E^{\prime} E$;

(2) An Otto cycle, $B C^{\prime} D E^{\prime}$; and

(3) A Joule cycle, $B C D E$. The comparison is not fair to the Lenoir cycle, because the efficiency of the latter increases with its width; hence, in the Lenoir, the richer the gas or the oil used, the better will be the efficiency. But with both the Otto and the Joule cycles the efficiency is independent of their width in the NT-diagram, being solely dependent upon their height, or upon the degree of compression. Therefore, their limitation between a given pair of adiabatics is equally fair to both. Since the Lenoir cycle is out of the race in any event, the injustice done to it may be overlooked.

It is further obvious that the territories of the Lenoir and of the Otto cycles are mutually safe from trespass. The Lenoir type cannot get into the territory of the Otto, to the left or above $B E^{\prime}$; for it may not embody initial compression. The Otto cannot cross the same line in the opposite direction, into the territory of the Lenoir, without final expansion to a volume greater than its initial one, which is impossible.

The avoidance of this inherent limitation of the Otto cycle has been sought, in a single-cylinder type, by Mr. Atkinson in his "Differential" and "Cycle" engines, and by various designers in the multiple-cylinder type by adding larger low-pressure cylinders in which the expansion $E^{\prime} E$ might be performed; but all have failed, the first for mechanical and the second for thermal reasons.

It is also sought in some of the largest and best engines of the present day, without added mechanism, by restricting the volume taken in at atmospheric pressure to less than the full cylinder-volume. This also fails to attain 
the desired end, for reasons which are explained on page 287 .

So, with this limitation accepted as inevitable, and remembering that the chief limitations in constructive practicability are pressure and temperature, the broad conclusion may be safely drawn that under the same maximum limits of pressure and temperature, the Joule cycle has inevitably the greatest efficiency of the three, its area not only completely covering the Otto and the bulk of the Lenoir, but having some territory of its own unattainable by either of the others.

The universal failure of the Joule-cycle engines in the past can be explained, in the face of this statement, by the fact that in no case was a working-pressure adopted which would permit any possibility of success. Always arbitrarily limited by their designers to about 100 pounds as their maximum pressure, the Joule-cycle engines hitherto constructed could have no hope of successful competition with the Otto-cycle engines, when the latter regularly attained pressures of from 300 to 400 pounds as maxima.

The Diesel Cycle. - In the year I 897 Mr. Rudolph Diesel announced to the world the successful operation of an engine operating under a cycle which he had previously advocated from purely thermodynamic considerations. ${ }^{1}$ In the argument which led him to his result Mr. Diesel started in search of the nearest possible approach to a Carnot cycle. Compression was made as purely adiabatic as possible, instead of as nearly isothermal as possible, as had usually been done in the embodiment of previous cycles. High efficiency being the main object in view, a wide range of temperature was needed, and this called for a similar range in pressure. The working pressure was therefore fixed as 500 pounds per square inch. As adiabatic compression to

1 "A Rational Heat-motor," by Rudolph Diesel. 
this pressure resulted in a temperature above that sufficient for the ignition of the fuel finally chosen, which was oil, igniting devices were omitted. The oil was simply injected into the cylinder when compression was complete, into an atmosphere above the temperature of ignition and in which, therefore, it could not help burning. The first result was a still further increase in temperature; but as a Carnot cycle demands isothermal addition of heat, Mr. Diesel invented his famous isothermal combustion, in which merely enough fuel was admitted to maintain a constant temperature as the gases expanded. This process replaced the ordinary temperature-raising combustion soon after the stroke commenced, and continued until the point in the stroke where the governor dictated a cut-off of fuel-supply. After this adiabatic expansion set in.

The general arrangement of the engine was similar to that of a vertical Otto-type engine, except that the cylinder was longer and narrower, and there was added an air-pump for creating a pressure on the surface of the oil sufficient to drive it into the compressed air within the cylinder when the valve opened. One impulse was received every two complete revolutions, as in the Otto engine.

The Diesel cycle is portrayed in Figs. 24 and 25 by the area $B C F G E^{\prime} B$, though a much higher pressure-limit than that drawn must be imagined in comparing it with the other cycles. The compression to 500 pounds is shown by $B C$, the primary combustion by $C F$, the isothermal combustion by $F G$, the adiabatic expansion by $G E^{\prime}$, and the exhaust at lower dead-centre by $E^{\prime} B$. It is obvious that the cycle is much nearer to a Joule than to a rectangular Carnot. It is further obvious that Mr. Diesel, in remembering the statement that the Carnot cycle was the one of maximum efficiency, forgot under what limitation that statement was made: "Under any given temperature-limits." For if 
some other cycle depart from the Carnot rectangle but at the same time attain a greater temperature-range, it may have an efficiency greater than that to which the original Carnot cycle was restricted. Thus, here, the isothermal $F G$, which would impart greater efficiency than the isomorphic $C D$, if the upper temperature-limit were $F G$, plainly decreases the efficiency when compared with that attained by admitting the heat beneath $F G$ along the isomorphic $F D$, to some point near $D$, before expansion sets in.

From these considerations it may be concluded that the maximum efficiency available under equal pressure-range is always greater in the Joule than in the Diesel cycle. Under equal temperature-limits either may be the greater; but to attain equal temperature-limits the Diesel cycle demands an egregiously superior maximum pressure. 


\section{CHAPTER VII}

\section{THE HOT-AIR ENGINES}

THE history of the hot-air engines, if the efforts of Hiero of Alexandria, who worked in the second century before the Christian era, and of a few unsuccessful inventors be excepted, is comprised between the years 1827 and 1854 . On the former date Robert Stirling took out his patent for a regenerative air-engine ; on the latter date Ericsson's hotair propelled ship, with its enormous engines, sank in New York harbor, never to rise again into a world of "caloric" engines. During this period the energy of many of the best minds of the mechanical world were devoted to its perfection. It passed from the status of a toy to a prime mover of hundreds of horse-power, and back again. It experienced all the glamour and romance to be had from youthful promise of the most fascinating sort. It grew to manhood under the guidance of master minds. It was tried and found wanting. It is now relegated almost solely to the task of supplying occasional country-residences and small hotels with water. The question of its worth for any larger task than this has been effectually sealed by one of the most valuable failures on the part of one of the ablest engineers that the world has ever seen, - that of Ericsson, who, after twenty years of effort, failed to produce a machine which could compete with the steam-engine in the world's field.

From the very start the hot-air engine offered an irresistible fascination for the inventor, because even the crudest attempts with it always resulted in an efficiency far superior 
to that of the steam-engine. This is readily explained as being due to the far greater temperature-range possible when working with a perfect gas than with steam, where the attempt to get high temperatures is always met by a need of impossibly high pressures. This contrast was of course more marked in those days, when 30 to 50 pounds per square inch was regarded as the highest steam-pressure practicable in stationary engines. Its final failure was due to phenomena which manifested themselves from the start and which are now known to be ineradicable. These phenomena are :

(I) The rapid deterioration of metallic surfaces when used to transmit high-temperature heat from an atmosphere of carbonic acid to a hot dry gas, such as air ;

(2) The large amount of such surface required to transmit a given amount of heat when compared with like transmission to water or to saturated steam; and

(3) The great bulk of the weight of air requisite for handling a proper amount of energy.

The first two obstacles have been obviated in the natural offspring of the hot-air engine, the internal-combustion gasand oil-engines. Incidentally the last has also been partly eliminated in the same way; though it is imaginable that the application to the hot-air engine of as great a workingpressure as is now common in the gas-engine might have been very beneficial to it in respect to bulk.

The Stirling Air-engine. - The utter impossibility of a practicable Carnot-cycle air-engine was pointed out at the end of Chapter V. The attainment of any practicable efficiency by means of any other cycle, with air as a workingsubstance, became only possible with the invention of the regenerator, by Stirling, in 1827 as a substitute for adiabatic compression and expansion for the transfer of the air from one temperature-level to another. The regenerator consists 
of a chamber containing a mass of heat-absorbing material arranged so as to present as large a surface as possible to a current of air passed through it. In practice this material was usually iron wire, arranged in a close network. Through this network the air was passed back and forth, giving up to the wire its heat as it entered from the hotter end and picking it up again as it passed back again from the colder refrigerative chamber of the engine. The only forms in which the regenerator survives in modern industry are the hot-blast "stoves" of the blast-furnace, and the air-heating surfaces of the regenerative gas- and oil-stoves and lamps. With all of the work in this line the name of Siemens is chiefly and actively connected.

The arrangement of Stirling's regenerative engine is shown in Fig. 26, in which the regenerator occupies the annular space $R R$ about the larger cylinder. When in operation the temperature of this generator at the lower end is $T_{1}$ and at the upper end $T_{2}$, being graded continuously from one to the other. Constructively, the engine consists of a crank-shaft and fly-wheel to which are coupled by connecting-rods the large "displacer piston" $D$ and the "working-piston" $W$, but in such a way that their strokes take place alternately. (Theoretically, the stroke of each should be complete before that of the other begins. In practice, each stroke laps over the other somewhat; but in analyzing the engine's action the first will be assumed to be the case.) At the lower end of the cylinder $D$ is placed the fire, in the furnace $F$, usually maintaining the iron at a temperature approaching a visible red, and at the upper end is the coil of condenser-pipes $C C$, filled with a current of cold water. The action is traced mathematically in the $N T$ and $P V$ diagrams of Figs. 27 and 28.

It were best noted at the start that the volume of air within the engine depends solely upon the position of the 
piston $W$. The motion of the piston $D$, which fits loosely in its cylinder, meets with no possible resistance and causes no change of volume, because the two ends of its cylinder are always open to one another.

The engine is shown in a position represented as midway between the points $A$ and $B$ in the diagrams, containing a

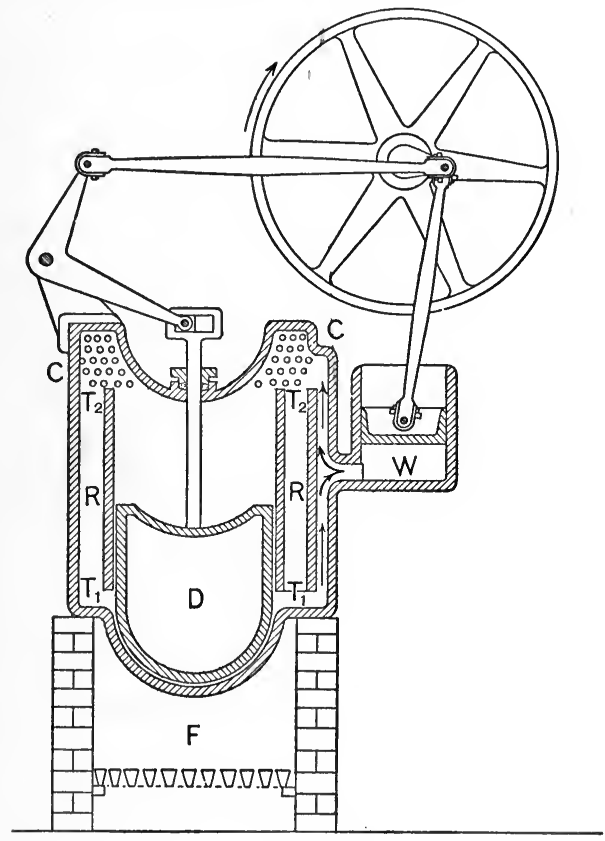

FIG. 26

quantity of air at approximately atmospheric pressure and minimum temperature. The air had been at atmospheric pressure at the beginning of $W$ 's downward stroke, at the point $A$. It has now been somewhat compressed, by the energy of the fly-wheel, isothermally because in contact with the cold pipes $C C$. This process is shown by the line $A B$, in both diagrams. At the end of $W$ 's stroke the 
volume is a minimum, and $D$ now moves upward, displacing the cold air from the space $C C$ through the regenerator $R R$ into the lower end of the cylinder. (The by-passing of the air around the regenerator through the ports leading to $W$ is properly prevented, sometimes by valves, sometimes by the small size of $W$ as compared with $R$ and $D$, and sometimes by making use of only one port.) This raises the

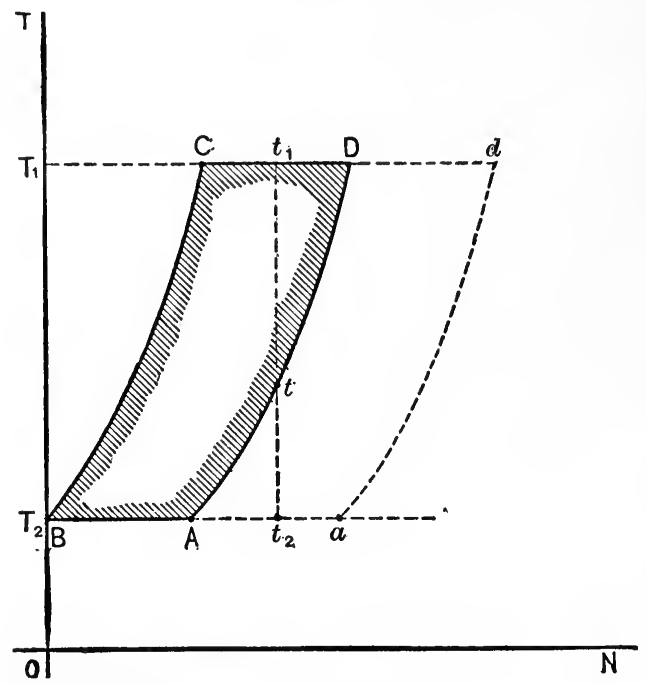

FIG. 27

air, at constant volume, from the pressure at $B$ to that at $C$ by reason of the change of temperature from $T_{2}$ to $T_{1}$. This is shown by $B C$ in both diagrams.

While $D$ is now quite at its upper dead-centre, $W$ moves up under the influence of the increased pressure and makes its working-stroke, the air expanding isothermally instead of falling in temperature adiabatically, because in contact with the fire-surface at the temperature $T^{\prime}$. This is shown by the line $C D$ in both diagrams. 
Then, while $W$ is quiet at its dead-centre, $D$ comes down, displacing the air again to the top of the cylinder through the regenerator, cooling the air under constant volume from $T_{1}$ to $T_{2}$ and reducing the pressure from $P_{D}$ to $P_{A}$. This is shown by the line $D A$ in both diagrams. The cycle is then complete.

Thus the displacer-piston $D$, with the aid of the regenerator, changes the pressure; the working-piston $W$, with

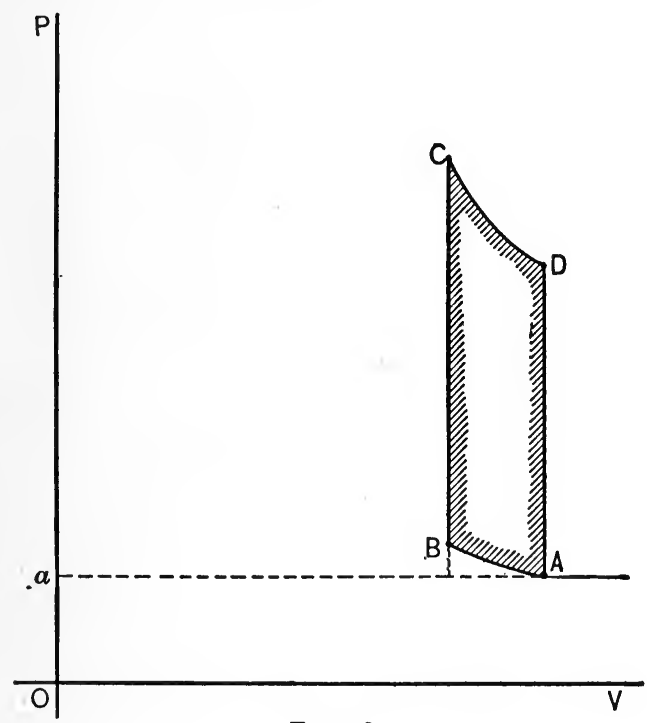

FIG. 28

the aid of fire and condenser, changes the volume, isothermally. This cycle may be summarized as follows :

I. $D$ at bottom ; $W$ moving down ; line $A B$ in diagrams ; negative work done (area under $A B$, Fig. 27).

II. $W$ at bottom ; $D$ moving up; line $B C$ in diagrams. III. $D$ at top; $W$ moving up (working-stroke); line $C D$. IV. $W$ at top ; $D$ moving down ; line $D A$ in diagrams. 
Problem 30. What are the conditions of operation and the efficiency of a Stirling engine operating with one cubic foot of atmospheric air, the temperature of the fire-surface being $1539^{\circ} \mathrm{F}$. and the volume of the rest of the engine being four times that of the cylinder $W$ ?

This gives the volume at $A=\mathrm{I} .00$ cubic foot, that at $B=0.80$ cubic foot. The temperature at $A$ and $B$ is $520^{\circ} \mathrm{F}$. absolute, and that at $C$ and $D$ is $2000^{\circ}$ absolute. The pressure at $A$ is 14.5 pounds per square inch.

$A B$ being an isothermal, $P_{B}=\frac{1.00}{0.80} \times 14.50=18.12$ pounds per square inch. In the heating at constant volume the pressures must change proportionally with the temperatures; therefore $P_{C}=\frac{2000}{520} \times 18.12=69.25$ pounds. $C D$ being an isothermal again, $P_{D}=\frac{0.80}{\mathrm{I} .00} \times 69.25=55.40$ pounds. The mass involved being 0.0758 pound and the heating being at constant volume, $N_{C}=0.00$ I30I. The heat abstracted along $A B$ must be equal to the (negative) external work done, or

$$
\begin{aligned}
Q_{2} & =\frac{\mathrm{I}}{778} \cdot P_{A} V_{A} \log _{e} r=\frac{C_{A} T_{A}}{778} \cdot \log _{e} \frac{V_{A}}{V_{B}} \\
& =\frac{53.18 \times 0.0758 \times 520}{778} \times \log _{e} \frac{\mathrm{I} .00}{0.80}=0.60 \mathrm{I} \mathrm{I} \mathrm{B.t.u.}
\end{aligned}
$$

The entropy absorbed along $A B$ must be this divided by 520 , or 0.00I156. Substituting $T_{D}$ for $T_{A}$ in the equation for $Q_{2}$ gives

$$
\begin{aligned}
Q_{1} & =2.3 \mathrm{I} 2 \text { B.t.u., } \\
Q_{w} & =Q_{1}-Q_{2}=\text { I.7 I I B.t.u., } \\
F & =\frac{Q_{v}}{Q_{1}}=74.0 \% .
\end{aligned}
$$

As to efficiency, it might seem from the diagram that the heat supplied by the fire should be the area beneath $B C D$, 
Fig. 27, and that that absorbed by the refrigerator should be the area beneath $D A B$. But of these quantities the heat beneath $B C$ enters the air, not from the fire, but from the regenerator, where it was stored up by the $D A$-process of the preceding cycle. Thus, while it might seem from the form of the cycle that the heat fell into the $B C$-line from the temperature $T_{1}$, and out of the $D A$-line into $T_{2}$, this is not the case, as will be evident if two succeeding cycles, such as $A B C D$ and $a A D d$, are considered. Each atom of entropy, such as that at $t$, which reaches the $A D$ line of the second cycle has done so by first falling from $t_{1}$ to $t$, doing work in the first cycle; it is deposited, without free fall, in the regenerator at this level. It is afterward picked up by the $A D$-process of the second cycle, in which it falls from $t$ to $t_{2}$, again doing work. So the heat supplied, $Q_{1}$, is only that beneath the line $C D$, and $Q_{2}$ is only that beneath $A B$. As the entropies of these two processes are equal, $\frac{Q_{1}}{Q_{2}}=\frac{T_{1}}{T_{2}}$, and the efficiency is that of a Carnot cycle, or, in this case, $74.0 \%$.

Engines operating upon this cycle were built as large as 50 to 75 horse-power before their bulk and the lack of durability of the heating-surfaces threw them out of competition with the steam-engine.

The Ericsson Hot-air Engine. - The Ericsson engine differed from the Stirling constructively in that, instead of the working-piston also performing the compression, while the displacer-piston meets with no resistance, both pistons are under pressure, the smaller one doing the compression and the larger performing the work. The displacement through the regenerator is performed by the pistons passing the compressed charge from the smaller to the larger. Thermodynamically the Ericsson differs from the Stirling in that the regenerative processes joining the two isotherms 
are under constant pressure instead of under constant volume. The cycle is that portrayed in Figs. 29 and 30. The intake of fresh cold air into the smaller compressing cylinder is shown at $a A$, Fig. 30, and does not appear in Fig. 29. It is then compressed isothermally to $B$ in contact with the refrigerating surface. It is discharged along $B b$, Fig. 30, into the regenerator, through which it passes on its way to the larger working-cylinder. There it ap-

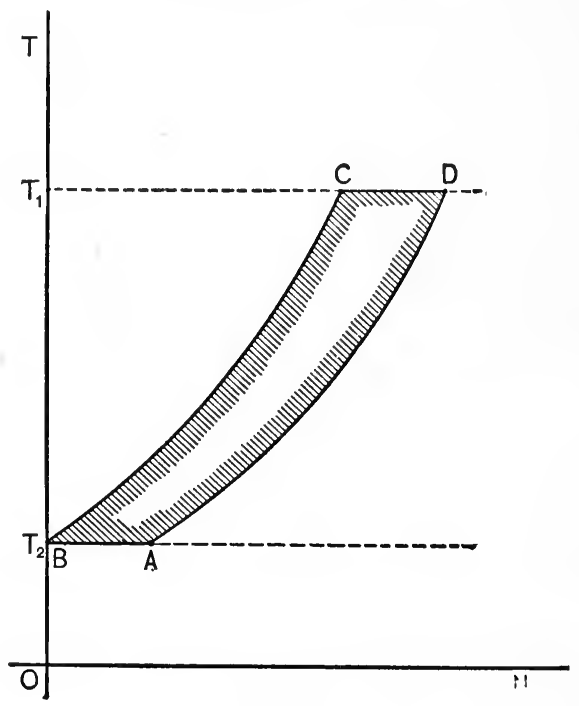

FIG. 29

pears as the volume $b C$, Fig. 30 , having in the interim been heated along the constant-pressure isomorph $B C$ of Fig. 29. At $C$ the working-cylinder experiences a cut-off of supply exactly as in the steam-engine, after which isothermal expansion takes place in contact with the fire-surface until atmospheric pressure is reached.

Constructively and thermodynamically the Ericsson bears somewhat the same relation to the Stirling that the Joule 
does to the Otto, except that the efficiencies of the two hot-air engines are the same, whereas those of the two gas-engines are not. Indeed, the diagram of the Joule engine, Fig. 23, will serve very well as an illustration of the general arrangement of the Ericsson engine if the chambers $T_{1}$ and $T_{2}$ be imagined as regenerators, each having its end toward $C$ at the temperature $T_{2}$ and the end

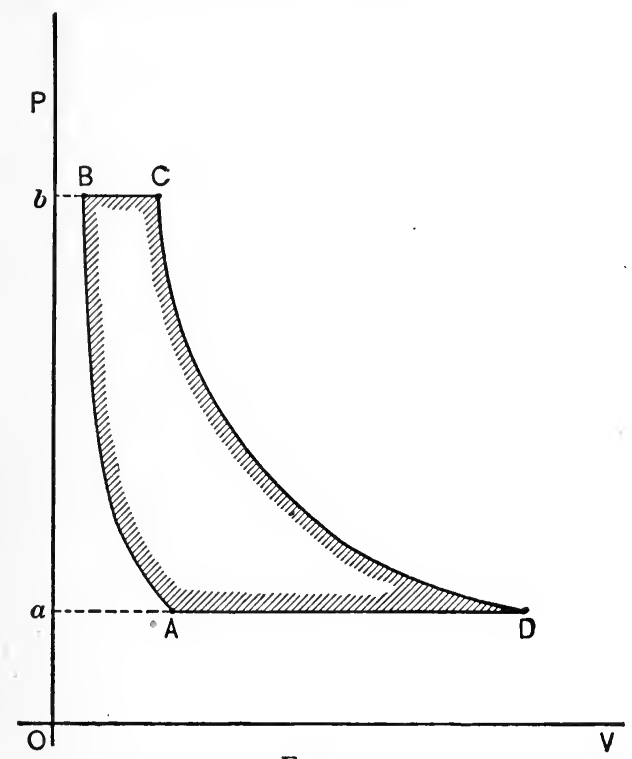

FIG. 30

toward $W$ at the temperature $T_{1}$, and if the cylinder $C$ be imagined as jacketed with cold water and the cylinder $W$ as surrounded with fire; for the working-cylinders of the Ericsson engines were actually hung over furnaces, much in similitude to huge camp-kettles. Another difference between the two hot-air engines was that in the Stirling the motion of the two pistons had to be properly timed, one with the other, to produce the proper effect, whereas 
in the Ericsson they were, within certain limitations, independent.

Problem 3I. What are the conditions of operation and the efficiency of an Ericsson engine working under the same temperature and pressure ranges as the Stirling engine of Problem 30 ?

Here $V_{A}=\mathrm{I}$.000. Since $P_{B}$ is to be 69.25, $V_{B}=0.2097$. Since $T_{C}=2000$ and the heating is under constant pressure, $V_{C}=\frac{2000}{520} \times 0.2097=0.8073$, and $N_{C}=0.02417$. The heat supplied along $C D=C_{C} T_{C} \log _{e} \frac{P_{C}}{P_{D}} \cdot \frac{\mathrm{I}}{778}$, from which $Q_{1}=$ I6. I8 B.t.u. and $N_{C D}=0.00808 . N_{D}=0.03225 . \frac{V_{C}}{V_{B}}=\frac{T_{C}}{T_{B}}$, whence $V_{C}=0.8073 . \frac{V_{D}}{V_{C}}=\frac{P_{C}}{P_{D}}$, whence $V_{D}=3.847 .{ }^{\circ}$ From the same argument as in Problem 30, the efficiency must be given by $\frac{T_{1}-T_{2}}{T_{1}}=74.0 \%$. The amount of heat converted into work, or $Q_{w},=0.74 \times$ I6.1 $8=$ I I.97 B.t.u.

The engines of the vessel Ericsson, the culmination of that engineer's efforts, well illustrate the reasons for the failure of the hot-air engine. Though designed to propel a vessel of 260 feet in length at the modest speed of seven miles per hour, the bulk and weight of the engine was enormous. It had four compressing-cylinders, each $11 \mathrm{I} .4$ feet in diameter, and four working-cylinders, each 14 feet in diameter; the stroke of each was 6 feet. The vessel sank before accurate tests of efficiency or capacity could be made, but as the maximum working temperature is mentioned as being about $443^{\circ}$, the theoretical efficiency could not be above $42.6 \%$, and the actual efficiency must have been less than half of this, or not over $20 \%$. As this is very much better than the best marine engines even of to-day can do, there is little wonder that the new "caloric" engine won tremendous applause as the superior 
of the steam-engine. But the difficulty of floating engines powerful enough to give the hull a practicable speed, even with the weight saved in coal-bunkers and boilers and the constant trouble and rapid deterioration due to attempting to lubricate the pistons under such (then) unusually high temperatures, were so obvious that the Ericsson, though promptly raised to the surface, was reëngined as a steamer, and the attempt has never been repeated. 


\section{CHAPTER VIII}

\section{SUMMARY OF THEORETIC POSSIBILITIES OF VARIOUS TYPES}

THE efficiency of a heat-engine is only one, and often a minor one, of the many factors which determine its value. The one always of great, and often of the first, importance is the capacity for developing power within a given volume, weight, or cost of machinery. Usually the qualities coming next in the order of importance are simplicity, reliability, and adaptability to varied service. But, confining the attention entirely to mere thermodynamic considerations, it is interesting to note the maximum possible efficiency of the several types when compared with what may be called the "builder's factor," viz.: the volume of cylinder (in cubic feet) times the maximum pressure upon the piston (in pounds per square inch), divided by the work developed (measured in heat-units), which is as accurate an indication of the cost per horse-power as can be based upon purely thermodynamic conditions.

The higher the builder's ratio, the greater will be the cost of the engine per horse-power.

This table shows clearly, if coupled with the statements of the obstacles arising in attempting to utilize hot air, how those engines of the highest efficiency pay for it in bulk and cost, and, vice versa, how those of high capacity (or low cost) pay for it in poor efficiency. It will be noticed that all of the engines using permanent gases for working- 


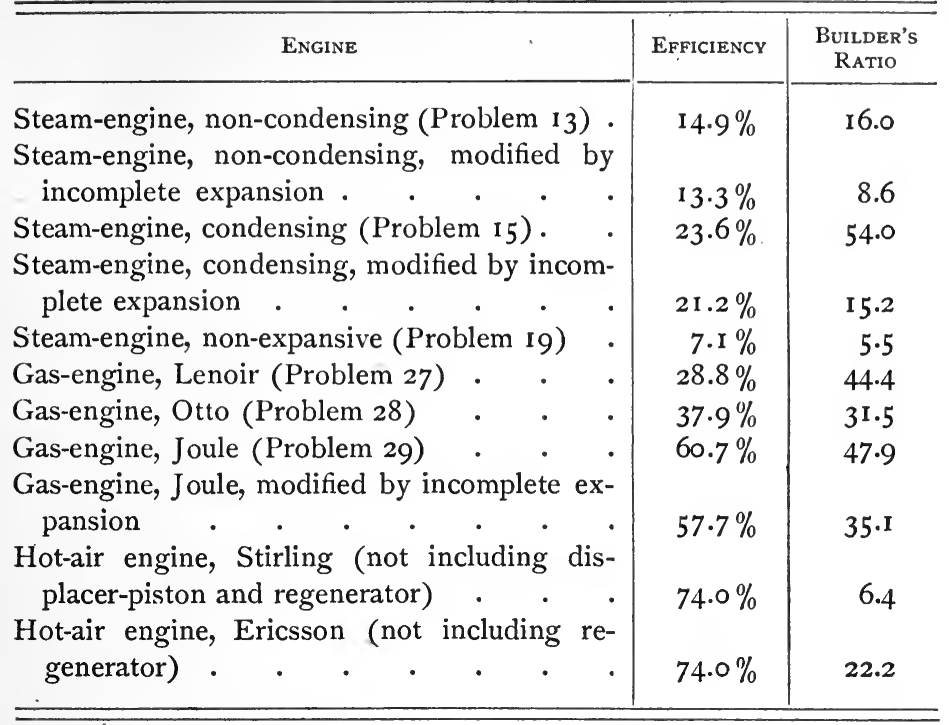

substance exceed the non-condensing steam-engine in cost as well as in efficiency.

It should be noted that all of the engines which expand to atmospheric pressure end the indicator-diagram with a sharp toe; this calls for an exaggerated maximum volume without sufficient pressure, toward the end, to develop commensurate power. This trouble is always obviated in practice by ending the stroke with an appreciable pressure still in the cylinder, which allows the engine to make a better showing in regard to builder's ratio ; this stands quite aside from those considerations which affect all of the engines in passing from theory to practice. Thus, by ending the expansion in Problem I 3 at I 5 pounds per square inch of net pressure the efficiency is reduced only from $\mathrm{I} 4.9$ to $\mathrm{I} 3.35 \%$, while the builder's ratio falls from I6.0 to 8.6. Similarly, ending the expansion in Problem 15 at 7 pounds per square 
inch net pressure reduces the efficiency only from 23.6 to $21.2 \%$, while the builder's ratio falls from 54.0 to $15.2 \%$. Shortening the maximum volume of the Joule cycle to 2.5 feet decreases the efficiency only from 60.7 to $57.7 \%$, while the builder's ratio falls from 47.9 to quite near that of the Otto. 


\section{CHAPTER IX}

\section{THE REFRIGERATING MACHINES 1}

As in the case of the clockwise cycle, the reversed thermodynamic or refrigerating cycle does not exist in practice in the rectangular or perfect form. Practical considerations make it more profitable to accept a lessened thermodynamic efficiency for the sake of a saving in complexity, cost, bulk, etc.

The processes in actual use for the development of artificial cold may be divided broadly into two classes :-

I. Those depending upon the capacity of water for absorbing certain vapors at lower temperatures, which vapors may be driven off again by the addition of heat. They are called absorption-machines. "They demand no supply of mechanical power, the heat-energy which keeps them in operation being supplied in the form of heat directly to the water, and they involve no more moving machinery than one or two circulating-pumps. They therefore exhibit no purely thermodynamic phenomena, and will be given no further consideration in these pages.

II. Those operating on some thermodynamic cycle such as has just been discussed, and always involving a supply of power from without to keep them in operation. They are known as compression-machines. They may be subdivided into two sub-classes :-

(a) Those using a permanent gas, usually air, as a working-substance ; and

1 For the general laws applying to the reversed cycle the student is referred to pages 55 to 62 . 
(b) Those using a saturated vapor, usually that of anhydrous ammonia, ether, carbonic acid, etc.

Of these two sub-classes the air-machine is restricted almost wholly to use on shipboard. Of the vapor-machines, probably $95 \%$ rely on anhydrous ammonia as their workingsubstance.

The air-machines used purely for refrigeration always work on a reversed Joule cycle. Referring to Figs. 23, 24 , and 25, suppose that the shaft of the engine receives power from without which suffices to rotate it in a reversed direction. The direction of flow of the air, as indicated by the arrows, will also be reversed. In Fig. 25 let $A E$ represent a volume of atmospheric air taken in by the larger cylinder at a temperature of $520^{\circ}$ absolute. Its thermodynamic condition is shown at $E$, Fig. 24. Let it be compressed adiabatically to $D$. Its temperature will be $1342^{\circ}$ absolute, or $88 \mathrm{I}^{\circ} \mathrm{F}$. Let the air now be passed through the chamber $T_{1}$, which has now become a "refrigerator" by being equipped with coils of pipe containing a circulation of cold water, until its temperature has been reduced to $59^{\circ} \mathrm{F}$. again, or $520^{\circ}$ absolute. Its volume at $E$ being unity, that at $D$ is 0.1039 and that at $C$ is 0.059 I. Let this compressed air under ordinary temperature now enter the smaller cylinder and reëxpand there to atmospheric pressure. Its volume will now be 0.584 cubic foot and its absolute temperature $204^{\circ}$, or $257^{\circ}$ below zero Fahrenheit. It may now be exhausted into the room which is to be cooled at atmospheric temperature.

Of course, in actual practice no such low temperature as the one just calculated is attained. The pressure-limits which were chosen as suitable for the heat-engine of Problem 29 are far too great for the refrigerating-machines; the cold produced is far greater than that desired for the preservation of meat, etc., and the machine itself would 
freeze up in attempting to produce it. But the figures will illustrate how a Joule engine can be reversed for the production of cold.

It is obvious from Fig. 25 that power must be supplied to keep the cycle in operation. The positive work developed, the area $c C B A$, is much smaller than the negative work absorbed in compression, the area $A E D c$. As to efficiency, that depends upon the temperature of the refrigerated room itself, which is represented in Fig. 24 by the $x X$-level. The heat taken from the room is that beneath the curve $B X$. That beneath $X E$ represents leakage of heat from atmospheric temperature into the workingsubstance. Again, the heat is discharged, along $D C$, into water which cannot have a temperature higher than the $C$-level; therefore all of the area above that level represents wasted work, - similar to that of the man who supposedly lifts a barrel on his shoulder in order to raise it to a platform situated a foot above the ground. As $B E D C$ represents the work done, the efficiency must be the area $O B X z$ divided by the area $B E D C$, obviously a much poorer efficiency than the maximum possible, or that of a rectangle lying between the temperature-levels of $C$ and $X$.

The Ammonia Compression Machine. - The class of refrigerating machines to which the ammonia compression machine belongs comprises the great majority of those used in industrial enterprise. All of the largest machines are of this type. Its action also typifies that of all other machines making use of a liquid refrigerant which is evaporated into a saturated vapor and then returned to liquid form by compression and condensation.

The operation of the machine is illustrated diagrammatically in Fig. 3I. At the left is seen a "liquid-reservoir," which holds the stock of refrigerant (in this case liquid anhydrous ammonia) under a pressure sufficiently heavy to 
eliminate all danger of vaporization under all ordinary temperatures. The liquid exists, in fact, at atmospheric temperature, and may be carried in this condition in unlagged pipes to any locality, however distant, without any loss of its refrigerative capacity. Leakage, of even the most minute character, must always be guarded against, on account of the penetrating and unpleasant character of the pungent odor of ammonia, and also on account of the

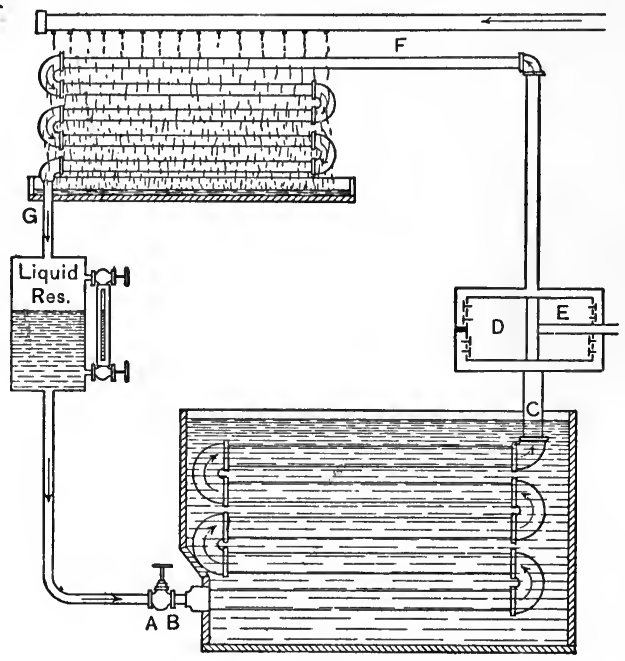

FIG. $3^{\text {I }}$

expense involved in maintaining the stock of ammonia in the face of leakage. The liquid-reservoir is shown equipped with a gauge-glass whereby the condition of the stock of liquid may be seen at a glance.

From the liquid-reservoir the ammonia passes, through a pipe known as the "liquid-line," to one or any number of expansion-valves, situated wherever refrigeration is desired. One of these expansion-valves is shown at $A B$. Upon escaping through this valve, which is usually a needle-valve 
of quite fine construction, the liquid finds itself freed from the so-called "head-pressure" of something like 160 to 200 pounds per square inch which kept it in its liquid condition. Beyond this valve it is under the comparatively light "backpressure" of about I 5 pounds by gage which prevails in the coil $B C$. The boiling-point of ammonia under this pressure is about zero Fahrenheit. In consequence, so long as the ammonia finds itself in contact with anything warmer than this temperature it will extract heat from it which is absorbed in the form of latent heat of vaporization. In exactly similar fashion a tea-kettle filled with water, finding itself in contact with a surface hotter than $212^{\circ} \mathrm{F}$., will abstract heat from it with which to vaporize the water; nor can the metal of the tea-kettle be brought to a higher temperature than $212^{\circ}$, by the application of ever so much heat, so long as there still be water in it.

In this way the ammonia cools the pipes $B C$ so nearly as it can to zero Fahrenheit, and with them anything in contact with their outer surface. The coil $B C$ may be situated in the room to be refrigerated, but more commonly it is placed in a tank of strong brine, as shown in Fig. $3 \mathrm{I}$, which is chilled to a temperature of $10^{\circ}$ to $20^{\circ} \mathrm{F}$., and is then circulated through pipes in the rooms to be refrigerated, or around cans containing pure water to be frozen into ice. The object in interposing the brine between the real refrigerating process and the object to be refrigerated is to obtain stability of temperature. The slightest touch of the valve $A B$ will produce considerable variation in the refrigerative activity of the coil $B C$. With the great mass of brine present all such variations are absorbed without producing a correspondingly fluctuating effect upon the object to be refrigerated.

So long as any liquid ammonia remains unevaporated in the coil $B C$ it maintains its temperature at zero Fahrenheit. 
Immediately upon complete evaporation, however, the higher temperature of the brine produces superheat, and this is increased by the heat of the atmosphere as the ammonia-gas passes from $C$ to the compressor at $D$, a transit which may cover many feet of pipe.

In the compressor $D E$ the pressure of the ammonia-gas is raised from the back-pressure to the head-pressure again,

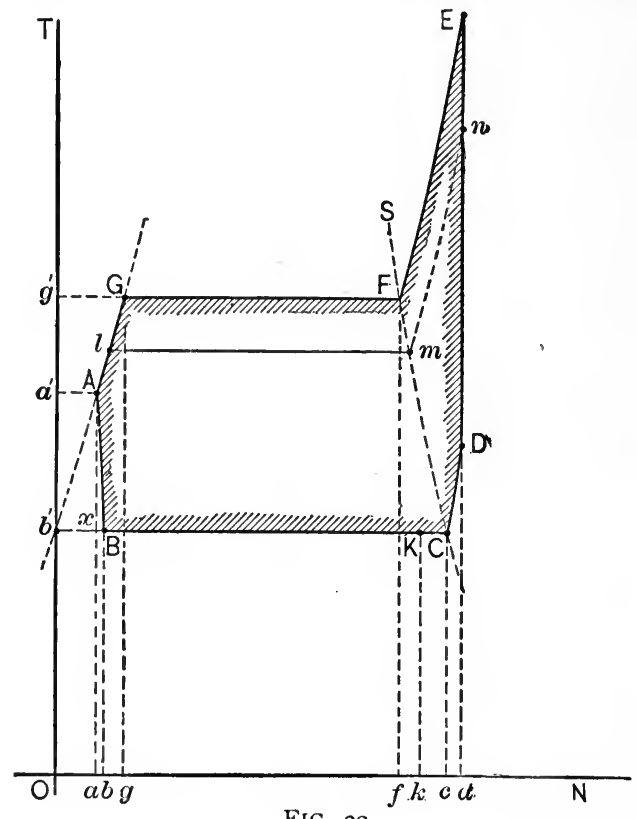

FIG. $3^{2}$

and under this heavy pressure the hot, dry gas is passed through the condenser-coils $F G$, over which is running a circulation of cold water. The chill of the water first reduces the temperature of the gas to the boiling-point under the head-pressure, and then, since this is always hotter than the water, condenses it back into its liquid form. From the condenser $F G$ the liquid trickles down into the 
liquid-reservoir, or receiver, as it is sometimes called, where it awaits use over again.

It is to be noted that, going in the direction of the flow of the ammonia, the back-pressure prevails from the expansion-valve $A B$ to the compressor $D E$, while the headpressure prevails from the compressor $D E$ to the expansionvalve again. This divides the system between two distinct pressures. The first, or lower, is so regulated as to maintain the proper temperature in the coil $B C$; this is done by adjustment of the expansion-valve and sometimes by vary-

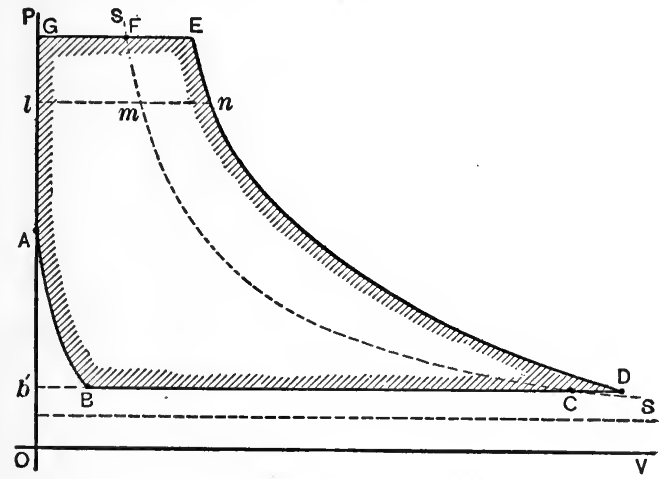

FIG. 33

ing the speed of the compressor. The second, or higher, pressure depends upon the temperature and amount of cooling-water supplied; it regulates itself more or less automatically, rising, under the influence of the discharge from the compressor, until the increased temperature makes the transfer of heat to the water sufficiently rapid to maintain thermal equilibrium.

The thermodynamics of the process is shown in Figs. 32 and 33. The point $A$ represents the primary condition of the liquid as it approaches the expansion-valve $A B$. The passage through the valve, which consists of simple wire- 
drawing or throttling, is shown by the constant-heat curve $A B$. It is evident from this that a portion of the refrigerative power of the ammonia is consumed in reducing its own temperature to its boiling-point, before any can be available for refrigerating other bodies. At the point $B$, when the ammonia is first ready to chill the coil $B C$, the proportion $\frac{b^{\prime} B}{b^{\prime} C}$ has already been vaporized and is incapable of further refrigerative activity.

As the ammonia traverses the coil $B C$ it is gradually evaporated under the constant back-pressure, as shown by the line $B \dot{C}$. When vaporization is complete at $C$, which is usually accomplished before the ammonia leaves the coil, superheat begins. It ends as the gas reaches the compressor in the condition $D$.

The next step consists of the adiabatic compression of the gas in the compressor, as shown by the line $D E$, the gas being discharged from the compressor in a condition of high superheat.

As the gas flows from the compressor through the condenser-coils its temperature is first reduced from $E$ along the line $E F$, it is then condensed isothermally to liquid form along the line $F G$, and finally it is cooled as a liquid, during its stay in the receiver and on its way to the expansion-valves, along the curve $G A$. The cycle is now complete.

The efficiency, capacity, etc., may be calculated as in the other cycles. It is to be especially noted, however, that whereas in the heat-engine cycles the thing supplied is heat, or $Q_{1}$, and the thing wanted is work, or $Q_{w}$, and hence the efficiency is $\frac{Q_{v}}{Q_{1}}$, now the thing supplied is work, or $Q_{w}$, and the thing wanted is refrigeration, or heat-abstracted, or $Q_{2}$, and hence the efficiency of a refrigerating- 
machine is given by the expression $\frac{Q_{2}}{Q_{w}}$. But since the former is not a portion of the latter, the two being quite independent quantities of energy, the ratio just expressed is not properly an efficiency at all, but a sort of coefficient of efficiency. It is none the less useful, and custom has warranted its continuance.

To illustrate, let a set of conditions be assumed.

Problem 32. What will be the amount of refrigerative effect and the efficiency of operation if one pound of liquid anhydrous ammonia at a temperature of $69^{\circ} \mathrm{F}$. and a pressure of 2 I0.7 pounds absolute be evaporated under a back-pressure of 29.74 pounds absolute, the temperature of the gas reaching the compressor being $39^{\circ} \mathrm{F}$.?

Reference to Figs. 32 and 33 and to the ammonia-tables ${ }^{1}$ places at hand all of the necessary guides and data for calculating all of the heat-quantities desired. The values of abscissæ and ordinates for the various points of the cycle are tabulated below. The manner of their calculation is as follows

\begin{tabular}{|c|c|c|c|c|c|}
\hline Point & $\begin{array}{c}\text { Pressure } \\
\text { Lbs. p. sq. in. abs. }\end{array}$ & $\begin{array}{l}\text { Volume } \\
\text { Cubic feet }\end{array}$ & \multicolumn{2}{|c|}{$\begin{array}{l}\text { TEMPERATURE } \\
\text { Fahr. F. Abs. }\end{array}$} & ENTROPY \\
\hline$A$ & I04.9I & 0.109 & 59 & 520 & o. I 325 \\
\hline$B$ & $29 \cdot 74$ & 0.835 & 0 & $46 r$ & 0.1401 \\
\hline$C$ & 29.74 & 9.63 & 0 & $46 I$ & I.2277 \\
\hline$D$ & 29.74 & I0.44 & 39 & 500 & I. 2689 \\
\hline$E$ & 210.7 & $2.36 \mathrm{I}$ & 340 & 801 & I.2689 \\
\hline$F$ & 210.7 & I.59 & 100 & $56 \mathrm{I}$ & I.0826 \\
\hline$G$ & 210.7 & 0.109 & 100 & $56 \mathrm{I}$ & $0.2 \mathrm{I} 6 \mathrm{I}$ \\
\hline$K$ & 29.74 & 8.877 & 0 & $46 I$ & I.I 317 \\
\hline
\end{tabular}

The cycle is most readily understood if assumed to start at $A$, where the pressure upon the liquid first becomes low ${ }^{1}$ See Peabody's “Steam-Tables," Table X, for instance. 
enough to permit vaporization. The path $A B$ is a constant-heat curve of wire-drawing, whence the heat at $B$, or the area $O b^{\prime} B b$, must equal that at $A$, or the area $O b^{\prime} A a$. From the ammonia-tables the latter is found to be 64.6 B.t.u., reckoning from an arbitrary zero (for this problem) of zero Fahrenheit, at $b^{\prime}$. Therefore, in Fig. 32,

$$
b^{\prime} B=64.6 \div 46 \mathrm{I}=0.140 \mathrm{I} \text {. }
$$

The path $B C$ represents the isothermal vaporization in the coils $B C$. The area $O b^{\prime} C c$ must represent the latent heat of vaporization, given as 566 B.t.u.; whence $b^{\prime} C=1.2277$. The path $C D$ represents the superheat on the way to the compressor and has the equation

$$
N-N_{C}=S \log _{e} \frac{T}{T_{C}}
$$

The path $D E$ shows the adiabatic compression in the compressor, and $E F G A$ the cooling under constant pressure in the condensing-coils and in the liquid-lines. For these latter curves one must know the specific heats of ammonia under constant pressure : $\mathrm{r} .095$ for the liquid and 0.522 for the vapor, and the ratio of the two specific heats for the vapor: I.3I or 1.317. Since all of the conditions of the vapor studied in the present problem lie close to the saturation-point, and consequently the specific heats are not constant, the laws governing these curves will not be found in exact agreement, in their mathematical results, with the ammonia-tables; but the variations will not be sufficient to affect the accuracy of the study from an engineering standpoint.

The determination of the heat-quantities handled, $Q_{1}, Q_{2}$, and $Q_{w}$, requires little discussion. $Q_{1}$, the area $a A G F E d$, is readily found to be the sum of the heat of the liquid $(a A G g=45.4)$, of the latent heat $g G F f(=486.0)$, and of the heat of superheat $f F E d(=157.0)$, or 688.4 B.t.u. $Q_{w}$, 
the area $A B C D E F G A$, can only be found as the difference between $Q_{1}$ and $Q_{2}$, the area $a A B C D d$. In $Q_{2}$ occurs the area $a A B b$; reference to page 108 gives it as 4.3 B.t.u. The area beneath $B C$ must be the latent heat of vaporization along $b^{\prime} C$ minus the heat of the liquid between $b^{\prime} A$; because the area $O b^{\prime} B b$ must equal the area $O b^{\prime} A a$. The heat of superheat beneath $C D$ is readily found from the specific heat and the range in temperature.

From these data $Q_{2}$ is found to be equal to the sum of 4.3, 501.4, and 20.3 B.t.u., or 526.o B.t.u. This makes $Q_{v}=162.4$ B.t.u. But of this thermodynamic value of $Q_{2}$ only the area $b B C c$ is useful refrigeration; therefore the efficiency-coefficient of the cycle is

$$
\frac{b B C c}{A B C D E F G}=\frac{501.4}{162.4}=3.087 \text {. }
$$

It has been pointed out by Mr. William Lee Church that the efficiency of the cycle might be improved if the waste cold lost in the transfer of gas from refrigerating-coil to compressor, $c C D d$, might be used to chill the liquid to its boiling-point before it is released through the expansionvalve, by jacketing the pipe leading to the valve $A B$ (Fig. 31) with the pipe $C D$. This would result in bringing the cycle down the path $G A b^{\prime}$ to the lower level instead of down the path $G A B$. $Q_{1}$ would now become 753 B.t.u., $Q_{2} 586.4$ B.t.u., and $Q_{w}$ I66.6 B.t.u. But the cold $c C D d$ is not sufficient to absorb all of the heat $O b^{\prime} A a$; in order to carry frost visibly through this jacket-modification some liquid must be allowed to boil over into it, sufficiently to add to the cold an amount, $k K C c$, large enough to make the area $k K C D d=O b^{\prime} A a$. From these considerations $K C$ is found to be 0.0960 , making $b^{\prime} K=1.1317$ and the area of useful refrigeration, $O b^{\prime} K k,=52$ I.7 B.t.u. The efficiencycoefficient is therefore now equal to $52 \mathrm{I} .7 \div \mathrm{I} 66.6=3 . \mathrm{I} 3 \mathrm{O}$, 
a result some $1.4 \%$ better theoretically than that deduced above. In practice the gain is greater than this.

The Series Refrigerating Process. - The ordinary industrial demand for artificial cold is for cold of a very moderate degree. The rooms of a cold-storage warehouse for the preservation of meats and other provisions which must not be frozen are kept as closely as possible at a temperature of $34^{\circ}-36^{\circ} \mathrm{F}$. Those where fish, butter, etc., are frozen need to be only slightly below $32^{\circ} \mathrm{F}$. Either of these duties are usually performed by a circulation of brine not colder than $20^{\circ} \mathrm{F}$. and the same medium at the same temperature serves to freeze artificial ice. The brine is maintained easily at this temperature by a refrigerant having no lower a temperature than $0^{\circ} \mathrm{F}$.

But for scientific purposes the need of obtaining much lower temperatures than these has long been urgently felt, though the amount of heat to be handled is naturally small. For none of these purposes is the Joule air-cycle fit. The refrigerant is too diffuse. All of the successful methods which were formerly relied upon for very low temperatures made use of the ebullition under reduced pressure of some liquid whose boiling-point was unusually low even under atmospheric pressure. The locality to be refrigerated, usually a small piece of laboratory-apparatus, could be surrounded by the liquid and the precious cold conserved to the last degree.

The liquids commonly used were carbonic acid or ether, operated upon the cycle of the ammonia-machine. But even under vacua these liquids would not absorb heat at a sufficiently low temperature. Recourse was then had to a thermodynamic series. Liquid carbonic acid, boiling under reduced pressure, would produce a degree of cold which, aided by high pressure, would condense some more refractory vapor, its heat being absorbed by the boiling 
carbonic acid. This refractory liquid, when once the pressure upon its surface were relieved, would boil and absorb heat at a much lower temperature than would the carbonic acid. This process is the thermodynamic obverse of the Series heat-engine.

By this means many of the so-called permanent gases were liquefied in scientific laboratories and much of inestimable value was accomplished. But the limitations of the process were found, in complication, expense, and rapidly diminishing efficiency, and rapidly increasing labor with which even the smallest quantity of heat could be abstracted at the lowest temperatures, at a point which still fell far short of attaining degrees of cold for which scientific investigators felt urgent need. The entire situation has now been solved by the Linde liquid-air process, which can of course be applied to the liquefaction of other "permanent" gases almost as readily as it can to that of atmospheric air.

Lord Kelvin's Warming-machine. - It has been pointed out by Lord Kelvin that whereas, in the ordinary refrigerating-machine, the absorption of the heat $Q_{2}$ is the thing desired, the development of the discharged heat $Q_{1}$ being an undesired incident in the process, this aspect of the cycle might be reversed by applying $Q_{1}$ to the heating of buildings: Indeed, the machine might be wholly devoted to this purpose, in which case $Q_{1}$ becomes the end in view and $Q_{2}$ becomes the incidental factor. Thus, instead of applying the heat developed from burning coal directly to the rooms to be heated, this heat might be used in a heatengine to develop power, the exhaust from the heat-engine being utilized for heating the building; the power so developed might then be utilized in driving a refrigeratingmachine which absorbed low-temperature heat from out-ofdoors and discharged its high-temperature heat into the 
building. The proposition might be paralleled, for elucidation, to that of a man wishing to fill a land-locked basin lying some few feet above the sea-level with water and having, as a means with which to work, a small stream falling from a mill-pond situated ten times as high as the basin above the sea. He might, in the first place, simply let the stream flow into the basin, which it would eventually fill. This would correspond to the ordinary method of heating buildings. But if his small water-power were extremely valuable, or if time were valuable also, he might hasten matters by letting the small stream, in its fall into the basin, drive a water-wheel. By attaching to this waterwheel a pump piped to lift water from the sea and to discharge it into the basin, it is plain that the latter would be filled much more quickly and at a much less cost of millpond water. If the basin were Io feet and the small millpond 100 feet above the sea-level, respectively, the basin would be filled in the second case in one-tenth of the time, or with one-tenth as much water drawn from the mill-pond, as compared with the first case. To illustrate the proposition thermodynamically the following problem will be useful :-

Problem 33. What will be the comparative amount of heating accomplished ( $\mathrm{I}$ ) by applying a given amount of heat directly to a building, and (2) by making use of a Kelvin warming-machine made up of the steam-engine of Problem I4 driving the ammonia compression machine of Problem 32? (In this, the temperature out-of-doors is supposed to be that of the $B C$-level of Fig. 32.)

According to Problem I4, for every IOO B.t.u. supplied to the engine 23.6 B.t.u. are transformed into work. The remaining 76.4 B.t.u. may be imagined as exhausted into the building at a temperature of $13 \mathrm{I}^{\circ} \mathrm{F}$. According to Problem 32 (2d Case) the supply of 23.6 B.t.u. of work 
will absorb from the lower level of zero Fahrenheit and discharge at the higher level of $100^{\circ} \mathrm{F}$.: $3.13 \times 23.6$ $=73.9$ B.t.u. of low-temperature out-door heat. This, added to the 23.6 B.t.u. of work which is converted into heat within the refrigerating-machine and to the 76.4 B.t.u. of exhaust-heat from the engine, makes a total of 173.9 B.t.u., which reach the building from the original supply of 100 B.t.u. The efficiency of the fuel has been increased by $73.9 \%$.

A little consideration will show that this effect does not stand as an infraction of the law of the conservation of energy, as may at first appear, but as a combination of the operation of that law within the heat-engine with the operation of the second law of thermodynamics in the refrigerating-machine. No heat has been created. Heat has been merely lifted up-temperature from out-of-doors into the building.

This plan may be best imagined as put into practice by heating the building with hot-water pipes filled with the circulation coming from both the steam and the ammonia condensers. It is, of course, impracticable at present by reason of the great cost of apparatus and of attendance. But it is by no means improbable that at some time during the present century, when coal shall have become much dearer and machinery much cheaper and more simple than now, the plan may be relied upon for the heating in winter (and the cooling in summer) of our larger buildings. 

PART II

APPLICATION OF THEORY TO PRACTICE 



\section{CHAPTER I \\ THE SIMPLE STEAM-ENGINE}

WiTh a full understanding of the theoretical laws which limit and control the development of power from heat in the various types of engines in use, it is next in order to discuss in what points the operation of these theoretically perfect engines are modified by the limitations of actual construction and by those thermal phenomena, such as the conductivity of the cylinder-walls, etc., which have been carefully excluded from the foregoing analyses.

The theoretical cycles are modified by these factors in two distinct ways, either of which may be of prime importance in any given case :-

(I) In Efficiency, or in the proportion of the heat theoretically available for work which is actually developed; and

(2) In Capacity, or in the amount of power available from a given size or weight of engine.

The first characteristic is customarily expressed, in regard to any given engine, by a ratio called its cylinderefficiency. Following the notation of the first portion of the book, this cylinder-efficiency may be defined as follows: Let the heat supplied to the engine be represented by $Q_{1}$, that abstracted by the refrigerator by $Q_{2}$, that theoretically available for conversion into work by $Q_{w}$, and that actually converted into work by $q_{w}$; then the expression for the cylinder-efficiency would be -

$$
F_{c}=\frac{q_{w}}{Q_{w}}=\frac{q_{w}}{Q_{1}-Q_{2}} .
$$


This expression is a valuable one for the indication of the proper place of the engine upon the efficiency-records. It covers the skill with which the designer and the builder together have been able to develop within the cylinder a proper portion of the work which the thermodynamic limitations permitted them to expect. It sweeps away all the complexity and ambiguity involved in attempts at deduction from the varied conditions of actual operation to a common basis of comparison for engines of any sort. Not only do the most diverse types of steam-engine fall into line for such equity of comparison, but gas-engines or hot-air engines lend themselves to the same process with equal docility and permit themselves to be placed thereby upon the same platform with the steam-engines without injustice to either.

For instance, it happens that a common value of cylinder-efficiency for simple single-cylinder steam-engines and for Otto gas-engines is $65 \%$. That is, of the work theoretically available in each case the designer is able to develop about two-thirds. This proportion sometimes rises above $70 \%$; it more frequently falls to $60 \%$, or even below. It is also an invariable rule that this proportion varies inversely with the engine's complexity. That is, it is highest in simple engines, lower in double compound, and lowest in triple compound engines.

The only other factor needed to express the value of the engine as a means of development of power is the efficiency with which the work developed within the cylinder is transmitted to some useful destination, usually the belt. The loss in transmission is, of course, due solely to enginefriction. The ratio of work arriving at the belt to that developed upon the piston is referred to as the mechanical efficiency of the engine. If we represent the work thus transmitted to the belt by $q_{b}$, the several efficiencies might be expressed as follows :- 


$$
\begin{aligned}
& \text { (I) Cycle-efficiency }=\frac{Q_{w}}{Q_{1}} ; \\
& \text { (2) Cylinder-efficiency }=\frac{q_{w}}{Q_{w}} ; \\
& \text { (3) Thermodynamic efficiency }=\frac{q_{b}}{Q_{1}} \text { or } \frac{q_{w}}{Q_{1}} ; \\
& \text { (4) Mechanical efficiency }=\frac{q_{b}}{q_{w}} \text {. }
\end{aligned}
$$

Only the last three are ordinarily needed or used. As to the thermodynamic efficiency, there is a lack of unanimity as to whether the belt-work or the indicated work should form the basis for comparison; but the only grounds for adhering to the latter are a custom grown up from an unfortunate mistake in the past. All of the arguments are in favor of referring to belt-power only. If this is done, the cylinder-efficiency and the thermodynamic efficiency cover all needs for identifying the engine's rank as a transformer of heat into useful energy.

As to capacity, there is no accepted form for the expression of the engine's rank relatively to other engines. A common one is to state the number of horse-power developed per ton of machinery. This definition usually includes the boilers and all accessories, and is very useful in marine engineering. But for the study of the science of engine-design it is too comprehensive. The number of pounds weight of actual engine per horse-power is also widely used. The only objection open against it as a characteristic of capacity is that it gives no idea as to either space or cost involved. In the writer's study of the gasengine problem he has needed to bring engines of the most diverse types into some comparison which would include all of these items in a way at least approximately just, and for that purpose has made use of what was defined as the builder's ratio in Chapter VIII, Part I. It 
was found to be of great service for this purpose; but the practice is not yet a matter of general acceptance.

Before entering upon a general discussion of the modification of theory in practice, however, it is necessary to acquire some familiarity with the machinery, so to speak, of engine-design, of the graphical methods whereby the actual engine-cylinder is brought into close touch with the purely theoretical diagrams which have been discussed hitherto. This can best be done by entering upon a given problem of engine-design.

Problem 34. Wanted: An engine to develop 100 horsepower under a boiler-pressure of IoO pounds by gage, when exhausting to the atmosphere and running at a speed of IOO revolutions per minute.

The choice of the items just specified might call for the exercise of judgment on the part of the designer or they might be determined for him by existing conditions. In either case, immediately the problem is entered upon, his judgment is called upon to decide upon what type of engine the design is to be founded.

The wisdom of this method of procedure needs more emphasis than mere passing mention. The chief characteristics in operation which determine the worth or the unworth of an engine are determined, not by the detail of design and construction which the engine embodies, but to the general type or class to which it belongs, and to the correspondence between the type chosen and the service to be performed. It is too widespread an idea that the successful design of a steam-plant consists in the discovery of the "best" engine, the "best" boiler, the "best" pump, etc. This is far from right. There is no literally best engine, etc. There are many better ones and many worse. There are some so bad that they would be worthless any- 
where; but there are none so good that they will not be worthless if located unwisely and set to perform work for which they were not designed. It is for the identification of the points in which engine and service should harmonize, and their recognition in practice, for which consideration must be turned to the question of the broad types of engine. If the wrong type be selected, for purchase or for design, no amount of skill in detail can produce good, or at any rate, the best, results.

The distinguishing characteristics between the several types of engine, excluding questions purely of form, such as vertical $v s$. horizontal, etc., depend almost entirely upon the class of valves and valve-gear with which each is provided. The study of the kinematics of valve-gears is no part of the work in hand; but the following analysis of their mechanical and thermodynamic effects is essential.

All cylinder-and-piston steam-engines come under classification into three distinct types, each of which has its plain characteristics :-

I. The single-valve fixed-eccentric type;

II. The four-valve fixed-eccentric type;

III. The single-valve variable-eccentric type.

Type I. This finds its typical illustration in the plain slide-valve throttle-governed engine, now almost obsolete for general power purposes. Its typical indicator-cards are shown in Fig. 34, the full line representing normal load, the dotted line overload, and the broken line light load. The resultant characteristics of the engine, when compared with those of the other types, may be stated relatively as follows :

I. Steam-distribution: All events fixed.

2. Efficiency: The poorest.

3. Capacity by unit of space or weight: The greatest.

4. Speed: Not limited by valve-gear; usually medium. 
5. Standard Ratio of expansion: I.2 to I.4.

6. Clearance: Maximum. ${ }^{1}$

7. Regulation: Poor.

8. Complexity: The least.

9. Cost: The least.

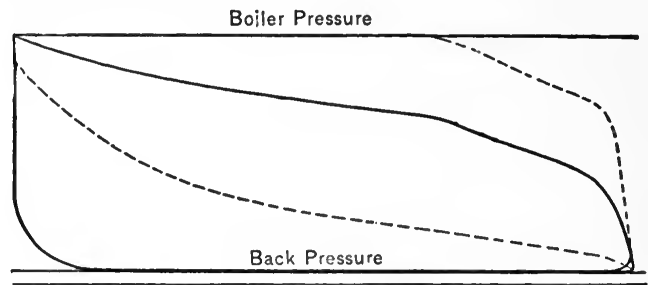

FIG. 34

It may sometimes be found modified in the following particulars, without effect upon its characteristics :-

(I) Piston-valve instead of slide-valve;

(2) Hand-control of speed instead of governor-control. It is now used chiefly for portable work, such as hoisting, pile-driving, etc., or in very small powers. Even for small powers, however, if the service be a continuous one, engines of Type III may better be substituted.

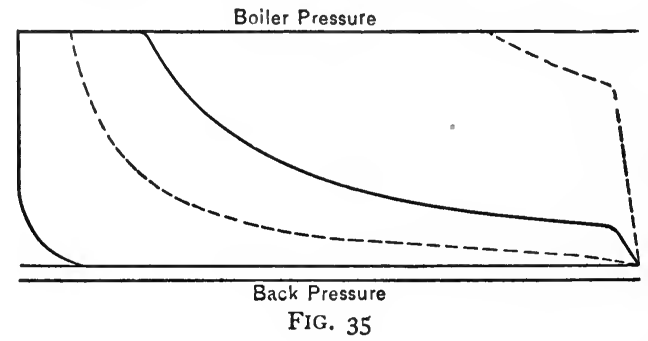

Type II. This finds its typical illustration in the standard Corliss engine, with centrifugal governor and drop cut-off gear. Its typical indicator-cards are shown in Fig. 35.

1 For definitions of "clearance" and "cylindrus" see page 205. 
The resultant characteristics, compared with those of the other types, are :-

Steam-distribution: Cut-off variable by governor; exhaust, compression, and admission fixed.

Efficiency: Above about Ioo horse-power, maximum; below about 100 horse-power, medium.

Capacity per unit of space or weight: Minimum.

Speed: Limited by valve-gear to about Ioo r.p.m.; usually less.

Standard Ratio of expansion: 5 to 6.

Clearance: Minimum.

Regulation: Poor to medium.

Complexity: Maximum.

Cost: Maximum.

This type is found under very wide modifications without effect upon its characteristics. Any engine in which the steam-distribution is as stated belongs to this class, although the valves may be slide-valves, as in the Greene, or poppet-valves, as in the Putnam, or gridiron-valves, as in the Wheelock, or Myers cut-off double-slides, as in the Buckeye. It may have two fixed eccentrics instead of one.

If the steam-valves, although Corliss in form, are actuated by positive connection to a variable eccentric in a shaftgovernor, as is now done in some designs of Corliss engines in order to permit high rotative speed, the engine belongs not to Type II but to

Type III, which finds its typical illustration in the highspeed, so-called " automatic," shaft-governor engine, usually fitted with a single piston-valve. Its typical diagrams are those of Fig. 36. The resultant characteristics, compared with those of the other types, are:-

Steam-distribution: All events varied with the cut-off by the governor. 
Efficiency: Above about roo horse-power, medium; below about 100 horse-power, maximum.

Capacity: Medium.

Speed: Not limited by valve-gear; usually high.

Standard Ratio of expansion: 4.

Clearance: Medium.

Regulation: The best.

Complexity: Medium.

Cost: Medium.

Amongst the possible modifications of this type the one of the first importance is that involved in the substitution of the Stephenson-link or similar reversing-gear in place of

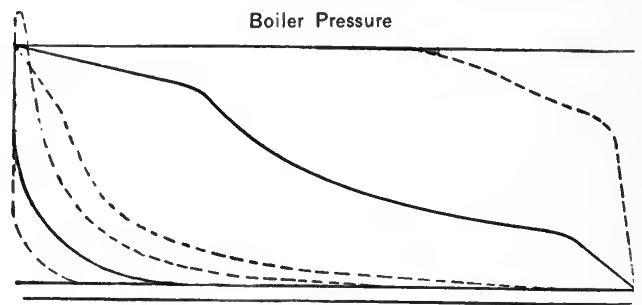

FIG. 36

the variable eccentric, for they are virtual equivalents. This brings all locomotive and all marine engines, except the old-fashioned walking-beam paddle-engine, into this class. Any engine having a slide-valve, balanced or unbalanced, single or double ported, or divided into two or four parts, if driven from a variable eccentric, comes within the same classification.

To return to Problem 34, it may be assumed that the engine is to belong to the Type II class, with a normal cut-off at one-fifth stroke. For while the text-book must emphasize the importance of selection according to typecharacteristics, it can give no rules for making a wise 
selection. That must depend upon judgment cultivated by experience.

The first duty is to construct a preliminary hypothetical indicator-card upon which to base estimates. This will, in the first instance, take the simple form $A B C D E$, Fig. 37. The pressure at $A B$ is 100 pounds by gage, or I I 4.5 absolute; that at $D E$ is atmospheric plus a proper allowance for back-pressure, say, 16 pounds absolute. The volumes are unknown; but $E D=5 A B$.

In the expansion of saturated steam in an engine-cylinder the supposed adiabatic is always modified by heatinterchanges with the cylinder-walls. These interchanges are quite complex, and vary widely in different cases. They will be discussed more elaborately at a later point in the text. Taken all together, however, under average conditions, they usually have the effect of bringing the expansioncurve away from a true adiabatic and into approximation with the equilateral hyperbola having the equation $\frac{P_{1}}{P_{2}}=\frac{V_{2}}{V_{1}}$. So, for the purposes in hand, it is always considered sufficiently exact (and it is certainly very convenient) to adopt this curve as being the same as $B C$ of Fig. 37.

N.B. This equation is identical with that for the isothermal curve with air or other permanent gas as the working-fuid. But this fact must not lead to confusion with the conditions which prevail when steam is the working-fluid. The above equation does not represent an isothermal with steam, as is often erroneously stated. The isothermals in the steam-engine cycle are the lines $A B$ and $D E$, and their equation is $P=$ a constant.

For the drawing of the equilateral hyperbola $B C$, Fig. 37, there is a very convenient graphical method. Although the data of the problem under discussion do not give any direct information as to volumes, and it is therefore impossible to draw the curve to scale, at this point in the development of the problem, yet it will be convenient to have 
before the eye an illustrative curve of indefinite scale upon which to base argument. Taking any initial volume at the stated boiler-pressure, as at the point $B$, the remainder of the curve may be determined in this way:-

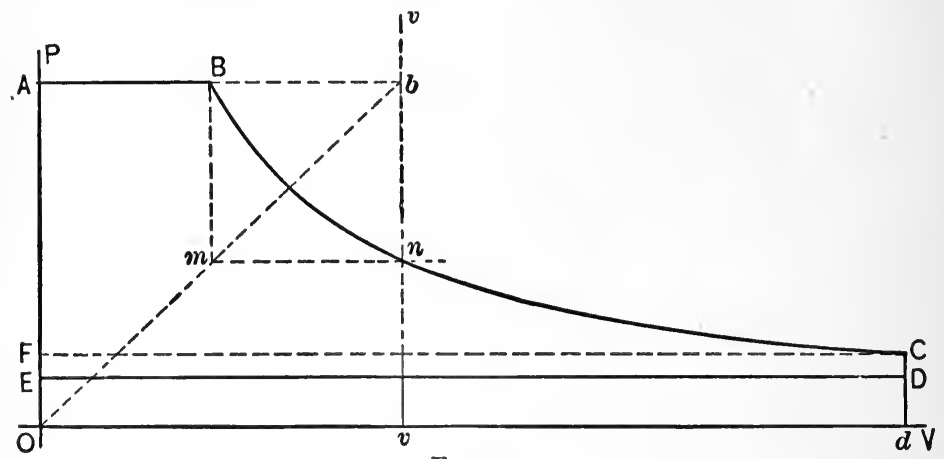

FIG. 37

Let it be desired to know the pressure which will prevail at some other volume than the initial, such as at $v v$.

(I) Draw the constant-pressure line $B b$ until it intersects $v v$ at $b$;

(2) From $b$ draw a diagonal $b 0$ to the absolute zeropoint of pressure and volume ;

(3) From $B$ draw a constant-volume line $B m$ until it intersects $b O$ at $m$;

(4) From $m$ draw a constant-pressure line $m n$ until it intersects $v v$ at $n$. $n$ is the desired point through which the curve is to be drawn.

For finding an unknown point at any desired pressure other than the original, interchange all the italicized words "pressure" and "volume" in the above. Volumes or pressures may be greater or less than the original without altering the rule to be followed.

The area of the cycle of Fig. 37 may be conveniently divided into two portions: $A B C F$ and FCDE. Let the 
volume at $C=V_{C}$. Then area $A B C F=P_{B} V_{B} \log _{e} r=$ $($ I $14.5 \times$ I 44$) \times \frac{V_{C}}{5} \times \log _{e} 5$. The area $F C D E=F C \times C D$ $=V_{C}\left[\left(\frac{1}{5} \times \mathrm{I} 14.5\right)-\mathrm{I} 6\right] \times \mathrm{I} 44=6.9 \times V_{C} \times \mathrm{I} 44$. Since the engine is to develop Ioo horse-power at Ioo revolutions, or 200 strokes, per minute the work necessarily developed per stroke, in foot-pounds, must be

$$
W=\frac{100 \times 33000}{200}=\mathrm{I} 6,500 \text { foot-pounds. }
$$

Hence $\left(\mathrm{II} 4.5 \times \mathrm{I} 44 \times \frac{1}{5} V_{C} \times \mathrm{I} .609\right)+\left(6.9 \times \mathrm{I} 44 \times V_{C}\right)$

$$
=6300 V_{C}=\mathrm{I} 6,500 \text {, }
$$

or

$$
V_{C}=2.620 \text { cubic feet. }
$$

This result is of no immediate value, because no allowance has been made for the effect of clearance and compression. In professional engineering offices this allowance is usually made by judgment, as a percentage in addition to the calculated volume; and since the result desired is nothing more accurate than the general dimensions of a cylinder of a capacity which is entirely nominal, this is usually amply accurate. But where the necessary experience as a basis for estimate is lacking, clearance must be allowed for in the following way. In compound engines, too, where it is desired that a certain effective cylindervolume be attained in order to establish a certain effective cylinder-ratio, it is important that the effect of clearance be carefully considered.

Let the probable clearance to be expected in the cylinder when completed be estimated at 2 per cent. Suppose that it be the designer's judgment that this clearance should be filled with steam of two-thirds boiler-pressure at the completion of compression. The situation is shown in Fig. 38. For the moment call the volume of the cylindrus, or $f C$, 
equal to unity. Then $F f=0.02$ and $F C=$ 1.02. $A B$ must equal 0.204 , in order to maintain a ratio of expansion of 5 . Since the $V_{C}$ of Fig. 38 is equal to I.02 times $f C$, it may be stated, from Fig. 37 , that the area $a B C D E$ of Fig. 38 is

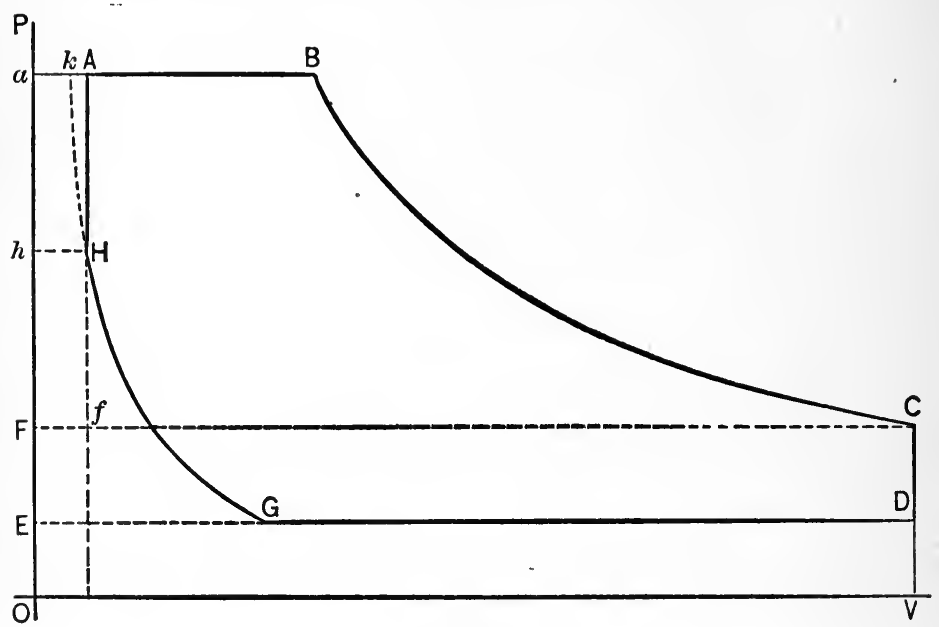

FIG. 38

equal to $\mathrm{I} .02 \times 6300 f C=6426 f C$. The area $h H G E$ must be equal to $P_{H} V_{H} \log _{e}\left(P_{H} \div P_{G}\right)$

$$
=\left(\frac{2}{3} \mathrm{II} 4.5 \times \mathrm{I} 44\right) 0.02 f C \log _{e}\left(\frac{2}{3} \times \frac{\mathrm{II} 4.5}{\mathrm{I} 6}\right)=343 f C .
$$

The area $a A H h=\frac{1}{3} \times 1 \mathrm{I} 4.5 \times 144 \times 0.02 f C=$ I $10 f C$. Therefore the area $A B C D G H A=(6426-343-$ I Io $) f C$ $=5973 \mathrm{fC}$. Therefore, by the premises of the problem, $5973 f C=\mathrm{I} 6,500 \mathrm{ft}$.lbs.; whence $f C=2.76$ cubic feet.

Piston-speed. - This volume can of course be arranged in any form that the designer prefers : a long narrow cylinder or a short and wide one. This would ordinarily be determined by the piston-speed, which is arbitrarily defined as the total distance travelled by the piston in feet per 
minute. It is usually considered desirable to keep this speed between 400 and 800 feet per minute, the larger figure for large engines and the smaller one for the little ones. In the present problem a piston-speed of 450 feet per minute would give a stroke of 27 inches and a diameter of 15 inches, in order to displace 2.76 cubic feet per stroke. In practice the stroke would probably be made 28 inches, rather than 27.

Mean Effective Pressure. - The mean effective pressure in an engine-cylinder is defined as that pressure which, if evenly maintained throughout the entire stroke, would accom plish the same work as the actual varying pressure.

The writer's experience has gone to show that for the ordinary purposes of a preliminary estimate, lumping all the questions as to probable back-pressure, cushion, and clearance to be incurred under a single coefficient based upon the type of engine, the following formulæ are sufficiently accurate and are much quicker to handle than the plan just described. If $P$ be the given boiler-pressure (by gage) then the mean effective pressure, $p$, can be had as follows : ${ }^{1}$ -

For Type I: $p=0.7 P-6$; or $p=\frac{3}{4}(P+15)-20$. For Type II: $p=0.4 P-2$; or $p=\frac{1}{2}(P+15)-17$. For Type III : $p=0.6 P-8$; or $p=\frac{2}{3}(P+15)-23$.

The second set of formulæ are somewhat more accurate and also more cumbrous than the first set. Since accuracy in such a case is a purely comparative term it is proper to add that the first set becomes unsatisfactory only when extreme conditions of low boiler-pressure prevail or when a vacuum is used.

1 These formulæ are also useful for estimating the proper capacity of an engine the records of which are wanting. 
Application of the first formula for Type II to the present problem gives $V_{C}=3.015$, or a cylinder measuring $15^{\prime \prime} \times 30^{\prime \prime}$, which is on the safe side of the more exact figure determined above. The second formula gives a cylinder $15^{\prime \prime} \times 28^{\prime \prime}$; which agrees exactly with the dimensions calculated above. At pressures lower than 100 by gage, which is unusually high for a simple engine, the agreement between the results from the empirical and the exact formulæ would be closer.

With this preliminary outline of the relations between pressure-volume curves and the actual cylinder, attention may be intelligently returned to the thermodynamics of engine-action. The discussion of the steam-engine cycle, which was left in its purely theoretic aspect, may now be carried on into its actual aspect in practice.

The Effect of the Cylinder-walls upon Efficiency. - The Rankine cycle, the cycle of the perfect steam-engine, is impossible of reproduction in practice. The suppositions upon which its theory is founded are :-

(I) No heat-interchange between the steam and the surrounding walls;

(2) Complete expansion to the lowest available pressure and temperature;

(3) Instantaneous action of valves ;

(4) No leakage past valves or piston; and

(5) No clearance.

None of these conditions is realizable in practice except the second, and that is seldom desirable for practical reasons. (See pages 234-235.)

Condensation and Reëvaporation. - Of the five sources of variation of the actual cycle from the theoretic, the first is by far the most important in its effects upon efficiency. It may be remembered that the work which placed the 
name of Watt at the head of the list of steam-engineers was the study of this phenomenon. Impressed by the large amount of steam consumed by a small model engine submitted to him for repair, he attained its explanation by the following series of determinations:-

I. The proof beyond doubt that a greater volume of steam entered the cylinder at each stroke than the cylinder could possibly contain. Since the only possible method of measuring the quantity of steam entering the cylinder at each stroke was in terms of its weight, which could be measured before the water entered the boiler, Watt's task involved the determination of the specific volume of steam. His first approximation gave I : I 800 as the ratio between volumes of a given weight of water when liquid and when vapor. This led to the rule-of-thumb, current for long afterward among steam-engineers: "A cubic inch of water makes a cubic foot of steam," which implies a ratio of I : I 728. This, of course, was all based upon steam of atmospheric pressure. We now know that the true ratio is about $\mathrm{I}: \mathrm{I} 660$. Knowing the weight of water evaporated in the boiler for each stroke of the engine, Watt knew from this ratio its volume. He was surprised to find it some four to six times that of the cylinder itself. In other words, the cylinder-condensation amounted to from 75 to $83 \%$.

2. Watt proved that this condensation was not due to radiation of heat from the external surface of the cylinder. More accurately, these proofs were reversed in order; that is, suspecting condensation from general considerations, and radiation being the readiest explanation of condensation, Watt first proved that the prevention of radiation was no remedy for the trouble. As this result would lead the ordinary mind to the conclusion that there could not have been any condensation, it is significant of Watt's 
genius that at this point in his investigations he persisted in the difficult task of proving conclusively that condensation did take place, and to an enormous amount. If the cylinder-walls did not take up the heat released by this condensation and radiate it, what could pick it up and carry it away? The only other thing in the cylinder besides the metal was water, and this hint led him to his next step.

3. The determination of the latent and specific heats of steam and water, which showed that even a slight portion of the water injected into the cylinder at each stroke for the purpose of condensation was quite able to account for the trouble. To prevent it, therefore, could obviously be prescribed Watt's famous rule, easily deduced from what had preceded :-

4. "Keep the cylinder dry, and as hot as the entering steam." To accomplish the first, one must use a separate condenser. For the second was needed the steam-jacket. Both, but especially the first, have survived until the present day as the first essentials to steam-engine economy.

It was but a slight step in advance of this to recognize that the cylinder-walls as well as the water upon them were active in losing heat from the entering steam to the exhaust. It had been proven beyond doubt that it was not loss of heat through the walls to the air, but the alternating passage of heat from steam to metal and water, and then back again from metal to steam, which lay back of the greatest loss of efficiency in the steam-engine. This is still the fact to-day, in spite of the century of effort at minimizing the thermal action under discussion. Its determination at that early date classed Watt as a scientist as well as a skilled mechanic, and in both fields of activity a genius.

When boiler-steam enters an engine-cylinder, at or just before dead-centre is reached, it finds itself within a disk- 
shaped space enclosed by the cylinder-head, the piston and a narrow strip of cylindrical barrel-surface. This space is also one with that of the ports and passages for admission and exhaust of steam. In both its portions this steam-filled cavity presents a very great proportion of surface of surrounding wall relatively to its volume. This surface, too, for reasons which have already been outlined and which are to be elaborated later in this discussion, is always at a temperature much below that of the entering steam. The transfer of heat from a moist vapor to iron is very rapid. Therefore considerable steam condenses upon the surface of the metal, at the same time heating the latter to approximately its own temperature to a certain depth, usually a very small fraction of the thickness of the walls.

The steam thus condensed into water deposits upon the surface of the metal in bead-like or sweat-like drops. Since the transfer of heat between water and iron, or between steam and water, is still more rapid than that between steam and iron or between iron and iron, the presence of this water upon the surface of the metal very much exaggerates the phenomenon just described. In fact, in many of these cylinder-phenomena the water adhering to the cylinder-walls is much more active as a thermal agent than is the iron, which is to be explained by the fact that the specific heat of water is much higher than that of iron.

It is to be especially noted that the greater portion of all of the surface which is at any time brought to steamtemperature is exposed to the steam before the piston moves out upon its stroke, and this for a comparatively long period of time. This means that the bulk of the condensation occurring in the cylinder does so while the engine is on dead-centre. It is this fact which primarily accounts for the need of lead in a steam-engine valve-gear. Something like 20 to $40 \%$ of all the steam used is wanted 
before the stroke begins. The one other basis for lead the need for having the valve appreciably open when the piston starts on its stroke with accelerating velocity - is entirely a secondary one ; for in every case the valve-opening would be accelerated more rapidly than, and would attain a maximum before, the velocity of the piston does the same, even if there were no lead.

As the piston moves out upon its stroke more and more cylinder-surface is exposed to boiler-temperature. Condensation continues, but at a much reduced rate. When cut-off takes place the piston is usually moving quite rapidly and the exposed surface is increasing correspondingly.

As soon as cut-off is effected, however, expansion ensues and the temperature of the steam-and-water mixture within the cylinder necessarily drops with the pressure. The bulk of the metal in the cylinder-walls, too, is not at the temperature of the atmosphere, but at some mean between that and boiler-temperature, determined by the equilibrium which is established between the various gains and losses of heat without and within. The temperature-difference between the steam within the cylinder and the fresh surface uncovered by the motion of the piston rapidly decreases, therefore, after cut-off takes place, and with it decreases the transfer of heat between the two. The rate of condensation, which had steadily decreased as the piston advanced, soon becomes zero. At this point in the stroke the proportion of water to total weight of steam and water within the cylinder may be anywhere from io to $60 \%$, according to the size of cylinder and conditions of operation. At cut-off it is seldom more than 5 or $10 \%$ less than this.

The temperature of the expanding steam has no sooner fallen to the mean temperature of the iron than further expansion, or the opening of the exhaust-valve, carries it be- 
low. The temperature-difference between the two becomes reversed, the direction of heat-transfer is reversed, and a reëvaporation of the moisture deposited upon the cylinderwalls takes the place of the previous condensation. This continues, being very much accelerated by the opening of the exhaust-valve and the consequent reduction of pressure upon the hot water, until the latter is completely evaporated and the walls are dry. After that the rate of heat-transfer between metal and steam is reduced to a very small fraction - something like one two-hundredth, according to some authorities - of what it was while the walls were wet.

The reëvaporation of the water from the cylinder-walls of course demands heat for its performance, and the abstraction of this heat from the metal lowers the temperature of its inner surface practically to that of the exhaust-steam. It is this abstraction of heat by reëvaporation, and not the loss of heat to the outer air by external radiation or conduction, which makes the demand for initial condensation upon the next admission of steam. Comparing these two sources of dissipation of heat: radiation and reëvaporation, the former amounts to not over I to ro\% of the latter. It is usually about $3 \%$ in single-cylinder non-jacketed engines.

\section{The Entropy-temperature Analysis}

This double process, condensation and reëvaporation, is the first and greatest cause of the departure of actual steamengine efficiency from the maximum possible determined by the external conditions (such as boiler-pressure, vacuum, etc.) under which the engine is forced to operate. The reduction of this loss has been the chief anxiety of the steam-engine designer since the days of Watt, and the amount of its reduction has been one of the best measures of progress accomplished. The effect of purely external 
conditions upon engine-efficiency is so well understood, the comparative cost and gain to be associated with a given rise of steam-pressure or decrease of back-pressure are so patent to the ordinary non-scientific designer, that their discussion, albeit important and demanding experience and judgment, is quite devoid of mystery. With the internal factors of engine-efficiency, on the other hand, although identified in part as early as the time of Watt and pursued ever since, the difficulty of prediction before construction, or of measurement afterward, has prevented the profession from yet accumulating sufficient data for a satisfactory science of steam-engine design. The amount of condensation and reëvaporation occurring in an engine is the resultant of a great many variables, all of which differ in each engine from those of any previous one with which experience has been had. The building of a series of engines, in each successive one of which only one variable shall have been altered in comparison with the preceding one, is a task utterly beyond the limitations of engineering possibility, chiefly by reason of cost, but also on account of the indefinite length of the series needed for accomplishing a definite commercial result. It is therefore of the utmost importance to reduce the study of condensation and reëvaporation, and of the other points in which the actual cycle departs from the theoretic, to as exact an analysis of cause and effect as possible. It is equally important that the adopted method of doing this be applicable to every single engine-test, without necessity for using two tests in order to get one reading, and that a merely comparative one.

Such an analysis is quite possible, and demands for its performance, besides the modicum of patient labor always essential to an analysis of anything, only the following data :- 
(I) The dimensions of the cylinders, including percentages of clearance and diameters of rods;

(2) The speed, which should be known to a fraction of a revolution per minute, by counting over a period of several minutes;

(3) The indicated horse-power, from cards taken under conditions, as to boiler-pressure, cut-off, etc., as nearly constant as possible;

(4) The amount of water (steam) passing through the cylinder per horse-power or per unit of time; and

(5) That the leakage of valves and pistons has been brought to the minimum possible.

These points are all known as the result of an ordinary engine-test.

At the start the following distinctions should be gotten clearly in mind.

Cylindrus and Clearance. - At every stroke of the piston a certain volume is displaced, equal to the effective area of the piston times the length of the stroke. This effective area may be the area of the piston itself, or there may be the cross-sectional area of a piston-rod, a tail-rod or a trunk to be deducted. The volume thus displaced is known as the cylindrus.

At the completion of each stroke there remains in the cylinder, beyond the piston in the direction of its late motion and between the piston and cylinder-head, a certain undisplaced volume, which includes all ports and passages back to the lips of the valves which enclose this space. This volume is known as the clearance. It is always measured and expressed as a percentage of the cylindrus.

The total volume in the cylinder at any point of the stroke always consists of the sum of the clearance and a portion of the cylindrus. 
Cylinder-feed and Cushion-steam. - At each stroke of the engine there enters the cylinder a certain quantity of steam from the boiler. This steam may go to fill the clearance-space, it may condense in warming the cylinderwalls, it may go to propel the piston. Whatever its destination it has to be supplied by the boiler. It is known as the cylinder-feed. It is always measured in pounds avoirdupois.

At the beginning of each stroke, when the admissionvalve opens, the entering steam finds the cylinder already occupied by a certain quantity of steam. This is the steam which has been imprisoned in the end of the cylinder by the closing of the exhaust-valve before the previous exhaust-stroke was completed. This steam is known as the cushion-steam, from the frequent reliance upon its elastic compressibility for arresting the motion of the piston at the end of the stroke without undue shock upon the crank and connecting-rod. It is always measured in terms of the weight of cylinder-feed passing through the engine at each stroke, and is stated as a proportion of one pound of the latter, as, for instance: "Twenty-three hundredths of a pound per pound of cylinder-feed." This ratio may be determined on the basis of a single stroke, or of a unit of time. It is usually easiest to measure the cushion-steam for a single stroke and the cylinder-feed for an hour; the two are then easily reduced to the same terms by means of the known engine-speed.

This cushion-steam should always be considered as a part of the engine's mechanism. If the cylinder-walls and the cylinder-feed were to refrain from thermal interference with it, it would give out in expansion during the workingstroke of the engine just as much work as it absorbed during compression. In this respect it would be the exact equivalent of a steel spring of perfect resilience. Nor does 
this statement ignore the widely discussed question of the value of clearance and cushioning. The truth of it is not affected by the fact that the piston may not receive the work thus returned during expansion, because the exhaustvalve may be open or because the cylinder-walls never do refrain from thermal interference. Nor by the fact that, in some types of engines, the point in the stroke at which the exhaust-valve closes varies under varying load, and so varies the amount of steam trapped in the cylinder from moment to moment. All of these questions are quite secondary and incidental to the main one, viz. : That in every given stroke, or complete cycle, of an engine there is a portion of the steam present which does not enter with the entering steam, which does not leave with the exhauststeam, and which cannot be regarded as a means of heatsupply to the engine. Moreover, this cushion-steam, unless it can be imagined as capable of storing up or supplying heat indefinitely, must have had balanced up to a net zero for it by the cylinder-feed, by the end of each cycle, all the losses or gains of heat whatever which it may have made at earlier points of the cycle. This must hold true whether the gains or losses went to the cylinder-feed itself, to the outside air, to work, or to aught else. Such is the clearinghouse character of the cushion-steam.

For the cylinder-feed alone can bring heat into the engine. It alone, therefore, is chargeable with all losses of heat from the engine, whether they took place from it directly or indirectly, whether they be desirable losses (as in the form of work) or undesirable ones.

It is to be repeated, then, that the first object of a rational analysis of efficiency is to charge up all losses against the cylinder-feed. This can be done only through a knowledge of its volume and pressure at each instant of its existence in the engine. If, however, we turn to the steam-engine 
indicator as a source of light upon this question, we are confronted with the immediate difficulty: The indicator reveals volumes and pressures of cylindrus, not of cylinderfeed. It is the first duty, then, to translate one into the other.

Let $v$ equal the total volume existing within the cylinder at any point of the piston's motion. It is given by the abscissæ of the indicator-card, when measured from a vertical axis placed at the proper distance from the end to represent the percentage of clearance.

Let $c$ equal the clearance-volume, a constant.

Let $z$ equal the portion of the cylindrus which has been developed at the same point of the piston's motion. It is measured by the abscissæ of the indicator-card.

Let $y$ equal the volume of cushion-steam at the same instant.

Let $x$ equal the volume of cylinder-feed at the same instant.

Then

$$
v=c+z
$$

and

$$
v=y+x \text {. }
$$

Whence

$$
x=z+c-y .
$$

In other words, from the known variable volume of cylindrus, $z$, the unknown variable volume of cylinder-feed, $x$, may be known by first adding the constant volume of clearance, $c$, and then subtracting the variable volume of cushion-steam, $y$.

Pressures, since all the volumes under discussion are freely open to another, must be the same in all the portions of steam at any given instant, and may be read from the indicator without translation.

Further consideration of the above proposition regarding volumes raises the following question: Since the proportion of cushion-steam to cylinder-feed is the same through- 
out a given stroke, and since these two quantities of steam expand and compress together, in intimate mixture one with the other, why must not the volume of each vary in equal proportion as the pressure changes? Physically this must be the case. The entire body of steam within the cylinder must be in practically the same condition, except under unusual disturbances. But analytically and mathematically a marked distinction must be drawn. Since the cushion-steam cannot of itself constitute a source of heat, any loss of heat which it experiences at any given moment must be imagined as immediately made good by an equivalent supply of heat to it from the cylinder-feed. Conversely, any gain of heat which it may experience must be regarded as immediately turned over to the cylinder-feed, for the latter alone can carry heat out of the engine. In other words, the cushion-steam must always be regarded as expanding and compressing adiabatically. All losses and gains of heat, although actually experienced by either or both of the two steam-quantities, are to be charged against the cylinder-feed alone. This gives the volume of the cushion-steam as a known variable at any point, deducible from the known pressure at that point. This makes all of the terms of the right-hand member of Equation I03 known quantities.

The process indicated in Equation IO3 is most easily performed graphically, especially as the quantities determined by the indicator come to us as graphical measurements. To facilitate this work, together with some other mathematical deductions needing to be drawn, the author has projected a large blank diagram fit for rapid work upon the drafting-board upon a scale so large as to minimize both graphical error and strain upon the eye. ${ }^{1}$ There is nothing about it, however, which cannot be readily produced by

1 Published by Progressive Age Pub. Co., 280 Broadway, New York City. 
oneself with the aid of a steam-table, though at considerable cost of time and trouble. Its essentials are represented diagrammatically in Fig. 39.

The sheet is divided into four portions, or quadrants, by one vertical and one horizontal axis meeting in the centre of the sheet at $O$. The right-hand horizontal axis, from $O$ as a zero-point, measures volumes, the scale being plainly

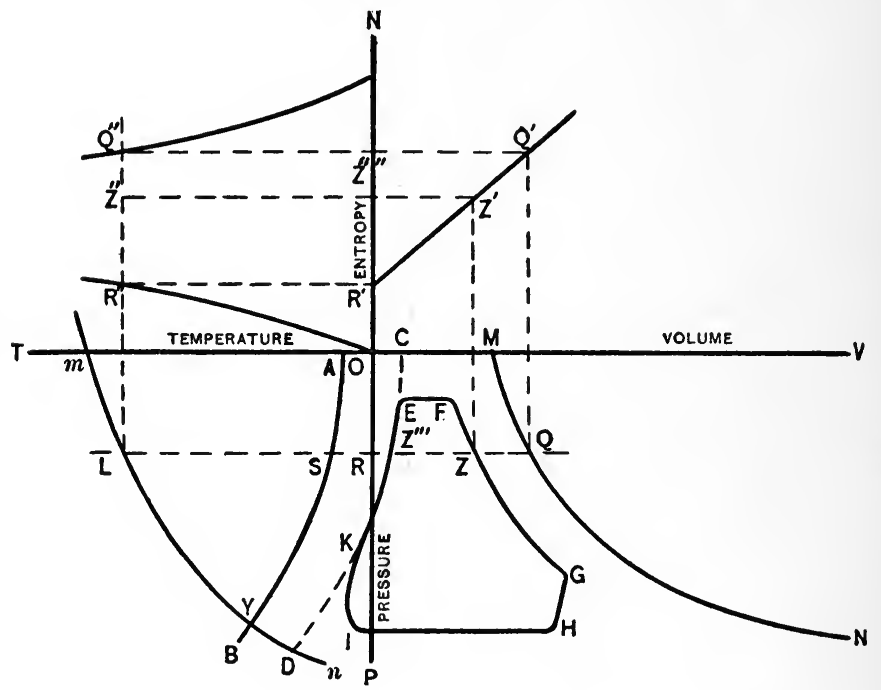

FIG. 39

indicated. The lower vertical axis similarly measures pressures, except that the zero-point is at the edge of the paper. The left-hand horizontal axis measures temperatures, the zero-point being at $O$, which represents the arbitrary zero of the steam-tables, the melting-point of ice, or $32^{\circ} \mathrm{F}$. The upper vertical axis measures entropies from a zero-point at $O$, which also assumes the arbitrary zero of the steam-tables.

From this arrangement it is obvious that the lower right- 
hand quadrant may be used to indicate the relations between pressure and volume, as upon the ordinary indicator-card. The lower left-hand quadrant will display relations between temperature and pressure; it is also used, however, as a prolongation of the pressure-volume quadrant into a field of negative volumes, which are measured horizontally to the left from $O$ on the same scale as positive volumes are measured from $O$ to the right. The upper right-hand quadrant will display relations between volume and entropy. The upper left-hand quadrant will display relations between temperature and entropy, but in a position in reference to the eye rotated ninety degrees from that adopted in the previous chapters.

It is in the premises of the diagram that these several axes and fields of coördinates are to be used to reveal the condition of the cylinder-feed within the engine during any given stroke. It is supposed that from the data listed on page 205 (this chapter) the cylindrus and the clearance have been calculated in cubic feet, that the weight of steam passing through the engine in pounds per hour is known, and that the engine has run under conditions so nearly constant that either a selected actual indicator-card, or an artificial one constructed from the actual ones on a wise system of averages, may be taken as accurately representing the change through which the cylinder-steam passes as the stroke progresses. Further, as a matter of convenience solely, the unit of time upon which all calculations are to be based is that required for the passage of one pound of cylinder-feed through the engine. But this unit of time is most conveniently expressed in number of engine-strokes rather than in number of seconds, minutes, etc. Any other unit of time, such as the hour, minute, second, or the single stroke, might be used with equal propriety, but at much greater labor of calculation. To determine this unit 
of time the number of working-strokes per hour need merely be divided by the number of pounds of cylinderfeed per hour. It is obvious that the result will depend largely upon the size of the engine, amongst other factors. It may be almost any figure, from a quite large number for very small engines to a fraction of unity for very large ones.

The volume-scale of the indicator-card which fits properly with the above assumptions is given by the fact that the total length of the card must represent the cylindrusvolume evolved during the chosen unit of time. This is plainly the actual cylindrus multiplied by the basic number of strokes. This is the basic volumetric scale of the indicator-card, and all other measurements taken from the card must be read to this same scale. ${ }^{1}$

Next, let the clearance, known as a percentage of the cylindrus, be laid off from the admission-end of the indicator-card as this same percentage of its total length, and through the point thus obtained let a vertical zero-axis of volumes be raised normal to the atmospheric line. If now any point of the card be measured as to horizontal distance from this axis, on the proper scale already obtained, the reading will be cubic feet of total cylinder-volume per pound of cylinder-feed passing the engine.

In order to know the volumetric condition of the cylinderfeed, it now remains to subtract the variable volume of the cushion-steam. One of the values of this variable may be known from the indicator-card; the others must be deduced from the first.

${ }^{1}$ This ratio or proportion - for it could hardly be called a scale - may be reduced to a comparatively convenient scale by measuring the length of the indicator-card in sixteenths, thirty-seconds, or hundredths of an inch, or in millimeters, according to what scales are at hand, and then dividing the above total cylindrus-volume per unit of time by the same. This gives a scale reading "so many cubic feet per sixteenth inch, or per millimeter," etc. 
Thus, it is known empirically from steam-engine practice that, except in extreme cases only, reëvaporation is always completed early in the exhaust-stroke. This insures that the steam in the cylinder at the beginning of compression is dry saturated steam, and hence its volume and pressure determine its quantity. Even in steamjacketed cylinders, where the tendency would be to superheat the steam in the cylinder immediately reëvaporation were complete, the heat-transfer to the dry steam is so slow that the departure of the truth from this assumption is insignificant.

Immediately the exhaust-valve is closed, however, two forces begin to alter the condition of the steam:

( I) The thermodynamic supply of energy to the steam by compression tends to superheat it (see page 92, point $e$ ).

(2) The rise of temperature due to compression tends to conduct heat from steam to cylinder-walls, which now begin to present a very large surface to the comparatively small volume of steam.

In nearly every actual case the second process is much the stronger of the two, and the cushion-steam steadily becomes more moist as compression proceeds. It is not

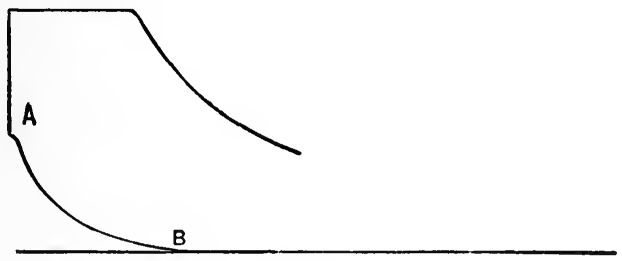

FIG. 40

uncommon to find an engine in which this is so far true that the compression finally attains a degree where the small plate-like volume of cushion-steam loses its heat so rapidly to the enveloping piston-face and cylinder-cover 
that it condenses into water isothermally as the piston completes the last portion of its stroke. The indicatorcard in such cases shows a compression-curve similar to Fig. 40, the isothermal condensation showing at $A$. It is therefore probable that all the steam in the cylinder is dry saturated immediately after exhaust-closure, and even in those cases where a light superheat is suspected the second of the above two forces operates to negative this source of error. ${ }^{1}$ If, therefore, a point on the card, such

${ }^{1}$ As this assumption of a condition of dry saturation at this point is a basic one, affecting all the subsequent work, it is proper to investigate not only its truth, which is difficult to do, but also the proportionate effect of its error upon the final results. To do this let it be assumed that the pressure-range of compression in the actual engine seldom exceeds that from 16 to 100 pounds absolute in a simple non-condensing engine, or an equivalent ratio for condensing engines or for cylinders exhausting into receivers. Under such conditions, therefore, let the resultant final volume be compared under four suppositions as to initial condition: (1) Dry saturation; (2) $10 \%$ wet; (3) $20 \%$ wet; (4) $50^{\circ}$ of superheat. Any engine which did not fall within these limits would be so abnormal in all of its activities as to either be unworthy of a test at all or else worthy of especial investigation.

The initial volumes of these four conditions may be stated relatively by calling the first 100. Then (2) must be 90.68; (3) 81.36; and (4) about 107.39. After adiabatic compression to roo pounds absolute the final volumes of the three would be: (1) 24.02 ; (2) 18.04 ; (3) 15.67 ; (4) 25.78 .

If it be assumed that the reduction of volume for all conditions is the same as that for dry saturation at the start, the four results are: (1) 24.02; (2) 21.78; (3) 19.53 ; (4) 25.80 . Numbers (2) and (3) are over $20 \%$ too large; (4) is practically correct. If a similar assumption be applied to steam of original condition of (2), the four results are: (1) 19.85 ; (2) 18.04 ; (3) 16.14 ; (4) 21.34. Now (I) and (4) are some $17 \%$ too small; (3) is within $3 \%$ of correct.

When it is considered that these assumed conditions are extreme and that the weight of cushion-steam rarely exceeds one-fourth of the cylinder-feed (which would reduce these percentages of error, when compared with the whole quantity of steam in the cylinder, to one-fifth of the figures stated), good basis is seen for the conclusions: (I) An assumption of dry saturation at the beginning of compression leads to no appreciable error in results if the steam be actually dry or superheated at start, and to an error of from I $\%$ to $5 \%$ if 
as $B$, be chosen, which represents an instant when the exhaust-valve is known to have been only recently completely closed, and if the volume and pressure at $B$ be measured by the scales already described, they will reveal the quantity of cushion-steam present at the instant per one pound of cylinder-feed.

Let these measurements be laid off on the diagram at the point $Y$, Fig. 39, the volume being regarded as a negative one. Through $Y$ let an adiabatic $A B$ be constructed. It will represent the volumes which the cushion-steam ought to occupy for every given pressure in the cylinder. In the published blank diagram will be found, in this portion of lower left-hand quadrant, a number of such adiabatics. With them as guides, when once the point $Y$ is determined, the desired adiabatic may be laid off by eye. These adiabatics are the pressure-volume ones, for the several weights noted on the curves themselves, corresponding to the single entropy-temperature adiabatic shown in the entropy-temperature quadrant. All agree in being based upon an original dry-steam point at an absolute pressure of 41.56 pounds per square inch, at which pressure the volume of one pound is ro cubic feet. Should the original observed point $Y$ happen to fall at this pressure, the weight of cushion-steam present may be read directly from the weights pointed on the several curves. If not, however, as would usually be the case, the weight as given by the curves need only be multiplied by the percentage indicated in the list of them to be found in the extreme lower left-hand corner of the sheet, the proper one

the steam be quite wet at the start; (2) Assumption of moisture at the start leads to no appreciable error if the steam actually be as moist as assumed or even much more so, and to an error of from $1 \%$ to $3 \%$ if it actually be drier than assumed. The actual error, under average conditions, will not exceed one-third of these figures. 
being chosen according to the pressure-level of the point $Y$, in order to give the actual weight of cushion-steam present per pound of cylinder-feed. A knowledge of this weight is not needed, however, for any further process of this analysis, but may be desired for other reasons.

In the straight vertical axis which was drawn outside the clearance on the indicator-card is to be had a zero-axis of total cylinder-volumes, as already noted. The object in view is to deduct from those volumes the variable volumes of cushion-steam in order to find the variable volumes of cylinder-feed. If, now, the line $A B$, Fig. 39 , be used as a curvilinear zero-axis of total cylinder-volumes, and if from it any measurement taken from the indicator-card, such as $S Z$ (translated into the proper scale), be laid off to the right, then the axis $O P$ must automatically cut off from it the volume $S R$ which should be properly occupied by the cushion-steam at this pressure. The remainder $R Z$ measures the volume which the cylinder-feed would have were all the heat-losses and gains charged against it. $O P$ therefore becomes the zero-axis of cylinder-feed volumes, and the lower right-hand quadrant becomes a rectangular coördinate field revealing the pressure-volume conditions of one pound of cylinder-feed.

Q.E.F.

The indicator-card should now be reproduced, point by point, in the above manner. It will appear, distorted in form, something like the curve EFZGHIK, Fig. 39. (By handling the points in horizontal pairs, as $Z$ and $Z^{\prime \prime \prime}$, much labor can be saved.) In this form it is of no immediate value, but from it can easily be deduced the entropy-temperature changes incurred by the cylinder-feed, and they, in turn, will show plainly just when and in what amount heat was lost or gained.

Let attention be confined to the illustrative point $Z$.

So long as the distorted indicator-card lies between the 
curves $A B$ and $M N$ the assurance is had that the cylinder is filled with saturated steam more or less moist; for the curve $M N$ is drawn as a "saturation-curve," representing the relation between volume and pressure of dry saturated steam; or, in other words, $M N$ is one of the columns of the steam-table represented graphically. In the temperature-pressure quadrant is a similar curve, $m n$, showing the relation between the temperature and pressure of dry saturated steam. Therefore if a line be run horizontally across from $Z$ until it meets $m n$, at $L$, and thence a vertical line be run to $R^{\prime \prime}$ and $Q^{\prime \prime}$, it is certain that the entropy-temperature condition of the steam at the point $Z$ must be shown at some point, such as $Z^{\prime \prime}$, on the line $R^{\prime \prime} Q^{\prime \prime}$. Which is the proper point, however, can be known only through information as to volumes.

If a horizontal be run from $R^{\prime \prime}$ to the vertical axis at $R^{\prime}$, the latter point must represent the relation of entropy to volume for water at the pressure shown by $Z$; for the volume of one pound of water is so small as to be invisible on the scale chosen. The volume of one pound of $d r y$ steam, however, of the pressure shown at $Z$ is shown by the point $Q$, and may be carried up into the entropy-volume quadrant by the ordinate $Q Q^{\prime}$. The entropy of one pound of dry steam of the pressure and temperature shown by $Z$ is shown at $Q^{\prime \prime}$, and may be carried over into the entropyvolume quadrant by the abscissa $Q^{\prime \prime} Q^{\prime}$. The intersection of $Q Q^{\prime}$ and $Q^{\prime \prime} Q^{\prime}$ at $Q^{\prime}$ gives the latter point as a measure of the relation of entropy to volume for one pound of $d r y$ steam at the pressure shown by the point $Z$.

Referring to the Equation $2 \mathrm{I}$, page 87 , it will be remembered that when a given weight of water changes to steam by the addition of heat under constant pressure, the increments of heat, entropy, volume, and weight of vapor formed were all proportioned. Hence the relation of 
entropy to volume for a steam-and-water mixture of a given pressure must be represented by a straight line. In the case under discussion the points $R^{\prime}$ and $Q^{\prime}$ must be the extremities of such a line. Joining them gives a line to which the volume of any such mixture as $R Z$ may be referred, by means of the ordinate $Z Z^{\prime}$, to find its $c n$ tropy $O Z^{\prime \prime \prime \prime}$. Carrying across the horizontal $Z^{\prime} Z^{\prime \prime}$ until it intersects $R^{\prime \prime} Q^{\prime \prime}$, must determine the point on the entropytemperature quadrant which illustrates the thermal condition of the cylinder-feed at the point $Z$.

It will be noted that while there is only one temperaturepressure saturation-curve, $m n$, for all weights of steam and all conditions, and while there is only one pressure-volume saturation-curve, $M N$, for a given weight of steam, there are any number of entropy-volume curves similar to $R^{\prime} Q^{\prime}$ for a given weight of steam-and-water mixture, each serving for one particular pressure or temperature of mixture only.

The successive transfer of all points of the distorted indicator-card in the manner just described results in an entropy-temperature diagram of the actual cycle undergone by the cylinder-feed in the actual engine. This diagram, rotated through ninety degrees to bring it into the position in which these diagrams were always depicted in the first part of the book, would have something the appearance of Fig. 4I, although wide variations are to be expected from engines of different types and sizes.

This diagram depicts the actual thermodynamic processes of the cycle performed within the cylinder. In it may be seen just when, how, and to what extent heatenergy reaches the steam and work departs from it. It shows the advent of heat by the increase in entropy wherever the curve takes a right-hand departure. It 
shows, in literal fashion, just when this entropy falls downtemperature, doing work, the path by which it falls, and the amount of work done in the fall. It shows when and how the fallen entropy leaves the engine and by what path the unexpelled remnant is again raised to the head-level of temperature. It shows how much temperature-fall is wasted in getting the heat into the engine and how much in getting it out. It shows the leaks of heat into and out of the steam as it rises and falls, doing work, and just how much energy-waste is represented in these leaks.

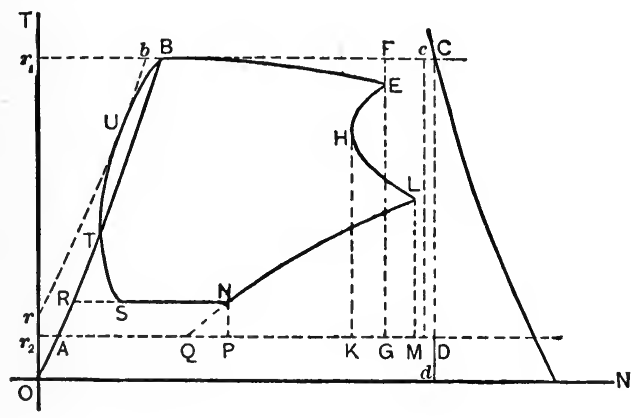

FIG. 4I

It is important to study this photograph of the cycle carefully and to separate by accurate analysis these various factors.

It is first necessary to refer this actual cycle to the one which would have been followed by a perfect engine working within the same opportunities and limitations. Since the engine is a steam-engine, the utmost that can be expected of it is the Rankine cycle. If the engine were supplied with dry saturated steam, this cycle would be shown by the lines $A B C D$; if with wet or superheated steam, this cycle would be modified as illustrated in Problems 14 and 17 . 
It is a matter of less certainty, however, as to just what should be the level of the limits at top and bottom. If the engine be a simple, non-condensing one, situated near its boiler and with ample pipe-connection between, $B C$ would clearly represent boiler-temperature and $A D$ that corresponding to the barometric pressure of the atmosphere. Should there be a long or insufficient pipe-line feeding the engine, however, it is important to distinguish between boiler-pressure and throttle-pressure. If it is desired to include and examine the evil effect of the pipe-line upon engine-efficiency, both temperature-levels may be drawn in ; and while the question of piping-losses involves many other factors than the mere thermodynamic effect upon the engine, this last would be at least clearly and accurately represented, both absolutely and as to proportion to other losses, by this graphic comparison.

If the engine be a condensing one, the problem is still more open to discussion. The simplest and, when the engine is considered alone, the most accurate plan is to draw $A D$ at the temperature-level corresponding to the actual vacuum. When the efficiency of the condenser and air-pump is to be included, however, as if this apparatus were a part of the engine whose efficiency is being analyzed, $A D$ should be drawn at the maximum, or even the mean, temperature of the condensing water.

When the engine is a multicylinder compound, the result will appear as a vertical series of such cycle-diagrams, one over the other. The gaps between represent receiverlosses. In such cases it is usually sufficient to draw between the cycles horizontal lines at the temperatures of saturation at the recorded receiver-pressures, trusting to engineering judgment to draw the proper deductions from the case without attempt at greater refinement of analysis on the graphical diagram. The question as to where the 
adiabatic $C D$ should be drawn is less simple, however, and will be taken up later as a separate topic.

Assuming that we are concerned with a single cylinder only, supplied with dry steam, the following conclusions are safe.

Wire-drawing. - When the engine took steam, it should have done so along $B C$, cut-off occurring at $C$. Instead, it does so along $B E$, cut-off occurring at $E$. The energy available for doing work at $E$ is less than that at $C$ by two quantities, viz.: (I) The triangle $B F E$, (2) the rectangle $F C D G$. The second quantity of heat lost is measured.by the rectangle between $F C$ and the axis of absolute zero of temperature, not shown on the diagram but easily drawn in, or it may be obtained as the result of a single multiplication; but only FCGD is available for doing work.

The first of these is plainly due to wire-drawing, for wiredrawing cannot rob steam of heat; it merely reduces the temperature. The loss of availability for work due to loss of temperature without loss of heat is shown by the area $B F E$, and this area is therefore chargeable against wiredrawing, as a loss due to it alone.

Initial Condensation. - The second loss, however, is due to loss of entropy, and not of temperature, and must therefore be due to an abstraction of heat. As the only known process present which can abstract heat is condensation on the cold cylinder-walls, this rectangular loss is chargeable against initial condensation.

Condensation during Expansion. - The energy available at cut-off at $E$ would indicate that a perfect engine, by following the adiabatic $E C$, might develop the cycle $A B E G A$. But instead of doing this the actual engine follows the path $E H$. Its departure to the left indicates abstraction of heat by continued condensation. Some of this heat, however, by falling from later points of the path 
$B E$ to points vertically below in $E H$, has performed some work. The loss due to this further condensation is therefore only the area between $E H$ and $K G$.

Reëvaporation. - Again, the engine, instead of expanding the steam adiabatically after condensation is complete, in which case it would follow the path $H K$, shows a deviation to the right along $H L$. This shows the addition of heat to the steam. This heat can come from no other source than the cylinder-walls, and must be due to the fact that the temperature of the steam has fallen by expansion below that of the walls, whereas originally it was above it. The addition of heat must result in the reëvaporation of some of the moisture on the cylinder-walls. The steamheat thus brought in is of course available for doing work, but by no means to the same extent as the original heat, which came in at maximum temperature. The work which it can do must be measured by the area beneath $H L$, or $H L M K$, and this area may therefore be credited as a gain due to reëvaporation.

Incomplete Expansion follows next in the list of deviations from the adiabatic path to the lower level. Instead of following the path $L M A$ back to $A$ the temperature-fall next departs to the left, when the exhaust-valve opens, along $L N$, and, if it were not arrested by a still further impediment to perfect action, would reach the $A$-level at $Q$. The triangular area $L Q M$ is therefore chargeable to exhaust before expansion is complete. It is always to be remembered, however, that for practical reasons which will be explained later complete expansion is never profitable.

This line $L Q$, when compared with a theoretic constantvolume curve through $L$, gives the exaggeration of the loss due to incomplete expansion by clearance. This consideration, with those discussed on pages 223-225, gives an absolute measure of the deleterious effect of clearance. 
Back-pressure. - It next develops that the cycle never attains the lowest possible temperature-limit, $D A$. It never falls below $N R$. The area representing the resultant loss, $N Q A R$, usually measures a nominal rather than a real loss. Some back-pressure is inevitable. To supply ports and valves of the enormous size necessary for eliminating it would be very foolish. As with the loss due to incomplete expansion, the only question is whether it be excessive or not.

Cushion and Clearance. - With the next deviation from the theoretic, that of the path $S T U B$ from the water-curve $R B$, arises a much more pregnant question of steam-engine design. This question lies between two broad types of steam-engine: (I) Those designed to have a minimum possible clearance, with little or no cushion, and (2) those designed to have a liberal clearance, which is to be filled with cushion-steam at each stroke compressed to as nearly boilerpressure as is practicable. The first is naturally the dropcut-off, slow-speed type; the second is the positive-gear, high-speed type. Before discussing the question, however, for it is a lengthy one, it were well to decide just how the amount of heat-work lost during compression is to be measured from the actual cycle before us.

It is obvious that at the point $S$ the cylinder-feed possesses more heat than it theoretically should, and that during compression heat to the amount STR is abstracted. This little triangular area reveals the departure from the cylinder of exhaust-heat along the line $S T$ which should have gone out by the path $S R$.

At some point in the compression, however, as at $U$, the cycle shows the cylinder-feed as having less heat than it would if it were water of the given temperature, which is manifestly impossible. What has happened is plainly that the cushion-steam, which alone occupies the cylinder in the actual engine, although theoretically all of the cylinder-feed 
is present in the form of water, has lost some heat to the walls during its compression. The mathematical elimination of the cushion-steam, however, causes this loss to appear as one drawn from the cylinder-feed.

At first sight it might appear that what was wanted was an analysis of what happened to the cushion-steam during its compression. In reality, however, such determinations are misleading. For instance, it might thus be found that the cushion-steam at some point in its compression consisted of $50 \%$ water. This would seem a tremendous loss of heat, which should be remedied at any cost. If, however, the cushion-steam amounted to only 10\% of the cylinder-feed, the lost heat reduces to $5 \%$ in terms of cylinder-feed, or one of the smallest.in the engine. And since it is cylinder-feed alone which is a source of expense to the engine-user, all losses must be reduced to that basis of comparison.

It is obvious, too, that all heat lost by the cushion-steam during compression must be made good by the cylinderfeed entering at the next stroke before the latter can begin to accomplish any useful result. It is therefore quite proper to consider the point where the heat-abstraction from the cushion-steam reaches the extreme as the zero of all heat-gains and losses by the cylinder-feed. This is easily done graphically by drawing through the point $U$ a curve parallel to $B R$, such as $b U r$, tangent to $B T R$. This arbitrarily moves all reference-points to the left by the distance $B b$. A new limiting adiabatic must therefore be drawn parallel to $C D$ and at the same distance to the left, as at $c M$. This alters the apparent value of heat lost in initial condensation from that prescribed on page $22 \mathrm{I}$. The other losses and gains are unaltered. What is in reality true is that a large portion of the initial condensation occurring in every cylinder is due to heat-losses 
incurred before any steam was admitted, which losses are stored up by the cushion-steam for draft upon the cylinderfeed when the latter shall next appear in the cylinder. The process just defined separates these two responsibilities for condensation. The area $U r S U B b U$ is chargeable against clearance and cushion-steam. The area $F c M G$ is due to condensation which occurs after the clearance has been filled with boiler-steam and which would take place were the clearance zero. It is this preliminary demand for boiler-steam to reimburse the cushion-steam and to carry on the preliminary condensation which occurs before the stroke begins which constitutes the real reason for the lead of the valve. When it is remembered that the greatest part of the surface exposed to boiler-steam by the time cutoff takes place is already exposed to it when admission takes place into the clearance-space at dead-centre, it is obvious that there is good basis for expecting this preliminary demand for steam to constitute a goodly proportion of the total supply.

It is also obvious, from the foregoing argument, that it is the clearance-surface, rather than the clearance-volume, which is so deleterious to steam-engine efficiency.

As the assumption of initial dryness on the part of the cushion-steam is the only assumption entering the entire analysis, it may be insisted that the conclusions reached by means of the entropy method are exact to an unusual degree. If, therefore, the entropy-analysis should reveal that the area chargeable to cushion-condensation was so great, in any given instance, as to overbalance any gain from diminished first cost, space, etc., which the cushion, through the medium of the high rotative speed which it permits, has developed, then the minimum-clearance design should obviously be chosen in preference for this particular service. 
With the foregoing means of obtaining an exact knowledge of existing facts as a basis, some very plain and simple conclusions may be drawn concerning several features of steam-engine design which frequently find themselves under discussion.

\section{Size ANd Speed}

If by the size of an engine is meant the dimensions of its cylinder, then it may be stated as a well-known fact that the larger the cylinder, the less is the condensation, because the less the surface exposed per pound of cylinder-feed. The internal surface of similarly shaped vessels, such as engine-cylinders, must plainly increase directly with the square of any one dimension. But as the cubical contents at the same time increase directly with the cube of the same dimensions, it is plain that the ratio between cylindersurface and the amount of steam contained must vary inversely as the first power of the same dimension; whence, the larger the cylinder, the less will be the percentage of condensation.

More commonly and more accurately, however, the size of an engine is understood to refer to its power. This being so, is it equally true to say that the larger the engine, the less the condensation? If the variation in power between two engines subject to comparison is gotten by varying the cylinder-dimensions, while speed and pressure are kept constant, then it is plain that the proposition still holds true. If, however, the increased power be gotten by increased speed, the cylinder-dimensions being kept constant, the problem is altered. Here the same surface is exposed to the same fluctuations of temperature a greater number of times per minute. As the amount of cylinderfeed is also roughly proportioned to the speed, the proportion of condensation could only remain the same, as the 
speed increased, if the absolute quantity during each cycle of temperature remained constant. But as it takes time to transfer heat from steam to cylinder-wall and back again, and as the time occupied by each cycle diminishes as the speed increases, the absolute condensation per cycle will plainly not remain the same; instead, it will decrease, and hence the proportion of condensation suffered by the cylinder-feed must be said to decrease as the speed increases. But, whereas it was quite safe to assume that in alterations of cylinder-dimension the amount of condensation varied in true and simple proportion to the surface, it is not by any means probable that the condensation upon any given surface is proportional to the time of exposure. For the heat is not carried away by the metal, but is merely stored up within itself, and it is easy to imagine that the first heat imparted to the walls might raise its temperature sufficiently toward that of the steam so that the rate of heat-transmission would be substantially altered. Indeed, it is safe to say that the condensation must increase with the time of exposure in some ratio which is some fractional power of the time. If this should be true, then it would appear that it were more profitable, so far as condensation is concerned, to increase an engine's power by increasing the size of cylinder rather than by speeding up, and as purely mechanical argument also advises the running of larger cylinders at slower speeds, it is plain how thermodynamics and mechanics coincide in supporting the natural tendency visible in actual designs.

But it is also to be noted that since increase in speed is actually conducive to thermodynamic efficiency, but only less so than increase in dimension, the running of largepowered engines at high speed finds no censure in thermodynamic argument, when it is warranted by mechanical considerations. 


\section{STEAM-PRESSURE}

As the range of pressure, and therefore of temperature, in an engine increases, the theoretic efficiency plainly increases. At the same time, by reason of this same temperature-range, the condensation must increase. At first, as one starts from a very low pressure-range, the theoretic gain will be very great because it is almost fully proportional to the temperature-range and because the latter increases, in the lower field of pressures, more rapidly than the pressure. But as higher pressures are reached, all of these conditions are first annulled and then reversed, and the increase in condensation is found to more than offset the gain in heat-available-for-work. The point where balance is established between the two gives the maximum pressure-range which it is profitable to impose upon the cylinder for purely thermodynamic considerations; though for other reasons higher pressures are often found profitable in special services, such as locomotives or torpedoboats, where it is all-important to get a maximum of power out of each inch or pound of engine.

This limit may be determined only by experiment upon each type of engine considered. For reasons stated in the preceding article, it is plain that the limit would be lower in small engines than in large. It is generally understood to lie between $90^{\circ}$ and $120^{\circ} \mathrm{F}$. of temperature-range within a single cylinder, the lower figure for small cylinders and the higher for large ones. It should also be understood, however that when applied to unjacketed cylinders these figures give rather the maximum range obtainable without real detriment from condensation than the minimum range permissible without net loss from thermodynamic reasons; for the natural tendency is always to take the greatest permissible range, for the sake of capacity or power, rather than the least. 


\section{The Steam-Jacket}

It is the rôle of the steam-jacket to prevent or minimize condensation in the cylinder. Since the heat supplied to the steam through the medium of the steam-jacket is good boiler-heat, obtained at as great an expense as steam-heat entering by way of the throttle, some explanation is necessary as to why any gain in efficiency is possible by its aid. Indeed, since the steam-jacket is free to radiate heat into the exhaust during half the time, and since, even during the working-stroke, a large part of the heat supplied by it to the working-steam is imparted after cut-off, when the availability for doing work is much less than is the case with boiler-steam, explanation is also necessary why the steam-jacket should not constitute a distinct source of loss of efficiency. The answer to both points is found in the fact that the one thing in an engine-cylinder which is able to very much exaggerate the detrimental influence of the cylinder-walls is moisture. Able to exchange heat with the steam much more rapidly than can the iron of the walls, and having, too, a much higher specific heat, water is very much more active than metal in carrying on that condensation and reëvaporation which has always been the chief bar to the attainment of maximum theoretical efficiency.

It is not a sufficient guard against the phenomenon to provide the engine with perfectly dry steam, although the presence of wet steam makes the situation very much worse than if it were dry; for even with dry steam the initial condensation which must take place upon the walls as the clearance fills with boiler-steam forms a nucleus of moisture which invites additions in a geometrical ratio.

The steam-jacket prevents the appreciable accumulation of moisture upon the internal surface of the cylinder-walls 
by warming up the cylinder with steam-heat, the resultant moisture from which remains outside the cylinder and is drained away. That is the real and essential difference between a jacketed and an unjacketed cylinder. The heat supplied from the boiler for radiation is just as great and just as costly in either case; but in the former the resultant moisture does not remain in the cylinder as an active carrier of heat into the exhaust, whereas in the latter it does.

There is an additional gain, too, due to the fact that the mean temperature of the cylinder-walls is kept nearer to boiler-temperature. Because the walls are open to the exhaust during the back-stroke, the steam-jacket cannot quite bring them to boiler-temperature. But the initial condensation is much decreased in amount, and its reëvaporation, instead of awaiting the fall of temperature within the body of expanding steam below a mean between boiler and exhaust-temperatures for its inception, begins as soon as cut-off takes place and usually finishes before the workingstroke is complete. This being so, the steam-jacket can lose very little heat to the comparatively dry exhaust-steam, and by the time cushion takes place can get the walls well back toward boiler-temperature. The result of its action therefore is :-

(I) To much decrease the amount of boiler-heat undergoing free fall within the cylinder by keeping out of the cylinder the moisture developed in heating it ;

(2) To impart the bulk of what does undergo this free fall of temperature to the working-steam at an earlier point in the cycle, before the latter's expansion is complete, whereby a portion of its temperature-fall to exhaust-temperature is utilized.

Since the prime office of the steam-jacket is to diminish condensation, it must be of the most value where conden- 
sation is excessive; that is, where a high thermodynamic efficiency is, or is thought to be, valuable, and is sought through the medium of (I) a large ratio of expansion or (2) slow speed of rotation. Under these conditions a good jacket will show an increase of efficiency varying from 5 to $15 \%$, with probably $7 \%$ or $8 \%$ as a fair average.

It is to be especially noted, however, that under wrong conditions the steam-jacket may become a source of expense instead of economy. The majority of engine-builders, rating small and large together, omit it from their designs, and in this the majority of them are right. In debating the question as to when jackets are desirable and when they are not, the first fact to be noted is that a steamjacket is of itself a very expensive and undesirable feature in an engine, and the saving which it develops must be a substantial and a certain one if the net result of its addition to the engine is not to be a distinct loss. A jacket is difficult and expensive to construct. The core for it is large and thin. Its presence usually entails coring several other portions of the casting where cores would otherwise be unnecessary. The percentage of cylinder-castings lost in the foundry is very much higher with jackets than without. The joints between barrel and head of cylinder at either end are much complicated by its presence. If it is remembered that the great bulk of the condensation of the working-steam takes place in the clearance while the piston is at, or very near to, the end of its stroke, it will be plain that the portions of the engine which especially need to be jacketed are the cylinder-heads, and to connect these jackets with those around the barrel and to avoid too close contact with the stuffing-box, all involves difficulty and expense in construction and maintenance. Therefore, while it is undoubtedly true that a steam-jacket would somewhat increase the thermodynamic efficiency of any cylinder whatever, it 
is only when this increase is substantial that it may counterbalance the commercial losses involved in its adoption.

The settlement of this question necessarily involves an understanding of the relation existing between thermodynamic and commercial arguments in any given case. But its discussion applies with equal weight to all of the factors which enter into engine-efficiency which have already been discussed: size, speed, ratio of expansion, clearance, and cushion. It is therefore proper to reconsider them all in the light of this one argument.

The design of a steam-engine, as is true of any merchantable article, properly follows the lines upon which demand is expressed. The demand coming from the buyers of steam-engines naturally divides itself into two broad sorts, if minor differences due to special conditions be ignored. Of these, the first comes from him to whom the item of power is a large and important one in his business; the second comes from him to whom power is a minor factor. Examples of the first are electric lighting, electric generation in general, large textile mills, paper mills, marine propulsion, etc. Examples of the second are machine-shops and the general run of factories of moderate size wherein, as in the machine-shop, the pay-roll or the cost of raw material is a very much larger item than is the cost of power. All of the former class have presumably plenty of capital to invest in steam-plant, for that is their business, and return upon that capital will be a maximum when the coal-consumption is the least. Of the second class, those who have plenty of capital invest it in acquiring the best plant possible of the sort which really does the earning of the returns from their business, and which consists of machine-tools, overhead cranes, and similar facilities for doing the best possible work in the minimum of time and 
with the least waste of raw material or labor. The power plant of such a factory is properly a secondary consideration. To it, therefore, is accorded only sufficient capital to prevent its being actually bad.

In addition to this latter class, too, should be considered those to whom the cost of power is a prominent item in the cost of doing work, but who have to approach every department of their business, owing to insufficient capital, in the attitude of cutting down investment to the lowest possible point without regard, always, to whether further investment might not be profitable.

These two sorts of buyers therefore demand, the one an engine which will operate upon the highest fuel-efficiency without regard to first cost, the other an engine which will bring the first cost for power and its incidentals to the lowest possible figure without regard to more than a moderate degree of efficiency. It so happens that these two attitudes lead to decisions in regard to the several points of design just discussed which are singularly harmonious in their combination into a complete type of engine. These points are :-

I. Degree of expansion, including the question of compounding.

2. Valve-motion.

5. Speed.

3. Clearance.

4. Cushion.

6. Steam-jacketing.

7. Condensation.

I. Degree of expansion. - It is plain that the greater the ratio of expansion, the greater is the ideal efficiency. It is also obvious that the greater the degree of expansion, the greater are, not only the thermodynamic losses due to cylinder-condensation, etc., but also all those expenses due to larger cylinders and earlier cut-offs as well; for the entire cost of an engine-plant is closely proportional to the size of 
cylinder. A comparatively late cut-off may be easily gotten by means of a single valve and a very simple valve-motion; but to attain an early cut-off, and at the same time preserve the proper distribution of exhaust and compression, demands the use of from two to four separate valves, and often more than one eccentric. Therefore it can be safely said that there is one broad type of engine which carries the attainment of efficiency by means of higher ratios of expansion at the expense of larger cylinders and more complicated valve-gear; there is another which foregoes this efficiency to an appreciable degree, to the end that cylinders may be small and the valye-gear simple. In this connection it ought also to be remembered that large cylinders mean larger engines in every respect, and larger engines mean heavier and more costly foundations, more care, more oil, more space about them, heavier cranes overhead, etc., etc. In fact, it may be safely said that the entire cost of construction of engine-room and its maintenance, aside from the cost of fuel, bears a rough but fairly accurate proportion to the size of cylinder employed.

The foregoing remarks apply equally to the question of the degree of completeness of expansion, or of terminal pressure, which is somewhat independent of the question of ratio of expansion. It is a very common fallacy that the indicator-card with the sharpest toe, other things being equal, shows the best efficiency. So false is this that it may be broadly and responsibly stated that any indicatorcard with a sharp toe is inherently bad. The justification of this statement lies in the following argument: It is obvious that of the pressure in the cylinder at any moment only that exerting a pull upon the belt is of any value to the engine-owner; the rest is expended in merely keeping the engine in motion. In other words, if we translate engine-friction into its equivalent in mean pressure upon 
the piston, we shall find the equivalent to amount to from about 4 pounds per square inch for large low-pressure cylinders to about 15 pounds for high-pressure cylinders. Whenever the pressure upon the piston is less than this, the belt must be dragging the piston after it, and the net result is a distinct loss. Moreover, since every inch of cylinder costs money to build, to erect, to maintain, and to operate it, the engine is a distinct source of loss until it is developing enough power of sufficient net value to make good this loss. If this power be also translated into mean effective pressure and added to the former figure, the results will vary between the extreme limits of about 5 and 25 pounds, or ordinarily between 7 and 20 pounds, per square inch of mean effective pressure. Of these figures the smaller apply to low-pressure, the larger to high-pressure cylinders. If the terminal pressure in the cylinder be allowed to fall below these figures, it is proof that the engine is not developing and delivering to the belt enough moneymaking power to fully pay for its keep. The plant would be more efficient under a later cut-off, either in a smaller cylinder or in the same cylinder under lower initial pressure.

The wide prevalence of the fallacy referred to in this connection is almost wholly due to the absurd practice of uniformly making guarantees and reports of engine-efficiencies upon the basis of the indicated instead of upon the brake horse-power. Deception as to what is a profitable degree of expansion is only one of the many ways in which the engineering public loses from this habit.

The question of the profit of compounding or not comes under exactly the same argument. The details of the science of compounding will be treated later under the proper heading; but it is important to note that the commercial efficiency of a steam-plant is not necessarily improved by 
compounding, and that many existing compound engines are operated at a loss compared with what might be accomplished by a single cylinder. But in the great majority of cases the mistake is in the other direction. Single cylinders are adhered to for the sake of cheapness when the additional money needed for compounding would earn a far greater return than any other money invested in the business.

It might also be misapprehended from the above that compounding should only be regarded as an exaggeration of the policy of the short cut-off, four-valve type of engine, and to be adopted only after passing through this preliminary degree of search after efficiency. This is not true, however. There are many mechanical and commercial advantages to be conserved by retaining the compact, single-valve, high-speed type of machine, even when search ing after greater efficiency by means of compounding. But the resultant type is necessarily an intermediate and more or less special one, limited properly to a comparatively narrow, although absolutely a very large, field of sizes and of diversity of employment.

2. Valve-motion. - This point has already been partly covered. The valves and gear fit to produce early cut-offs are comparatively expensive to build and to maintain. The question is also intimately connected with that of

3. Clearance. - It happens that the valves fit for producing a very short and sharp cut-off also permit the attainment of a very small clearance in the cylinder. As clearance has been shown to be always somewhat detrimental to efficiency, here again the coincidence is harmonious with the other points in dictating large cylinders, four valves, and small clearance as characteristic of the more efficient type. It also happens that the best forms of single-valve engines always involve large cylinder-clearance. This brings into the discussion the next point:- 
4. Cushion. - The detrimental effects of clearance can be very largely, although not entirely, counteracted by the use of sufficient cushion to bring the pressure in the clearance approximately to boiler-pressure by the time deadcentre is reached. It also happens that when a single valve and eccentric are used, the attainment of a cut-off earlier than about three-quarters stroke is impossible without making the exhaust-closure also correspondingly early. So that it appears that in the type of engine which needs cushion to make it efficient considerable cushion is mechanically inevitable. It is a fortunate coincidence that in this type of engine a great deal of cushion may be had very easily.

5. Speed. - It was pointed out in the analysis of the causes of cylinder-condensation that, for an engine of any given power, that phenomenon was probably not at all affected by speed. But the many items of cost of operation of an engine due to causes other than cylinder-condensation are all very sharply affected by speed. The higher the speed, the smaller and cheaper are the cylinder, the foundations, and the engine-room, the lighter are the belts and shafting, and the better the regulation. That there are any mechanical disadvantages or costs to be incurred, under proper design, from high engine-speed is not to be urged to-day. Twenty years ago high speed had to apologize and fight for itself, as a mechanical monstrosity. Today even the advocates of the slowest speeds have so increased their rates of rotation that it is generally recognized that the arguments in favor of slow engines are based upon other points than purely mechanical ones. But one of the essential points in good design for high speed is plenty of cushion. It is therefore another one in the string of happy coincidences that the type of engine which naturally adopts the highest possible speed and the simplest 
possible valve-gear is forced by the latter and permitted by its clearance to have just what is demanded by its speed, viz. : a high degree of cushion. On the other hand, the type of engine which naturally adopts a comparatively slow speed and a large cylinder is permitted, by the complex valves and gear which it needs and can pay for, to have very little clearance; consequently it may have little or no cushion. But cushion it does not need.

6. Steam-jacketing. - From the characteristic aims which have already been mentioned as outlining these two types of engine it is plain that, were the steam-jacket to develop under all conditions of operation the same average gain of efficiency, it would be quite likely that the large, slow-speed engine would find it profitable to adopt it, whereas the single-valve type would not. But if the conditions which exaggerate or minimize the effectiveness of jacketing be contrasted for the two types, it is seen that the four-valve engine possesses all of the former and the single-valve all of the latter. Large cylinders, slow speed, and short cutoff all increase the need and the effectiveness of a jacket; and the type which embodies these features can afford to pay for a jacket if it will even perceptibly aid efficiency. Small, compact cylinders, high speed, and moderate cut-off lead to moderate condensation, so that a jacket would have little chance of doing good; at the same time, the engine embodying these features cannot afford the complication of a jacket, nor does it care to go after increased cylinderefficiency even were it more marked than under these conditions would be the case.

7. Condensation or Non-condensation of Exhaust. - This point in design is different from the others in that its feasibility depends very largely upon a purely external and largely fortuitous circumstance, viz.: the existence of a proper supply of cheap condensing water. The amount 
of water needed is from one to two gallons per horse-power per minute. The amount of coal to be saved by its use is, to give a single figure which will roughly average all the varying conditions, not far from one pound per horsepower per hour. But even if it be true, in any particular case, that the water-supply be present and that the cost of condensation has been rightly interpreted, it is yet to be remembered, in close analogy to the preceding argument, that the conditions which speak for the adoption of condensation also dictate the use of the four-valve type of engine. On the other hand, conditions which would lead naturally to the choice of a single-cylinder type of engine would hardly warrant the adoption of the expensive complexities incidental to working under a vacuum. The crossing of these natural combinations - that is, of a single-valve engine with a condenser, or of a four-valve engine with atmospheric exhaust where condensation is possible - may be accepted upon its face as irrational and wasteful.

To summarize: There is no thermodynamic reason why engines of the highest speeds should not have the highest efficiencies. The work of Willans and Rites has proven this. The reasons why they usually do not are economic or commercial in character.

On the other hand, there are reasons both thermodynamic and economic in character why the high-speed type of engine excels the other in sizes below 75 to 100 horse-power. In the writer's opinion four-valve engines smaller than this should become as thoroughly obsolete as should the throttlegoverned engine of any size whatever. 


\section{CHAPTER II}

\section{THE COMPOUND STEAM-ENGINE}

THE study of the compound engine must be approached from the standpoints of two very distinct sets of considerations: (I) the mechanical, and (2) the thermal. In the development of the steam-engine, compounding was attempted by Woolf at a very early date, late in the eighteenth century, in fact, but was unsuccessful owing to tardiness of development of purely external considerations. More than half a century elapsed before McNaught introduced it again, and then it appeared chiefly as a modification of existing engines to permit the use of higher steam-pressure and the development of greater power. The objects sought were purely mechanical. It was quite as a secondary and incidental result that it appeared that the efficiency also was increased.

But McNaught's method of compounding applied only to the heavy stationary beam-engines of the first half of the last century, and found a very limited adoption. By I860, however, it had come to be a pretty well established fact that the larger marine engines must include more than one working-cylinder and crank, for purely mechanical reasons. Weights of individual parts were thereby reduced, evenness of turning-moment on the shaft was much enhanced, the higher rotative speeds demanded by the substitution of the screw for the paddle-wheel were facilitated, and the danger of complete failure of power from the failure of any one small piece in the engine was considerably decreased. Then, the gain in efficiency from 
compounding having been recognized from McNaught's experience, the substitution of two cylinders of dissimilar dimensions using the steam in series for two cylinders of equal size using it in parallel was an exceedingly simple, easy, and inexpensive step. Nevertheless it was one of supreme importance for the development of the world's maritime trade and of the steam-engine in all services. Upon the increased efficiency due to the adoption of compounding in marine engines was based the tremendous expansion of the merchant marine in radius of steaming ability, in speed, and in cargo-carrying capacity. In the earlier days of simple engines the transatlantic voyage was apt to consume twenty days, and the daily consumption of coal was such that a vessel could scarcely carry her own fuel for a longer voyage; certainly not with a proper margin of freight-paying cargo. But as compounding made the engines more efficient more power could be put on board, shortening the time-length of a given voyage; the decreased time joined hands with the increased efficiency to reduce the demand upon cargo-space for fuel.

By $1875-80$ the growth of the ocean liner had attained such a size that propelling engines upwards of ten to fifteen thousand horse-power had become common. All of these engines were compounded, of course; but the same considerations which had led to the splitting of the non-compound engine of 1850 into two cylinders now forced the adoption of three or more cylinders working upon three separate cranks in the design of these big compound engines. The high-pressure engine was usually placed in the centre, with two low-pressure cylinders forward and aft of it respectively. The cranks were usually set at an angle of $120^{\circ}$ apart; the steam-pressure was ordinarily between 100 and 125 pounds.

When the triple compound was introduced (I880-85) 
as a more efficient and no more expensive way of building the standard three-cylinder engine, it soon led to engines of still larger power, because under the improved efficiency the limited coal-bunkers and boiler-weights would permit it; and as the size of engine increased, four or more cylinders and cranks were adopted in triple-compounding just as three had been used in double-compounding.

To-day it is an accepted fact that the marine engine of any appreciable size must have at least four separate cylinders, and often more, working three to five separate cranks. The steam-distribution in these cylinders may be upon the double, triple, or quadruple plan of compounding, according to the special conditions of the service in view, and to the designer's estimate of the thermodynamic gain to be expected; but the multiplication of cylinders has been dictated by purely mechanical considerations and has lain back of and quite independent of all thermodynamic factors. For this reason the mechanical features of the question of compound $v s$. simple engines will be taken up first, although in mere outline, and the thermodynamic considerations left to a secondary consideration.

Mechanical Differences. - To gain a clear idea of what is meant by compounding reference must be had to Fig. 42 .

Let $B B^{\prime}$ represent the given boiler-pressure, $A A^{\prime}$ the atmospheric line, and $C C^{\prime}$ the vacuum in the condenser. Then, with a given supply of boiler-steam $B D$, the power available is given by the area $B D C^{\prime} C$, supposing the curve $D R C^{\prime \prime}$ to be extended to intersect $C C^{\prime}$. This power would ordinarily be developed in a cylinder having the total volume $C C^{\prime}$ and cutting off at $\frac{B D}{C C^{\prime}}$ of the stroke. If it be desired to use compounding, however, a high-pressure cylinder may be added to the engine and an intermediate receiver introduced between the two cylinders. 
Let the pressure in the receiver be $R R$. This pressure is determined by the volume at cut-off in the low-pressure cylinder $R R$, which is, of course, capable of adjustment. The indicator-card of the high-pressure cylinder now becomes $B D R R$, and that of the low-pressure cylinder $R R C^{\prime} C$. If it is desired to limit the effective terminal pressure to the profitable minimum defined on page 234, making both cylinders smaller will cut off the toes of the

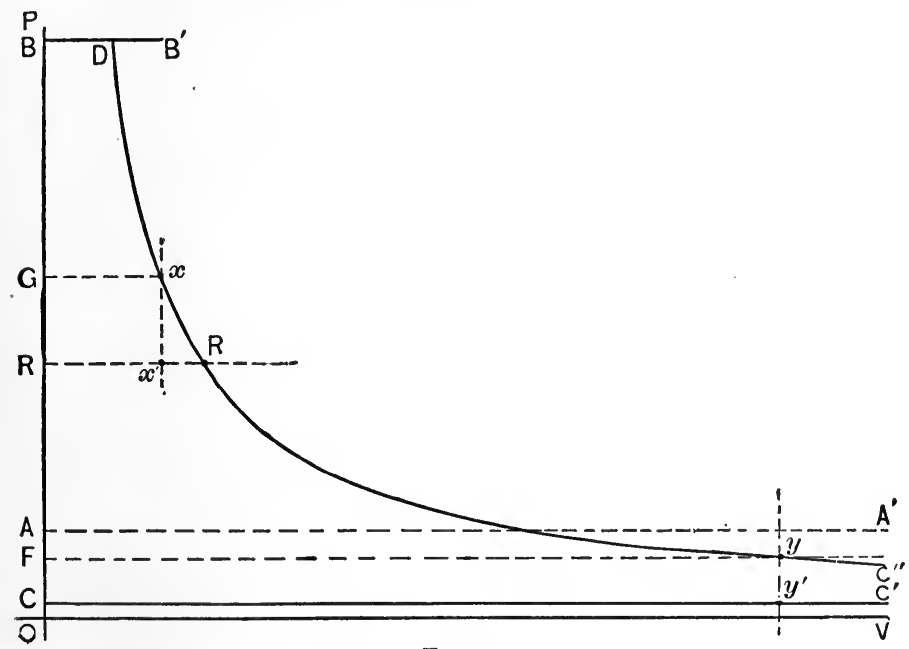

FIG. 42

cards. If the volume of the high-pressure cylinder be reduced from $R R$ to $G x$, and that of the low-pressure cylinder be reduced from $C C$ to $F y$, the toes of the cards will be cut off at $x x^{\prime}$ and $y y^{\prime}$ respectively.

In triple compounding there would be two such dividing lines as $R R$, representing the pressures in the first and second receivers respectively; in quadruple compounding there would be three such horizontal lines.

The first and most obvious conclusion from this is that the total area of available work is no greater after com- 
pounding than it was before. Indeed, it is somewhat smaller by the loss of pressure inevitable in passing the steam from one cylinder to another. From this broad fact is deduced the rule that -

In a given compound engine, under a given boiler-pressure and a given ratio of expansion, the power developed is independent of the number of cylinders through which the steam is worked before reaching the low-pressure cylinder; it is the same as if the same boiler-steam performed the same number of expansions in the low-pressure cylinder alone.

The simplest form of compound engine is the tandem, where both pistons work upon the same piston-rod and crank. With the exception of the compound beam-engine of McNaught, which never found a wide acceptance, although some very large engines have been built on his plan, it was also the first. It is undoubtedly the cheapest to build, and so finds wide acceptance yet, for stationary engines of moderate power. Its latest field of adoption is in locomotive work. Here it contests for the laurels with the Vauclain arrangement of a pair of parallel cylinders, high-pressure and low-pressure, on each side of the locomotive, each pair working on a single cross-head or with the several European types of four-cylinder compounds. The two-cylinder compound locomotive, with a high-pressure cylinder upon one side and a low-pressure cylinder on the other, does not promise to be a permanent type.

The purely mechanical advantages of compounding have been stated as consisting of lighter weights all around, a more even turning-moment, better equalized strains upon all working-parts, and greater ease in properly distributing the steam. It would seem, at first sight, that in the tandem compound none of these advantages could appear; for since a single crank and set of connections is relied upon 
to develop the same power in either case, the difference would appear to lie wholly within the cylinders, and that their complexity and weight must be doubled. Upon investigation, however, such does not appear to be the case.

Referring to Fig. 42, let a simple-cylinder engine be compared with a tandem-compound, supposing that in each case the boiler-pressure be 145 pounds by gage, or I60 pounds absolute, and that the final volume $F y$, in order to develop the desired power, must be ro cubic feet. This may be considered as supplied by a cylinder $27^{\prime \prime} \times 30^{\prime \prime}$, which would constitute the only cylinder of the simple engine and the low-pressure cylinder of the compound. To the latter must be added the high-pressure cylinder upon the same axis with the low-pressure cylinder, with their pistons attached to a common piston-rod. If the proper ratio between the two be assumed to be four to one, this would call for a high-pressure cylinder $13 \frac{1}{2}{ }^{\prime \prime} \times 30^{\prime \prime}$.

In the case of the simple engine the maximum pressure upon the piston, which would occur just at the beginning of the stroke, would be (neglecting back-pressure) I60 pounds over an area of 572 square inches, or a total of 91,520 pounds. The cylinder, piston, piston-rod, connecting-rod, crank, shaft, and engine-bed must all be designed to withstand this stress.

In the case of the compound (if the simple empirical equation of $p v=$ a constant be assumed as a basis for estimates) the receiver-pressure should be $\frac{1}{4}$ of $160=40$ pounds absolute. Then the high-pressure piston would be subjected to a maximum stress of $\mathrm{I} 20$ pounds over an area of 143 square inches, or a total of $\mathrm{I} 7, \mathrm{I} 60$ pounds. The low-pressure piston would be subject to a maximum pressure of 40 pounds over an area of 572 square inches, or a total of 22,880 pounds.

It is immediately obvious that, in so far as cylinders and 
pistons alone are concerned, they need to be built to withstand a stress only about one-quarter as large as before. Not only will they be much cheaper to build and handle, but all their appurtenances in the way of valves and gear will be much lighter, cheaper, and more reliable, and enginefriction will be much reduced. Even when attention is turned to cross-head, crank, and connections a great gain is visible. The total maximum stress upon them in the case of the compound is 40,040 pounds as compared with 91,520 pounds for the simple engine, representing a gain of over $60 \%$.

The explanation of the great difference, for the same power developed on a single crank, lies in the fact that whereas in the simple engine the maximum stress would continue only throughout about one-sixteenth of the stroke (if a terminal pressure of ro pounds be assumed), in the compound the cut-off would not take place until one-quarter of the stroke had elapsed. The pressure would be more moderate in the case of the compound, but would be more continuously sustained.

If attention be turned from the tandem-compound to the cross-compound, where each cylinder has its own crank, or to the marine-type of compound, where the work developed in a single one of the stages is often distributed between two cylinders and cranks, the degree to which this gain in lightness and strength may be exaggerated can be well imagined. In the last-named type, too, an additional argument for multiplication of stages of compounding, as well as of cylinders and cranks, lies in the fact that these engines can carry no fly-wheel and possess light foundations, and therefore require a very even turning-moment on the shaft. A short cut-off under high pressure upon a single large piston would start off the stroke with a disastrously violent jump. At the other end of the stroke, too, since the re- 
versing valve-gear under which all marine engines must work introduces a heavy cushion as an inevitable accompaniment to an early cut-off, the piston would be arrested in its motion by an excessive negative pressure. The engine would either fail to pass dead-centre altogether, or would do so reluctantly and with noise or vibration. Compounding remedies all of these troubles. As even so moderate a cut-off as one-quarter stroke, however, would develop a troublesome cushion, the designer of a marine engine to work under a pressure as high as was assumed in the above illustration would choose between the alternatives of a less complete expansion on the one hand or of the extra cost and complexity of triple-compounding on the other. Thus, in the illustration, a terminal pressure of ro pounds absolute was assumed in the low-pressure cylinder and complete expansion to receiver-pressure in the high-pressure cylinder. This would make the indicator-card of the former follow the diagram $R R y y^{\prime} C$, Fig. 42, and the latter the diagram $B D R R$. If, however, the stroke of each piston were shortened from 30 to 24 inches, the terminal pressure in the high-pressure cylinder would be raised to

$$
\frac{B D}{G x} \cdot B O=\frac{7 \cdot 5}{24} \times 160=50 \text { pounds, }
$$

and in the low-pressure cylinder to

$$
\frac{R R}{F y} \cdot R O=\frac{7.5}{24} \times 40=12.5 \text { pounds }
$$

absolute, or Io pounds net; which is much better engineering practice. The cut-off would have been lengthened from 25 to $3 \mathrm{I} \%$ of the stroke and the excessive cushion have been moderated thereby.

Let it be supposed again, to take up the second alternative in comparison, that this same expansion were to be 
carried out in a four-crank, four-cylinder, triple-compound engine, having one high-pressure, one intermediate, and two low-pressure cylinders. Since the last stage of the expansion is to be divided between two cranks, a little extra proportion of expansion and of power may be thrown into the low-pressure stage. This could be done, for instance, by giving the engine two low-pressure cylinders of 19 inches in diameter, one intermediate cylinder 15 inches in diameter, and one high-pressure cylinder 10 inches in diameter; the stroke of all of them may be considered as still 30 inches. It is not to be considered by the student that this necessarily represents the best engineering practice, but it enables a simple comparison to be drawn between engines of widely different type under the same steam-pressure and expansion, in order to bring out most plainly one or two particular points; viz., comparative maximum stresses and comparative cut-offs. The dimensions chosen give a ratio between high-pressure and intermediate of 2.3 I, between intermediate and low-pressure the same, and 3 as the ratio of expansion within the low-pressure cylinder. This places the first-receiver pressure at $160 \div 2.3 \mathrm{I}=69.3$ pounds, and the second at $69.3 \div 2.3 \mathrm{I}=30$ pounds absolute. The maximum pressure falling upon the high-pressure piston and crank is $(160-69.3) \times 78.5=7120$ pounds. That upon the intermediate piston and crank is $(69.3-30) \times 177=6956$ pounds. That upon each low-pressure piston and crank is $30 \times 283$ $=8490$ pounds. If these figures be compared with the maximum stress on a single crank of 17,280 pounds for the two-crank cross-compound, 40,320 pounds for the tandemcompound, and 92, 160 pounds for the simple engine, the gain is obvious. The cut-off in each of the two smaller cylinders of the four-cylinder triple-compound would be $43.3 \%$ and in the largest cylinders $33.3 \%$.

Finally, were the strokes again reduced to 24 inches in 
order to give a profitable terminal pressure and to minimize cushion, the cut-offs would lengthen from $43 \%$ to $54 \%$ and from $33 \%$ to $42 \%$ respectively.

Although intended for illustration only, these proportions, if modified so as to give a shorter stroke and larger diameter of cylinder and worked under a higher steampressure than 145 pounds by gage, would represent a close approach to good marine-engine practice.

\section{Thermal Considerations}

It will be remembered that in the discussion of cylindercondensation and its kindred phenomena it was noted that both theoretic efficiency and condensation increase as the temperature-range within the cylinder increases. The former increases almost proportionately to the temperature-range; the latter, insignificant at first and increasing very slowly with the first growth of temperature-range, takes on an ever-increasing augmentation as the additions to the temperature-range accumulate, in somewhat geometric ratio, until finally cylinder-condensation becomes the all-important phenomenon, overbalancing and eclipsing all other considerations. In other words, as the theoretic efficiency increases, the cylinder-efficiency decreases, at accelerating rate, until the resultant thermodynamic efficiency, at first ascendant, becomes stationary and finally declines. A temperature-range a little beyond that at which the latter is a maximum dictates the limits for the proper use of simple expansion in a single cylinder.

If any higher efficiency than that just described is desired, compounding must be resorted to. But it is immediately evident that compounding under a total temperature-range and pressure-range (between boiler and exhaust) which gives a maximum efficiency in a single cylinder must 
result in a loss rather than a gain; for compounding is expensive, not only in cost of construction but in incidental losses of heat as well. The steam cannot be transferred from one cylinder to another without distinct loss, in friction and in radiation. Even at a moderate advance of temperature-range over the point just mentioned these losses must overbalance the gains due to

(I) The increased theoretic efficiency, and

(2) The increased cylinder efficiency

due to dividing a greater total temperature-range into two portions, each of which is smaller than that of the simple engine. The first conclusion to be observed, therefore, is that compounding cannot be profitable except under a total temperature-range decidedly greater than that specified as profitable for a single cylinder on page 228. This is the same as saying that compounding cannot possibly be profitable under less than I25 pounds of boiler-pressure when non-condensing, nor under less than 90 pounds condensing.

That is the lower limit. The upper one is probably about I75 pounds when condensing, or about 250 pounds when non-condensing. For it is not until these figures are surpassed that the temperature-range in a single cylinder becomes great enough to warrant the introduction of a third stage of expansion. Where the latter policy has been adopted under pressures similar to the above it has either been for mechanical reasons, as in the marineengine, or in error of judgment, as has come to be generally admitted in connection with triple-expansion stationary engines.

It is therefore obvious that in the design of a compound engine, in the choice of boiler-pressure under which it should work and of cylinder-ratio to be adopted, there is 
the widest possible field for variation of individual judgment. There will be found a correspondingly wide variation in existing practice. In general the problem of the determination of exact cylinder-dimensions would be attacked somewhat in this way.

Problem: To design a compound engine of 100 horsepower normal load, to operate under a steam-pressure of not over 150 pounds and to exhaust into a vacuum. It is assumed that the general type of engine, the speed, the valve-gear, and all similar points outside of thermodynamic ones are already determined; that the engine is to be of the four-valve, receiver type, and to run at 100 revolutions per minute. What should be the cylinder-dimensions?

According to the rule stated on page 244 , the size of low-pressure cylinder must first be determined as if there were to be no high-pressure cylinder present, the full degree of expansion taking place supposedly in the single cylinder. Since condensation and reëvaporation so modify the true adiabatic process that its pressure-volume representation closely agrees with the curve

$$
P V=\text { a constant, }
$$

it is common practice to adopt this as the basis for the projection of indicator-cards in such work as this; partly because the entire problem can never be exact, in either statement or solution, and partly because the simplicity of the curve lends itself to the rapid investigation of the several supposed cases which usually need comparison.

It would first be decided that if 150 pounds by gage were to be the normal boiler-pressure, I 45 pounds by gage or I6o pounds absolute are all that could be expected as the initial pressure within the cylinder, considering variations in boiler-pressure and pipe-friction. Next, the most advantageous terminal pressure would be chosen; say at 
8 pounds absolute. This establishes the total ratio of expansion at $\frac{160}{8}=20$. Let it be assumed that the vacuum to be expected in the cylinder is 25 inches, or $12 \frac{1}{4}$ pounds below atmospheric pressure, or $2 \frac{1}{4}$ pounds absolute.

Referring to Fig. 42, the expansion to the final terminal pressure at $y$, when exhaust into the condenser takes place, would develop the work-diagram $C B D y y^{\prime}$. Of this, the area $B D y F$ is given by the formula (deduced for this curve

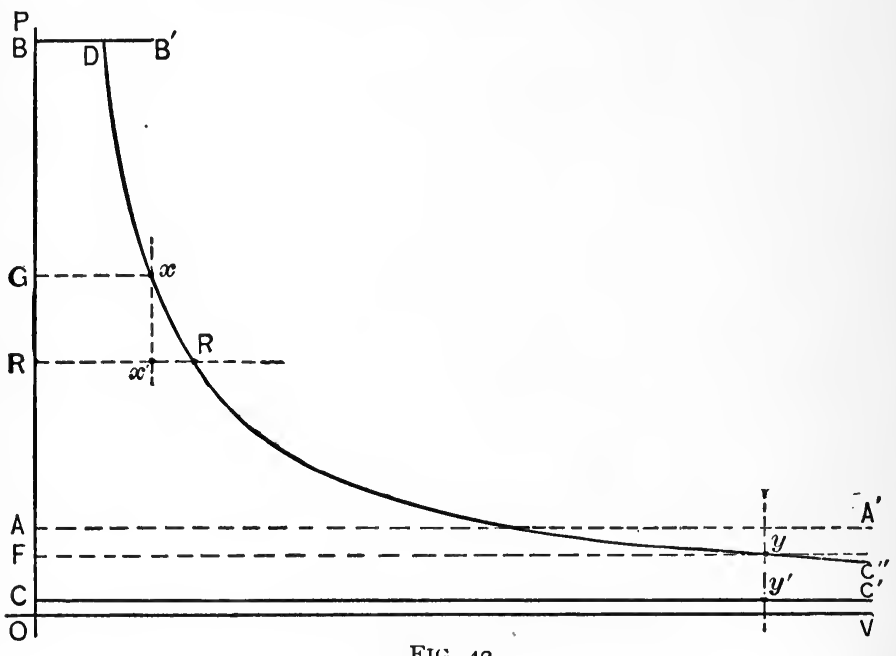

FI(3. 42

on page 122): Work $=P_{1} V_{1} \log r$. The final volume attained by the steam, at the end of the stroke in the largest cylinder, will be represented by $F y$. Let this be represented by $V_{1}$, in cubic feet. Then, since $P_{1}=8 \times 144=$ I 52 pounds per square foot and $r=20$, the work represented by the area $B D y F$ must be I I $52 V_{1} \log _{e} 20=345$ I $V_{1}$ foot-pounds. The work represented by the area $C F y y^{\prime}$ is $\left(8-2 \frac{1}{4}\right)$ I $44 V_{1}=828 V_{1}$ foot-pounds. The total is

$4279 V_{1}$ foot-pounds. 
Since the engine is to be of Ioo horse-power, the work done per minute must be 3,300,000 foot-pounds. Placing this equal to the expression just found and solving for $V_{1}$, gives the value of the latter as 77 I cubic feet. This must be the volume displaced by the piston each minute. If the engine is to make 100 revolutions, or 200 strokes, per minute, the displacement per stroke must be 3.85 cubic feet.

This volume may be attained by any proportion of diameter to stroke which suits the mechanical sense of the designer. The chief guide in the choice is the speed: the slower the speed, the longer the stroke; the higher the speed, the shorter the stroke. A good proportion for this case would seem to be given by a cylinder $17^{\prime \prime} \times 30^{\prime \prime}$, which gives slightly more than the desired displacement. ${ }^{1}$

Receiver-pressure and Cylinder-ratio. - Next arises the question as to the size of the high-pressure cylinder. This is determined by the choice of receiver-pressure and of terminal pressure in the high-pressure cylinder.

The simplest solution of the case is to consider that the total ratio of expansion is to be divided equally between the two cylinders; that is, that the square root of the total ratio is to be the ratio in each cylinder. In Fig. $42 R R$ is the receiver-pressure and $x x^{\prime}$ the exhaust-line at the termination of the high-pressure stroke. By the above argument the pressure at $x$ would be

$O G=O F \sqrt{\frac{O B}{O F}}=\sqrt{O F \times O B}=\sqrt{8 \times \mathrm{I} 60}=35.8$ pounds.

Similarly,

$O R=O C \sqrt{\frac{O B}{O C}}=\sqrt{O C \times O B}=\sqrt{2.25 \times \mathrm{I} 60}=19$ pounds,

${ }^{1}$ It is to be remembered that no consideration is being given here to the effects of clearance and cushion upon the ratio of expansion. Their inclusion would probably bring the figures up to about $18^{\prime \prime} \times 30^{\prime \prime}$. 
or the receiver-pressure would be 4.5 pounds by gage. The volume at $x$ is

$$
G x=F y \cdot \frac{O F}{O G}=F y \cdot \frac{8}{35.8}=F y \cdot \frac{\mathrm{I}}{4.475} .
$$

It is stated in this way because 4.475 is therefore the desired ratio between the volumes of the two cylinders; but as the strokes of the two are nearly always the same, the ratio of diameters may be taken as the square root of the ratio of volumes. This gives, for the diameter of the highpressure cylinder,

$$
\text { I } 7 \div \sqrt{4.475}=8.036 \text {, or, say, } 8 \text { inches. }
$$

This gives, as the proper cut-off in the low-pressure cylinder, $R R \div F y=O F \div O R=22 \%$, and in the highpressure cylinder $B D \div G x=O G \div O B=22 \%$.

Another common rule for determining the receiver-pressure is to divide the work done equally between the two cylinders.

The total work was found to be $4279 V_{1}$ foot-pounds per minute. Half of this is $2140 V_{1}$ foot-pounds. Deducting the area $C F y y^{\prime}$, the remainder of the low-pressure area, $F R R y$, is I3I2 $V_{1}$ foot-pounds per minute. Since this is equal to $P_{1} V_{1} \log r$, and since $P_{1}=8 \times 144, r$ is easily found to be equal to 3.125 . $O R=8 \times 3.125=25$ pounds absolute, as compared with 19 pounds by the former method.

The area remaining for the high-pressure cylinder to develop would be $R B D R$. Some triangular portion of this, as $x R x^{\prime}$, would properly be cut-off in order to give that cylinder an appreciable and profitable terminal pressure, and would be lost; but as it is small compared to the whole, and as the rating of 100 horse-power taken as a basis for all of the calculations is a purely nominal one, 
such incidental minor losses are usually neglected for the time, and are allowed for in lump by increasing the calculated cylinder-dimensions by the fractions necessary to bring them up to some convenient integral figure. Thus, if 20 pounds be chosen as a proper high-pressure terminal, the total pressure at $x$ would become 45 pounds, and the cylinder-ratio would be $F y \div G x=O G \div O F=45 \div 8=5.625$, which calls for a high-pressure cylinder about $7 \frac{3}{16}$ inches in diameter.

But any and all of these methods, of which there are several, are rule-of-thumb substitutes for the only rational thermodynamic one, viz.: the equal division of temperature between the cylinders. In the present case the total temperature-range, from 160 to $2 \frac{1}{4}$ pounds absolute, is $232.8^{\circ}$. Half of this is $116.4^{\circ}$, which, added to the backpressure temperature in the low-pressure cylinder, gives $247^{\circ} \mathrm{F}$. as the proper receiver-temperature, or 28.3 as its proper absolute pressure. A high-pressure net terminal of 20 pounds again would make the gross high-pressure terminal at $x, 48.3$. The cylinder-ratio would be $\frac{48.3}{8}=6.04$. The diameter of the high-pressure cylinder would be $17 \div \sqrt{6.04}=6 \frac{15}{16}{ }^{\prime \prime}$; say 7 inches.

It is obvious that all of these methods lead to very similar conclusions. The second one is very commonly resorted to, originally because it was very easily carried out by means of the indicator. It rests upon good foundation in those cases where mechanical considerations outweigh thermodynamic ones; but the false idea has grown out of this practice, and been widely taught to operating engineers, that the equal division of load between the cylinders is thermodynamically proper. It is usually mechanically proper, though sometimes not. It is only a coincidence if the thermodynamic results happen to be good. 
Clearance and Cushion. - The influence of clearance and cushion upon capacity, or upon ratio of expansion, has been. openly excluded from the above calculation. The method of allowing for it was explained in Chapter I, page 196. Suppose that in the low-pressure cylinder of the above engine it be known that the actual clearance (which would be estimated from a knowledge of the type of valve-gear) would be about $2 \%$. The calculations on page 196 showed that such a clearance necessitated the increase in volume of the cylinder, in order to get the same results from the same quantity of steam, from 2.62 to 2.77 cubic feet, or by about $6 \%$. Such an allowance for clearance as this would call for the increase of the 17 -inch cylinder to $17 \frac{3}{4}$ inches in diameter. The high-pressure cylinder should of course be similarly increased in diameter, to correspond.

The preceding problems cover the bulk of what may be stated with mathematical accuracy regarding the choice of cylinder-ratio in compounding. In the remaining portion of the field, which is open to variation of opinion, there is the widest divergence between the judgments of men of the highest standing. The Westinghouse compound stationary engine and the Vauclain compound locomotive, both of which have been exceptionally successful designs, use a ratio of about 2.7 to I under as high as 200 pounds boiler-pressure, but with no vacuum. Mr. George I. Rockwood advocates 7 to $I$ as a proper ratio, under even lower boiler-pressures when exhausting into a vacuum, and his engines have given good results. Between these two extremes might probably be assorted all the different opinions regarding compound-cylinder ratios in the country. It would seem unquestionable, however, in spite of this wide divergence of opinion, that a broad survey of the progress of experience in engine-design reveals the following facts : 
(I) That the steam-pressure which can be efficiently expanded in two stages is higher than was formerly supposed. The need for the intermediate cylinder in the triple compound is based upon purely mechanical, not thermodynamic, principles. The maximum cylinder-efficiency is attained in each cylinder of a compound engine under approximately the same conditions as in the cylinder of a simple engine, viz.: under from four to six expansions. These three statements are practically synonymous.

(2) That, as a result, cylinder-ratios are steadily upon the increase.

The favorite ratio in England was, for many years, for double compounds four to one and for triple compounds six to one, as between low- and high-pressure cylinders. This applied normally to large condensing compounds. Compounding was adopted more reluctantly in America and at first copied English practice in this respect. By I895, however, the plan of carrying a standard line of compound designs and patterns for all services was well established. As the majority of these engines were sold to operate either under steam of abnormally low pressure from existing boilers, or under atmospheric exhaust, the ratio of four to one was found to be too large. General practice soon settled down to the region between 2.75 and 3.25 to one. Within the past few years, however, the trend is back toward the higher ratios, and values above four to one are now quite common in new engines from the best works.

To the marine engine, where mechanical considerations outweigh thermodynamic ones, these remarks, of course, do not apply. Cylinder-ratios are much lower there, in order to avoid too early cut-offs and too heavy cushion for engines without fly-wheels. 


\section{The Receiver-TyPe AND THE Woolf-type of}

Compounds

It is almost universal practice, in compounding, to exhaust from the high-pressure cylinder into a chamber of considerable volume, called a receiver, and to draw from it the supply for the low-pressure cylinder as from a boiler. The advantages resulting from this plan are several. They may be lumped under two heads, as follows :-

(I) Mechanical. - The timing of the motions of the two pistons is quite independent one of the other. The cranks driven by the two cylinders may be set at any desired angle.

(2) Thermodynamic. - The pressure in the receiver remains nearly constant; hence the temperature-range in the high-pressure cylinder is only that from boiler to receiver temperature, and the temperature-range in the low-pressure cylinder is only that from receiver to exhaust temperature, Were this not so, were there a variation in the receiverpressure, the high-pressure temperature-range would extend from boiler to the lowest receiver-temperature, while the low-pressure range would be from the highest receivertemperature to exhaust. The two ranges would lap, their sum would exceed the total range from boiler to exhaust, and the resultant condensation would be greater than it otherwise might be.

This is the condition of affairs in the Woolf-type of compound. It has no receiver. The two cylinders are separated only by the exhaust and admission valves. This necessitates -

(I) That the pistons must move simultaneously.

(They may move in opposite directions, however.)

(2) The intake of the larger cylinder can consist only of the exhaust from the smaller; the difference in size be- 
tween the two pistons therefore insures that the "receiverpressure" must steadily decrease, owing to expansion, during the exhaust-stroke of the high-pressure and the admission-stroke of the low-pressure cylinder. These phenomena are visible in Fig. 43, in which the dotted lines show the effect of shortening the cut-off from that shown by the full lines. ${ }^{1}$

The Woolf engine was one of the earliest compounds to

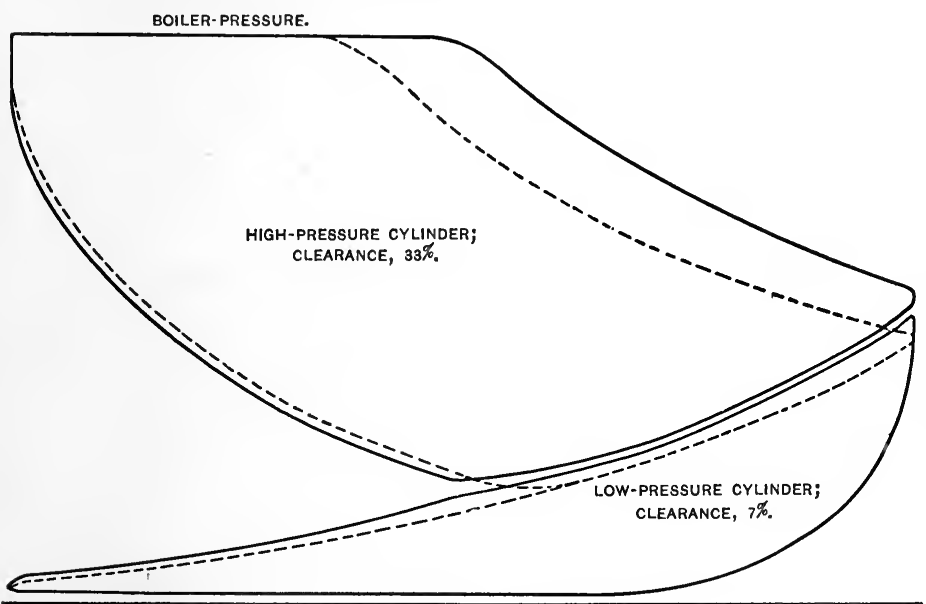

ATMOSPHERIC PRESSURE.

FIG. 43

appear, late in the eighteenth century. Unable to successfully compete with Watt's monopoly or to establish its superiority under the low steam-pressures then prevailing, it failed and disappeared. Within the last twenty years, however, its marked superiority under certain conditions has been appreciated and it has reappeared.

For the reasons just given, the receiver-type of com-

1 See Trans. Am. Soc. M. E., Vol. XIII, "Steam-distribution in a Singleacting Engine," F. M. Rites. 
pound is superior, both in mechanical adaptability to diverse arrangements of piston-motion and in thermal efficiency, to the Woolf-type, under nearly all conditions of service. It is therefore only necessary, in order to properly define the worth of the latter, to consider the conditions under which the receiver-type is not a success. These may be very easily and narrowly defined as a light or variable load combined, with atmospheric exhaust. Under these conditions the efficiency of the receiver-type diminishes so very rapidly as to easily fall below that of the simple engine, and sometimes below the poorest which could ever be expected from a good simple engine.

The reason for this is that under light loads the governor admits to the engine so small a volume of boiler-steam that the terminal pressure in either or both cylinders falls below the exhaust-pressure. The result is a loop at the lower end of the card, the pressure in the cylinder rising when the exhaust-valve is opened, instead of falling, as it should. While a loop at the upper corner of the indicator-card signifies little loss of efficiency, if any, a loop at the lower end of the card is trebly bad.

(I) It much exaggerates the evils already described as arising from complete expansion to exhaust-pressure, on a sharp-toed diagram.

(2) It absorbs power excessively which is not returned to the engine in the slightest appreciable degree.

(3) It exaggerates cylinder-condensation by sucking in through the exhaust-port moisture which, much of it, must reëvaporate within the cylinder at the expense of heat made good from the next initial condensation.

Trouble (2) could theoretically be cured by equipping the cylinder with check-valves opening back from exhaust into the cylinder whenever the pressure in the latter fell too low; but they would be noisy, frail, and troublesome, 
and would have no effect on the other evils. They have never been widely applied to mill-engines, though common in locomotives.

The effect of these losses is pernicious in the extreme. In one case it was reported, by a high authority, that a good specimen of Corliss compound engine, new and in good condition, which would ordinarily be expected to develop a horse-power upon about I 4 to I 5 pounds of steam per horse-power-hour when properly loaded and supplied with a vacuum, actually consumed, when lightly loaded and exhausting into the atmosphere, 72 pounds of steam per horse-power-hour. If the low-pressure cylinder had been disconnected, the other cylinder alone would have developed the power, under these conditions, at a rate not much exceeding one-third of this figure. It did develop about I 80 horse-power at just about that rate; but the lowpressure cylinder, acting as a brake by reason of its having to drive its exhaust out against an atmospheric pressure which was higher than its own terminal pressure, absorbed over Ioo horse-power, so that the net rate was that stated. The high-pressure cylinder alone would not, of course, have developed the 70 or 80 net horse-power as efficiently as it did the I 80 horse-power; but the rate, even so, could hardly have risen above 30 pounds per horsepower-hour.

The superiority of the Woolf-type under these same conditions is due to the following circumstances :-

There being no volume between the two cylinders, any cut-off in the low-pressure cylinder must also act as the closing of the exhaust of the high-pressure cylinder, or as the point of compression in that cylinder. The fact that an ordinary cylinder cannot be given more than a certain degree of cushion (without exceeding boiler-pressure before admission takes place) has led to the oft-stated but false 
idea that the low-pressure cylinder of a Woolf compound could have no cut-off. But any cylinder may be given as early a cushion as is desired, provided there be sufficient clearance to absorb the compression before too high a terminal pressure is reached. Therefore all modern designs of Woolf-type compounds are provided with an unusually large clearance in the high-pressure cylinder. This permits an early cut-off in the low-pressure cylinder and an early, slowly rising cushion in the high-pressure cylinder.

If this cushion were sufficient to engender boiler-pressure in the clearance space before admission took place, and if there were no thermal losses to cylinder walls, this large clearance would involve no loss of efficiency (see page 206; 236). In practice neither condition is supplied. Losses are involved from both sources. The large clearance must be partially refilled with boiler steam before each stroke commences; owing to thermal interchanges between the steam and walls the cushion-steam does not return as much work during expansion as it absorbed during compression. Therefore the Woolf-type never attains the maximum efficiency usual in receiver-compounds when run under proper conditions. Nevertheless, the sum of these two losses in the Woolf-type is not nearly so great as is that incurred by the over-expansion of the receiver-type under light loads and no vacuum. The latter loss can be entirely avoided in the Woolf-type by making the high-pressure clearance sufficiently large, so that under the lightest possible load, zero load, say, for an extreme illustration, - there will always be steam enough in the engine to expand through both cylinders and still be above atmospheric pressure at the end of the low-pressure stroke. Since the cushions in both cylinders are supposedly so designed that the ordinary back-pressure, while the exhaust is open to the atmosphere, is compressed back to boiler-pressure in the high-pressure 
clearance, there would be, theoretically, in the extreme case of no load just supposed, no intake of steam from the boiler at all. In practice this nice adjustment of valve-motions is found impracticable, and some steam is always consumed in merely refilling the high-pressure clearance at each stroke; but the approach to the theory is quite close.

The origination of this plan of operation the world owes to Mr. F. M. Rites, who incorporated it into the design of the Westinghouse compound engine, which is a pure Woolf-type engine. It is also embodied in modified form in the four-cylinder Baldwin compound locomotives of Mr. Vauclain's design. Since this beginning of its revival other designs of Woolf-type engines have been placed upon the market.

\section{Methods of Steam-distribution}

Compound engines may be rigged with any of the several types of valve-gear used on simple engines: The single balanced-slide or piston-valve, driven either by a fixed eccentric (with throttle governor), by a shaft-governor, or by link-motion; the four valves of the Corliss engine or their equivalent, closed either by drop cut-off gear or positively; or the double slide or piston-valves of the Myers-type. But the differences between these gears are much the same in compound as in simple engines. Their discussion belongs to a study of valve-gears, not that of the thermodynamics of compounding.

There is one question calling for decision in such work, however, which has direct thermodynamic influence upon the efficiency. That is : Shall the cut-off in the low-pressure cylinder be varied by the governor as the load changes, or shall it remain fixed?

There is much needless discussion of the question, chiefly 
because of doubt as to what result is desired, rather than as to what will ensue from a given course of action. For it is plain that if the low-pressure cut-off remains fixed while the steam admitted to the high-pressure cylinder is varied with the varying load, the receiver-pressure must also vary with the load. When the latter is heavy the receiver-pressure will rise; when the load is light, it will fall. If, on the other hand, the cut-offs of the two cylin-

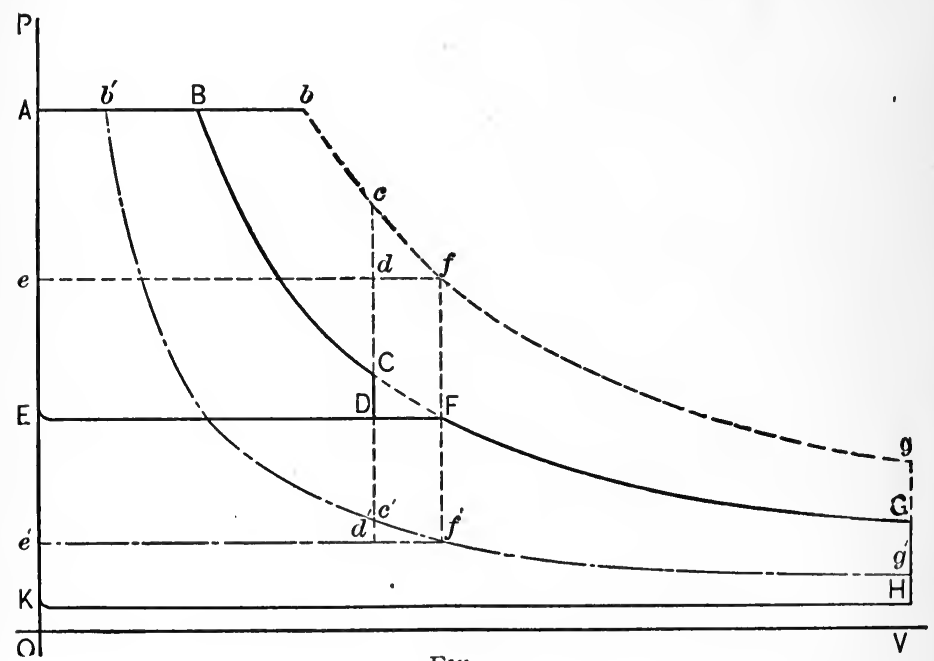

FIG. 44

ders were varied together and equally by the governor as the load altered, the receiver-pressure would remain practically constant.

I. Low-pressurc Cut-off Fixed. This situation is shown by Fig. 44, wherein, under normal load, the governor admits the volume $A B$ of boiler-steam to the engine. $A B C D E$ is the high-pressure indicator-card and $E F G H K$ is the lowpressure card.

When the load increases, the governor admits the volume 
of boiler-steam $A b$. Its curve of expansion is $b c f g$. The high-pressure card becomes $A b c d e$ and the low-pressure efg $H K$.

Under lighter loads the expansion-curve becomes $b^{\prime} c^{\prime} f^{\prime} g^{\prime}$ and the receiver-pressure falls to $e^{\prime} f^{\prime}$. The high-pressure card becomes $A b^{\prime} c^{\prime} d^{\prime} e^{\prime}$ and the low-pressure card becomes $e^{\prime} f^{\prime} g^{\prime} H K$.

II. Low-pressure Cut-off always Equal to High-pressure Cut-off. This situation is shown in Fig. 45. The normal-load

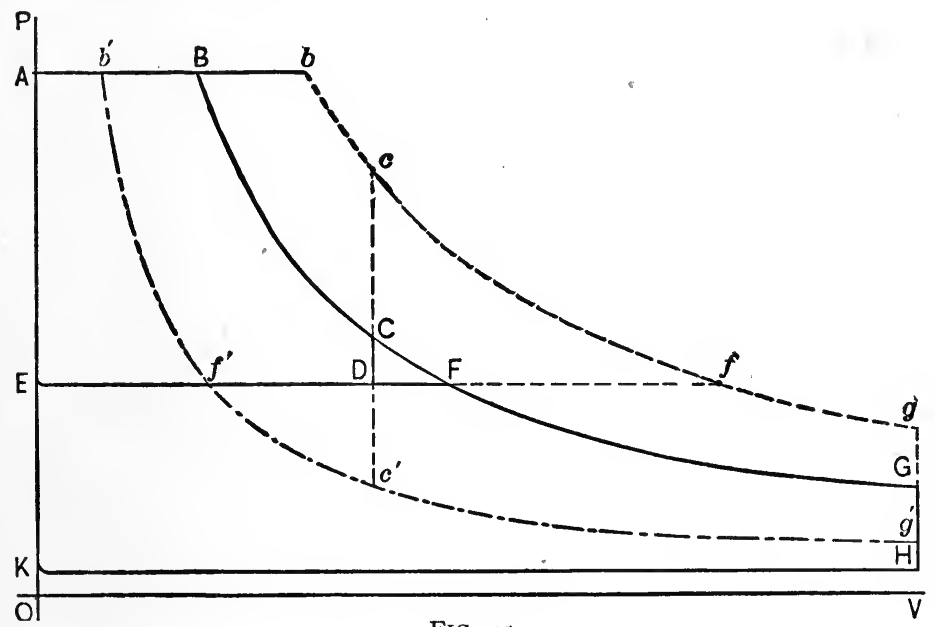

FIG. 45

diagrams are $A B C D E$ for the high-pressure and $E F G H K$ for the low-pressure, as before.

An increase in load moves the expansion-curve out to $b c f g$, as before; but because the low-pressure cut-off has lengthened to $f$ the receiver-pressure does not rise. The high-pressure card becomes $A b c D E$. The low-pressure card becomes EfgHK.

Under lighter loads the expansion-curve becomes $b^{\prime} f^{\prime} c^{\prime} g^{\prime}$; but again, because the low-pressure cut-off has shortened 
from $F$ to $f^{\prime}$, the receiver-pressure remains the same. The high-pressure card becomes $A b^{\prime} f^{\prime} c^{\prime} D E$. The low-pressure card becomes $E f^{\prime} c^{\prime} g^{\prime} H K$.

Comparative Efficiency. - The chief differences between the two plans are obvious. The primary advantage of the first is that under all loads the small triangular area $C D F$, which represents receiver-loss, remains practically constant. It is entirely under control and is fixed in the original design of the engine and the set of the low-pressure cut-off. If the volume in the low-pressure cylinder at the instant of cut-off were equal to the cylindrus of the high-pressure cylinder, this loss-area would be zero under all loads; by making the high-pressure cylinder a little smaller than this, some cost and friction can be saved, at the expense of no appreciable loss of power or efficiency, and the receiverdrop $C D$ can be made whatever is desired.

In the second plan, on the other hand, the receiver-loss varies widely. Under heavy loads it grows to $c D f$. As the load falls below normal it decreases first to zero and finally becomes negative, an extreme which is far more harmful to efficiency than its opposite. The terminal loss at $G H$ or $g H$ is alike in either plan. So that the thermodynamic advantages lie all with the first plan.

In respect to mechanical features, however, it is quite otherwise. Under the second plan the work is divided between the two cylinders quite evenly under all loads. Under the first, on the other hand, heavy loads are developed almost entirely in the low-pressure cylinder and light loads almost entirely in the high-pressure. This is mechanically bad. It could be allowed for, however, by proper design and construction. But this distribution of power has a secondary and very detrimental effect upon another important feature of engine-operation, viz. :-

Regulation. - Under the first plan the receiver-pressure 
is varied up or down by the constant volume-pull of the lowpressure cylinder upon a varying supply of steam to the receiver from the high-pressure cylinder. The receiverpressure will therefore be constant after any alteration of load, only after these two factors have had time to attain a condition of equilibrium. In other words, when the load decreases, the low-pressure cylinder finds itself supplied with steam of a much higher pressure than that of equilibrium. It therefore develops more power than it would after the steady conditions which are normal to this load have been attained. But, the load having fallen off, this is just the time when less power, not more, is wanted from the low-pressure cylinder. The engine therefore jumps above speed until the excessively short high-pressure cut-off induced thereby, coupled with the heavy draft of the lowpressure cylinder on the receiver, has brought the pressure of the latter to the proper point.

But the process cannot stop there, for equilibrium is not yet attained. The engine-speed is above normal ; the highpressure cut-off is shorter than that suited to the load. The receiver-pressure therefore continues to fall below that of equilibrium until arrested and brought back by a reversal of the entire above process, which will be instituted only when the engine-speed has again fallen below normal, a thing which must finally result from the too short cut-off in the high-pressure cylinder.

This process of wavering above and below normal speed at every change of load is called "hunting." There are a long list of causes of hunting which lie in the mechanics of the governor. But the effect of all such causes will be much exaggerated by compounding with fixed low-pressure cut-off. Indeed, so extreme is this exaggeration in some cases that there is no position of equilibrium possibly to be found; the engine surges above and below normal speed 
so violently that operation under loads which vary appreciably is quite impossible.

Extreme conditions may dictate the adoption of either plan unreservedly. That is, extreme demand for perfect regulation may enforce the full variation of low-pressure cut-off with that of the high-pressure cylinder, regardless of the effect upon the efficiency; or the need for the most refined efficiency may call for the abandonment of all hope of close regulation. Usually, however, a compromise is wise. The attachment of the low-pressure cut-off to the governor will insure moderately close regulation, but by diminishing the extent and rapidity with which it is varied by the governor a good grade of efficiency is maintained.

Reheaters. - It will be remembered, from the theory of thermodynamics, that the adiabatic expansion of saturated steam must always develop moisture. Since all the thermal effect of the cylinder-walls must be to produce considerable moisture before expansion begins, and since comparatively little reëvaporation takes place before the exhaust-valve opens, there is always a considerable percentage of water thrown into the receiver from the highpressure cylinder.

In many engines, particularly where the importance of constructive simplicity outweighs thermodynamic considerations, the presence of this water is entirely neglected. In the greater number of large stationary engines, however, the receivers are drained of this water with more or less care, by traps or by hand. In this way the receiver acts as a separator upon the passing steam; but as scarcely any separator can be expected to deliver steam containing less than one or two per cent of moisture, on the average, there is still an appreciable amount of moisture which finds its way into the low-pressure cylinder, where it operates to exaggerate condensation in the manner already described. 
The thermodynamic disadvantages of this condensation and the corresponding advantages of using perfectly dry, or even superheated, steam, coupled with the facility with which such moisture can be dried out by means of coils of pipe filled with boiler-steam inserted in the receiver-volume, has led to the wide adoption of such a plan in engines of considerable size which aim at the best thermodynamic efficiency. Since the temperature within the coils is much higher than that of receiver-saturation the conduction of heat to the steam is comparatively rapid; at any rate, until all moisture has disappeared. The cost of the engine is slightly greater with the added coils, but that of operation is decreased. The trap which would have been used to drain the receiver can be used to drain the coils. The receiver-steam always enters the low-pressure cylinder dry; sometimes it is considerably superheated. The gain in efficiency is always marked. It varies so widely, however, with variations of service, size of receiver and coils, etc., that no rule can be given, either for the volumes or heating-surface to be chosen or for results to be expected.

The Steam-turbine. - The applicability of thermodynamic theory to the action of steam-turbines is very limited. This action is, theoretically, upon the Rankine cycle. It is often, and most profitably, modified by superheating the steam. In this case, however, arises the difference between the turbine and the piston-engine that in the latter the superheat seldom succeeds in penetrating the cylinder until cut-off occurs, and in so influencing expansion; while in the former, since reciprocation and its corollary, condensation and reëvaporation, are wanting, the steam actually operative within the engine is superheated.

The essential process of the steam-turbine is the preliminary conversion of the steam-heat into kinetic energy of translation before its absorption by the machine. If 
this process occupy the entire temperature-fall available, the resultant velocity of translation is excessively high; and since the velocity of the vane which is to absorb its energy should be a fair fraction thereof, this calls for excessively high speeds of rotation. To avoid this the temperature fall is usually split up, or compounded, into a great many steps, each of which develops only a moderate velocity, which velocity is absorbed by moving vanes before the next step is undertaken.

But velocity cannot be developed without concurrent friction. The adiabatic conversion of the heat into kinetic energy is therefore no sooner undertaken than frictional losses enter, tending to divert the path of fall from the vertical adiabatic toward the constant-heat curve (Fig. 14). The besetting sin of the piston-engine, on the other hand, it may be noted, is the departure of the path of fall from the adiabatic in the other direction, into the southwest quadrant, by condensation.

The questions arising as to the proper limits for the development of velocity before the frictional losses become too great, and as to the best form of vane, of wheel, etc., for absorbing the velocity developed, are problems in the mechanics of fluids rather than in thermodynamics. 


\section{CHAPTER III}

\section{THE OTTO GAS-ENGINE}

THE variation of the actual cycle of the Otto-type of gasengine from the theoretic is much more difficult to trace than is that of the steam-engine. More observations of a much more difficult character go to the organization of a gas-engine test than to that of a steam-engine test. The data which are most essential to the complete thermodynamic analysis of the processes occurring within the cylinder cannot be gotten at all, by any means yet adopted by the profession; assumptions and estimates based upon what observations it is possible to make must be substituted therefor. For instance, one of the most important observations needed, that of the temperature of the uncompressed charge of explosive mixture in the cylinder at the beginning of the compression-stroke, has never been taken but once or twice and then at a great cost of money and effort.

The prime factors in the modification of the actual gasengine cycle from the theoretic are the following. They are named about in the order of their importance.

(I) The abstraction of the heat from the burning gases by the water-jacket.

(2) Heat-interchanges with the cylinder-walls similar to the condensation and reëvaporation of the steam-engine.

(3) The influence of variations from the normal cycle introduced in order to modify the power developed.

(4) The slowness of inflammation.

(5) Heat-abstraction by the water-jacket during compression. 
Of these, numbers (I), (3), and (4) only are visible in the indicator-card. The others become graphically visible only in the entropy-temperature diagram. They can all of them, of course, be calculated algebraically.

In Fig. 46 let the area $B C D E$ represent the theoretic possibilities of an Otto gas-engine in the same way as did

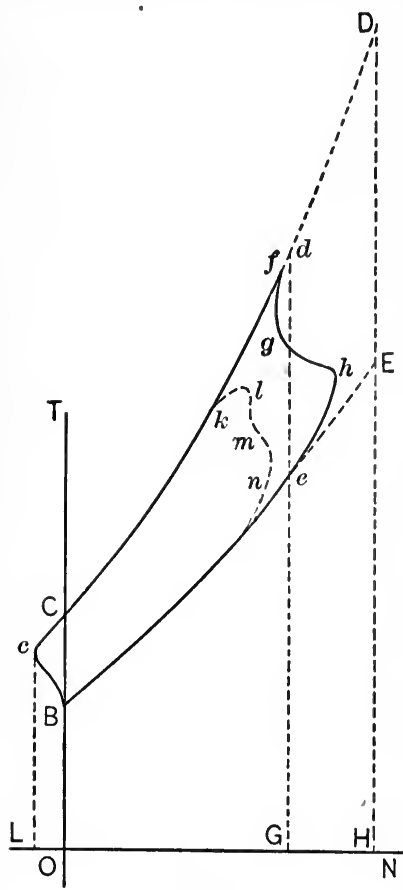

FIG. 46

Fig. 2I, page I40. If the corresponding pressure-volume diagram, Fig. 22, were reproduced here and the indicator-card from the actual engine superimposed upon it, there would be found wide discrepancies between the two. It is not worth while to do this, however, because a comparison between theoretic and real pressure-volume diagrams does not illustrate differences in heat-energy, which is the thing needful, but only differences in their effects; and as analysis is undertaken only for the purpose of detecting causes, recourse must be had to the entropy-temperature diagram. The method of doing this mathematically will be explained on page $28 \mathrm{r}$. In the meantime let attention be turned to what happens within the cylinder.

During the process of compression, which theoretically passes adiabatically from $B$ to $C$, heat is abstracted by the water-jacket. The actual path of compression therefore departs to the left and follows some course similar to $B C$. Then ignition and inflammation take place and temperature 
and entropy increase along $c d$. The heat supplied is sufficient to carry the explosive development of heat to $D$. While the process is in operation, however, the waterjacket steals some $35 \%$ to $50 \%$ of the total heat supplied, or the area $G d D H$; the isomorphic development of heat therefore cannot pass beyond $d$. By observation of the heat absorbed by the jacket-water during the test of the gasengine in question the point $d$ may be determined on the theoretic diagram. But the abstraction of heat by the waterjacket does not occur in such a way as to take the simple form $G d D H$, leaving the actual cycle to be $B C d e$. Some of it occurs during compression, and is measured by $O B c L$.

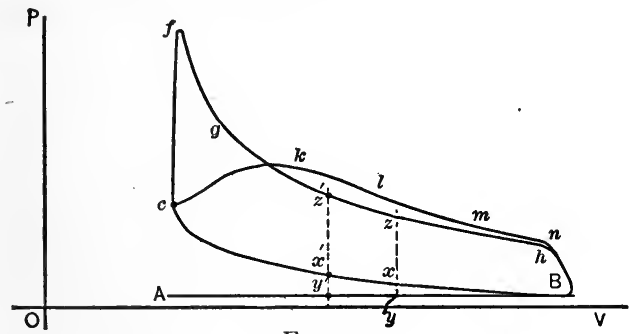

FIG. 47

But the greater portion occurs during explosion and the first portion of expansion, and is measured by $H D f g h e G$, in which the line $e G$ now takes such position as will make this area and $O B C L$ together equal the measured loss of heat. For this reason the limit of explosive rise of temperature, $f$, never quite coincides with the point $d$.

Standard gas-engine diagrams may be divided into two sorts or classes, one from each of which is shown in Figs. 46 and 47. The first is the true standard card of maximum efficiency under a given degree of compression and quality of gas; it is shown by BcfgheB. The other is what very often appears in its place, but which is usually due to no more serious defect than a lack of proper adjustment. It is 
shown by $B c k l m n B$. In it inflammation takes place too late or too slowly. It is always due to one of two causes:

(I) Late ignition;

(2) Mixture of gas and air in improper proportions. As to its effect upon the efficiency, the pressure-volume diagram reveals nothing certain; because it is not obvious, from a single diagram such as cklmn, Fig. 47, whether the area developed would be greater or less than that which would otherwise have been produced had inflammation been more prompt, such as $c f g h$. Or, if comparison by measurement be instituted, there is no argument whereby the result can be known to apply to other cases. But in Fig. 46 it is plain that any departure of the piston upon its course before inflammation is complete must result in work being done and hence in the bending of the curve $k f$ around to the right toward first the horizontal isothermal and beyond that toward the vertical adiabatic, into some such path as $k \mathrm{~lm}$. This is fatal to the attainment of the best efficiency. It is evident without further inspection that nothing which the cycle may do later in its course may hope to make good this loss of availability for work.

The transition from the isomorphic of to the approximated vertical adiabatic of expansion (Fig. 46) is usually and properly in the form of a quite acute cusp, as at $f$. Otherwise it is more rounded, as along $\mathrm{kl}$. Sooner or later, however, the transition is made. It is then to be particularly noted that the change of direction always swings beyon 1 the vertically downward, or true southerly, direction into the southwesterly quadrant. This shows that the development of heat within the working-substance is replaced by an abstraction of heat. The only exceptions to this rule are in occasional cards in which the first portion of the expansion-line, $f g$, of a sharp-pointed card is truly vertical. So that even these exceptions show that 
the development of heat has come sharply to an end and that for the moment no heat-interchanges are taking place whatever.

Considerable emphasis must be laid upon this point, because for many years much has been said and written about "delayed combustion " or " afterburning," and about dissociation as one of its causes. Since the basis for all such arguments has always been the form of the expansioncurve, it is important to note that the first thing proved by the form of that curve is that combustion is always complete by the time that maximum pressure is reached; or that if it be delayed it is only in those cases where the incipience of combustion has been delayed. In other words, explosive combustion in an Otto gas-engine, instead of being a dual or complex process, part of it occurring immediately after ignition and part of it delayed by some mysterious cause until late in the expansion-stroke, appears, when seen in the entropy-diagram, to be a simple continuous process occupying a fairly definite amount of time. If it be inaugurated well before the crank reaches dead-centre, it is usually complete by the time the piston has moved appreciably on its outward stroke. If it be not started until that stroke has begun, or if the mixture be a slow one, it may last continuously until the stroke is well toward completion. But there is no sign of its being interrupted, or of its being delayed or postponed by any other causes than those which always lead to the lapse of an appreciable period of time in explosive combustion.

It is next to be noticed that the portion of the cycle just under discussion, which is always convex toward the right, whether in the form of an apparently sharp point, as at $f$, or of a well-rounded curve, such as $k l$, is always followed by a process which is concave toward the right. The only exceptions to this rule which the writer has ever met are in 
those cases where the exhaust-valve opens before combustion is, or could reasonably be expected to be, complete.

The significance of this change of curvature is marked. Convexity toward the right shows the rate of heat-supply becoming steadily less. Mathematically, it might also show rate of heat-abstraction steadily increasing; but consideration of conditions actually prevailing makes this proposition untenable.

Concavity toward the right, on the other hand, necessarily shows active development of heat. Where the concavity has not yet brought the expansion-curve again into the vertical adiabatic there may be some debate of this proposition. Where it has brought the curve into the southeasterly quadrant, however, - and this is nearly always the case, - there can be no question. The departure of the curve to the east shows the addition of heat.

It was this portion of the process which led to the theory of delayed combustion. Because the pressure-volume diagram is very meagre in its information, it was not observed at the same time that this addition of heat is separated from the original development of the fuel-heat of the gas in combustion by a distinct period when no production of heat took place. This intervening period being now known, what explanation of the situation is most probable?

Since the expansion-curve, whether of the form of $\mathrm{fgh}$ or of $k l m n$, is strikingly similar to the expansion-curve of the simple, non-jacketed steam-engine, ${ }^{1}$ and since in the latter case there is no possible source of heat except the cylinder-walls, it is only rational to assume that there takes place in the gas-engine cylinder a reversing transfer of heat between working-substance and walls which is in every way the parallel of the familiar condensation and reëvaporation of the steam-engine cylinder. Such a simple explanation

1 See Fig. 41, page 219. 
covers the demands of all the known facts. It eliminates the unscientific mystery of "delayed combustion." It may be assumed as demonstrated that nothing occurs within the gas-engine between the point of ignition and the opening of the exhaust-valve but the prompt, continuous combustion of the charge, its expansion due to the motion of the piston, and the varying action of the jacketed walls upon it in the ways naturally to be expected.

It is a widespread fault in the commercial and professional treatment of the gas-engine which has led to this doubt and confusion; and against it the student should be warned. I refer to the common practice of making repeated tests of gas-engines at great expense of time, labor, and money, without commensurate effort at an analysis of the results obtained. It is of little use to ascertain the facts unless we learn their causes. Such knowledge of causes cannot be had from the indicator-card direct. The compression and expansion curves of Fig. 49 are dumb as to all the thermal phenomena of which their translations into the curves of Fig. 48 become eloquent. Such translation is simple and fairly expeditious. It is not to be understood why it is not more frequently performed. Its omission is in line with the frequent elaborate reports of elaborate tests, with no mention made of the clearance of the cylinders or of the calorific power of the gas.

The Entropy-temperature Analysis. - In the translation of pressure-volume or indicator diagrams into entropy-temperature curves, the following argument is relied upon:-

Let Fig. 49 be one of the indicator-diagrams, supposedly an average one, of a set taken under constant conditions. Let Fig. 48 be an entropy-temperature coördinate field on which has been located only the initial point $B$.

In the location of this point $B$ only the temperature need be considered. As to entropies, all that is usually desired 
in the entire analysis is a knowledge of comparative alterations, to know whether heat be entering or leaving the working-substance. But even where the quantities of heat leaving or entering are desired, and where entropies are therefore to be taken to exact scale, the zero-point may be located horizontally arbitrarily. As to temperature, however, there is considerable room for discussion. It cannot be known by observation, except at a cost which is nearly always prohibitive. Without it the absolute temperature at any point in the cycle cannot be known. But, on the one hand, much can be learned from the entropy-analysis with only knowledge as to comparative alterations of temperature. On the other, the temperature $B$ cannot be outside of certain fairly definite limits. It cannot be below atmospheric temperature. The engine cannot have raised it above atmospheric temperature by more than a reasonable amount. Finally, since the temperatures after explosion, as calculated from the assumed initial at $B$, will be in error by four or five degrees for every one of error in the assumption at $B$, too great error will be checked out by improbability at either the top or the bottom of the cycle. The writer usually assumes the temperature of $B$ at the convenient round number of $600^{\circ}$ absolute, Fahrenheit. ${ }^{1}$

On the indicator-diagram first lay off the vertical axis $O P$ (Fig. 49) at the proper distance to the left to measure

1 Professor Burstall's measurements show higher temperatures, at all portions of the cycle, than would justify this assumption. The mean of those made for the Gas-engine Research Committee of the Brit. Inst. M. E., upwards of $250^{\circ} \mathrm{F}$. during suction and $3600^{\circ} \mathrm{F}$. during explosion, would point to an assumed initial temperature properly as high as $700^{\circ}$ absolute, instead of $600^{\circ}$. But as these measurements were made with a very small engine, running below speed and missing some five explosions out of six, the rigidity with which they may be applied under more normal conditions must be questioned. The writer's figure, $600^{\circ}$, is undoubtedly as low as the truth. (See Trans. Brit. Inst. M. E., I901.) 
the clearance. Also draw the zero-axis of pressure, $O V$. Then erect any number of ordinates through any desired points whatever. It will save work to carry these ordinates completely across both curves. Then in order to locate upon Fig. 48 any desired point, such as $x$, of Fig. 49 , lay off (in the imagination) from $B$, Fig. 48 , a constantpressure isomorphic $B y$. This must represent geometrically the same process as $B y$ of Fig. 49. Whether any

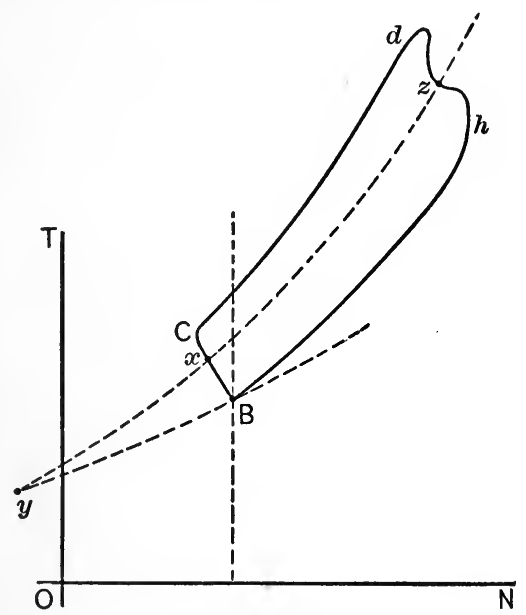

FIG. 48

such process actually took place or not does not affect the mathematical argument. From $y$, Fig. 48, lay off the constant-volume isomorphic $y x z$. This must correspond in the same way to the line $y x z$ of Fig. 49, and upon it may be located both $x$ and $z$.

The temperatures and entropies of the points $y, x$, and $z$ may be had by calculation by remembering -

(I) That on the constant-pressure process By the temperatures must be proportionate to the volumes, which are measurable from $O P$; 
(2) That on the constant-volume process $y x z$ the temperatures must be proportionate to the absolute pressures, which are measurable from $O V$; and

(3) That in any case the equation $\frac{P V}{T}=\frac{P_{1} V_{1}}{T_{1}}$ holds true.

Let the pressures and volumes at the points $B, x$, and $z$ be measured in any convenient scale whatever; a decimal scale will be found the most convenient. Let the ratios $\frac{V_{B}}{V_{x}}, \frac{V_{B}}{V_{x^{\prime}}}$, etc., the ratios $\frac{P_{x}}{P_{B}}, \frac{P_{x^{\prime}}}{P_{B}}$, etc., and the ratios $\frac{P_{z}}{P_{x}}, \frac{P_{z^{\prime}}}{P_{x}}$, etc., be found and tabulated. (By setting a slide-rule to, first, $V_{B}$, and then to $P_{B}$, and going over the entire diagram,

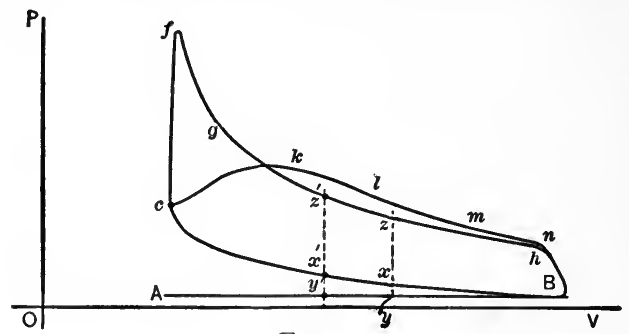

FIG. 49

first for volume-ratios and then for pressure-ratios, this can be done very expeditiously.)

Next, note for each desired point the logarithms of these ratios. If greater accuracy be desired, the logarithms of the original measurements might have been noted, the logarithms of $P_{B}$ or $V_{B}$ being subtracted from them; but this is seldom necessary.

From the equation noted in paragraph (3) above :-

$$
\log \frac{T_{x}}{T_{B}}=\log \frac{P_{x}}{P_{B}}-\log \frac{V_{B}}{V_{x}} .
$$

(See page 120.)

From page 83,

$$
N_{x}-N_{y}=S_{v} \log _{e} \frac{T_{x}}{T_{y}}, \text { and } N_{B}-N_{y}=S_{p} \log _{e} \frac{T_{B}}{T_{y}}
$$


Therefore,

$$
\begin{aligned}
N_{x}-N_{B} & =S_{v} \log _{e} \frac{T_{x}}{T_{y}}-S_{p} \log _{e} \frac{T_{B}}{T_{y}} \\
& =S_{v}\left(\log _{e} \frac{T_{x}}{T_{y}}-\mathrm{I} .408 \log _{e} \frac{T_{B}}{T_{y}}\right) \\
& =2.3026 S_{v}\left(\log \frac{T_{x}}{T_{y}}-\mathrm{I} .408 \log \frac{T_{B}}{T_{y}}\right) \\
& =2.3026 S_{v}\left(\log \frac{P_{x}}{P_{B}}-\mathrm{I} .408 \log \frac{V_{B}}{V_{x}}\right) .
\end{aligned}
$$

When the actual scale of heat-interchanges is desired, the value of the coefficient $2.3026 S_{v}$ must be determined, the mass of the gas involved being carefully considered; or, more simply, the whole calculation may be considered as covering that number of working-strokes which will bring the value of $2.3026 S_{v}$ times the mass of the gases passing through the engine in that period equal to unity. Usually, however, entropy-values to any scale will suffice, in which case the coefficient $2.3026 S_{v}$ may be neglected.

In either of the latter two cases the calculation for any given point, such as $x$, reduces to this simple form :-

$$
\frac{V_{B}}{V_{x}}=\mathrm{r} .490
$$

$$
\log \frac{V_{B}}{V_{x}}=\text { O.I } 732
$$

Adding 0.4 1093

and then 0.008

$\frac{P_{x}}{P_{B}}=\mathrm{I} .778$ $\log \frac{P_{x}}{P_{B}}=\underline{0.2499}$

Subtracting,

$$
N_{x}-N_{B}=-\overline{0.0348}
$$

$\frac{P_{z}}{P_{x}}=3.602$

Adding $\log \frac{P_{z}}{P_{x}}$ to $N_{x}-N_{B}$ above :-

$$
\log \frac{P_{z}}{P_{x}}=\underline{0.5565}
$$

$$
N_{z}-N_{B}=0.5217
$$


Subtracting $\log \frac{V_{B}}{V_{x}}$ from $\log \frac{P_{x}}{P_{B}}, \log \frac{T_{x}}{T_{B}}=0.0767$

Adding,

$$
\log T_{B}=\log 600^{\circ}=2.778 \mathrm{I}
$$

$$
\begin{aligned}
\log T_{x} & =\overline{2.8548} \\
T_{x} & =715.8 \\
t_{x} & =255^{\circ} \mathrm{F} .
\end{aligned}
$$

Adding $\log \frac{P_{z}}{P_{x}}$ to $\log T_{x}$ :

$$
\begin{aligned}
\log T_{z} & =3.41 \mathrm{I} 3 \\
T_{z}^{\prime} & =257^{\circ} \\
t_{z} & =21 \mathrm{I} 7^{\circ} \mathrm{F} .
\end{aligned}
$$

This gives the entropies relatively to $B$ and the absolute temperatures of any desired pair of points. If entropies be desired to true scale, they may now be multiplied by $2.3026 S_{v}$ times the mass of gas passing through the engine per stroke or per unit of time.

The entropy-diagram of the actual cycle thus obtained may then be analyzed in a manner very similar to that given for the steam-engine. In such analysis, as in every portion of the test of which it forms a part, the threefold object of the work should be kept clearly in mind, viz.:-

( I) The determination of the division of the heat supplied in the fuel between the three destinations:-

(a) Absorption by the jacket-water;

(b) Conversion into work;

(c) Rejection in the exhaust;

(2) The comparison of these results with the theoretic possibilities ;

(3) A clear understanding as to the causes of the results observed.

The observations which must be taken in order to complete this programme are:- 
(I) For determination of the heat supplied :

(a) A gas-meter, protected by a gas-bag and properly calibrated;

(b) A gas-calorimeter, preferably a Junkers;

(c) An admission-counter, operated by the admission-valve. ${ }^{1}$

(2) For determination of the conversion of heat into work:

(a) The indicator, equipped with two springs for use as noted below;

(b) A Prony brake or absorption-dynamometer;

(c) A revolution-counter;

(d) An explosion-counter.

(3) For determination of the heat lost to the jacket-water:

(a) The rate of flow, which should be kept constant during and for some time preceding the test;

(b) The temperature at entrance to the jacket;

(c) The temperature at exit from the jacket.

(4) General data:

(a) The barometric pressure;

(b) The temperature of the air entering the suctionpipe;

(c) The temperature of the exhaust-gases (to be attempted only when the engine is running at maximum power, missing no possible explosions);

(d) Chemical analysis of the exhaust-gases, including observation of the presence of uncombined carbon.

(e) The dimensions of the engine, including the volume of the clearance.

1 Where the igniter is suspected of uncertainty of operation this instrument should be supplemented by an explosion-counter. 
As to the taking of indicator-cards, two sets should be secured. One should be with the standard gas-engine indicator, with a spring heavy enough to keep the vibratory waves within proper limit. This will be used as already noted. The other set should be taken with a standard steam-engine indicator, with a spring so light that the compression of the charge brings the indicator-piston to, or quite near to, the top of its stroke. Such diagrams show the phenomena occurring during the exhaust, suction and compression strokes upon an exaggerated scale, with much greater accuracy than the former ones. Those, indeed, should be relied upon for the explosion and expansion curves only.

The diagrams from the light spring will also reveal the negative work involved in overcoming the fluid-friction of admission and exhaust. This should be carefully distinguished from the metallic friction of the engine, in making the report and drawing conclusions. It is usually high in gas-engines, apparently more so than is necessary.

If the spring used for the second set be too strong to let the compression bring the indicator-piston nearly against its upper stop before the explosion, the latter, when it occurs, will wreck the pencil-motion.

As to the explosion-counter, that is most easily rigged by using a second indicator which is always kept open to the engine-cylinder and the drum of which is given a slow continuous or ratchet motion by the engine just sufficient to cover a millimeter or so for each cycle. Its record will consist of a number of saw-teeth, high ones recording explosions and low ones signifying missed explosions. Such a record will check out any failure to ignite charges of gas which the admission-counter has recorded as entering the engine.

If a second indicator be not available, the reliability of the igniter may be tested in this way before and after the regular test. 
The gas-engine test made in this way should result in a report recording : -

(I) The dimensions of the engine and the conditions of the test;

(2) The observations themselves, reduced to averages and totals;

(3) The calculated distribution of the heat to its four destinations, in percentages of the whole;

(4) The four calculated efficiencies, viz.:-

(a) The theoretic or ideal efficiency, calculated from the volume-ratio of compression;

(b) The thermodynamic efficiency, which is the same as the second item of paragraph (3), this page;

(c) The cylinder-efficiency, or (b) divided by $(a)$;

(d) The mechanical efficiency;

(5) Average samples of cycle-diagrams, both from the indicator and in entropy-temperature coördinates ;

(6) The conclusions to be drawn from the foregoing.

(By the time this book has left the press it is probable that the committee of the Am. Soc. M. E. which was appointed to adopt standard methods of making and recording steam-engine and gas-engine tests will have made its final report. This report will be available to most students and should be consulted before undertaking a test.)

\section{Degree of Compression}

In the study of the attainment of maximum actual efficiency in the Otto gas-engine it would seem at first that the problem were a very simple one. The actual efficiency, though less than the theoretic, is very closely proportional to it. The latter is given by the expression

$$
E=\frac{T_{2}-T_{1}}{T_{2}}=\mathrm{I}-\left(\frac{V_{2}}{V_{1}}\right)^{0.402}
$$


wherein the subscripts $\mathrm{I}$ and 2 refer to the conditions at the beginning and end of the compression-stroke respectively. It is obviously easy, in the design of the engine, to make this expression take any value we choose, by variation in the proportion of clearance-volume.

In the development of the engine during the quartercentury which has elapsed since its first appearance, this policy of increasing the efficiency by decreasing the clearance has been steadily pursued. It has constituted the main line of progress up to about I895. Since these thermodynamic principles were well known from the start, it is difficult to see at first why advantage of them was taken so slowly. The explanation is that the mechanical difficulties of construction multiply very rapidly as the degree of compression increases. The maximum pressure incurred at the moment of explosion rises very rapidly with a rise in initial compression. With it must rise the entire cost of construction of the engine; all parts must be strengthened and weighted and bearing-surfaces must be increased. Indeed, so close has constructive practice followed mechanical limitations in this matter that nothing has permitted the later advances into the higher degrees of compression but the steady rise in rotative and piston speeds which has also been a prime characteristic of progress, in gas-engines as in steam-engines. At the higher speeds the force needed to accelerate the reciprocating parts subtracts itself from the fluid-pressure on the piston and protects the bearing-surfaces from momentary overpressure. It is to be noticed at the same time, however, that such inertia-forces fall upon the bearing-surfaces unmodified by fluid-forces during the suction and exhaust strokes; they may not be increased recklessly, therefore.

Another limit to the feasible degree of compression is found in the danger of pre-igniting the charge by the heat 
of compression. This limit appears earlier in oil-engines than in gas-engines. It is a phenomenon which may be taken advantage of, however. All engines relying upon hot-tube ignition without the aid of timing-valves depend upon it, more or less unconsciously, for ignition. The Diesel engine relies upon it absolutely and completely; adopting a maximum pressure of compression of from 500 to 550 pounds per square inch, it insures at dead-centre an atmosphere within the cylinder all portions of which are above the temperature of ignition. Ignition is timed in this case by the preservation of the fuel separate from the air until the beginning of the working-stroke. It is then fed into the hot compressed air under control and ignites because of the high temperature of the air. The most valuable result yet derived from the Diesel engine is the demonstration that in such an atmosphere as this any sort of liquid fuel, whether light or heavy, crude or pure, may be injected without preliminary vaporization and the resultant combustion will be complete. ${ }^{1}$ Referring again to the analysis of the theory of the Diesel cycle, which was presented on page I49, it is to be noted that its high efficiency is due primarily to its high degree of compression rather than to any peculiarities of form of cycle, method of ignition, "isothermal combustion," etc.

Complete Expansion. - The next line of effort at improvement of efficiency, taken in the chronological order of their appearance, was the avoidance of incomplete expansion and incomplete exhaust of the burned gases. These features, it will be remembered, are inseparable from an Ottocycle engine in which all of the processes are enacted in a single cylinder. They are now accepted as such, but only after much time and money and some of the best skill in

1 “Mittheilungen über den Dieselschen, Wärmemotor," by Rudolf Diesel. Zeitschrift d. Vereines Deutsche Ingenieure, Vol. XXXXIII. 
the land has been expended in proving the impracticability of their avoidance. Chief amongst the workers in this line was Mr. Atkinson, who made two full attempts at designing an engine which should completely expand and exhaust its burnt gases. In the first a horizontal cylinder open at both ends contained two trunk-pistons placed head to head. Each of these was connected to the engine-crank by a peculiar arrangement of links and walking-beams whereby each piston had a peculiar and independent motion of its own. Considering first the relative motion between the two pistons, it may be described as producing the following sequence of events :-

(I) The close juxtaposition of the two, which constituted the beginning of the suction-stroke;

(2) Their separation by a certain distance, whereby the volume between them was filled with an explosive charge at atmospheric pressure;

(3) Their approach by a lesser distance, whereby compression was performed, at the end of which process ignition took place;

(4) Their separation by a distance greater than (2), whereby expansion took place much more completely than in the ordinary engine;

(5) Their close approach, into position (I), whereby complete exhaust was effected.

This engine was known as Atkinson's "Differential" engine. Not proving a mechanical success, it was followed by his so-called "Cycle" engine, in which the same series of changes of volume was produced in a cylinder closed at one end and containing a single trunk-piston, by a peculiar radial gear between piston and crank. Both engines gave good thermodynamic results. Both were complete failures mechanically. The work was done so 
well, however, that the failures are now regarded as inevitable in that line of effort.

Scavenging. - Although the completion of expansion in the Otto-type engine still remains an unsolved problem, the completion of exhaust has been accomplished in a simpler way. For a time a number of designers advocated the insertion between each two-revolution cycle of one complete idle revolution, the end in view being the pumping of a fresh charge of air through the engine, whereby the bulk of the burnt gases remaining in the clearancevolume should be swept out. But the gain was not found to be worth the mechanical and commercial disadvantages incurred, and the plan is now no longer followed. Later Mr. Atkinson evolved the beautifully simple plan of producing a partial vacuum in the cylinder during the exhauststroke by making the exhaust-pipe some sixty feet long or more, and quite straight and free. The inertia of this long column of gases, when once set into rapid motion by the first sharp puff of the exhaust, would draw into and through the cylinder quite a draft of pure air, washing out the bulk of the burnt gases in the clearance. The thermodynamic effect of this plan was excellent. His early experiments with it reported a saving of some $5 \%$, a quite profitable one considering the ease with which it is attained.

Scavenging, in some form or other, is now considered an essential feature of all large explosive engines attempting refined efficiency. In the latest types of heavy engines for use with blast-furnace gases it is performed by a small separate blowing-cylinder. ${ }^{1}$

Regulation. - Of all the problems connected with the design of the Otto-type of gas-engine, however, none has

1 See Humphrey's paper on “ Recent Progress in Large Gas-engines," read before the British Association at Belfast, Sept. II, 1902. Also abstracted in London Engineering, Sept. 19 et seq. 
a more urgent bearing upon the thermodynamics of the efficiency-problem than that of regulation. It will be remembered that all of the early designs operated upon what is called, most properly, the "hit-or-miss" plan of regulation. That is, so long as the engine were below normal speed there was no hindrance to, nor modification of, the development of full maximum power. When the speed rose above normal, all development of power was shut off completely by the omission of gas-supply on one or more of the suction-strokes. There was no gradation between these two extremes.

Many of the smaller engines, and a few of the larger ones, still adhere to this plan. When it is remembered that the single-cylinder gas-engine has three idle strokes to one working stroke, at the best, and that the dropping out of one working stroke leaves seven idle strokes before another impulse can be received; when it is further remembered that this seven-to-one ratio applies to a reduction of power to only half the maximum, or about seven-tenths the proper rating, and that for further reductions the proportion of idle strokes to impulses may be increased to eleven, fifteen, or nineteen to one; when it is finally remembered that the power needed for compressing the next charge to be fired must be taken out of the fly-wheels at just the moment when they are the weakest and after they have already fallen below normal speed; when all of these points are compared with modern demands and attainments in steam-engine regulation, the hit-or-miss plan of regulating gas-engines must appear as a crudity only comparable with steam-engine practice of a century ago.

Nor can any remedy possibly be hoped for in the governor. The above remarks would apply with complete force were the governor ideally perfect. It is true that in many small gas-engines the governor is abnormally and 
inexcusably bad, and that the speed-variations are thereby much exaggerated over what is inevitable. In fact, no gasengine operating upon this plan is fitted with a good governor. The governor is always one of two types, both of which are intrinsically unsuited to the work, viz.: (I) A very small and weak centrifugal governor, dimensioned so as to have great stability; (2) an inertia-governor, usually of the pendulum-type.

The latter is unsuited because its action depends upon acceleration; it can do nothing if not to keep the engine's acceleration, positive or negative, within certain limits. But the Otto-type of gas-engine necessarily involves a degree of acceleration within each cycle, positive during the expansion-stroke and negative during the compressionstroke, which far exceeds any degree of general acceleration in average speed which is permissible in good regulation.

The former type is unsuited because the range of speedvariation which is involved in stability and which constitutes a valuable factor for control in steam-engine governors is here entirely unavailing. The governor can do nothing but one extreme thing or the other, either to admit maximum power or cut off all of it, and a field of gradation between is powerless to accomplish gradation. Moreover, owing to these extremes in distribution of power, positive acceleration will be prompt and powerful; in addition, from one to four strokes must elapse before action on the part of the governor can become effective. The need is, therefore, for a governor which will signalize departure from normal speed with the utmost promptness and emphasis. Such is the perfectly isochronous pendulum governor, a type entirely unfit for steam-engine regulation. It is the only one fit for the hit-or-miss plan of controlling gas-engine speeds. It has never, so far as the writer's knowledge extends, been applied. 
It is no object of the present text to properly discuss questions of regulation other than as they affect thermodynamic problems. But the latter cannot be correctly entered upon without previous acceptance of the premises that the hit-or-miss plan of governing is intolerable, according to all steam-engine standards of excellence, and must inevitably remain so. This being true, what other plans are available? Two only, viz. :-

(I) Reduction of amount of gas present at the moment of ignition.

(2) Reduction of the pressure prevailing at the moment of ignition, by the reduction of either the volume (by cut-off) or the pressure (by throttling) of the charge drawn in during suction.

Either (I) or (2) permits the gradual reduction of the vigor of each impulse, without diminishing their number, until only a small fraction of normal power is developed. Either plan inevitably involves some loss of efficiency from the maximum. In the case of the first plan this is due to the inevitably slower combustion in weak mixtures, and is so bad as to be intolerable. Reference to the theory of the Otto cycle will confirm the statement as to the second plan also. In the expression for its theoretic efficiency, $\frac{T_{2}-T_{1}}{T_{2}}$, reduction of the degree of compression reduces the maximum efficiency attainable. With the second plan it is only a partial, but a valuable, compensation that decrease of the degree of compression (in a fixed clearancevolume) increases the ratio of expansion inito that territory which was stated, on page 148 , Part I, as belonging exclusively to the Lenoir cycle.

The objections to both plans can be modified by skilful design and particularly by a wise combination of the two. 
Practically all of the larger and more important engines have accepted the premises stated above as to the hit-ormiss plan and rely upon graded impulses for regulation.

It is further to be remarked that variation of the power developed, with or without variation of the degree of compression prevailing at the moment of ignition, is obtainable by any one of three methods, viz.:-

(I) Gradation of pressure of charge during suction, or by throttling.

(2) Gradation of volume of atmospheric charge during suction, or by variable cut-off.

(3) Gradation of final volume of compression, or by variable clearance-volume.

The first two are very nearly alike; their difference is visible in Fig. 56. The second has the advantage over the first by a narrow margin of efficiency and the disadvantage of greater mechanical complexity. The third, so far as the writer is acquainted, has never been attempted.

To illustrate these points is published herewith a set of indicator-cards, with entropy-analyses thereof, from an $\mathrm{II}^{\prime \prime} \times \mathrm{I}^{\prime \prime}$ two-cylinder gas-engine, running at 290 revolutions per minute. The conditions under which the cards were taken were in every way normal and were graded from an overload of $17.4 \%$ above rating down to the friction of the engine alone. The fuel-consumption near rated load varies between Io and Io.6 cubic feet of natural gas per brake horse-power, the gas having an average calorific power of Io00 B.t.u. per cubic foot. This represents a thermodynamic efficiency of from 24 to $25.5 \%$. The variation of the fuel-rate with the load is remarkably constant. Calling the efficiency at rated load IOO, the indicator-cards show it as rising to about II2 under the overload and falling to about 75 at half-load. 
This is accomplished, so far as the cards reveal the method, by relying upon more or less throttling of the incoming charge under all conditions of load, even the maximum, and by thereby securing an expansion to a volume greater than the initial when the latter is measured at atmospheric pressure. The percentage-fraction of the stroke at which the charge is at atmospheric pressure is shown by the figures beneath the atmospheric line. It will be seen that only 7I\% of a cylinderful of fresh charge is taken in even under maximum load. At normal

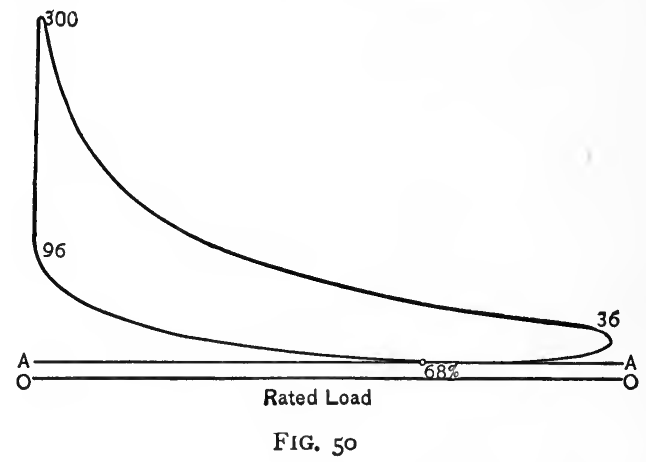

load it is only $68 \%$. At the same time, the clearance is made less than usual (only about $21.5 \%$ ) so that the proper degree of compression is retained. The reduced pressure during suction, due to the throttling by the governor, and the work lost thereby, could be shown (without confusion of lines) only in Fig. 56, and there only approximately.

While the governor is reducing the power by throttling the charge, it is also reducing the proportion of gas present, making the inflammation slower, which is quite permissible in view of the excessive opportunity for expansion present during the lighter loads.

As these diagrams are typical representatives of the best 
practice of the day with large engines, it is proper to use them as a text for a discussion of the possibilities for further progress, as revealed by thermodynamic considerations.

In the first place, the figures given above for the variation of efficiency in terms of variation of load are misleading, in that the efficiency at rated load was taken as the

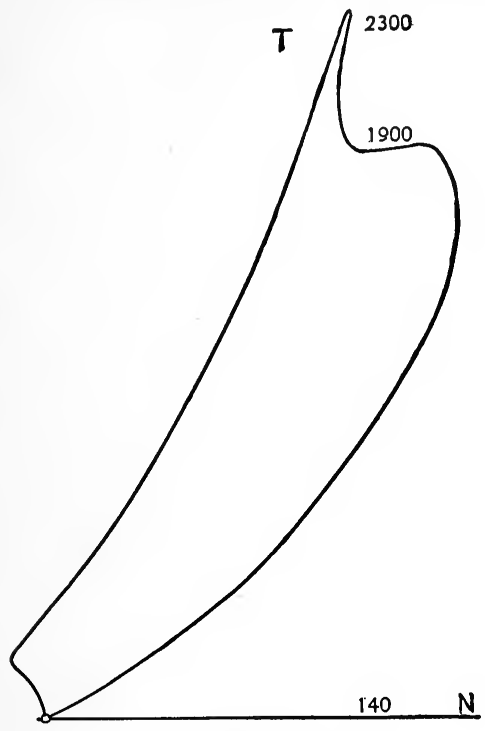

Rated Load.

FIG. 5I

standard. It is one of the marked distinctions between steam-engine and gas-engine practice that, whereas in steam-engines (except in the almost obsolete throttlegoverned type) the maximum efficiency is found at or slightly below rated load, decreasing as the load either increases above or falls to a fraction of the rating, in gasengines the efficiency is always the greatest when the load is the greatest. This fact led to the very unfortunate practice, which is only now just dying out, of rating gas- 
engines so high that they could seldom be driven appreciably above their rating, and often not fully up to it. Even the much more liberal practice shown in the accompanying diagrams reveals a maximum load only $18 \%$ above the rating; and, while the writer does not know that this is the extreme maximum capacity of the engine, it is plain, from the degree of compression incurred at this power, that it could not possibly rise much higher. This margin above rating has been obtained, too, by artificially adopting,

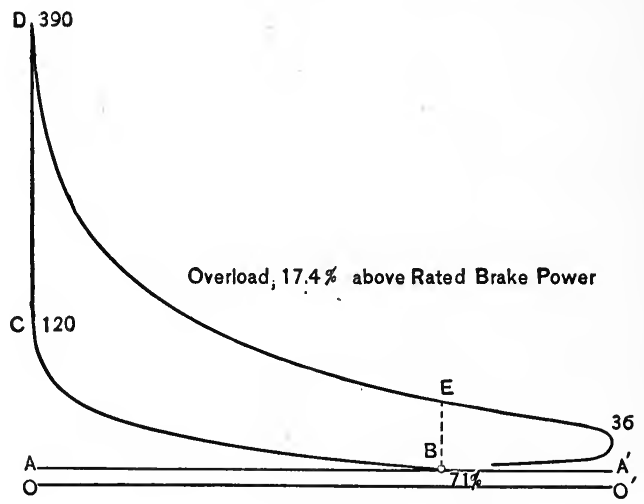

FIG. 52

as the standard efficiency of the engine, an efficiency which is only some $78 \%$ of what the engine plainly shows that it can do when given a chance. Yet, after all, the margin of power above the rating, $18 \%$, which is purchased at the price of the loss of $22 \%$ of the efficiency, is very small when compared with standard steam-engine practice, and plainly reveals one of the chief reasons why the gas-engine fails to meet more universally the demands of the industrial world for a prime mover. It does not possess sufficient margin of power. (See curve $W_{B}$, Fig. 58.)

Does theory offer any promise of remedy for this situa- 
tion? It does, very plainly. The remedy may not be easily attainable in constructive practice, but it is plainly there. In the writer's opinion, moreover, the reduction of it to practice does not involve any obstacles impossible of conquest.

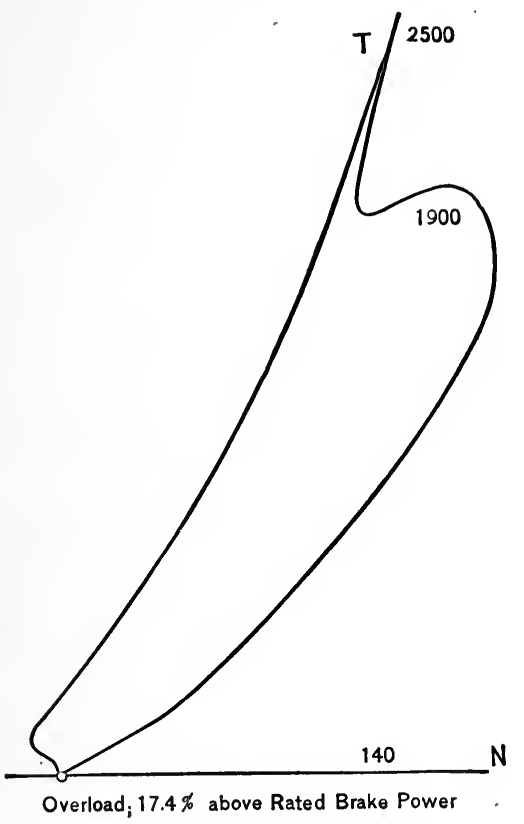

FIG. 53

The practice, as well as the theory, of the governing of Otto-cycle engines by throttling or cutting off the incoming charge shows that the maximum efficiency is obtained when the least throttling is done and the cycle approaches most nearly to the unmodified Beau-de-Rochas cycle. The departure from the latter is undertaken, very plainly, for the sake of margin of power and for better regulation, which are purely mechanical questions, and quite in the face of all considerations of efficiency. 
The efficiency of the Beau-de-Rochas cycle is given by the expression $\frac{T_{2}-T_{1}}{T_{2}}$, wherein the $T$ 's refer to temperatures before and after compression. Therefore, very plainly, the degree of compression should always be kept constant, at the maximum point permissible from mechanical considerations of bearing-surfaces and strength of parts or from danger of pre-ignition. At the 'same time, since any reduction of the proportion of gas in the fresh charge leads to a slower rate of combustion, which is fatal to efficiency, that should be kept constant too. Since any reduction of the pressure of the fresh

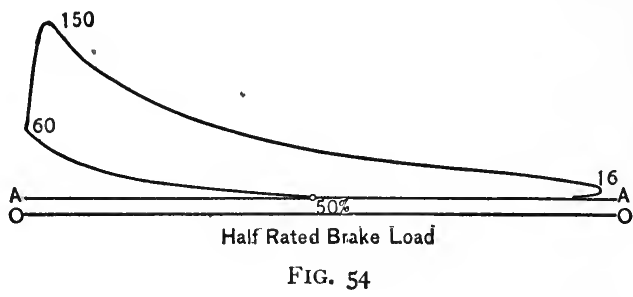

charge below atmospheric leads to loss of power in recompression, not to mention the much greater loss due to the diminished pressure at the instant of ignition which has been discussed above, that, too, should be avoided; yet it is the least objectionable in the list.

If all of these rules are to be observed, while the power developed and the gas taken in are reduced to suit a diminished resistance, only the following policy is left open to the designer:-

(I) To reduce the volume of the atmospheric charge of standard mixture taken in ;

(2) To reduce the clearance-volume in like proportion, in order to maintain a constant degree of compression. 
The first cannot be accomplished, in the standard Otto engine, except by recourse to an automatic cut-off. As the throttling of the charge is a method only slightly inferior to this thermodynamically, and superior to it mechanically, this first point will be disposed of by leaving these alternatives open to the designer without further discussion.

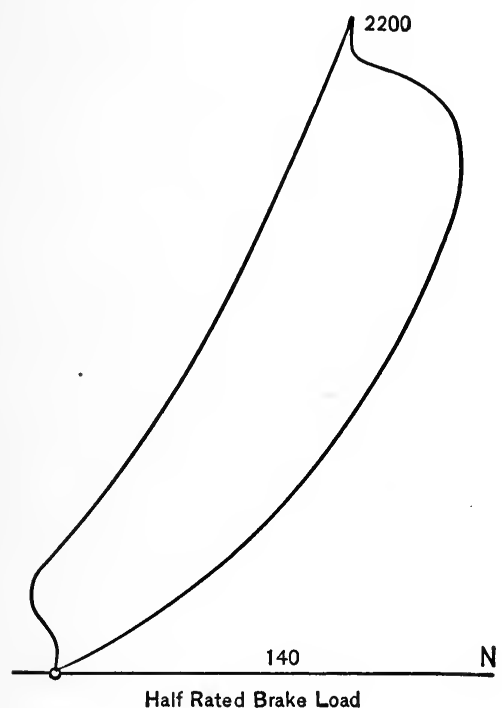

FiG. 55

(It should be said, however, that the friction in getting the gases into and out of a gas-engine cylinder usually constitutes as much as two-thirds of its entire frictional loss, and this is much exaggerated where throttling is substituted for automatic cut-off. The entire question of enginefriction is one of the most unsatisfactory ones connected with gas-engine design. In the present case the mechanical efficiency is between 75 and $80 \%$, which is certainly much below what engineering skill ought to be able to com- 
mand. The reasons for the present faulty situation are two: (I) Combustion within the cylinder, demanding small poppet-valves for admission and exhaust; (2) the double transmission of the energy required for compression, first from the piston through all the oblique connections and bearings to the fly-wheel and afterward back again. Neither of these may be remedied without radical departure from existing arrangement of parts.)

As to the second rule essential to efficiency, the variation of clearance-volume, as this is not a book upon constructive mechanics, the question as to how variation of the clearance-volume is to be accomplished will not be opened.

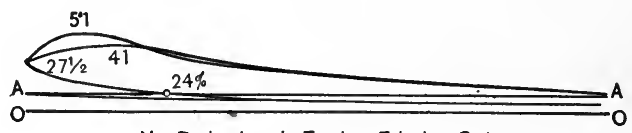

No Brake Load: Engine=Friction Only.

FIG. 56

It is quite proper, however, to inquire as to what would be the thermodynamic results were such a construction operatively accomplished.

For this purpose let Fig. 52 be taken as representing the best of modern practice in the handling of gas-engine fluids in an engine-cylinder. Let the new engine be arranged to develop, under maximum load, the facsimile of Fig. 52 chopped off at $B E$, or the indicator-diagram $B C D E$. This would call for a clearance of about 30\%. But this $30 \%$ must be supposed to be the maximum limit of a clearance which is variable by the governor from $30 \%$ down to the minimum practicable.

If, now, for all lesser loads than maximum the initial volume of the charge $A B$ be diminished in somewhat equal proportion, and the clearance-volume be diminished in exact 
proportion with $A B$, the indicator-cards developed would be the exact likeness of $B C D E$, Fig. 52, upon a diminished longitudinal scale, and would develop a like efficiency; except that now the terminal pressure $E B$ has opportunity for further expansion during the remainder of the stroke, to the right of the new positions of $E$. This added expansion is not obtained, as is the case in all existing engines, at the expense of diminished efficiency of the portion $B C D E$, due to diminished compression. It is all pure gain, and serves

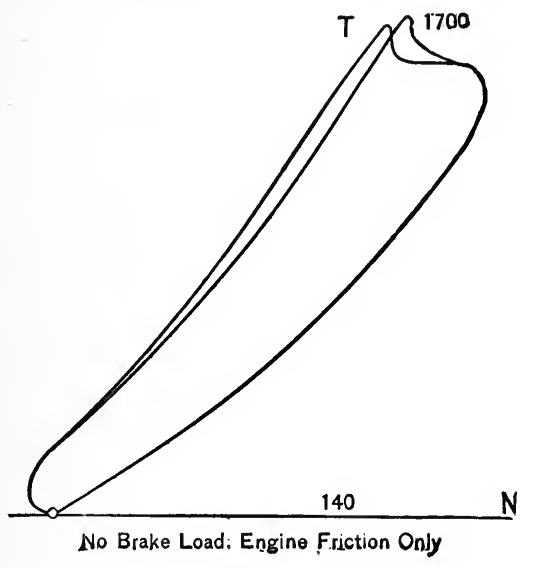

FIG. 57

to make the efficiency an increasing instead of a decreasing function of the load as the latter falls from maximum.

The movement of $B$ toward $A$ under the influence of the governor as the load diminishes would result in the variation of efficiency with load according to the curves of Fig. 58. In Fig. 58 the actual diagram $B C D E$ of Fig. 52 is taken in each case as a standard for comparison of 100. The curve $W_{B}$ shows the approximate relation of the brakeefficiency of the existing gas-engine under discussion to its brake-load. The curve $N_{B}$ shows the same relation for 
the proposed new engine with variable clearance-volume controlled by the governor. The curve $N_{I}$ shows the relation of indicated efficiency to indicated power for the new engine.

In considering these curves, it must be remembered that the curve $N_{B}$ is a theoretic one only in the supposition of altered dimensions of clearance-space. All the inevitable departures of actual results from theoretic possibilities are

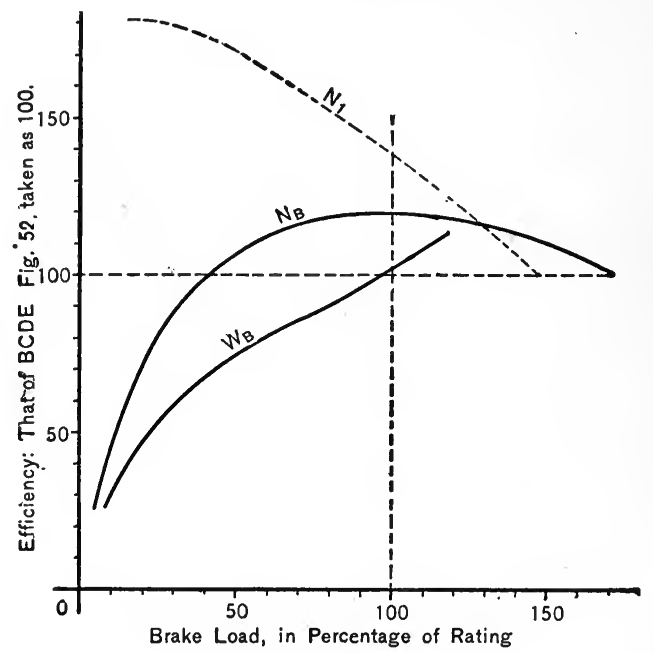

FIG. $5^{8}$

included in the premises. It is also to be noted that the curve $W_{B}$ represents, not poor engineering practice which needs rebuke, but what is possibly the best, and surely near to the best, of modern practice. The curve $N_{B}$ shows in comparison the undeveloped possibilities of the Otto engine, so far as the writer yet perceives them.

In comparing the curves $W_{B}$ and $N_{B}$, the point of probably greater importance than the generally higher average efficiency of the latter is the wide range of its capacity. 
The rating as chosen shows a surplus margin of capacity of $70 \%$. It is probable that the average builder would prefer to rate such an engine at about $\mathrm{I} 20$ (on the arbitrary scale adopted), reducing its margin of surplus of maximum above rated power to some $4 \mathrm{I} \%$, but at the same time reducing the lower limit of fractional load which might be imposed without perceptible detriment to the efficiency to $50 \%$. The limit to reduction of power without passing below what is now first-class gas-engine efficiency at rated load would be thereby reduced to $33 \%$. In other words, an engine consisting of a pair of $\mathrm{I}^{\prime \prime} \times \mathrm{I}_{4}$ " single-acting cylinders running at 275 r.p.m., if equipped with governorcontrolled variable clearance, would rate at 60 horse-power and would develop that power at a fuel-rate of $8 \frac{1}{4}$ to 9 cubic feet of natural gas per brake horse-power. It would develop a maximum brake horse-power of 85 without losing more than $17 \%$ of its best efficiency, and could develop as little as 30 horse-power without falling below an efficiency now attained only at the maximum capacity of the actual engine adopted as a basis for comparison. It could develop as little as 20 horse-power without falling below the fuel-rate which it demanded at its maximum capacity, and which is the same as that of the best existing engines under rated load.

The curve $N_{B}$ is of the same general form as that which has always been revealed by the standard steam-engine. It expresses characteristics which are essential to the broad success of any prime-mover. Their incorporation into the explosive gas-engine would constitute a long stride forward toward that mechanical parallelism with the steam-engine which alone can mark the completion of its growth.

Before leaving the set of diagrams which have formed the text of the foregoing discussion, attention should be 
given to the entropy-diagrams, Figs. $5 \mathrm{I}, 53,55$, and 57 . There lies in them much food for instructive thought. The only point to which particular notice is to be called at present is the persistent concavity (toward the right) of the expansion-lines, in spite of the greatest variety of form, from sharp peak to flatly rounded top, on the part of the pressure-volume curve. This, and the approach of the later stages of expansion to isothermal conditions, hold true of practically every card the writer has ever analyzed. They leave no room for doubt as to the entire completion of combustion almost immediately after ignition and the sole activity of cylinder-wall influence in the deformation of the expansion-line from a true adiabatic. They also prove beyond question that the indicator-card alone, untranslated into the entropy-temperature diagram, throws about as brilliant a light upon the real nature of the processes transpiring within the cylinder as would a German work upon thermodynamics to one who knew no German.

The isothermal character of the expansion in its later stages would go to show that the gain in efficiency due to prolonging it by means of variable clearance might easily be greater than that calculated above. 
APPENDIX 



\title{
APPENDIX
}

\section{TABLE OF SPECIFIC AND LATENT HEATS OF VARIOUS SUBSTANCES}

\author{
SPECIFIC HEATS
}

\section{SolidS}

\begin{tabular}{|c|c|c|c|c|c|c|c|}
\hline Aluminum & . & . $0.2 \mathrm{I}$ & Iron, at $3^{2^{\circ}}$ & - 0.110 & Silver. & • & . 0.057 \\
\hline Brass . & - & - 0.092 & Iron, at $600^{\circ}$ & - 0.130 & Clay . & • & . 0.055 \\
\hline Copper, at & $32^{\circ}$ & . 0.090 & Tin . & . 0.055 & Stone. & . & . 0.20 \\
\hline Copper, at & $600^{\circ}$ & 0.099 & Zinc. & . 0.095 & Glass . & . & - $\quad 0.18$ \\
\hline Mercury & . & . 0.033 & Lead. & - 0.030 & Ice . & . & . 0.50 \\
\hline
\end{tabular}

\section{LIQUIDS}

Anhydrous ammonia Bisulphide of carbon Ether
Alcohol • • • . . $\quad 0.6$ Oils . $\quad . \quad . \quad . \quad 0.4$ to 0.5

\section{GASES}

\begin{tabular}{|c|c|c|c|c|c|c|}
\hline & & & $\begin{array}{c}\text { Under } \\
\text { Const. Press. }\end{array}$ & $\begin{array}{c}\text { Under } \\
\text { Const. Vol. }\end{array}$ & Ratio & Density \\
\hline \multirow{3}{*}{\multicolumn{2}{|c|}{$\begin{array}{l}\text { Atmospheric air } \\
\text { Ammonia (anhydrous) } \\
\text { Bisulphide of carbon }\end{array}$}} & . & 0.237 & 0.169 & 1.4024 & $1.000^{\circ}$ \\
\hline & & - & $0.5^{2}$ & $0.4 \mathrm{I}$ & 1.27 & 0.59 \\
\hline & & & 0.16 & $\longrightarrow$ & - & 1.266 \\
\hline Carbonic acid & - & • & 0.217 & 0.167 & 1.30 & 1.520 \\
\hline Carbonic oxide & - & • & 0.242 & 0.173 & 1.40 & 0.967 \\
\hline Ether . - & - & - & 0.47 & 0.45 & 1.04 & 2.557 \\
\hline Hydrogen, at $0^{\circ}$ & . & - & 3.40 & 2.41 & I.4I & 0.069 \\
\hline Hydrogen, at 100 & & ${ }^{\circ}$ & 3.41 & 2.42 & $\mathrm{I} .4 \mathrm{I}$ & - \\
\hline Methane & - & - & 0.593 & $0.45^{\circ}$ & 1.316 & 0.555 \\
\hline Nitrogen & - & - & 0.244 & 0.173 & 1.41 & $0.97 \mathrm{I}$ \\
\hline Oxygen . & . & - & 0.217 & 0.155 & $\mathrm{x} .40$ & x.106 \\
\hline Superheated stean & & - & 0.6 & 0.46 & 1.3 & 0.622 \\
\hline Alcohol . & - & - & 0.453 & 0.398 & 1.14 & $\mathbf{x} .5^{89}$ \\
\hline
\end{tabular}




\section{LATENT HEATS}

\section{Of the Melting of Solids}

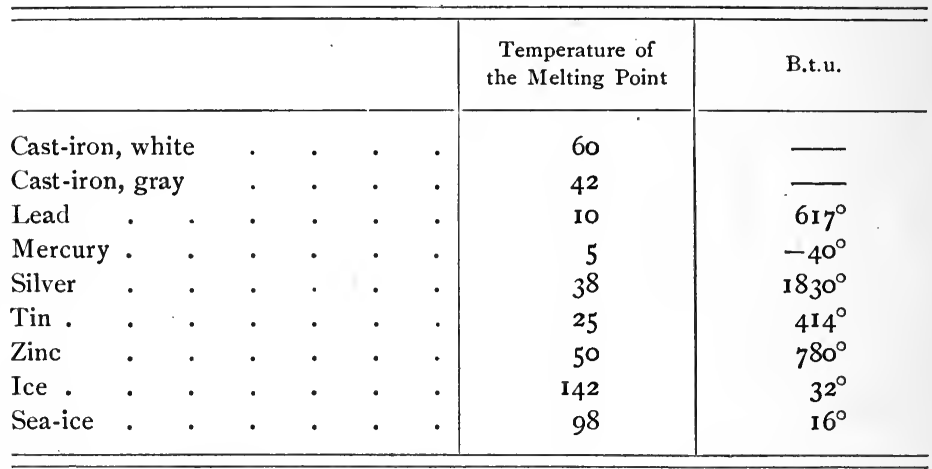

OF THE VAPORIZATION OF LiQUIDS

Ammonia (anhydrous)

Bisulphide of carbon .

Carbonic acid

Ether .

For the above values the author is chiefly indebted to the authorities cited in Landolt \& Börustein. He has also drawn upon the table in Pupin's "Thermodynamics."

\section{THE VARIABILITY OF THE SPECIFIC HEATS}

In the preceding work it was invariably assumed, except in the case of water, that the specific heats of all substances were constant for all temperatures. In the thermodynamics of superheated steam and of the gas- and oil-engines the truth or falsity of this assumption becomes important. It would not, however, affect the graphical methods or the general conclusions reached in the discussions presented, and for this reason its brief treatment has been reserved to this point, as a refinement which might be added by those so inclined. 
Several scientific investigators, including Regnault, have maintained the constancy of the specific heats under varying pressure and temperature. Nevertheless, the weight of evidence is overwhelmingly to the opposite. But the variations appear to be peculiar to each gas investigated, and no general laws have been established. It generally appears that the specific heats increase with both pressure and temperature, at least for all gases of interest to the engineer. The maximum is reached at a pressure of from 350 to 400 atmospheres; with temperatures it seems to rise indefinitely. A few experimental results are given, to indicate the state of the science.

The Permanent Gases. - As these become of interest chiefly in connection with gas-engine problems, the reader is referred to the able work done by Professor Burstall for the Gas-engine Research Committee of the Brit. Inst. M. E. Professor Burstall quotes the results given by Mallard and Le Chatelier for $S_{V}$ as : -
Oxygen, . $\quad 0.1488+0.0000763 t$;
Nitrogen, . $0.170+0.0000872 t$;
Carbonic acid, . $0.1477+0.000176 t$;
Superheated steam, $0.32 \mathrm{I}+0.000219 t$;

wherein $t$ is temperature Fahrenheit.

Professor Burstall adds the conclusions which he reached as to the variability of the specific heat of gas-engine mixtures in terms of the richness of mixture. The writer has taken the liberty of presenting them, for convenience, in the form of Fig. 59. It gives the values (on the vertical scale) of $A$ and $B$ in the formula -

$$
S_{p}=0.2400+\frac{A}{10,000}+\frac{B}{\mathrm{I}, 000,000},
$$

in terms of the proportion of air to gas in the mixture by volume. (See also Batelli, in Rapports Présentés au Congres International de Physique, 190o, and to the authorities referred to therein.)

Superheated Steam. - Aside from the value of Mallard and Le Chatelier given above only one set of results is given for super- 


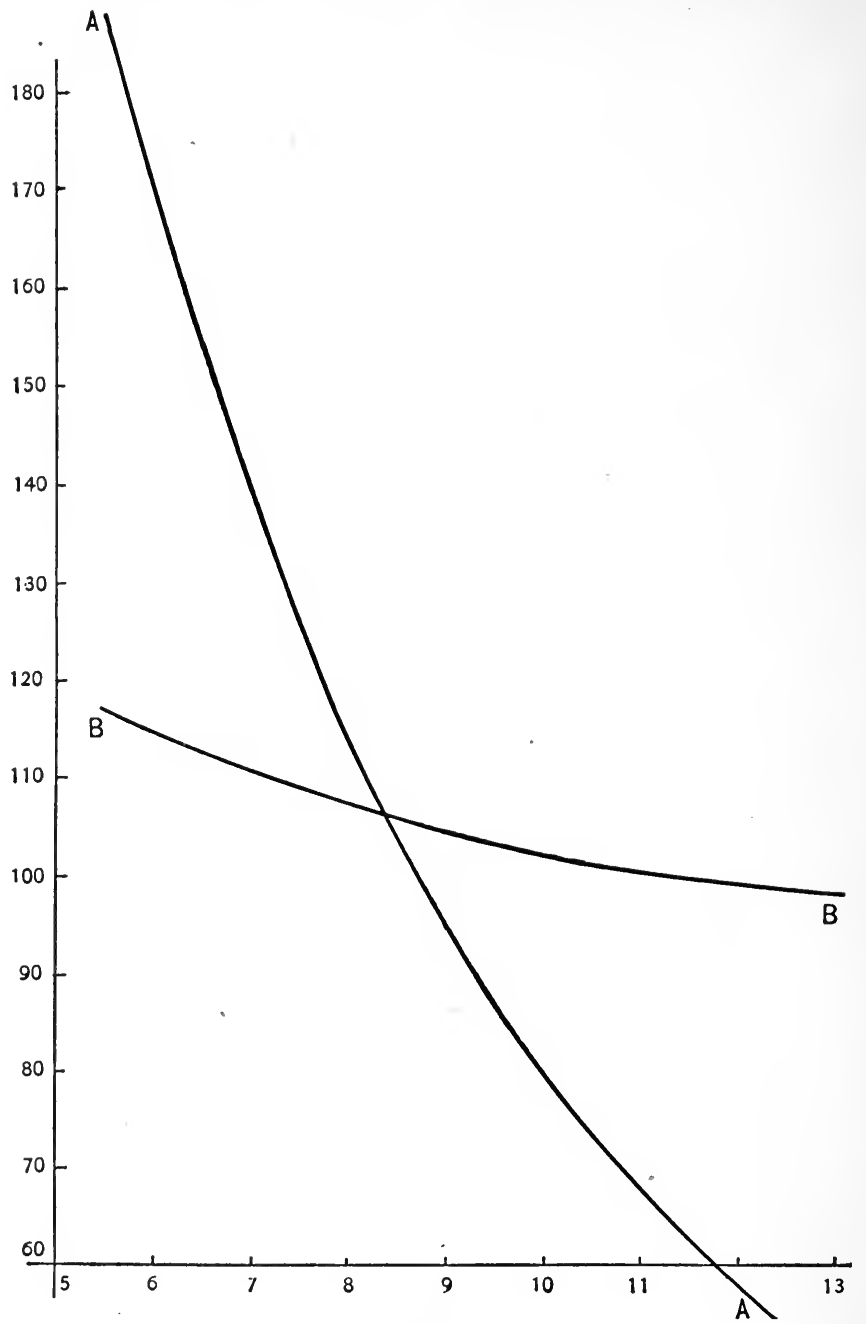

FIG. 59

Proportion of Air to Gas, by Volume. 
heated steam, that reported by Grindley (Phil. Trans., Vol. 194). These experiments are very carefully made ; they are quite recent ; they should be significant. 'The investigator reports, as his general conclusions, that the specific heat under constant pressure is independent of the pressure, but varies with the temperature. His conclusions, and also his tabulation of his calculations from Hirn's experiments, are appended.

VALUES OF THE MEAN SPECIFIC HEAT, UNDER CONSTANT PRESSURE, OF SUPERHEATED STEAM AT ATMOSPHERIC TEMPERATURE

\section{HIRN}

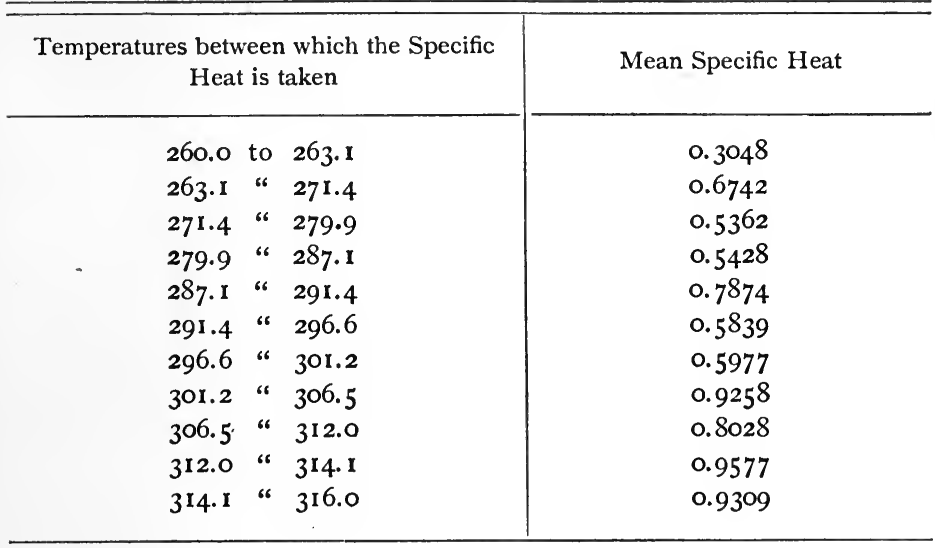

GRINDLEY

Temperatures between which the Specific Heat is taken
Mean Specific Heat
0.4317
0.4778
0.5152
0.5646
0.6482 
Because the above results do not seem to be altogether consistent, the writer has taken the liberty of recalculating the values which seem to him to be pointed to by the data which Grindley records as the result of his investigation, and without reducing them to atmospheric pressure. These values are displayed in Fig. 6o, which shows that portion of the entropy-temperature, or, to give it its proper name, the thermal, diagram covering the field investigated. $S S$ is the saturation-curve. PPPP are constant-pressure lines, their level being noted where they cross $S S$. $Q Q Q Q$ are the constant-heat curves upon which Grindley conducted his inquiry. The temperature-scale is in degrees Fahrenheit.

Had Grindley chosen a fixed set of lower pressures to which to wire-draw the steam, the field would have been checkered by these $P$ and $Q$ lines, between each pair of intersections of which would have been known the specific heat with great accuracy, averaged over a comparatively slight distance, viz., that between the lines. As it is, the results given are located in no regular distribution over the field. They are most of them, too, not only averages over quite extended temperature-ranges, but over ranges which lap one over the other. Nevertheless, enough is shown definitely to prove conclusively : -

(I) That the specific heat is not independent of pressure, but varies directly with it.

(2) That it varies directly with the temperature.

(3) That it varies inversely with the entropy. Lines of constant specific heat cannot be located definitely; but it is clear that they would have a generally northeasterly trend.

Whether law (3) above, taken with the hypothesis that entropy is a close function of molecular mass, has any connection with the relation between specific heat and molecular weight is a question for the physicists to answer.

In view of the important rôle which superheated steam is beginning to play in the steam-engineering industry, the need for further investigation is obvious. 


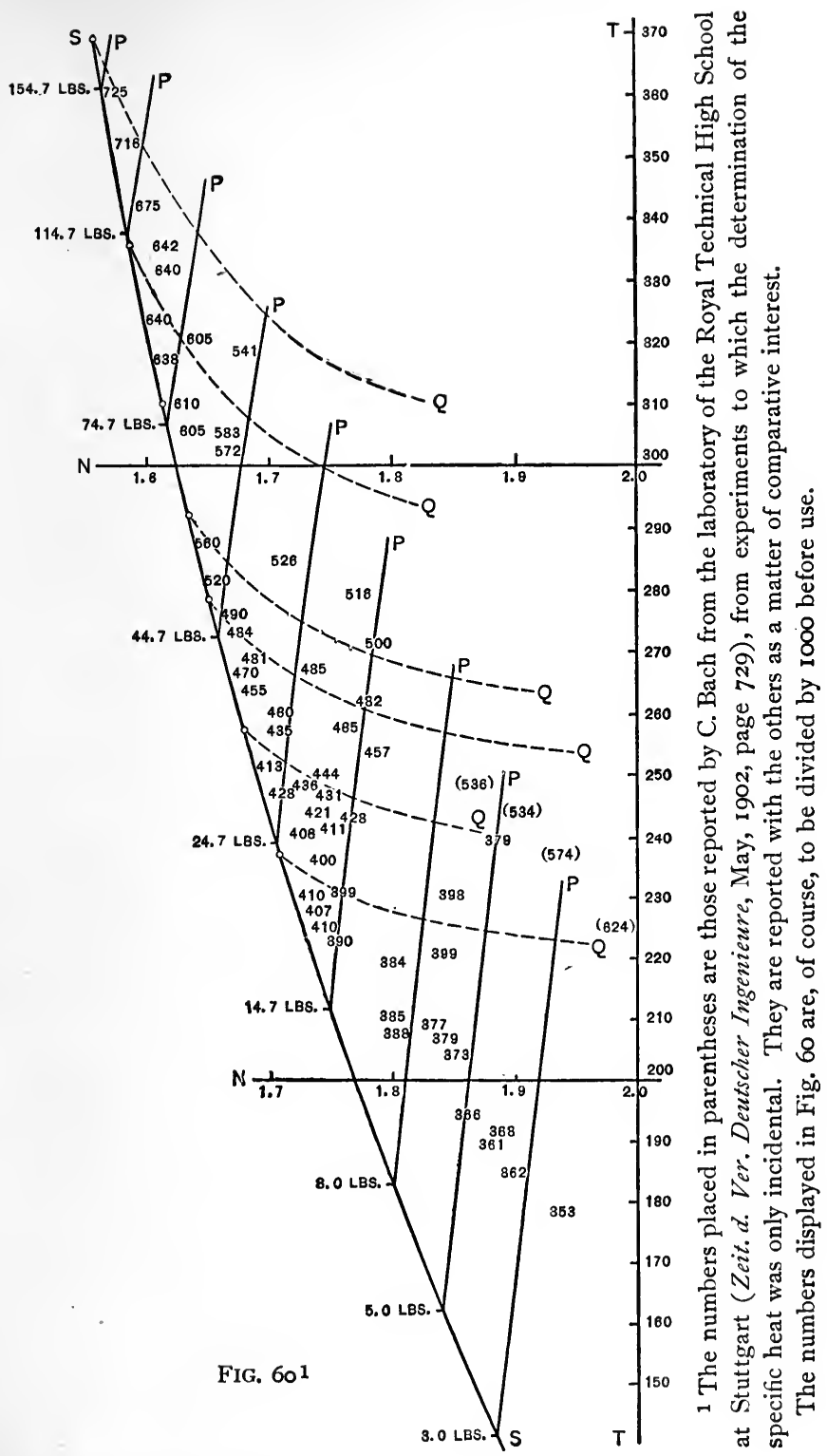




\section{A TABLE OF NATURAL LOGARITHMS}

Note. - The student is reminded that in using natural logarithms the logarithm of a number ten or one hundred times as great as any number contained in the table cannot be gotten, as in common logarithms, by adding one or two integers, which are the common logarithms of 10 and 100, to the tabulated logarithm. Instead, to the tabulated logarithm must be added one or two or more times the natural logarithm of Io, which is 2.302585 .

\begin{tabular}{|c|c|c|c|c|c|c|c|c|c|c|}
\hline & O & 1 & 2 & 3 & 4 & 5 & 6 & 7 & 8 & 9 \\
\hline 1.00 & 0.00 & 0.00100 & 0.00200 & 0.00300 & 0.00399 & 0.00499 & 0.00598 & 0.00698 & 0.00797 & 0.00896 \\
\hline 1. & 0.00 & 94 & 0 & 0 & 0 & 89 & $0.015^{8} 7$ & 0.01686 & & 82 \\
\hline & & & & & 0.02372 & & 67 & & & \\
\hline & 0.02 & 0.03053 & 0.03150 & 0.03247 & 0.03344 & 0.0 & 0.03537 & & & \\
\hline 1.0 & & I 8 & 0.04 & 0.0 & 0.04306 & & 0.04497 & & & 784 \\
\hline & & & & & 0.05259 & & 0.05449 & & & \\
\hline & & & & & & & & & & \\
\hline 1.0 & & & o & 0.0 & & & 25 & & I I & 603 \\
\hline & & & & & & & & & & \\
\hline & & & & & & & 0.09167 & & & \\
\hline 1.10 & 0.09 & 0.09 & 0.09713 & 0. & 94 & 85 & 0.10075 & 0.10165 & 256 & .10346 \\
\hline 1.1 & $0.0953 \mathrm{I}$ & 14 & O. I 133 & 0.1 & 0.1310 & o. I 398 & 0.1484 & 0 & & 39 \\
\hline 1.2 & & & & 0.2 & 0.2 & & I I & & & \\
\hline 1.3 & o. & 0.2 & 0.2776 & & 0.2 & & & & & \\
\hline 1.4 & 0.3365 & $0.343^{6}$ & 50 & 0.3577 & 0.3646 & 0.3 & 0.3784 & 53 & & 38 \\
\hline 1.5 & & & 0.4 I 87 & 3 & 0.4 & & & & & 37 \\
\hline 1.6 & & & 0.4824 & & 0. & & & & & 47 \\
\hline 1.7 & 20 & 65 & 0 & I & & & & & & 2 \\
\hline 1.8 & 78 & & & & & & & & & \\
\hline 1.9 & O. & 0.6 & 0.6523 & 0.6 & o & & 9 & & & \\
\hline 2.0 & 0.6 & 0.6 & 0.7031 & 0.7080 & 0.7 & & 7227 & 75 & 24 & 372 \\
\hline 2.1 & IC & 67 & 514 & $\operatorname{lo}_{0}$ & 8 & & I & & & \\
\hline 2.2 & & & & & & & & & & \\
\hline 2.3 & 0.8329 & & & 0.8 & 0.8502 & & 0.8587 & & & \\
\hline 2.4 & 0.8 & 96 & 0.8838 & 0.8 & 0.8920 & 0.8 & 0.9002 & & 083 & 23 \\
\hline 2.5 & 0.9163 & 03 & .9243 & 0.9 & 0.9322 & 0.9 & 0 & & 78 & 17 \\
\hline 2.6 & 0.9555 & 0.9594 & 0.9632 & 0.9670 & 0.9708 & 0.9 & $0.97^{8} 3$ & & & 0.9895 \\
\hline 2.7 & o. & .9969 & I.0006 & 1.00 & 1.0080 & 6 & 2 & 88 & 5 & 0260 \\
\hline 2.8 & I.C & & 367 & 1.0403 & I.O4 & & & & 78 & 13 \\
\hline 2.9 & I.0647 & 1.0682 & I.07 I 6 & $1.075^{\circ}$ & 1.0784 & I.C & 1.0852 & & I9 & I.0953 \\
\hline 3.0 & I.0986 & I.IOI9 & I. 1053 & 1. 1086 & I. I I I & I. I & I.I I 84 & I.I & 249 & 1. 1282 \\
\hline 3.1 & & & 378 & 0 & & & & & & I. 1600 \\
\hline 3.2 & 1.1632 & 63 & I.I 694 & 725 & & I. & 817 & & 78 & I.1909 \\
\hline 3.3 & I. I939 & I. I969 & 1.2000 & 1.2030 & 1.2060 & 1.2090 & I. 2119 & 1.2149 & 1.2179 & 1. 2208 \\
\hline 3.4 & I.2238 & 1.2267 & I.2296 & & & & & 2 & I. 2470 & \\
\hline 3 & & & & 1.2 & & & 1.2698 & & & \\
\hline 3.6 & 1.2809 & I. 2837 & I. 2865 & I.2892 & I. 2920 & I. 2947 & I. 2975 & 1.3002 & I. 3029 & I.3056 \\
\hline
\end{tabular}




\begin{tabular}{|c|c|c|c|c|c|c|c|c|c|c|}
\hline & O & 1 & 2 & 3 & 4 & 5 & 6 & 7 & 8 & 9 \\
\hline 3.7 & 1.3083 & I.3IIO & 1.3137 & 4 & I.319I & 1.32 & I.3244 & 1.3271 & I.3297 & I. 3324 \\
\hline 3.8 & $1.335^{\circ}$ & 1.3376 & .3403 & I. 3429 & I. 3455 & 1.3481 & I. 3507 & I.3533 & $1.355^{8}$ & I. $35^{84}$ \\
\hline 3.9 & 1.3610 & 1. 3635 & 1.3661 & 1.3686 & 1.3712 & I. 3737 & 1.3762 & 1.3788 & $1.3^{81} 3$ & 1.3838 \\
\hline 4.0 & $1.3^{86} 3$ & 1.3888 & I.39 I 3 & $1.393^{8}$ & I.3962 & 1.3987 & 1.4012 & 1.4036 & 1.4061 & 1.4085 \\
\hline 4.1 & I. 4110 & I. $4 \mathrm{I} 34$ & 1.4159 & 1.4183 & I. 4207 & 1.4231 & I. 4255 & 1.4279 & 1.4303 & 1.4327 \\
\hline 4.2 & I. 4351 & 1.4375 & I. 4398 & 1.4422 & 1. 4446 & 1.4469 & I. 4493 & 1.4516 & I.4540 & 1.4563 \\
\hline 4.3 & 1.4586 & 1.4609 & 1.4633 & 1.4656 & 1.4679 & 1.4702 & I. 4725 & 1.4748 & $1.477^{\circ}$ & 1.4793 \\
\hline 4.4 & 1.4816 & 1.4839 & $1.486 \mathrm{I}$ & 1.4884 & I. 4907 & 1.4929 & I. $495^{1}$ & 1.4974 & I.4996 & I.5019 \\
\hline 4.5 & I. 5041 & I. 5063 & I. 5085 & 1.5107 & 1.5129 & $1.5^{1} 5^{I}$ & 1.5173 & I. 5 I95 & 1.5217 & 1.5239 \\
\hline 4.6 & 1.5261 & 1.5282 & 1.5304 & 1.5326 & I. 5347 & 1.5369 & I. 5390 & I. 5412 & 1.5433 & I. 5454 \\
\hline 4.7 & I. 5476 & I. 5497 & $1.55^{18}$ & I.5539 & $1.55^{60}$ & I. $55^{81} \mathrm{I}$ & 1.5602 & 1.5623 & I. 5644 & 1.5665 \\
\hline 4.8 & I. 5686 & 1.5707 & I. 5728 & I. 5748 & 1.5769 & 1.5790 & 1.5810 & $1.58_{31}$ & 1.5851 & 1.5872 \\
\hline 4.9 & 1.5892 & 1.5913 & I. 5933 & 1.5953 & I. 5974 & I.5994 & 1.6014 & 1.6034 & 1.6054 & I. 6074 \\
\hline 5.0 & I.6094 & 1. 6114 & 1.6r 34 & I.61 54 & I. 6 I 74 & 1.6I94 & 1.6214 & 1.6233 & 1.6253 & 1.6273 \\
\hline 5.1 & 1.6292 & 1.6312 & $1.633^{2}$ & $1.635^{I}$ & $1.637 \mathrm{I}$ & 1.6390 & I.6409 & I.6429 & I. 6448 & 1.6467 \\
\hline 5.2 & 1.6487 & 1.6506 & 1.6525 & 1.6544 & 1.6563 & 1.6582 & 1.6601 & 1.6620 & I.6639 & 1. $665^{8}$ \\
\hline 5.3 & I. 6677 & 1.6696 & 1.6715 & 1.6734 & 1.6752 & 1.6771 & I. 6790 & I. 6808 & 1.6827 & 1.6845 \\
\hline 5.4 & I. 6864 & 1.6882 & 1.6901 & 1.6919 & 1.6938 & $1.695^{6}$ & I.6974 & I.6993 & .7011 & .7029 \\
\hline 5.5 & 1.7047 & I.7066 & I.7084 & 1.7102 & 1.7120 & $1.713^{8}$ & I. 7 I 56 & 1.7174 & 1.7192 & 1.7210 \\
\hline 5.6 & I.7228 & I. 7246 & 1.7263 & 1.7281 & 1.7299 & 1.7317 & I. 7334 & $1.735^{2}$ & 1.7370 & I. $73^{87}$ \\
\hline 5.7 & I. 7 & 1.7422 & 1.7440 & 1.7457 & I.7475 & 1.7492 & I.7509 & 27 & I.7544 & 1.7561 \\
\hline 5.8 & 1.7579 & I.7596 & 1.7613 & 1.7630 & 1.7647 & 1.7664 & I.768I & I.7699 & 1.7716 & I.7733 \\
\hline 5.9 & $1.775^{\circ}$ & 1.7766 & 1.7783 & 1. 7800 & I. 7817 & I. $7^{8} 34$ & $1.785^{1}$ & 1.7867 & 1.7884 & 1.7901 \\
\hline 6.0 & I.79I 8 & I.7934 & 1.7951 & 1.7967 & I. 7984 & 1.8001 & I. 8017 & I. 8034 & $1.805^{\circ}$ & 1.8066 \\
\hline 61 & 1.8083 & I.8099 & 1.8116 & I. $813^{2}$ & I. 8 I 48 & 1.8165 & I.8I $8 \mathrm{I}$ & I.8197 & 1.8213 & 1.8229 \\
\hline 6.2 & I. 8245 & 1.8262 & I. 8278 & I. 8294 & $1.83 \mathrm{IO}$ & 1.8326 & 1.8342 & 1. 8358 & 1.8374 & I. 8390 \\
\hline 6.3 & 1.8405 & 1.8421 & I. 8437 & 1.8453 & 1.8469 & 1.8485 & 1.8500 & 1.8516 & $1.853^{2}$ & I. 8547 \\
\hline 6.4 & 1.8563 & I. 8579 & I. 8594 & 1.86Io & 1.8625 & I. $864 \mathrm{I}$ & 1.8656 & $1.867^{2}$ & I. 8687 & I. $870_{3}$ \\
\hline 6.5 & 1.8718 & 1. 8733 & 1.8749 & I. 8764 & 1.8779 & I.8795 & 1.88 Io & 1.8825 & I. 8840 & 1.8856 \\
\hline 6.6 & 1.8871 & 1.8886 & 1.8901 & 1.8916 & $1.893 \mathrm{I}$ & 1.8946 & I.896I & $1.897^{6}$ & I.899I & 1.9006 \\
\hline 6.7 & I.902 I & 1.9036 & $1.905^{I}$ & 1.9066 & I.908I & 1.9095 & 1.9110 & 1.9125 & 1.9140 & 1.9155 \\
\hline 6.8 & 1.9169 & 1.9184 & 1.9199 & 1.9313 & 1.9228 & 1.9242 & I.9257 & 1.9272 & I.9286 & I.930I \\
\hline 6.9 & 1.9315 & 1.9330 & 1.9344 & I.9359 & 1.9373 & 1.9387 & 1.9402 & 1.9416 & 1.9430 & 1.9445 \\
\hline 7.0 & I.9459 & 1.9473 & I. 9488 & 1.9502 & $1.95^{16}$ & 1.9530 & 1.9544 & I.9559 & 1.9573 & $1.95^{87}$ \\
\hline 7.1 & 1.9601 & I.96I 5 & 1.9629 & 1.9643 & 1.9657 & 1.967 I & I. 9685 & I.9699 & 1.9713 & 1.9727 \\
\hline 7.2 & 1.974 I & 1.9755 & I.9769 & 1.9782 & 1.9796 & I.9810 & 1.9824 & 1.9838 & $1.98_{5} \mathrm{I}$ & 1.9865 \\
\hline 7.3 & 1.9879 & 1.9892 & 1.9906 & 1.9920 & I.9933 & I.9947 & I.996I & I.9974 & $1.99 \$ 8$ & 2.0001 \\
\hline 7.4 & 2.0015 & 2.0028 & 2.0042 & 2.0055 & 2.0069 & 2.0082 & 2.0096 & 2.0109 & 20122 & $2.013^{6}$ \\
\hline 7.5 & 2.0149 & 2.0162 & 2.0176 & 2.0189 & 2.0202 & 2.0215 & 2.0229 & 2.0242 & 2.0255 & 2.0268 \\
\hline 7.6 & $2.028 \mathrm{I}$ & 2.0295 & 2.0308 & 2.0321 & 2.0334 & 2.0347 & 2.0360 & 2.0373 & 2.0386 & 2.0399 \\
\hline 7.7 & 2.0412 & 2.0425 & $2.043^{8}$ & $2.045^{I}$ & 2.0464 & 2.0477 & 2.0490 & 2.0503 & $2.05^{16}$ & 2.0528 \\
\hline 7.8 & 2.0541 & 2.0554 & 2.0567 & 2.0580 & 2.0592 & 2.0605 & 2.0618 & 2.0631 & 2.0643 & 2.0656 \\
\hline 7.9 & 2.0668 & 2.0681 & 2.0694 & 2.0707 & 2.0719 & $2.073^{2}$ & 2.0744 & 2.0757 & 2.0769 & 2.0782 \\
\hline 8.0 & 2.0794 & 2.0807 & 2.0819 & 2.0832 & 2.0844 & 2.0857 & 2.0869 & $2.088 \mathrm{I}$ & 2.0894 & 2.0906 \\
\hline 8.1 & 2.0919 & 2.0931 & 2.0943 & 2.0956 & 2.0968 & 2.0980 & 2.0992 & 2.1005 & 2.1017 & 2.1029 \\
\hline 8.2 & $2.104 \mathrm{I}$ & 2. 1054 & 2.1066 & 2.1078 & 2.1090 & 2.1102 & 2.11114 & 2.1126 & 2.1138 & 2. I I 5O \\
\hline 8.3 & 2.1163 & 2.1 I 75 & 2.1187 & 2.1199 & 2.1211 & 2.1223 & 2.1235 & 2.1247 & $2.125^{8}$ & 2.1270 \\
\hline
\end{tabular}




\begin{tabular}{|c|c|c|c|c|c|c|c|c|c|c|}
\hline & O & 1 & $\mathbf{2}$ & $\mathbf{3}$ & 4 & 5 & 6 & 7 & 8 & 9 \\
\hline 8.4 & 2 & 294 & 6 & 2.1318 & 30 & I 342 & I 353 & 65 & 377 & 89 \\
\hline 8. & OI & 412 & 424 & & 448 & & 471 & 1483 & & \\
\hline 8.6 & I 5 I 8 & 1529 & I $54 \mathrm{I}$ & $2.155^{2}$ & 2.1 564 & .1576 & 2. 1587 & 2.1 599 & .1610 & 2.1622 \\
\hline 8.7 & 1633 & 1645 & 1656 & 2.1668 & 2.1679 & $.169 I$ & I 702 & .1713 & 25 & 2.1736 \\
\hline 8.8 & 48 & 759 & 770 & 1782 & I 793 & I 804 & 815 & 1827 & & \\
\hline 8. & & & & & & I9J 7 & & I939 & 1950 & \\
\hline 9.0 & 2.19 & 2. 1983 & 4 & 06 & 2.2017 & 2028 & 2.2039 & .2050 & & .2072 \\
\hline 9.1 & 2.2083 & 2.2094 & 2105 & 2.2116 & .2127 & $213^{8}$ & $2.214^{8}$ & 9 & 70 & $2.218 \mathrm{I}$ \\
\hline 9.2 & 2.2192 & 2.2203 & 214 & 2225 & .2235 & 2246 & 2257 & .2268 & 79 & 2.2289 \\
\hline 9 & 2.2300 & 2.23 I I & 2.2222 & $2.233^{2}$ & .2343 & 2354 & .2364 & 2375 & & 2.2396 \\
\hline 9.4 & 2407 & 2.2418 & 2.2428 & 2439 & $.245^{\circ}$ & .2460 & 7 & $2.248 \mathrm{I}$ & 92 & 02 \\
\hline 9. & 3 & 23 & & 2544 & 2555 & 2565 & 2576 & 2586 & 597 & 607 \\
\hline 9. & & & & 9 & .2659 & & 30 & 90 & 701 & 2.27 I I \\
\hline 9.7 & 2.2 & 2 & 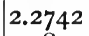 & 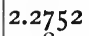 & 2.2762 & 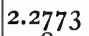 & 2.2783 & 3 & 2803 & $2.28 \mathrm{I} 4$ \\
\hline 9. & 2.2824 & 2.2834 & 2.2844 & 2.2854 & 2.2865 & 2.2875 & 2.2885 & 2.2895 & 2.2905 & 2.2915 \\
\hline 0 & 2.2925 & 2.2935 & 2.2946 & $2.295^{6}$ & .2966 & 2.2976 & 2.2986 & 2.2996 & & 2.3016 \\
\hline 10.0 & 2.3026 & 2.3036 & 2.3046 & $2.305^{6}$ & 2.3066 & 2.3076 & 2.3086 & 2.3096 & 2.3105 & 2.3115 \\
\hline
\end{tabular}




\title{
THE STEAM-TABLE
}

A TABLE OF THE THERMAL AND PHYSICAL PROPERTIES OF SATURATED STEAMVAPOR AND OF THE SPECIFIC HEAT OF WATER

COMPILED FROM VARIOUS SOURCES BY

\author{
SIDNEY A. REEVE \\ PROFESSOR OF STEAM-ENGINEERING AT THE WORCESTER \\ POLYTECHNIC INSTITUTE
}

XYew 19ark

THE MACMILLAN COMPANY

LONDON: MACMILLAN \& CO., LTD.

1903

All rights reserved 
Copyright, I903,

By THE MACMILLAN COMPANY.

Set up and electrotyped January, rgo3.

Norboad 间ess

J. S. Cushing \& Co - Berwick \& Smith

Norwood Mass. U.S.A. 


\section{THE STEAM-TABLE}

\section{NOTES EXPLANATORY OF THE STEAM- TABLE}

A BRIEF investigation of the present knowledge of the thermal properties of water and steam leaves much to be desired. The various authorities are far from agreement. In consulting them, either all except one must be discarded as worthless, or else a highly unscientific compromise or average must be effected in order to recognize them all. The following table represents a combination of both courses. Nothing more can be claimed for it than a conscientious effort after probability of truth.

The British thermal unit in which it is expressed is the specific heat of water at $59^{\circ}$ Fahrenheit or $15^{\circ}$ Centigrade. The mechanical equivalent of heat is taken as 778 . The weight of one cubic inch of mercury is taken as 0.4912 pound.

In making use of the steam-table it is first to be noted that the high temperatures and pressures are at the top and the lower ones at the bottom. The index figures from which the table is to be entered are in pressure and temperature both, and are printed in heavy type in the middle of the page. Either index may be used, according to convenience. From a given index the desired value will be found by passing horizontally to right or left. Immediately above the value sought is printed, in small type, the difference needed to carry the value to that for the next 
integral index above the one chosen and of the same sort. For instance, if it be elected to make use of a pressureindex, and 21 pounds per square inch be the integral pressure involved, opposite 2 I pounds will be found the values for that pressure. Above them are the differences required to carry those values to the correct ones for 22 pounds per square inch, irrespective of the fact that the values for 22 pounds lie several lines above. By the aid of this difference any fractional pressures between $2 \mathrm{I}$ and 22 pounds may easily be interpolated.

The arrangement of the indexes in pressure and temperature side by side will be found to reduce the need for and the work of interpolation to a minimum.

All pressures near or below atmospheric are expressed in inches of mercury-column, thus avoiding the necessity for translation of observations into pounds per square inch; at the same time the gaps which involve the work and the error of interpolation are reduced by half.

The column of logarithms at the right will be found convenient in handling temperature-ratios, both for entropy calculations and for ordinary purposes. While these are themselves logarithms of ratios, it is plain that the difference between any two is itself the logarithm of the ratio of the corresponding temperatures. This column, in fact, reduces all temperature-calculations to the basis of a scale . in which the absolute temperature of melting ice is unity, which is the only natural temperature-scale. 



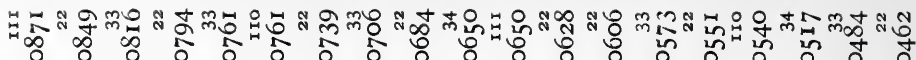

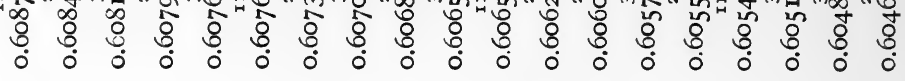

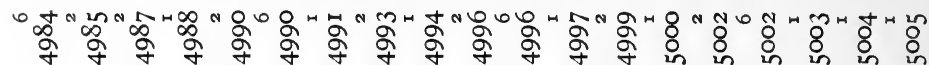

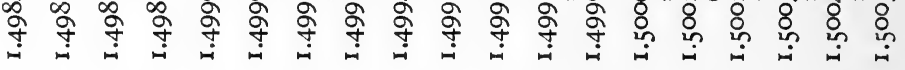

A $8+t+\infty$ n

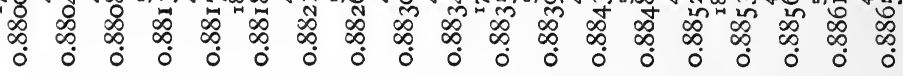

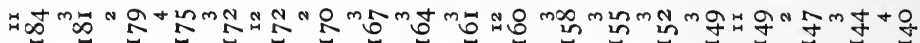

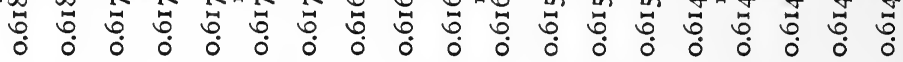

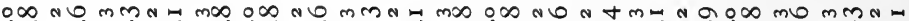

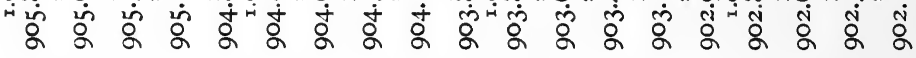

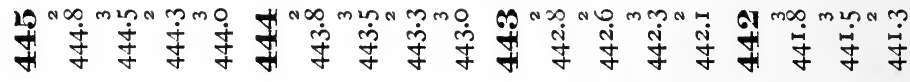

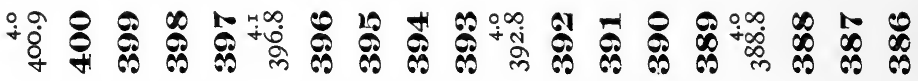

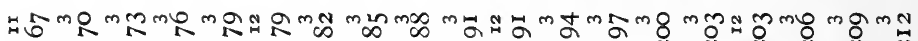

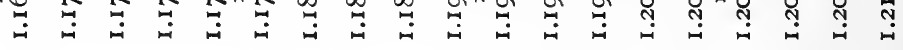

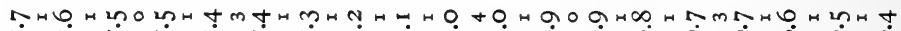

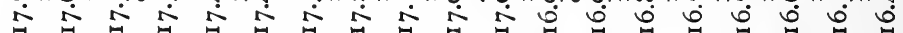

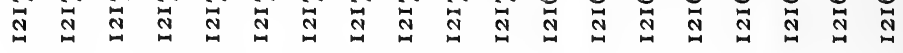

n-

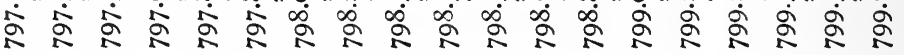

H⿻

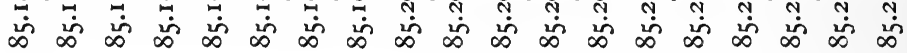

nON" N mH + N

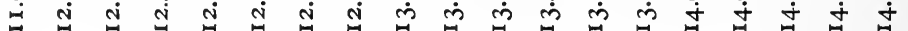

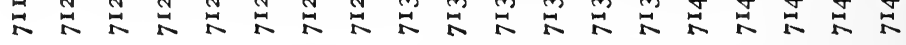

mర

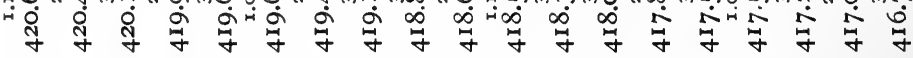

"

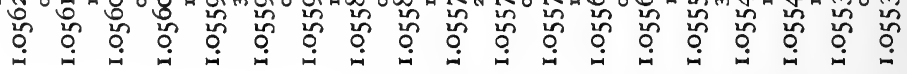




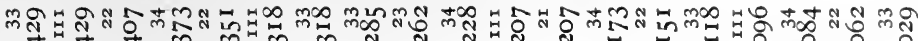

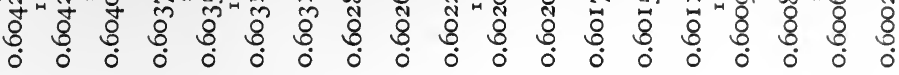
"

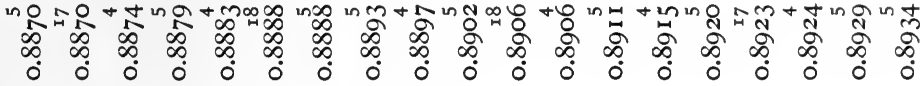

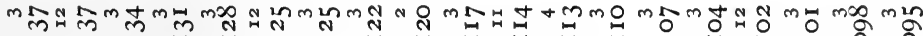

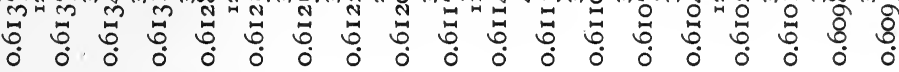

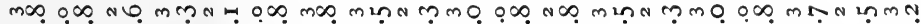
ஃ mo.

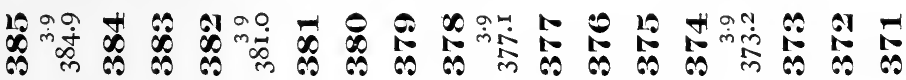

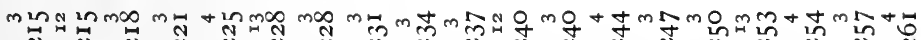
งุ.

H ש+? 芳

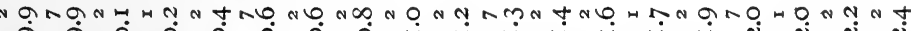

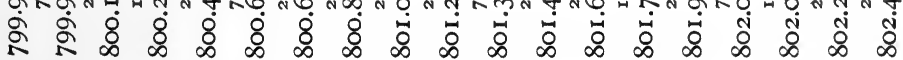

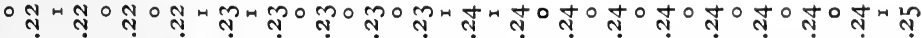
ஸ்

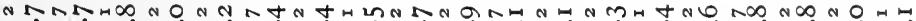

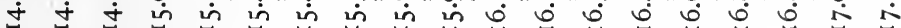

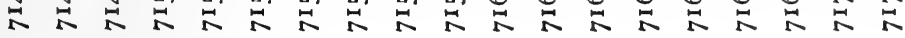

mオ4tn

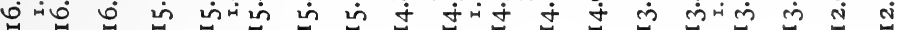
夺

M

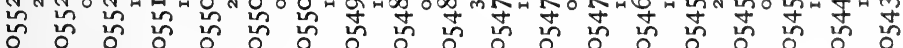
ơ 


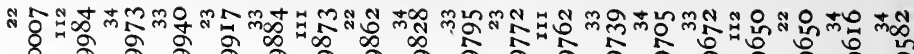
ర유

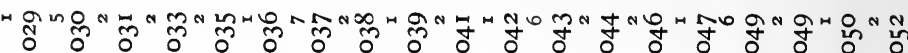

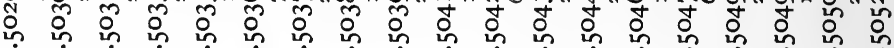

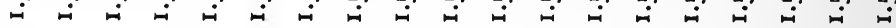

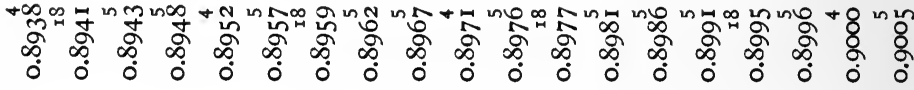

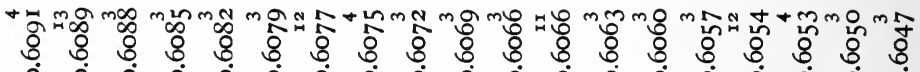

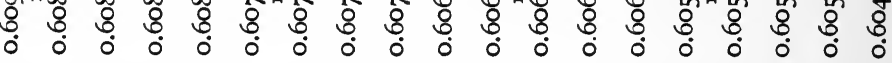

No o̊ mNm+N

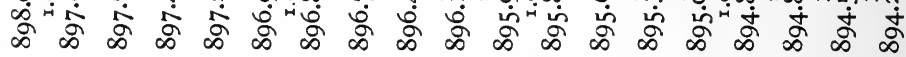

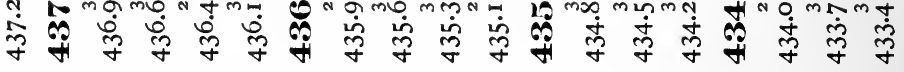

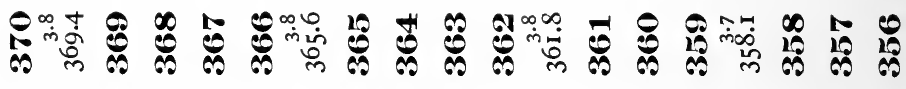

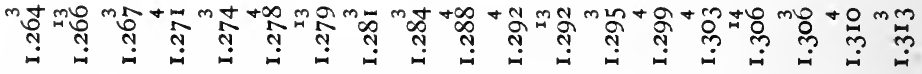
H 胥 N

m Lnob n N

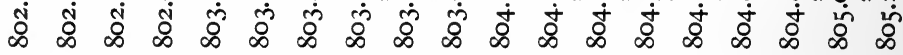

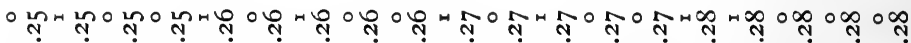

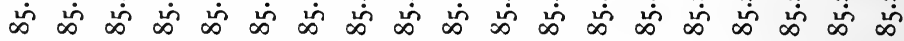

n כot

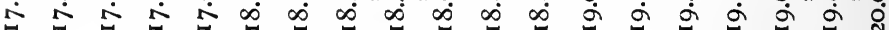

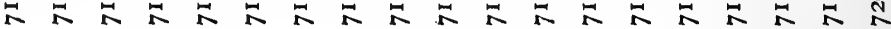

N 岁先

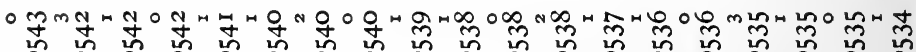

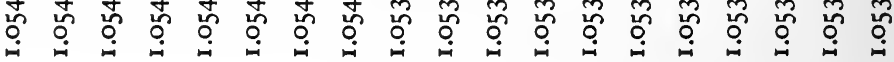


ส 8 ్ㅜ

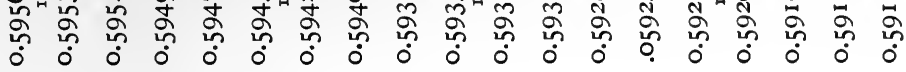

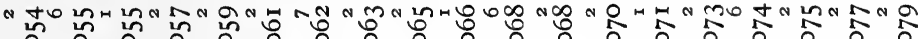

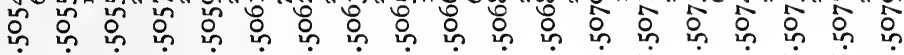

แ 웅 용

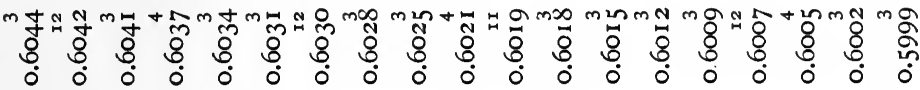

N 0 0, mNmप N m ஓं

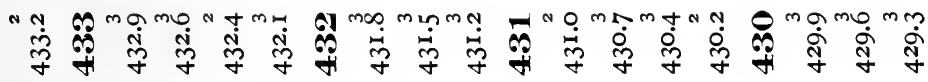

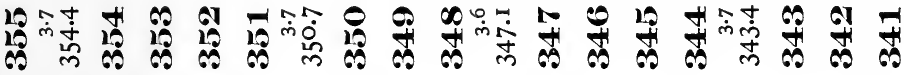

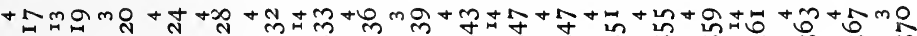

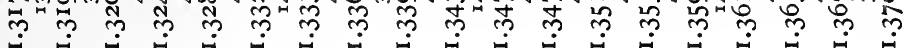

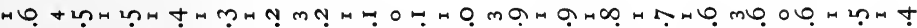

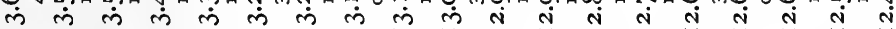
N

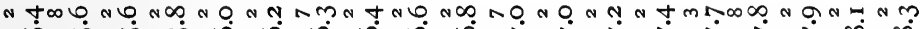

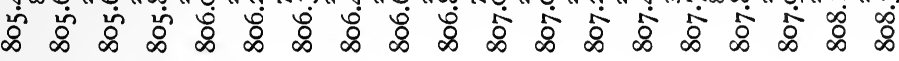

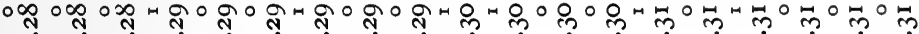
ம்

N

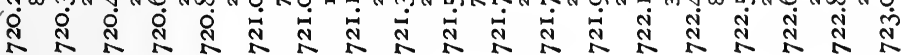

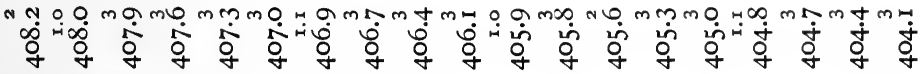

m

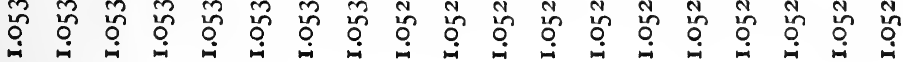




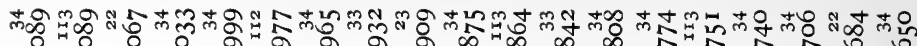

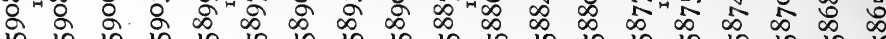

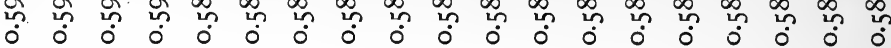

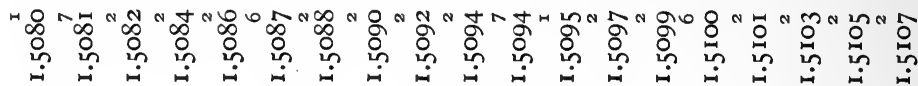

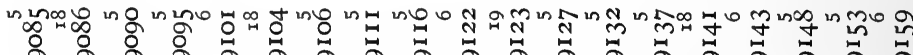
o

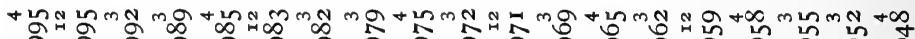

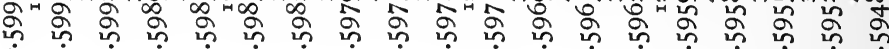

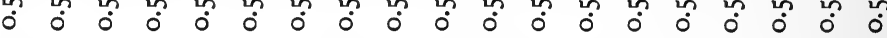

mo 

\begin{tabular}{|c|c|c|c|c|c|c|c|c|c|c|c|c|c|c|c|c|c|}
\hline mo & ô. & \begin{tabular}{l}
$\infty$ \\
$\infty$ \\
\multirow{N}{*}{}
\end{tabular} & min & 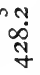 & $\begin{array}{l}\infty \\
01 \\
+1\end{array}$ & $\begin{array}{l}\text { m } \\
\text { n } \\
\text { n }\end{array}$ & $\begin{array}{l}\text { no } \\
\dot{7}\end{array}$ & $\underset{+}{*}$ & $\stackrel{4}{\stackrel{4}{*}}$ & $\begin{array}{l}10 \\
61 \\
4\end{array}$ & \begin{tabular}{l}
$m \infty$ \\
0 \\
\multirow{2}{*}{}
\end{tabular} & mun స్ं & $\begin{array}{l}0 \\
01\end{array}$ & 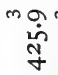 & $\begin{array}{l}\infty \\
\text { ஸ் } \\
\text { + }\end{array}$ & $\begin{array}{l}\text { N } \\
\text { i } \\
+\end{array}$ & 25 \\
\hline 6 & $\begin{array}{l}\infty \\
\dot{m} \\
\dot{m}\end{array}$ & ตे & $\begin{array}{l}\infty \\
0\end{array}$ & की & $\begin{array}{l}m \\
m \\
m\end{array}$ & $\stackrel{\oplus}{0}$ & คै & ติ & ตे & $\begin{array}{c}n \infty \\
\dot{n} \\
m \\
m\end{array}$ & ดे & $\stackrel{\leftrightarrow}{\circ}$ & m & ดี & $\begin{array}{l}\infty \\
01 \\
\infty\end{array}$ & 60 & \\
\hline
\end{tabular}

+ ஐ

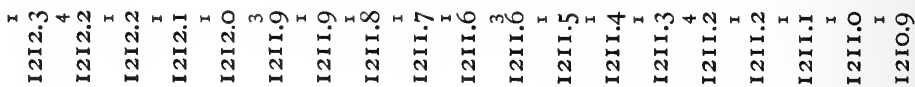

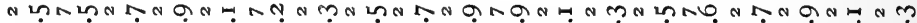

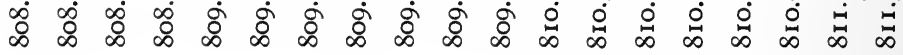

ח

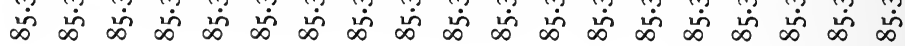

n

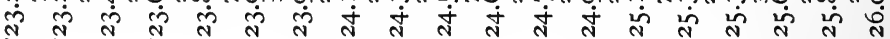

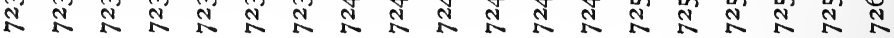

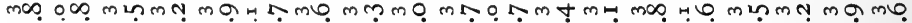
ஸ்

m

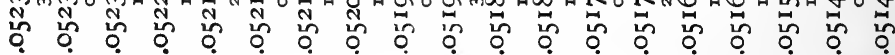
虫 
స్తి 足

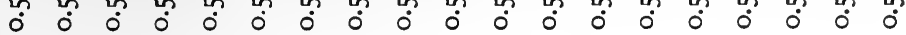

Пิ

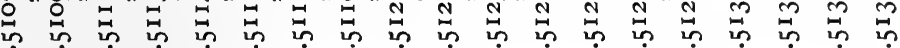

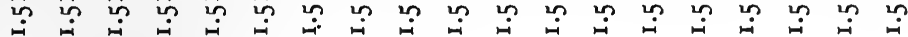

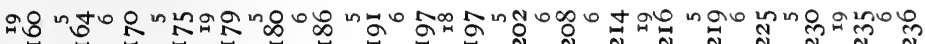

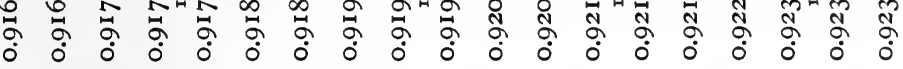

a

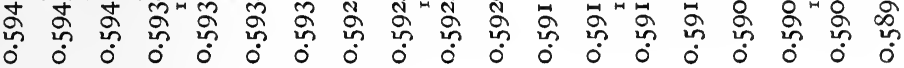

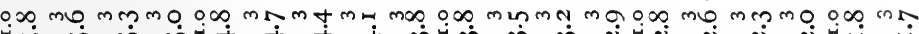

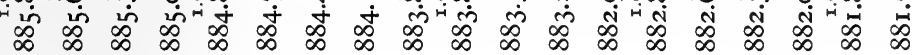

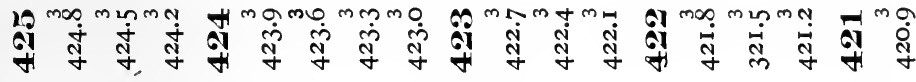

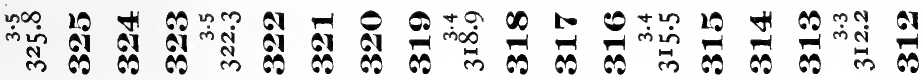

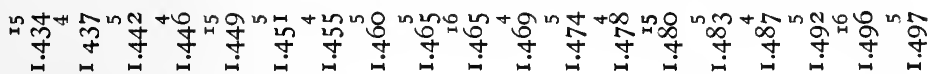

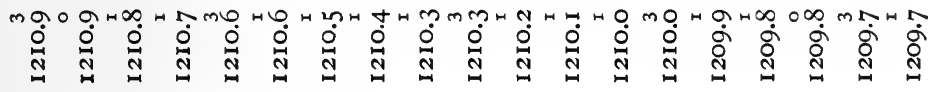

$\infty$ t

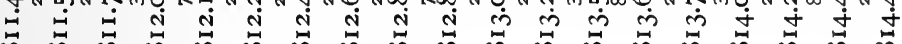
ద

๙ $\dot{\infty} \dot{\infty} \dot{\infty} \dot{\infty} \dot{\infty} \dot{\infty} \dot{\infty} \dot{\infty} \dot{\infty} \dot{\infty} \dot{\infty} \dot{\infty} \dot{\infty} \dot{\infty} \dot{\infty} \dot{\infty} \dot{\infty} \dot{\infty} \dot{\infty}$

$\infty \pi$ a n 4 , N

O ஓ " 


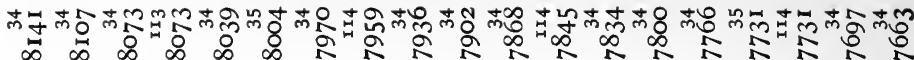

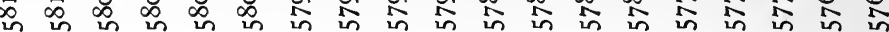

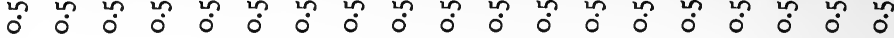

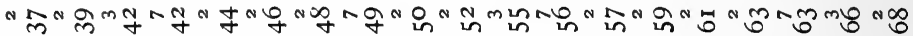
川 थ

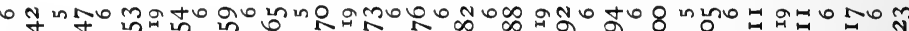
ก

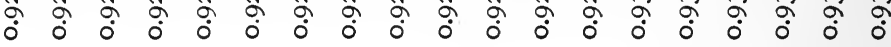

$+น n m N$ m

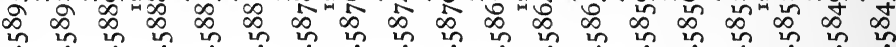
$\begin{array}{lllllllllllllllllll}0 & 0 & 0 & 0 & 0 & 0 & 0 & 0 & 0 & 0 & 0 & 0 & 0 & 0 & 0 & 0 & 0 & 0 & 0\end{array}$

$m+m \backsim m \infty 0, \infty$ munN mọ

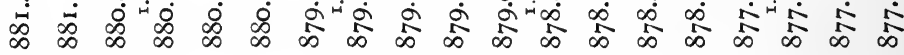

mo mmmo

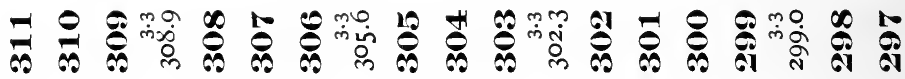

แn $N$ แn+

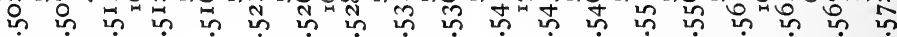

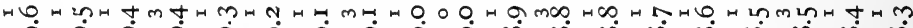
이 ᄋ

mกN GNmNm N 范

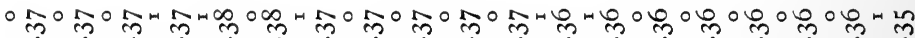
$\dot{\infty} \dot{\infty} \dot{\infty} \dot{\infty} \dot{\infty} \dot{\infty} \dot{\infty} \dot{\infty} \dot{\infty} \dot{\infty} \dot{\infty} \dot{\infty} \dot{\infty} \dot{\infty} \dot{\infty} \dot{\infty} \dot{\infty} \dot{\infty} \dot{\infty}$

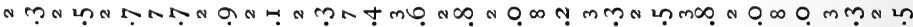

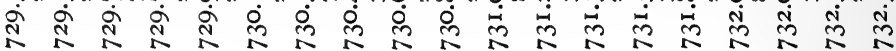

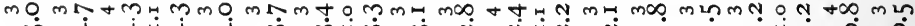

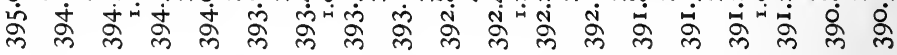
" ก-

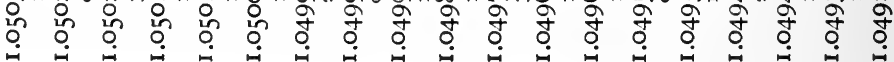




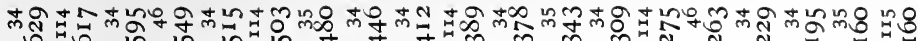

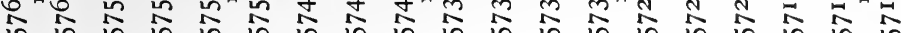

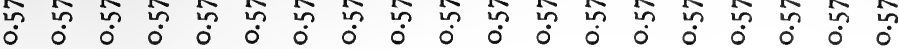

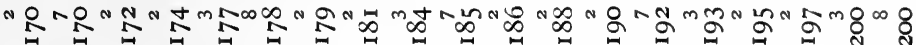

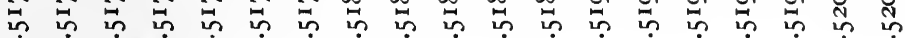
山

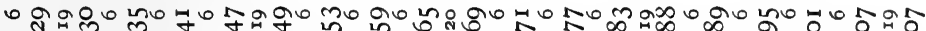

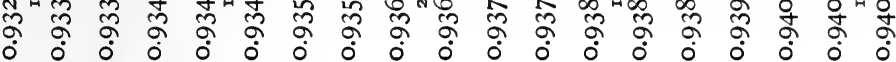

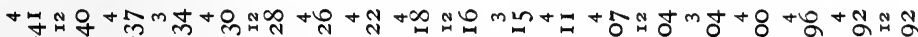

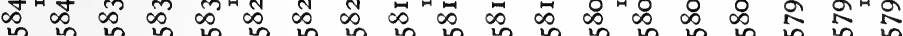

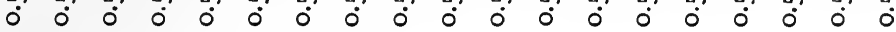

ma 0 mo +N ma 000 mo mmmo 0, mNm+mm

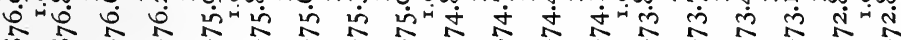

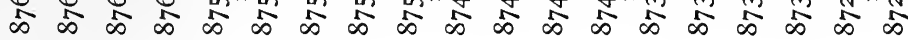

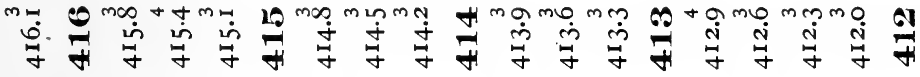

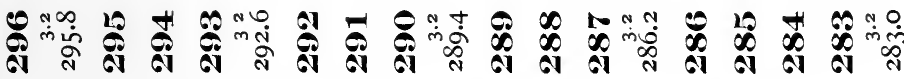

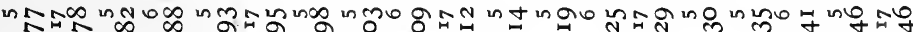
굴 थ m

N O N" m

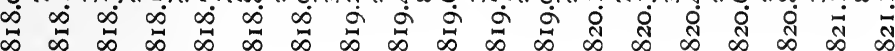

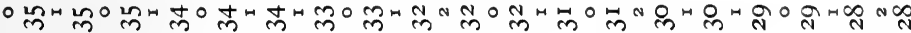
$\dot{\infty} \dot{\infty} \dot{\infty} \dot{\infty} \dot{\infty} \dot{\infty} \dot{\infty} \dot{\infty} \dot{\infty} \dot{\infty} \dot{\infty} \dot{\infty} \dot{\infty} \dot{\infty} \dot{\infty} \dot{\infty} \dot{\infty} \dot{\infty} \dot{\infty}$

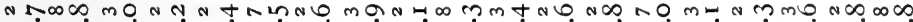

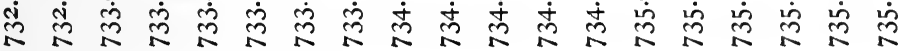

mN. H $+\infty$ mथmN OH m

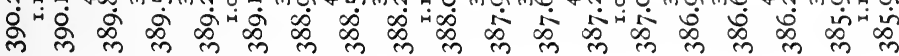

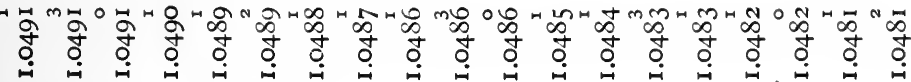




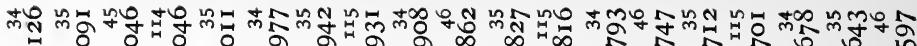

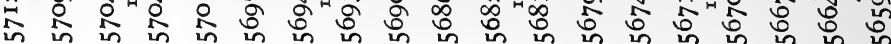

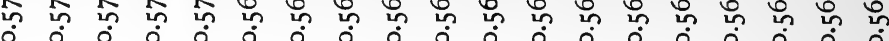

N రู N ก

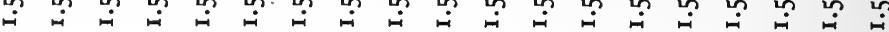

N

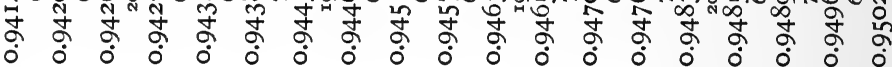

$-100$

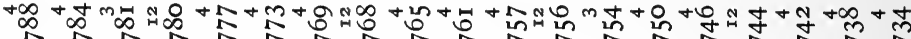
in in in in in in in in in in in in in in in $\begin{array}{r}n \\ 0\end{array}$ in in in $\begin{array}{llllllllllllllllllllllll}0 & 0 & 0 & 0 & 0 & 0 & 0 & 0 & 0 & 0 & 0 & 0 & 0 & 0 & 0 & 0 & 0 & 0 & 0\end{array}$

mum N $+\infty$ :

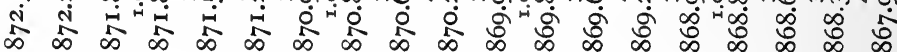

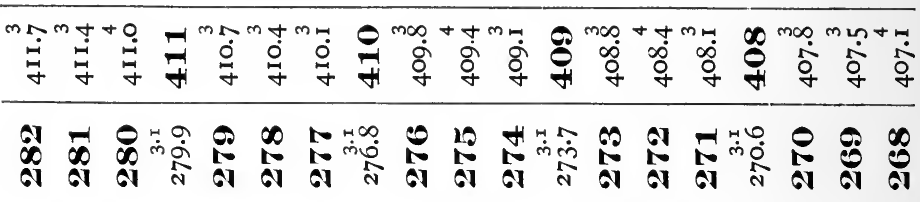

○ N in 0 의

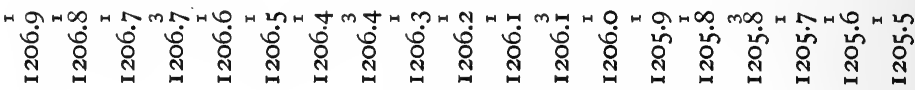
N ?

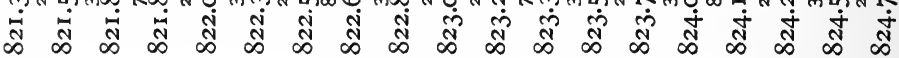

m N

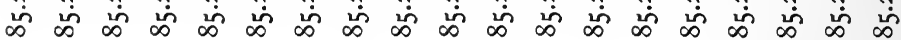

mm NMN MNMNNm N N

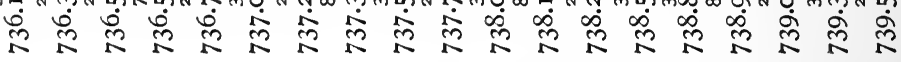
mo $+\mathrm{N}$ -

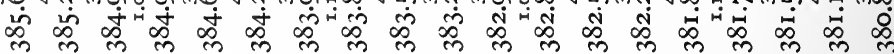
-

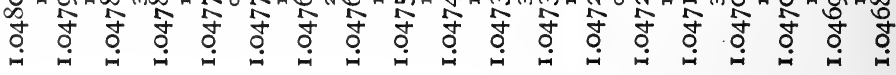




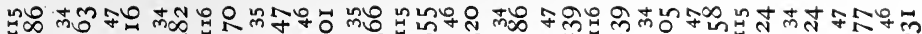
น นn นn

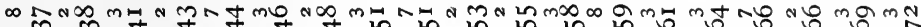

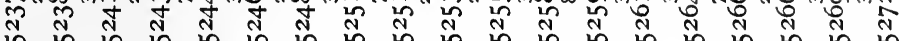

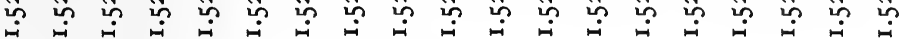

\& กำ แกत

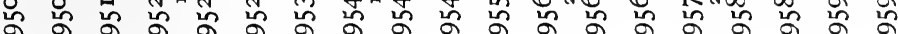

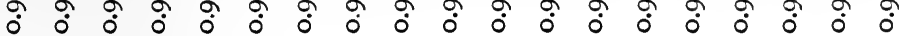

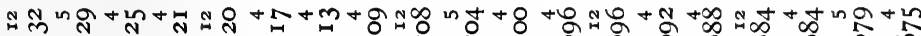
ก 4 ก

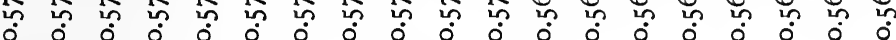

$0 \infty m$ m + m m

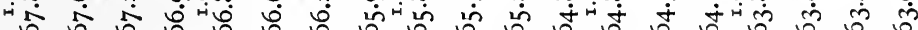
ஸे

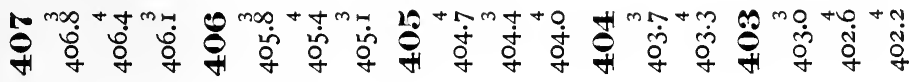

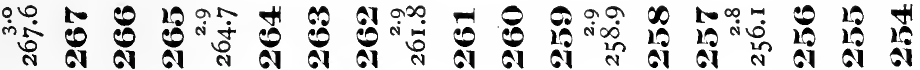

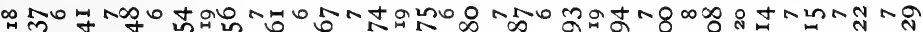
ஸ

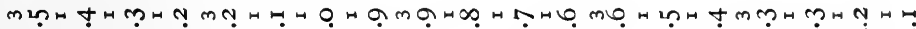

㲾

nom mo n

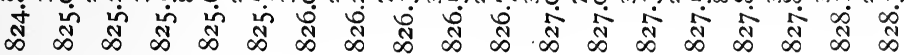

HO OO O OHW N

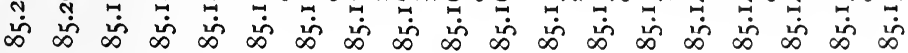

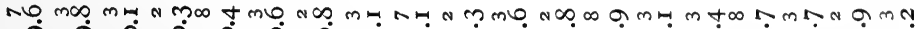
๙ூ

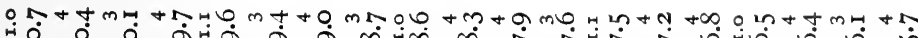
-

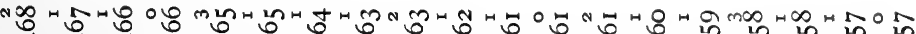
齐 


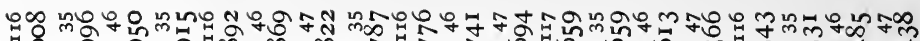

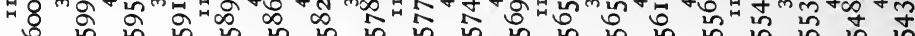

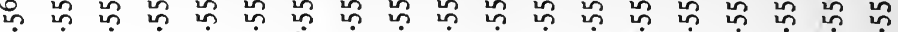
$\begin{array}{llllllllllllllllllllll}0 & 0 & 0 & 0 & 0 & 0 & 0 & 0 & 0 & 0 & 0 & 0 & 0 & 0 & 0 & 0 & 0 & 0 & 0\end{array}$

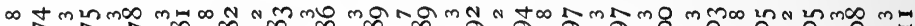
กิ N N N N

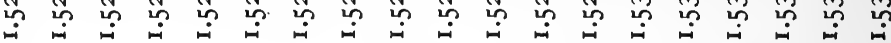

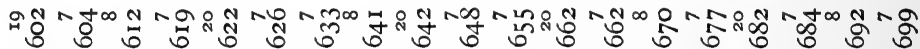

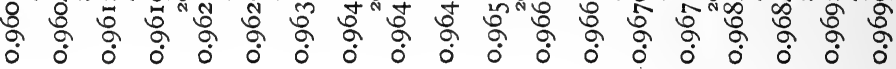

N N n

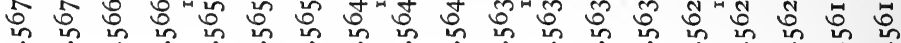

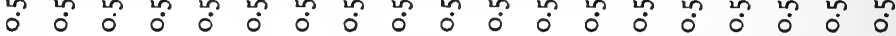

$0 \infty m$ m ن

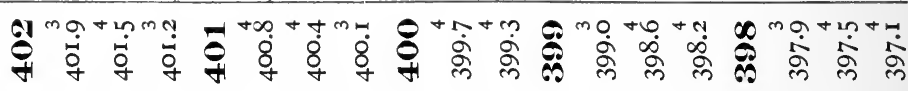

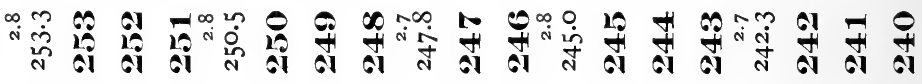

\& 雨 mOுH 总

M 2 m

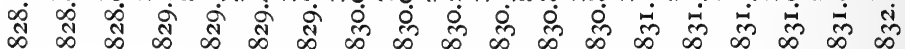

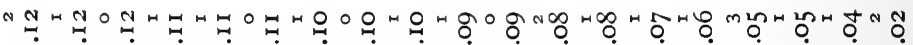

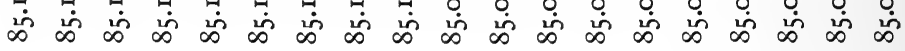

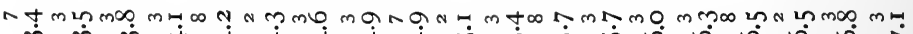

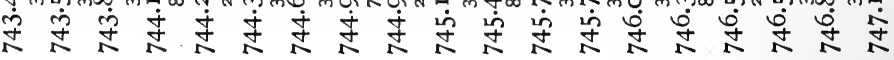

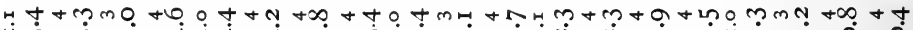

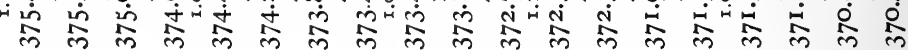

N 晸 


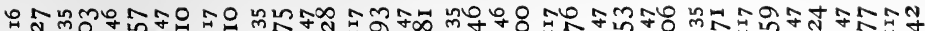

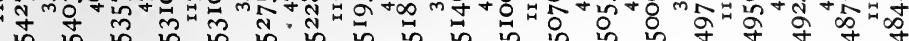

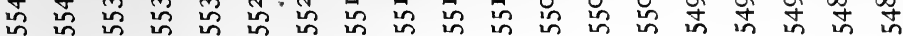

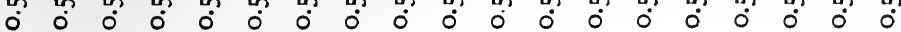

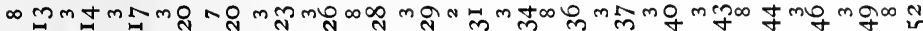

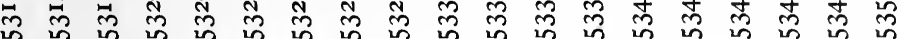

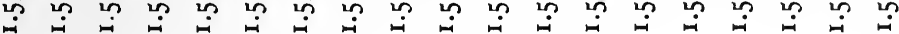

\& 合

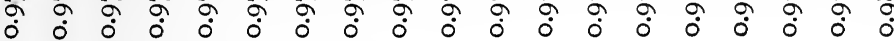

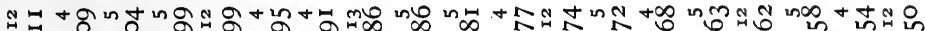
b

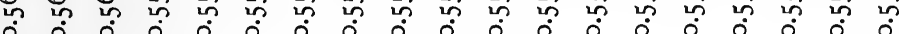

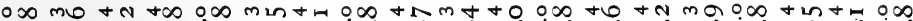

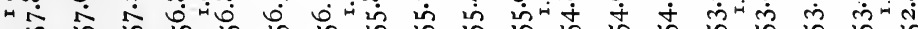

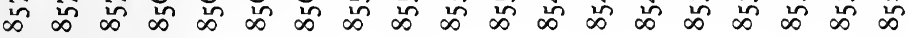

ம

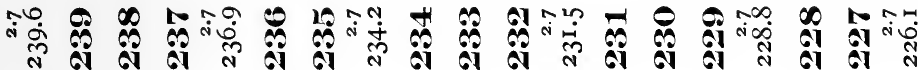

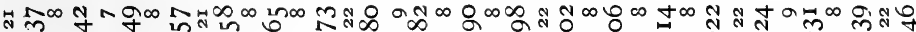

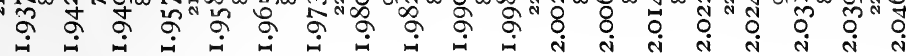

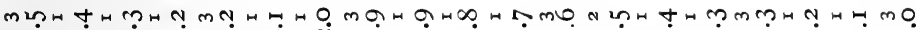

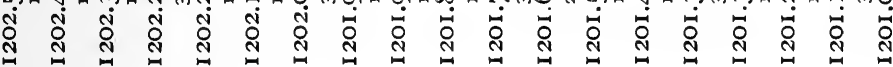

NN m t m mo

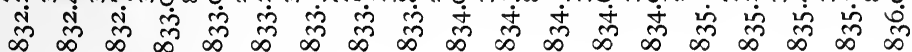

m

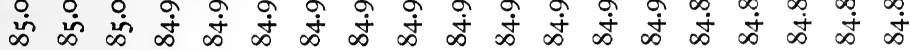

NN m+mNmo

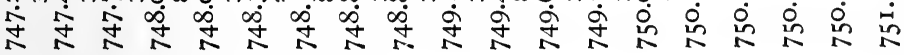

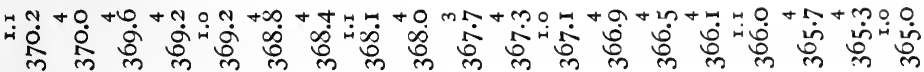

no

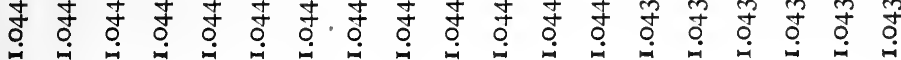




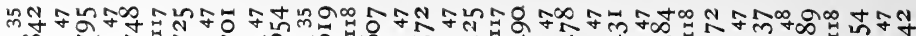

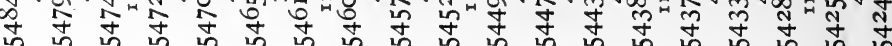

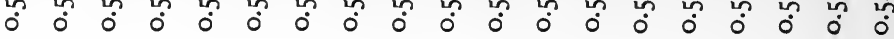

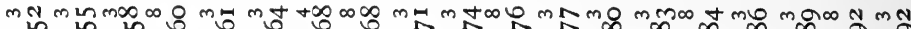

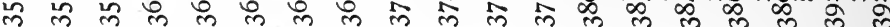
นิ

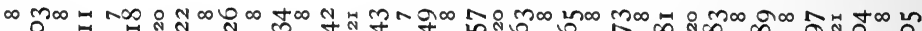

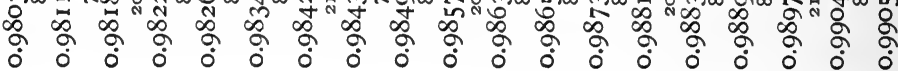

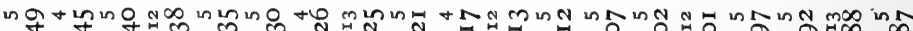

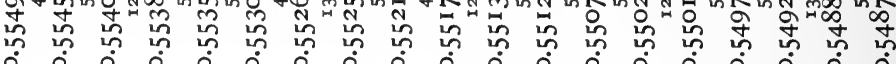

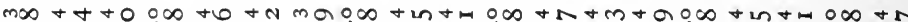
岗

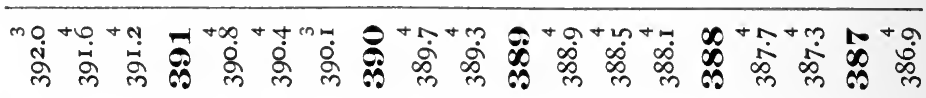

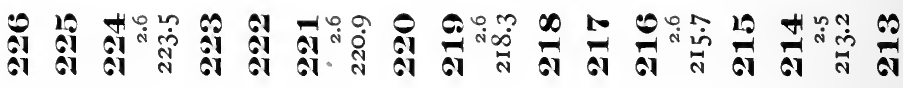

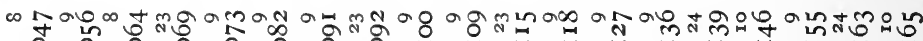

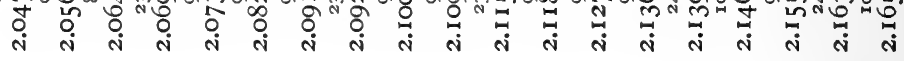

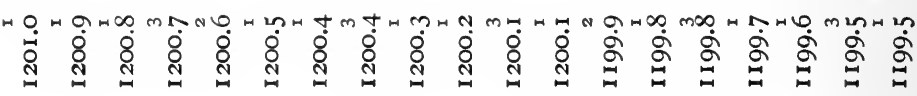

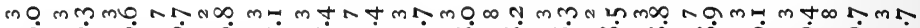

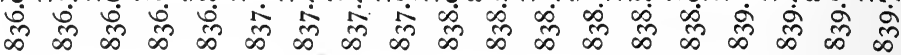

舟品

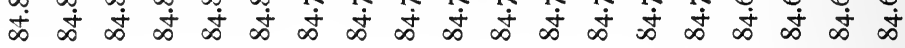

mN m n m

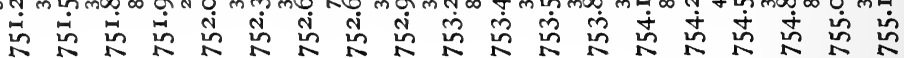

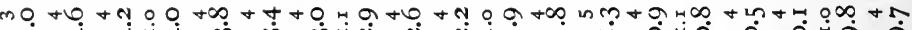

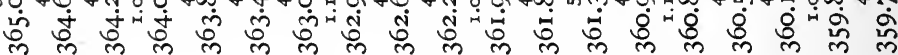

mo

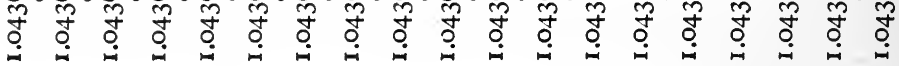




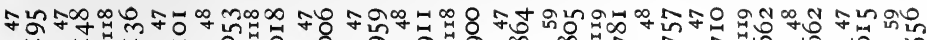

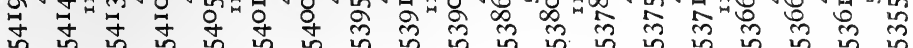

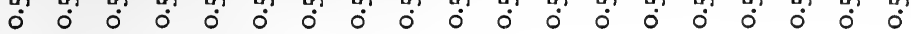

+b mgा

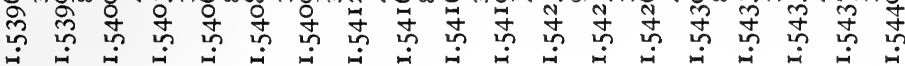

$\infty$ m⿻ $^{2}$ ส है

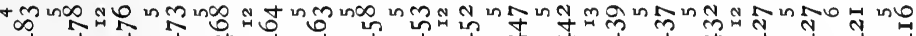

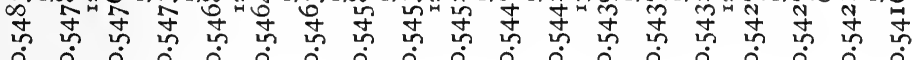
$\begin{array}{lllllllllllllllllll}\circ & 0 & 0 & 0 & 0 & 0 & 0 & 0 & 0 & 0 & 0 & 0 & 0 & 0 & 0 & 0 & 0 & 0 & 0\end{array}$

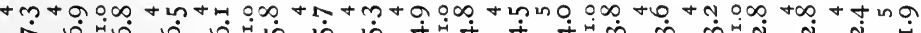
市

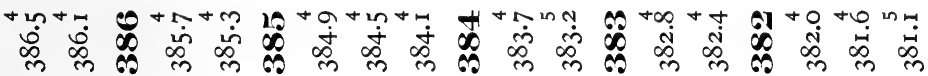

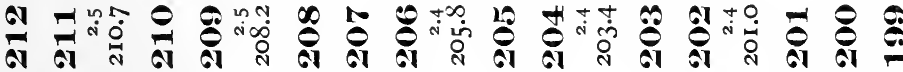

व

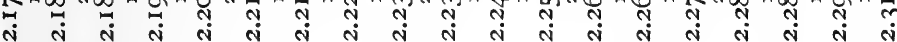

N mhN

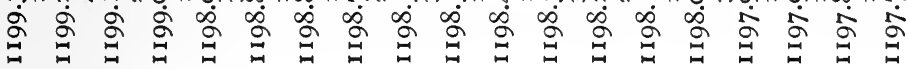

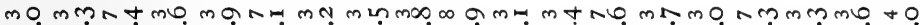
守

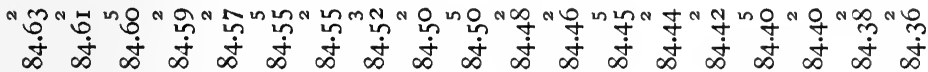

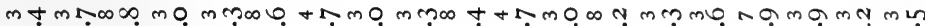

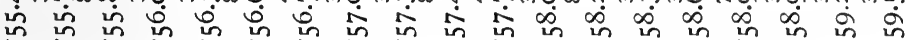

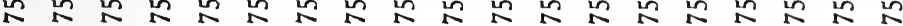

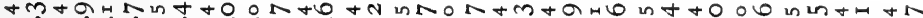
ஸे

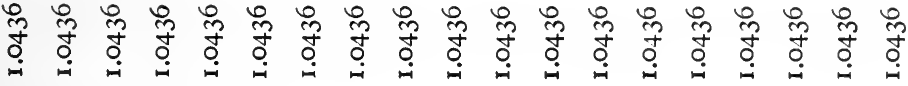




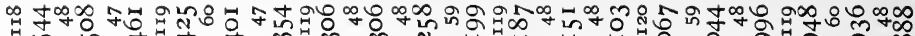

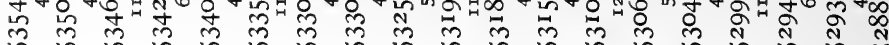

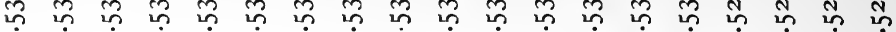
$\begin{array}{lllllllllllllllllllllll} & 0 & 0 & 0 & 0 & 0 & 0 & 0 & 0 & 0 & 0 & 0 & 0 & 0 & 0 & 0 & 0 & 0 & 0 & 0\end{array}$

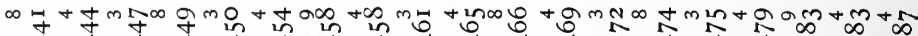

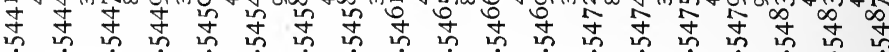

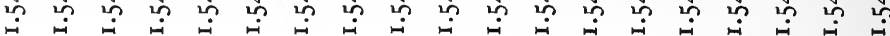

처ำ 每

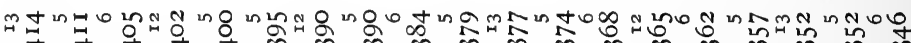
में $\begin{array}{llllllllllllllllllllllllllll}0 & 0 & 0 & 0 & 0 & 0 & 0 & 0 & 0 & 0 & 0 & 0 & 0 & 0 & 0 & 0 & 0 & 0 & 0\end{array}$

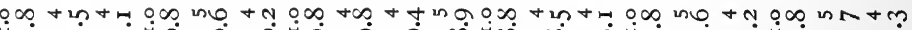
ॠ

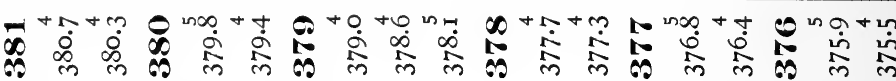

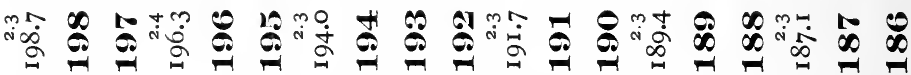

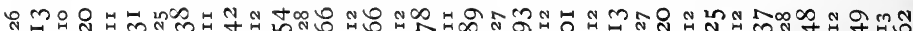
ก

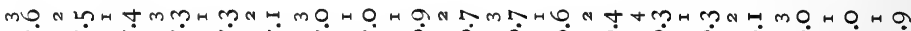

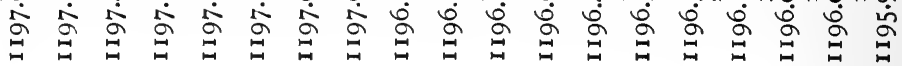

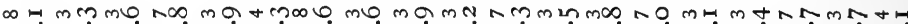
寺 m ஐ

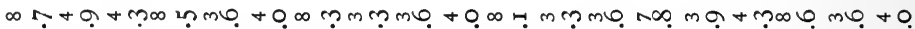

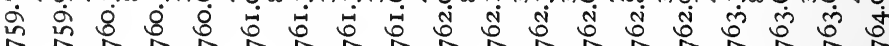
N N N N N

H n n

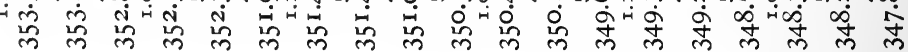

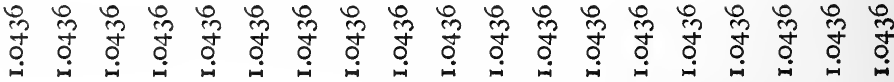




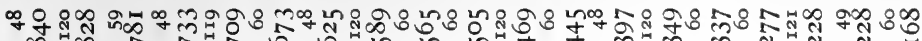

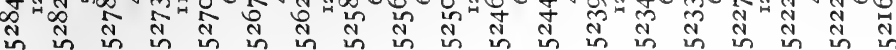
กิ

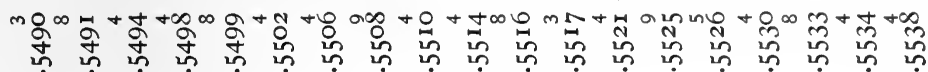

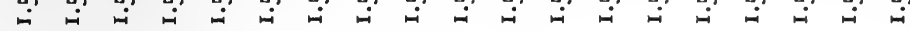

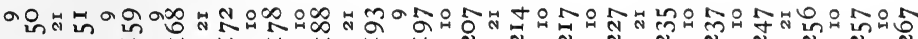
○్-

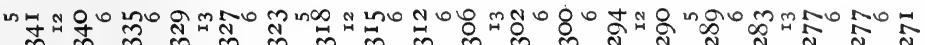

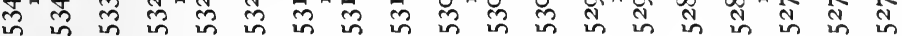

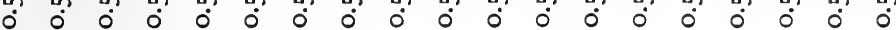

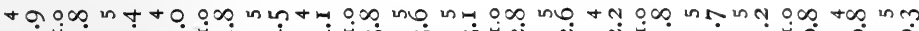

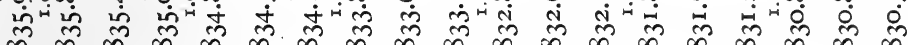

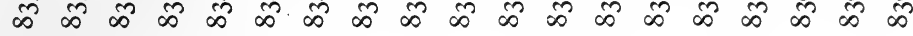

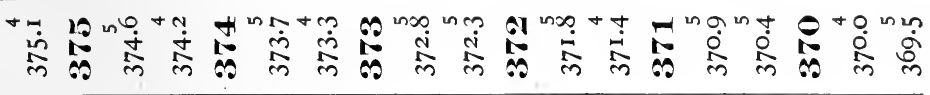

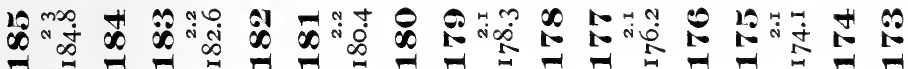

N

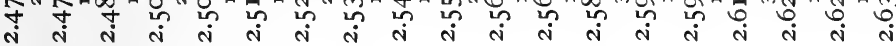

mo mNNO M M in

$m+\infty$ m

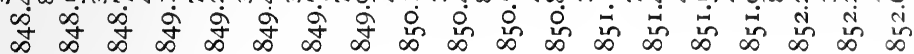

N 음

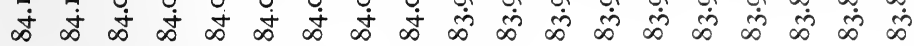

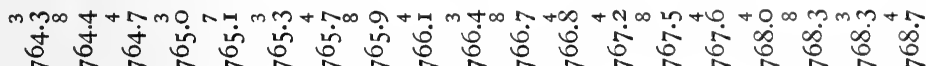

D.

m m

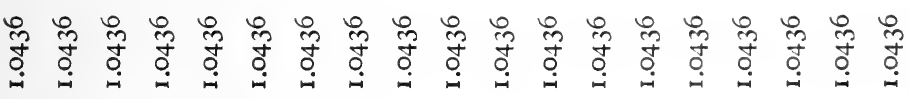




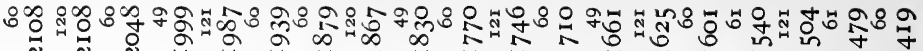

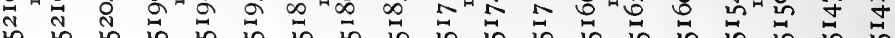

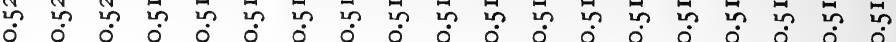

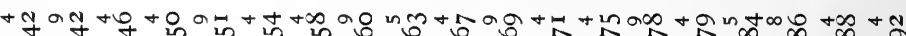

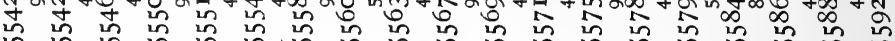

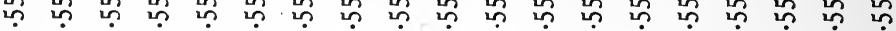

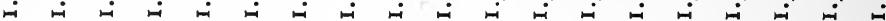

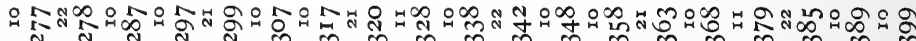

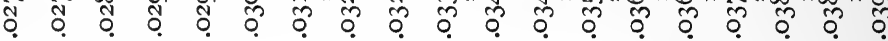
- M

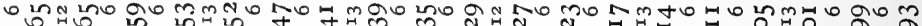

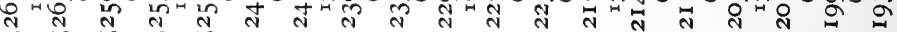

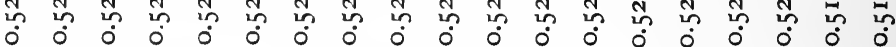

un o, n ?

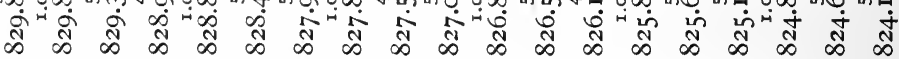

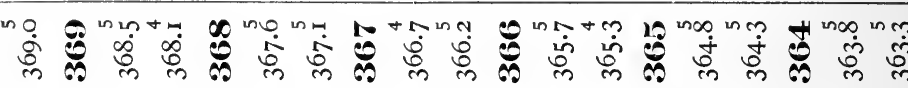

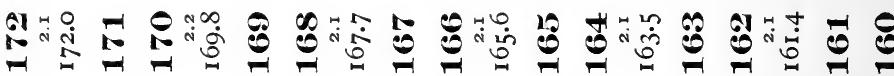

t

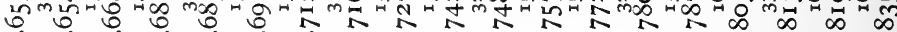

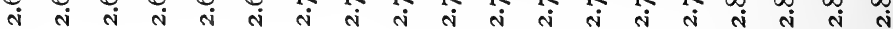

H ஸे

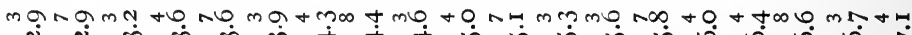

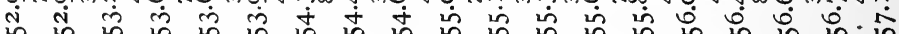

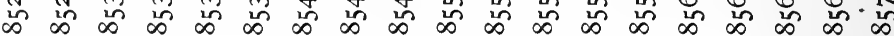

N $\dot{\infty} \dot{\infty} \dot{\infty} \dot{\infty} \dot{\infty} \dot{\infty} \dot{\infty} \dot{\infty} \dot{\infty} \dot{\infty} \dot{\infty} \dot{\infty} \dot{\infty} \dot{\infty} \dot{\infty} \dot{\infty} \dot{\infty} \dot{\infty} \dot{\infty}$

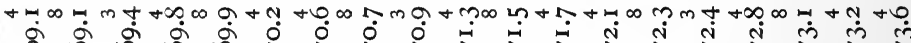

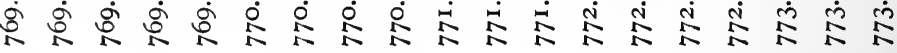

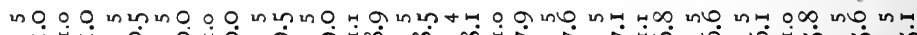

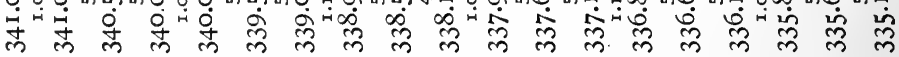

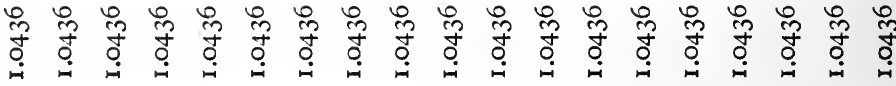




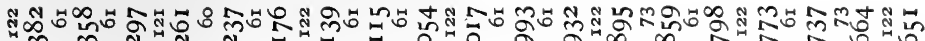
M N

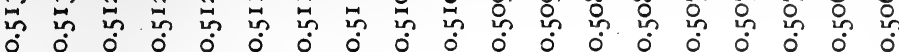

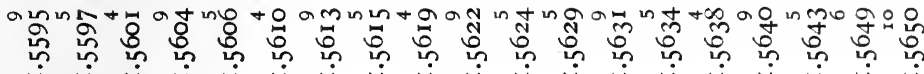

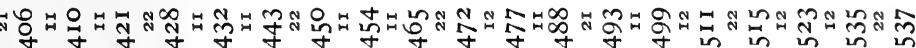
茴

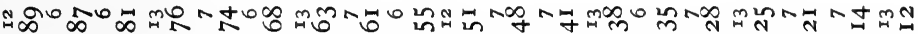

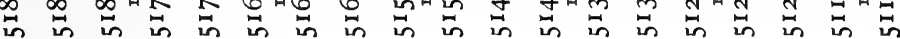

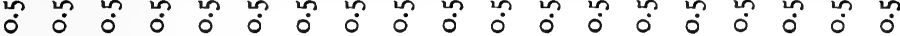

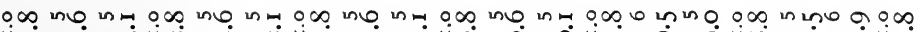

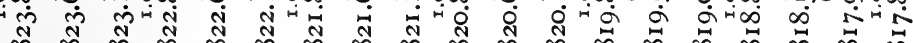

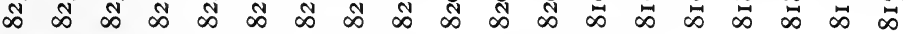

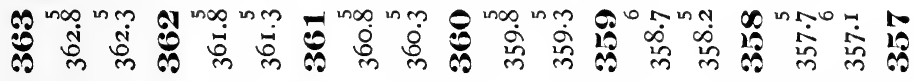

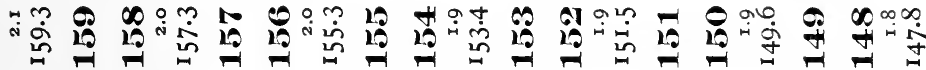

mర్t

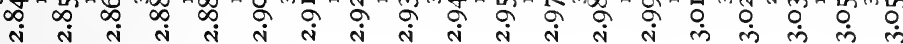

लं

nm+

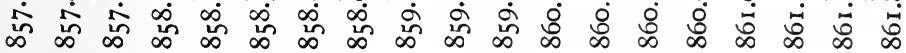

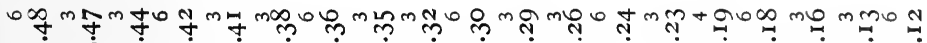

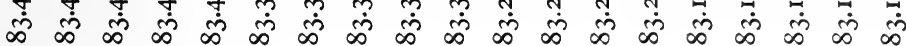

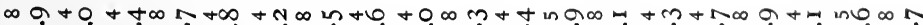

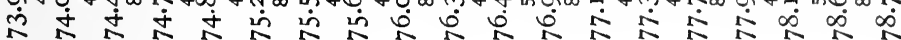

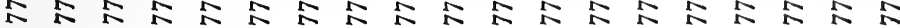

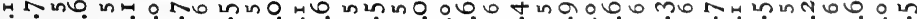

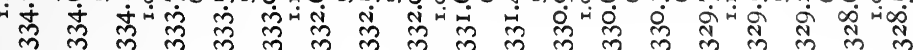

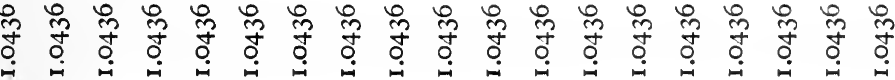




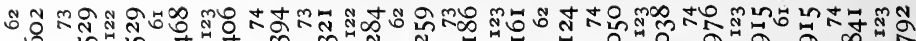

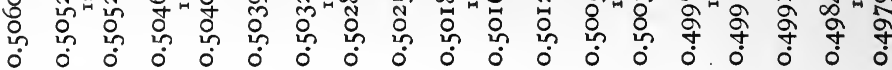

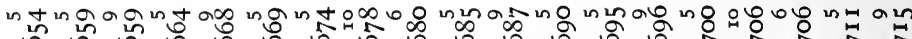
โ

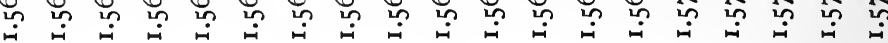

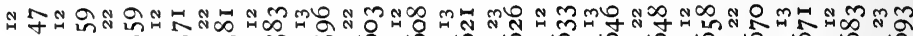

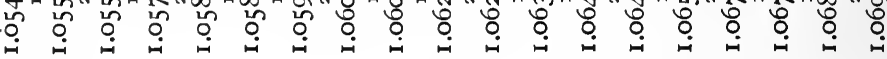

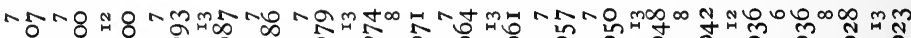
군 นิ

n $+0 \infty$ o, nm

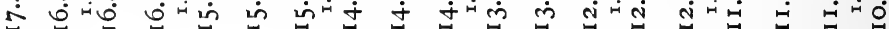

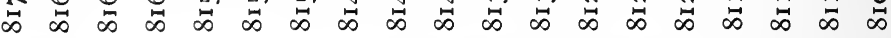

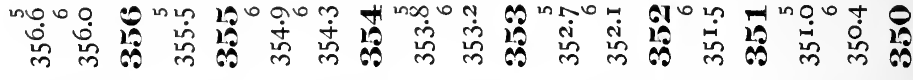

\section{I}

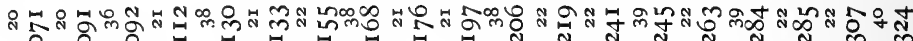
$\dot{m} \dot{m} \dot{m} \dot{m} \dot{m} \dot{m} \dot{m} \dot{m} \dot{m} \dot{m} \dot{m} \dot{m} \dot{m} \dot{m} \dot{m} \dot{m} \dot{m}$

N 罥

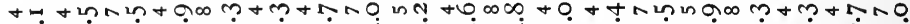
ஸे

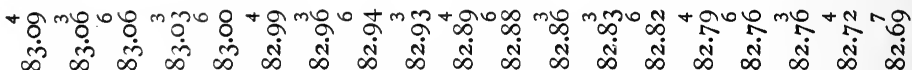

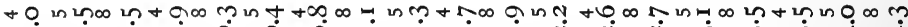
N

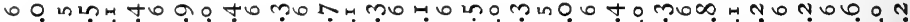

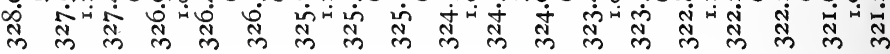

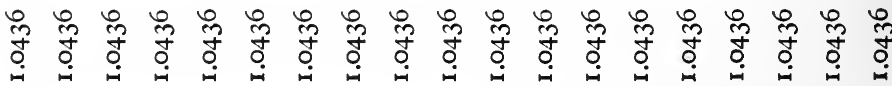




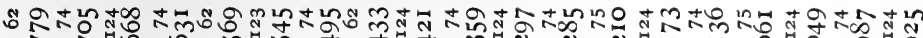

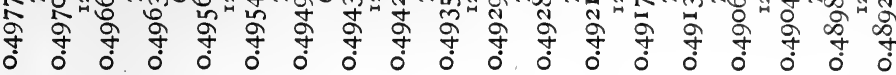

mo N N $N$ N N N N

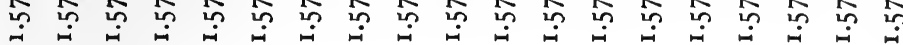

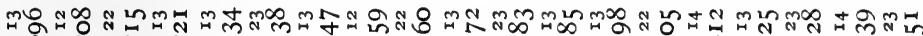

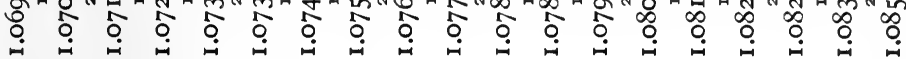

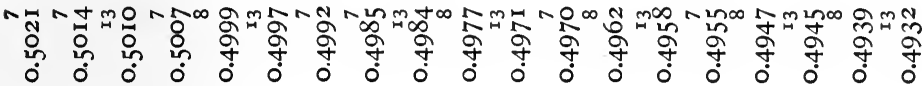

แN ○ ஊ

\section{ค่}

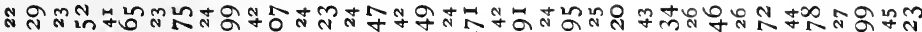
$m$ m $m$ m $\dot{m} \dot{m} \dot{m} \dot{m} \dot{m} \dot{m} \dot{m} \dot{m} \dot{m} \dot{m} \dot{m} \dot{m} \dot{m}$

H

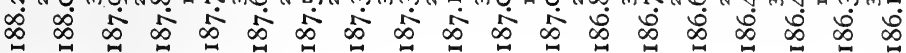

th n

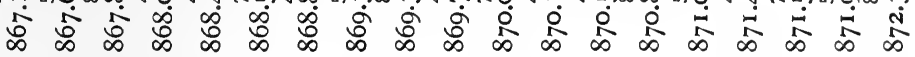

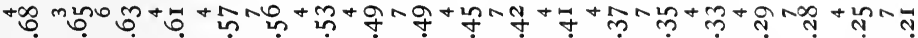

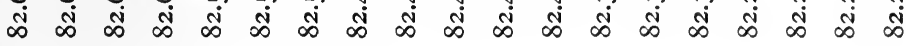

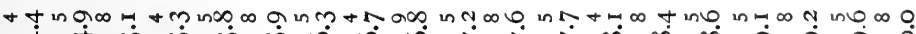
寍

о о ичн

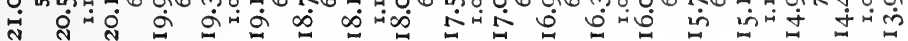

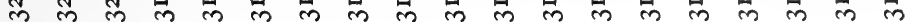

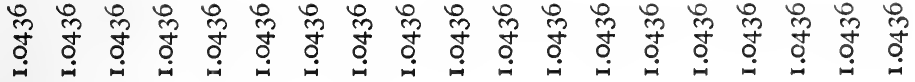




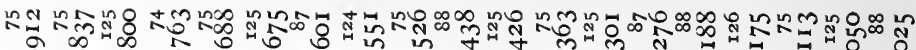

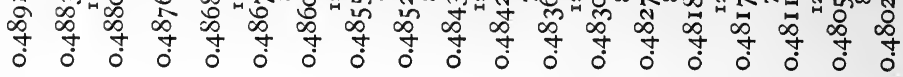

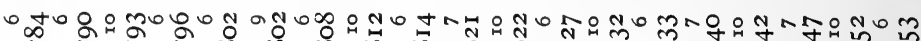
全 น น น น น

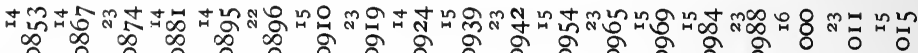
ర్త

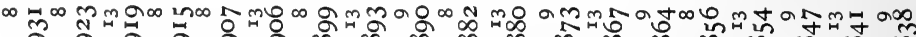

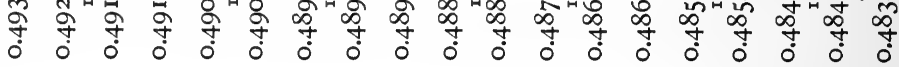

ONG ஓ ரீ

कू⿻ @i

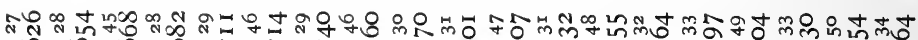

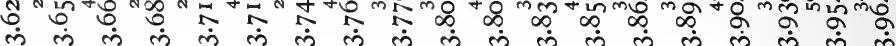

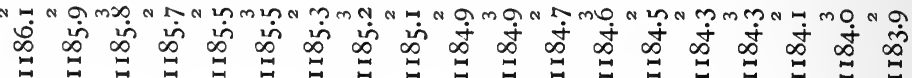

+

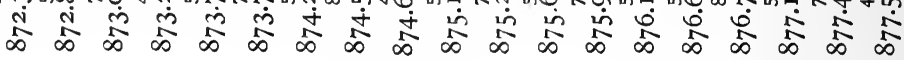

+ ก

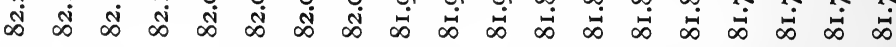

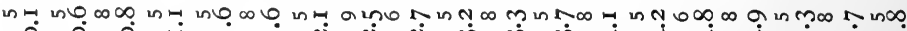

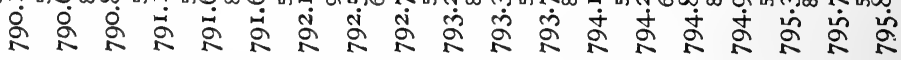

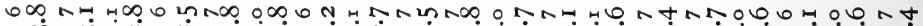

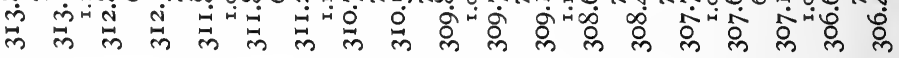

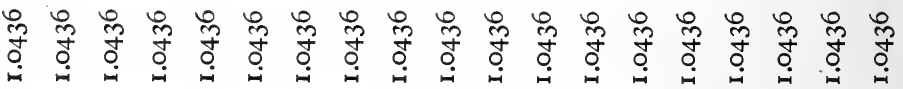


œ నై

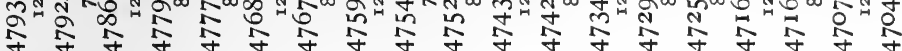

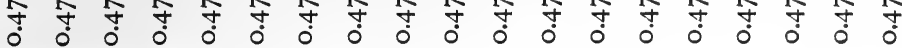

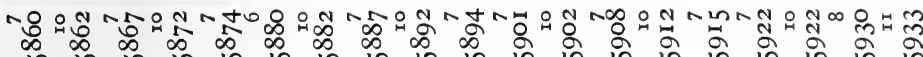
此 -

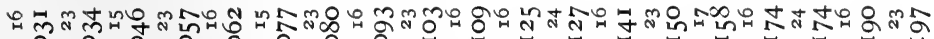

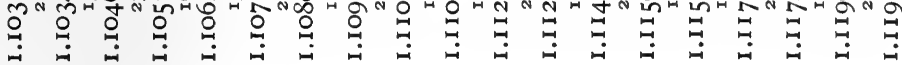

a요에

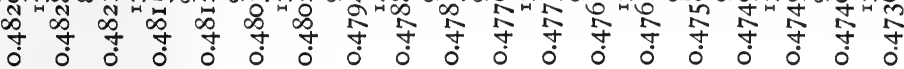

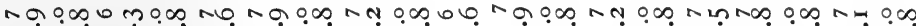
in

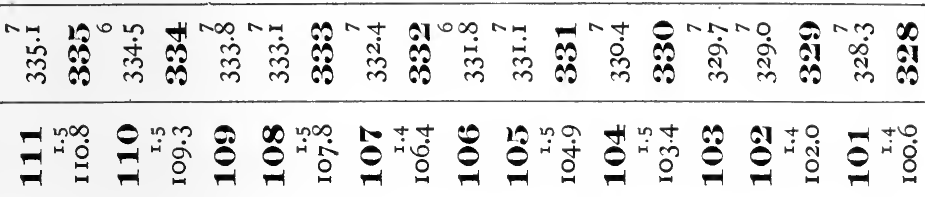

mू 응 of

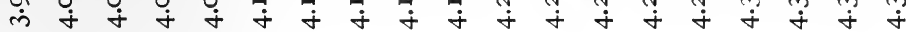

N NmNa ?m

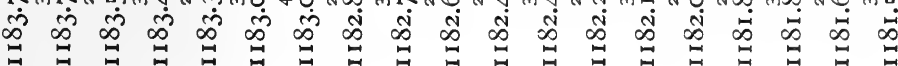

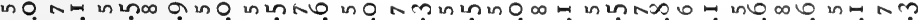

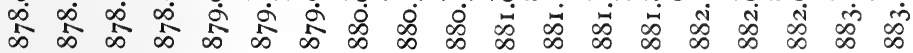
आ

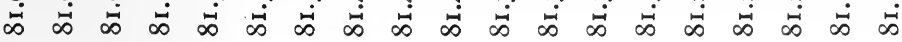

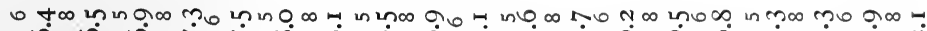

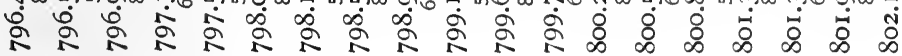

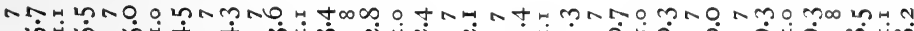
ஸें

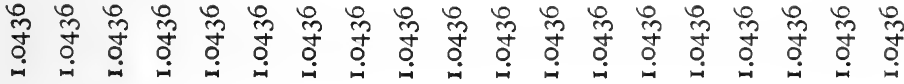




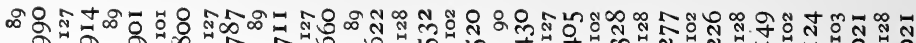
0
0
0

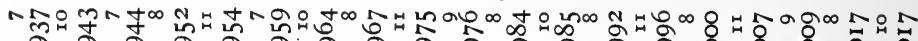

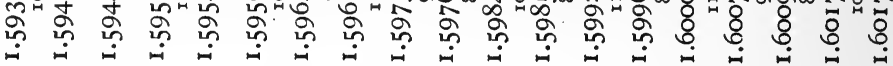

औरำ

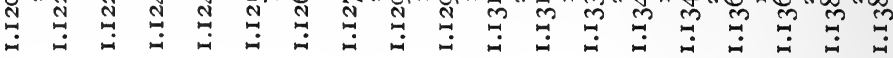

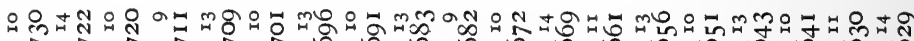

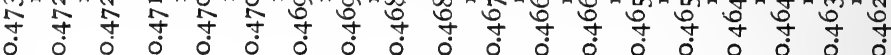

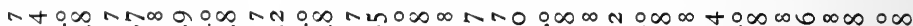

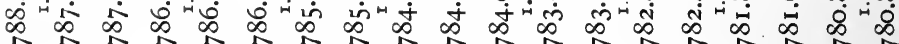

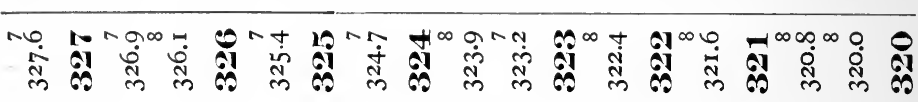

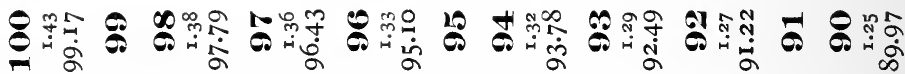

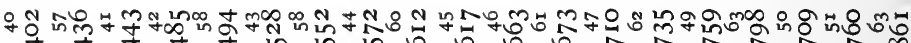

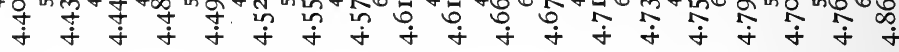

n tmann mam ఉ no ก

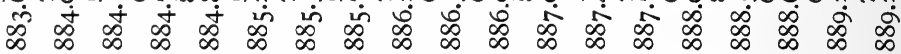

แ

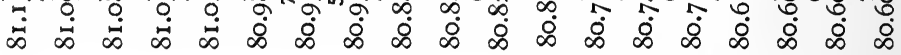

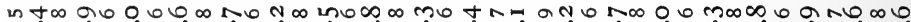
ஸे

No o

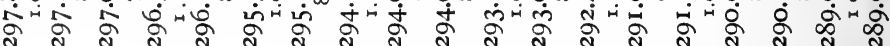

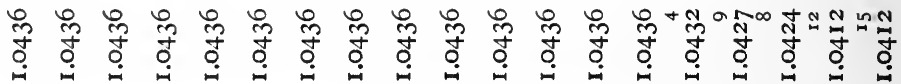



윰워 尔

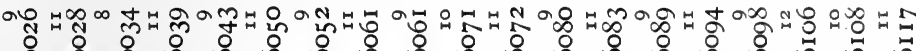

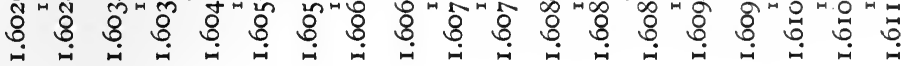

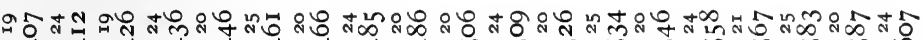
孛

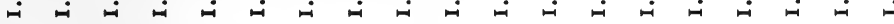

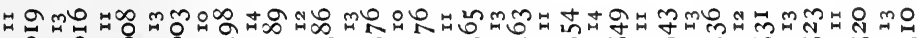

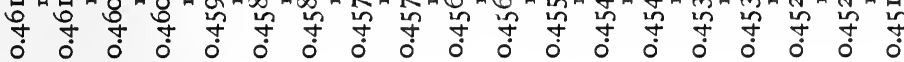

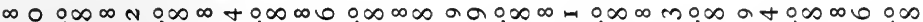
茂

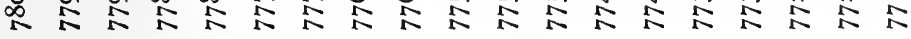

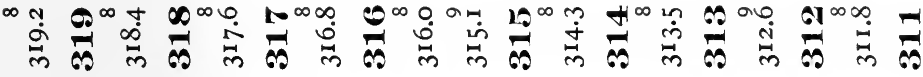

\section{क}

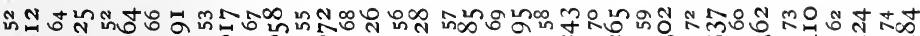

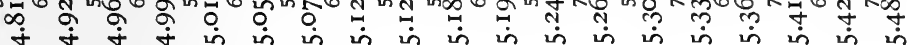

N

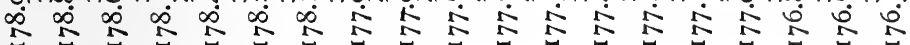
可

๑)

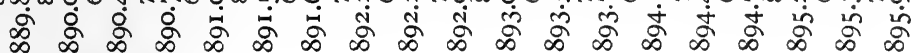
แ ஸ

○ $\infty$ t ஓं

$\infty$ " m a O

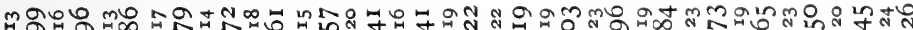

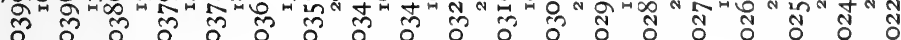
ஸே: 


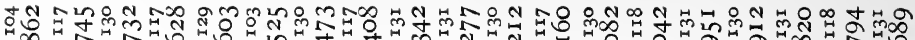

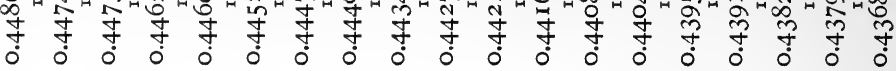

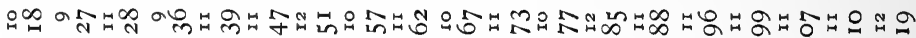
ర్ర

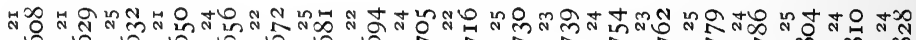

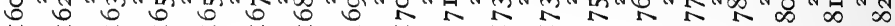

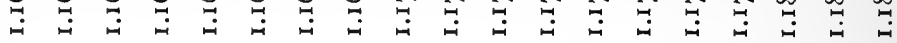

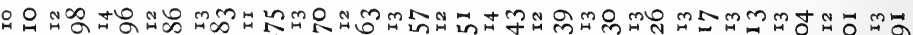

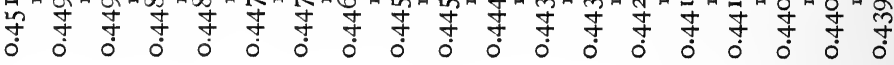

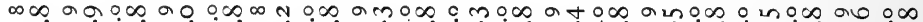
R $N$ N

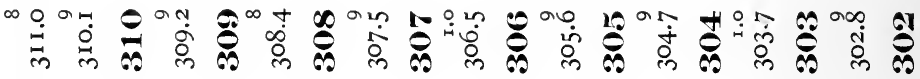

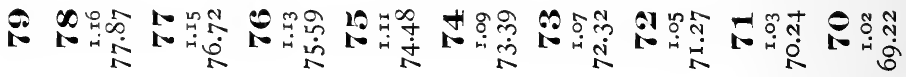

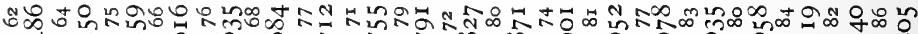

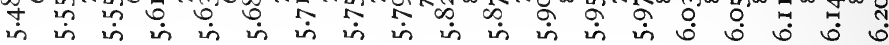

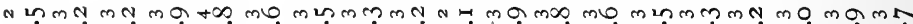

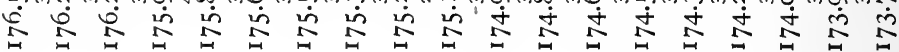

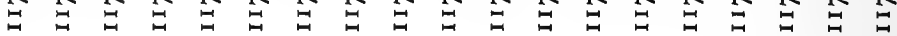

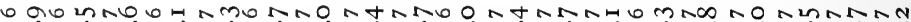
வ

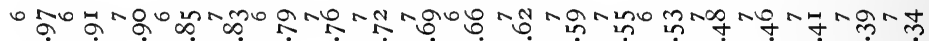

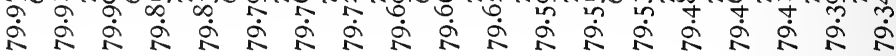

b a

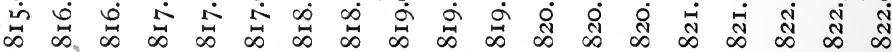

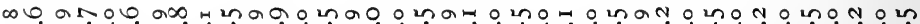
ᄋ

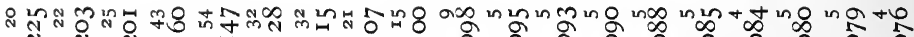

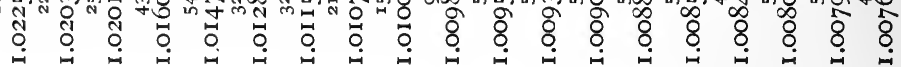




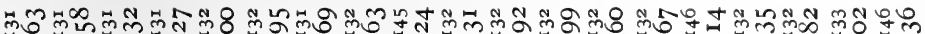

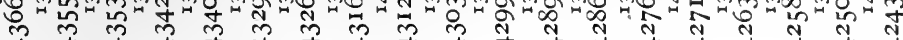

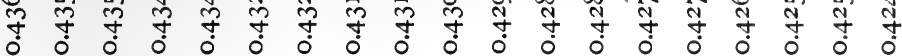

펴요

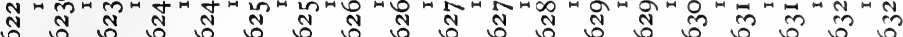
ర్ర

+

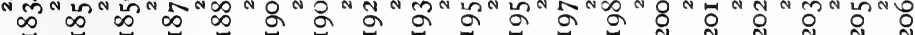

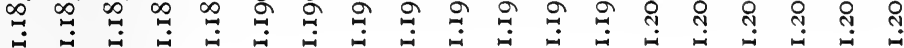

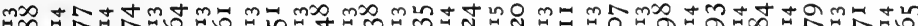

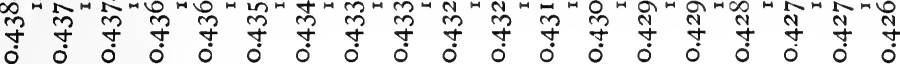

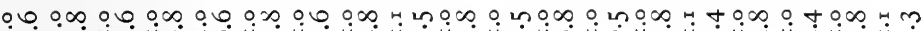

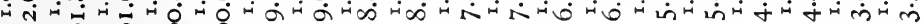
员

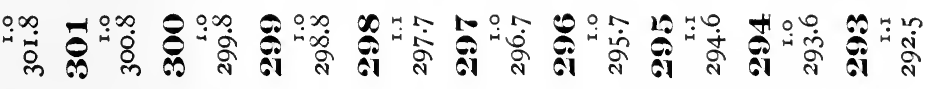

$$
\text { ๑ }
$$

め ஸे

mo m+mmmm+Q+N

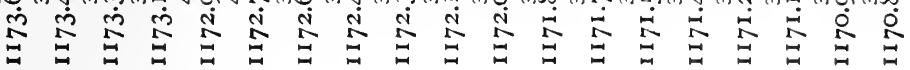

ヘナヘのN" N

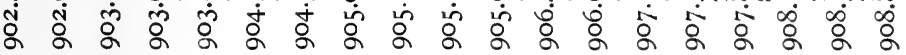

ஜ

N

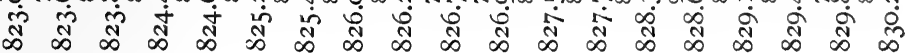

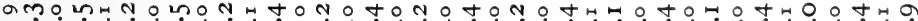

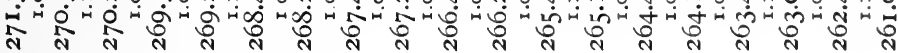

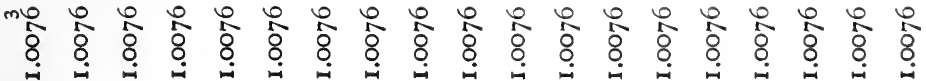




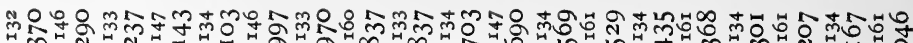
(2) ป

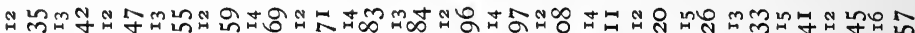

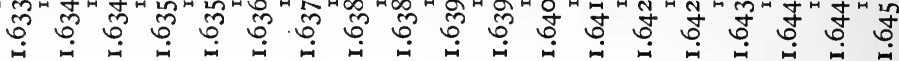

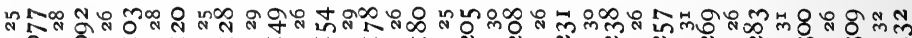

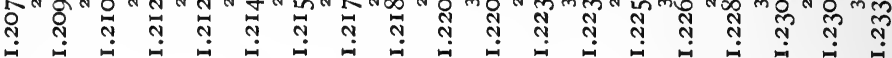

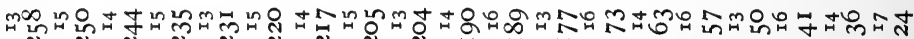

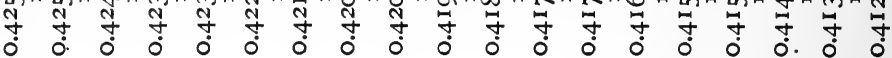

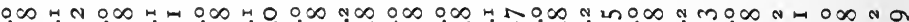

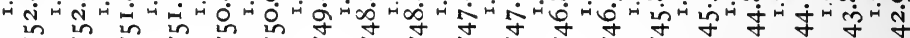

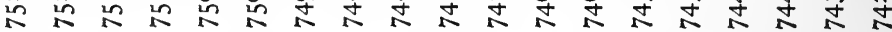

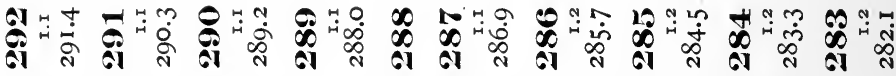

๙ป็

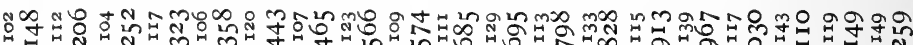

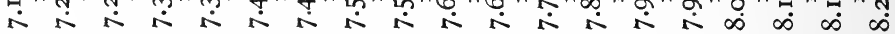

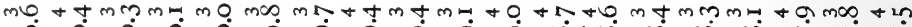
员

N

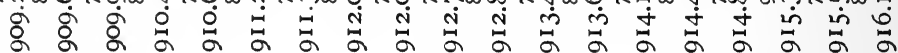

$\infty$ 군

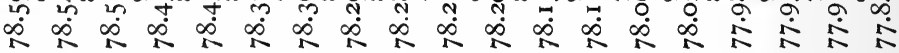

$\infty 0_{\infty} 0,+a 0_{\infty} N \infty$ TNO

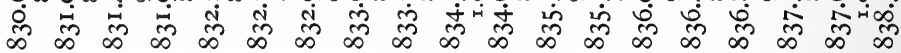

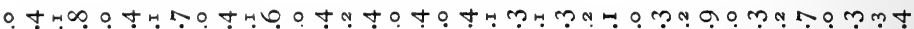
मூ

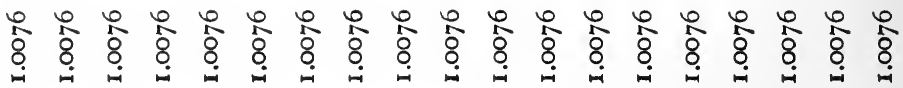




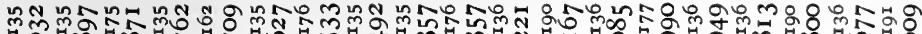

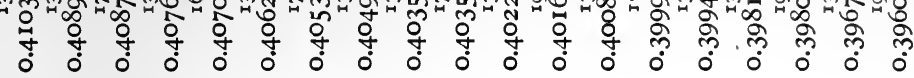

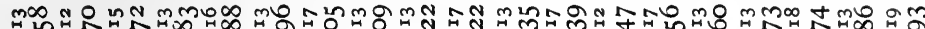

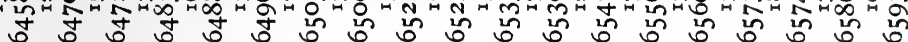

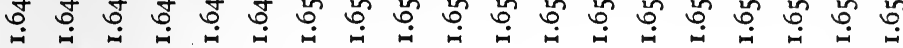

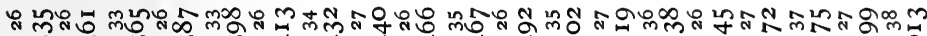

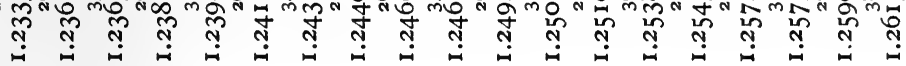

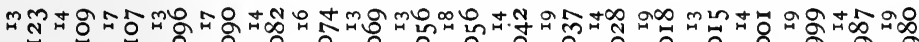

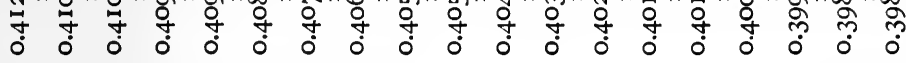

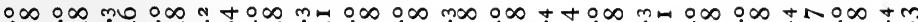

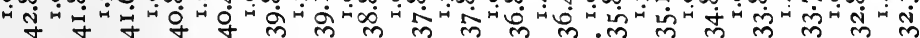

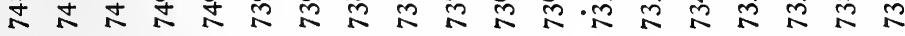

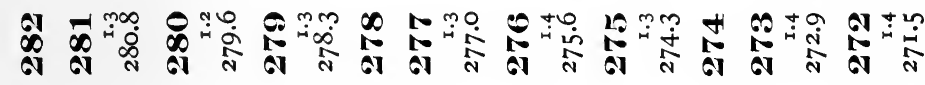

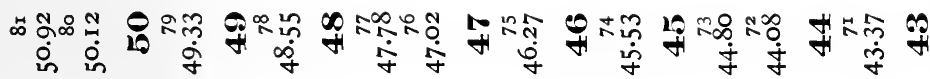

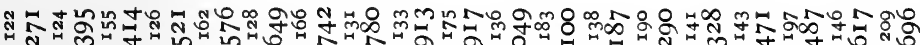
ஸ

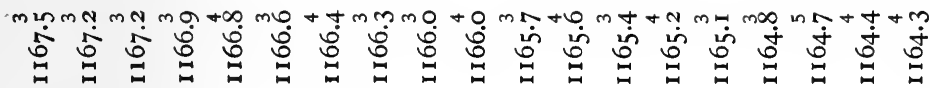

N" NG90 No a 0

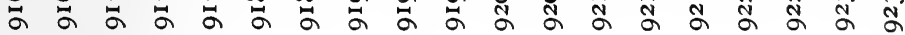

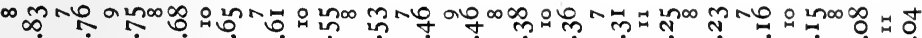

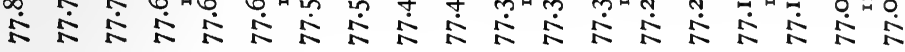

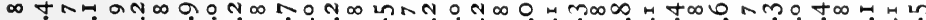

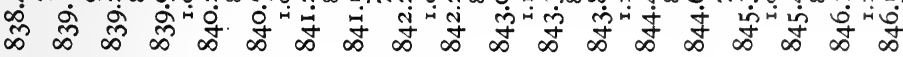

o mom मі㇒

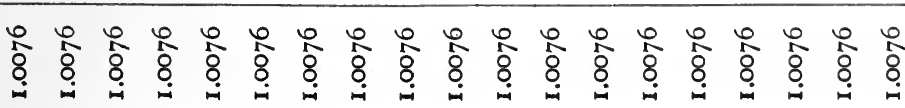




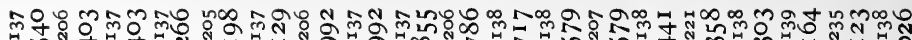

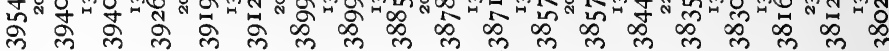

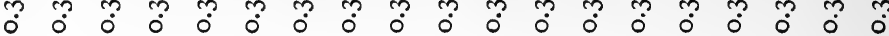

m 苗

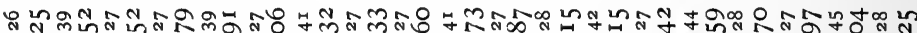
ชุ กุ

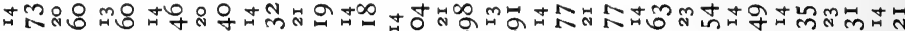
ूूरूे ஸे

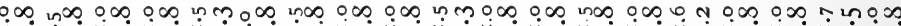
मேं $\mathbb{N} N \mathbb{N} N \mathbb{N} N \mathbb{N} N \mathbb{N} N \mathbb{N} N$

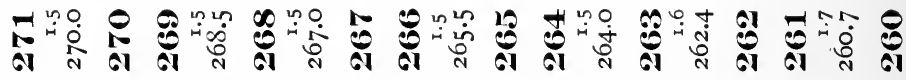

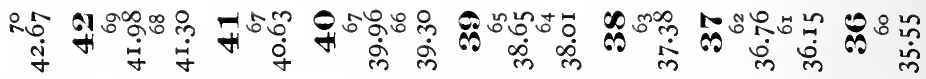
वणำ

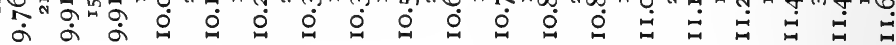

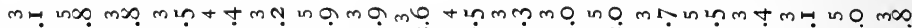
苛

n

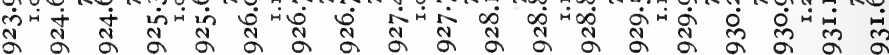

ヘே N

$\infty$ ดm

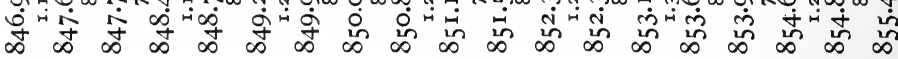

o

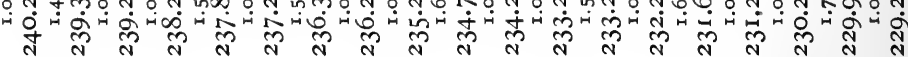

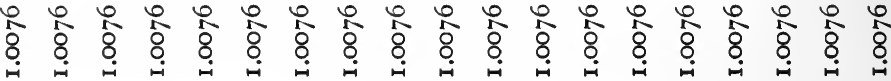




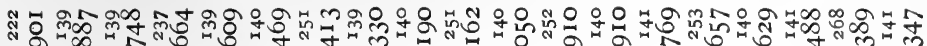

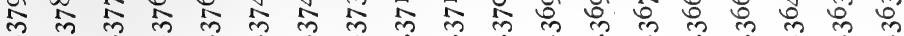

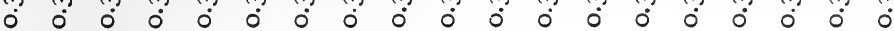

๘ है 0 है

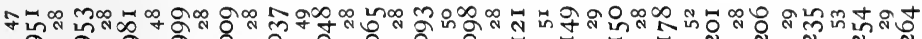

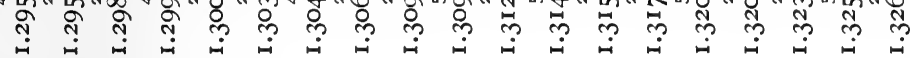

๓⿻口卄.

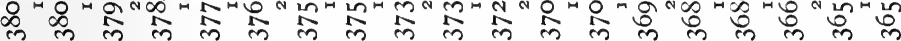

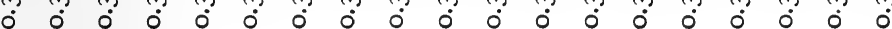

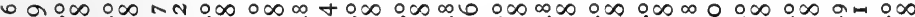

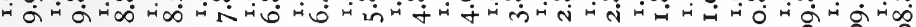

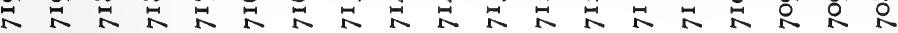

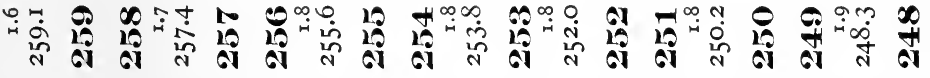

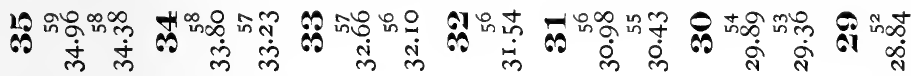

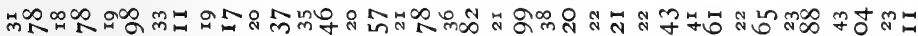

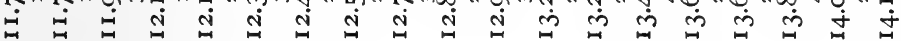

ம

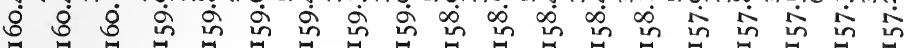

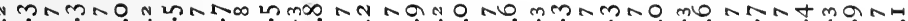

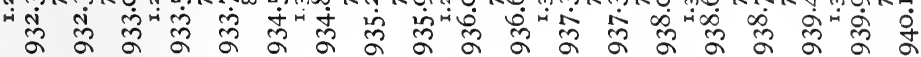

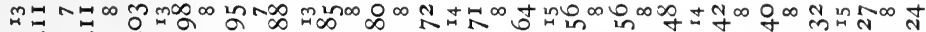

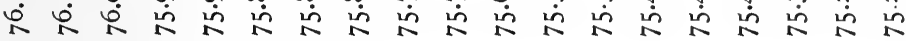

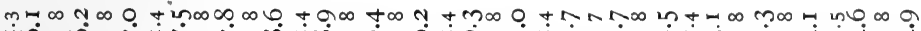

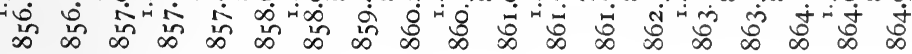

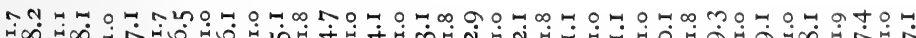

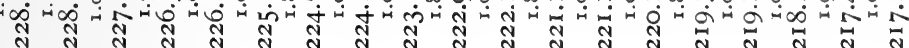

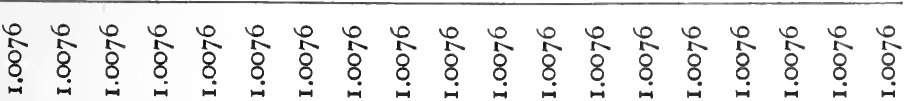




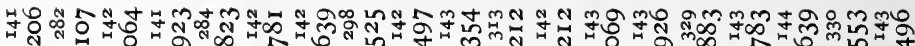
రु

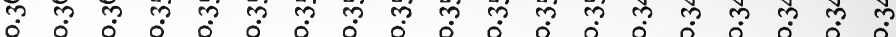

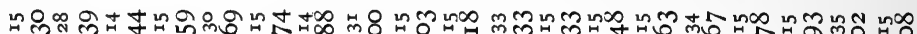

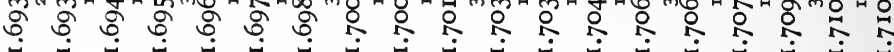

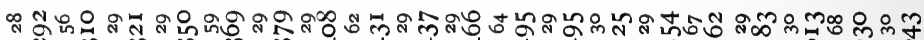

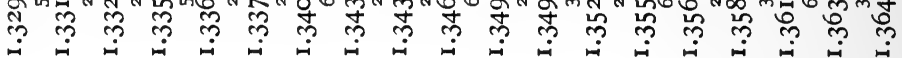

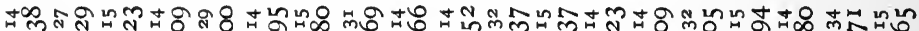

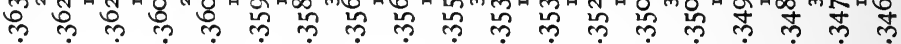

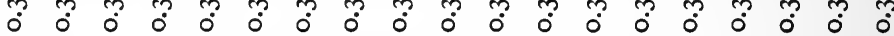

$0 \infty$ 무

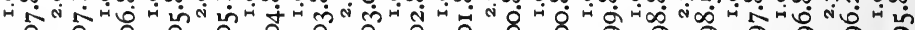

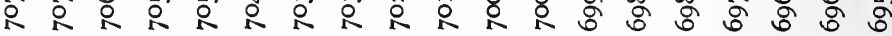

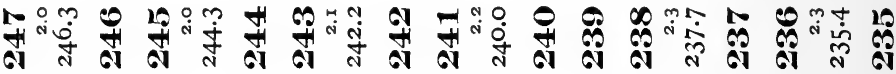

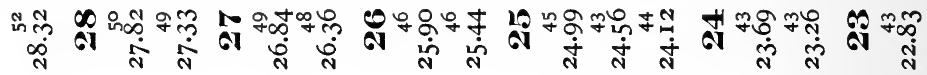

๓

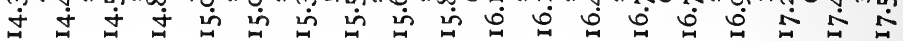

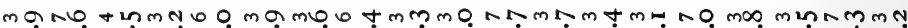

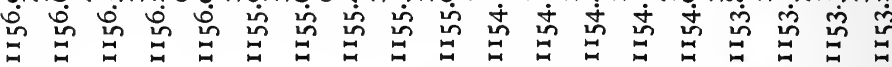

N ‡ँ

$\infty$ 유 采

N மூنூ

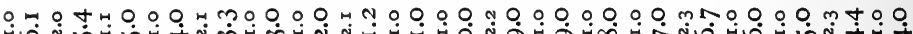

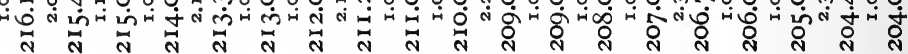

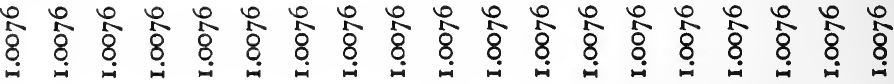




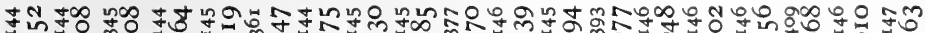

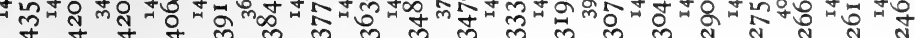
से

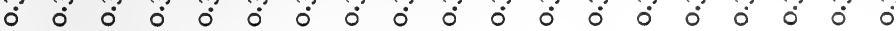

꿉

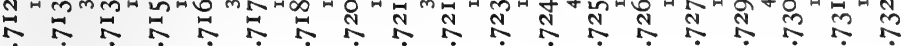

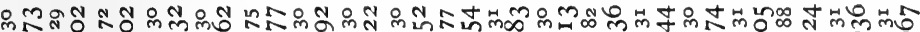

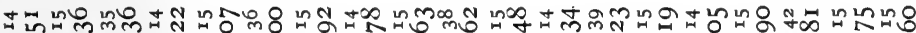
से ले से से ले ले $\begin{array}{lllllllllllllllllll}\circ & 0 & 0 & 0 & 0 & 0 & 0 & 0 & 0 & 0 & 0 & 0 & 0 & 0 & 0 & 0 & 0 & 0 & 0\end{array}$

$0 \infty, \infty+\infty \quad \infty \infty, \infty$ n मूँ

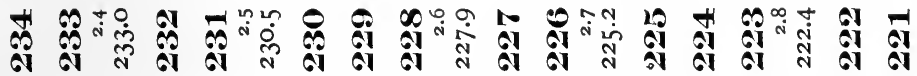

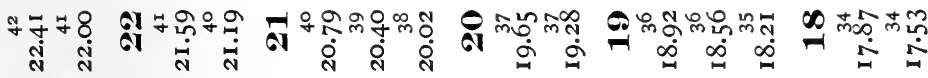

"

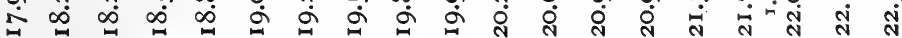

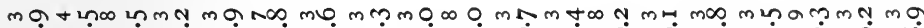
in in

N GNG No M ด

$\infty$ N $\infty$ I

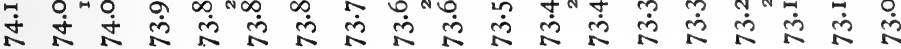

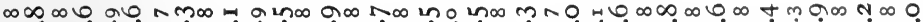
采

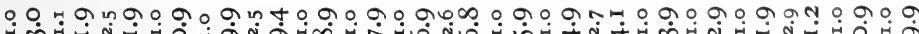

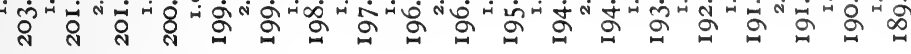

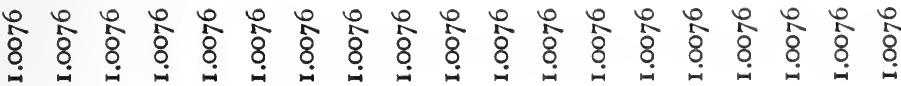




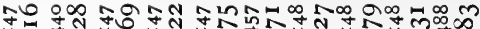

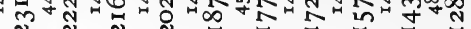
लै ले लै

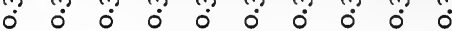
$\infty m \infty$ a $+\infty 6 m$ añ

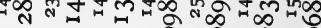
$m$ m $~ ल 0$ ठ

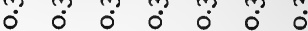

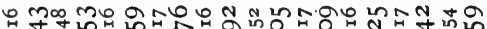

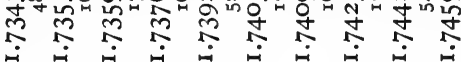

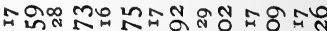

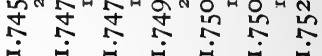

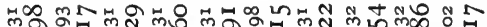
立

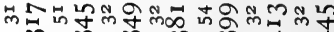

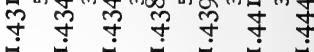

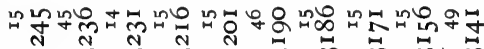

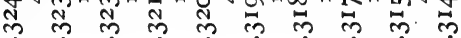

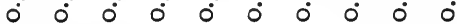

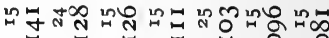

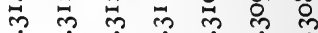

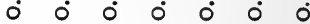

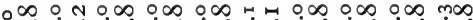

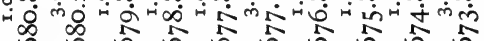

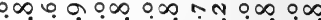
miñ

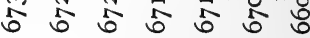

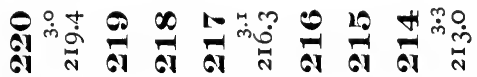

a) $\underset{\sim}{\stackrel{n}{N}}$ का

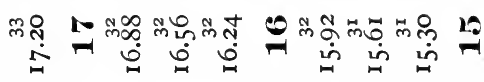

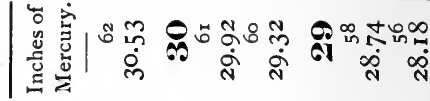

ส กे ले ले ले लें

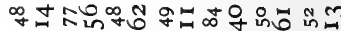
กั

mo atmmmo mNaนnmtmm mo o un

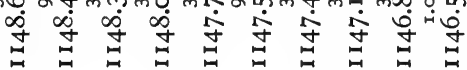

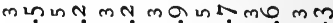
吕

NNMM ऊूं

ヘN

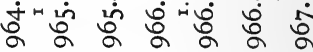

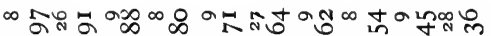

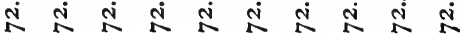

aำ भू i $\stackrel{N}{N} \stackrel{N}{N} \stackrel{N}{N} \stackrel{N}{N}$

$\infty \infty+? \infty 0,+\infty$ ก

ஸ

ஸ்

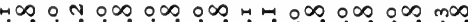
نळ

$0 \infty 0$ O $0 \infty \circ \infty N N \circ \infty \circ \infty$ 必宓边

$\begin{array}{llllllllll}0 & 0 & 0 & 0 & 0 & 0 & 0 & 0 & 0 & 0 \\ 0 & 0 & 0 & 1 & 1 & 1 & 1 & 1 & 1 & 1 \\ 0 & 0 & 8 & 8 & 0 & 0 & 0 & 0 & 0 & 0 \\ -1 & -1 & 1 & 0 & 0 & 0 & 0 & 0 & 0\end{array}$

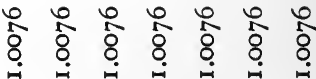


మై

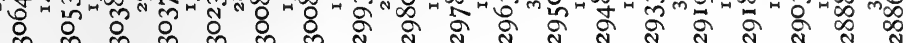
ర్ల

에

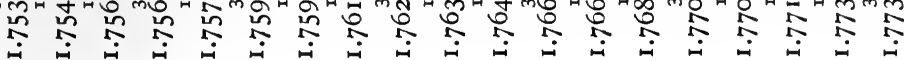

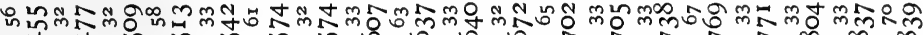

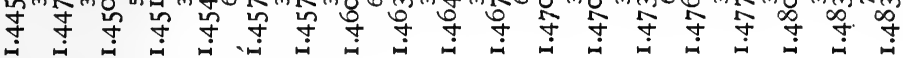

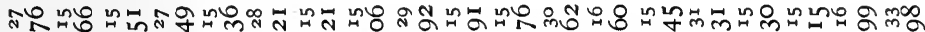

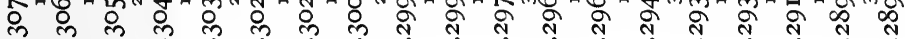
$\begin{array}{lllllllllllllllllll}0 & 0 & 0 & 0 & 0 & 0 & 0 & 0 & 0 & 0 & 0 & 0 & 0 & 0 & 0 & 0 & 0 & 0 & 0\end{array}$

† ت

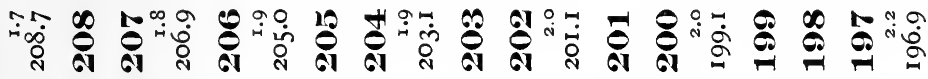

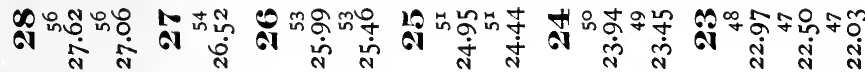

(2)

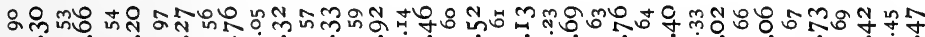
ळ

แก mO m 响

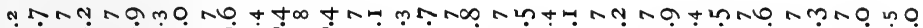
-

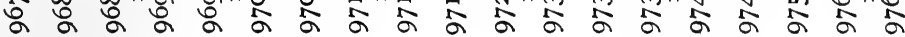

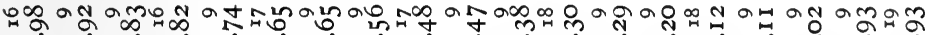

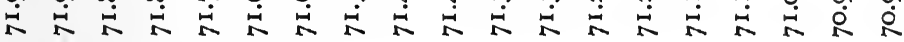

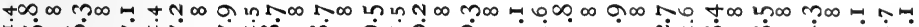
ஓ இ

$\infty+0 \infty$ r

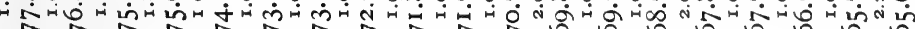

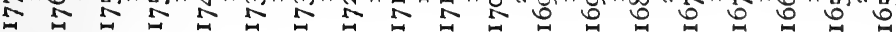

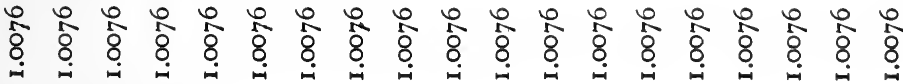




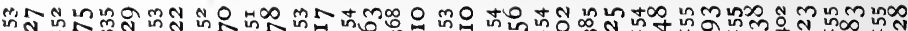
Nस्म

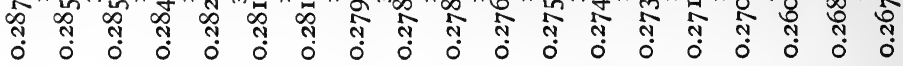

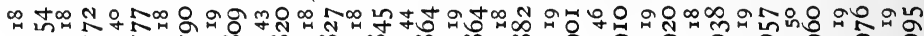

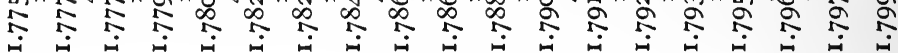

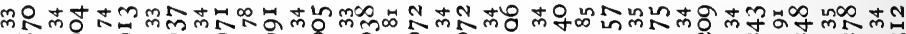

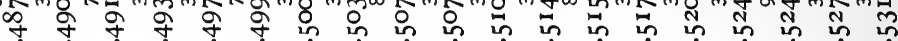

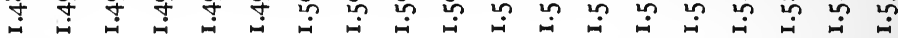

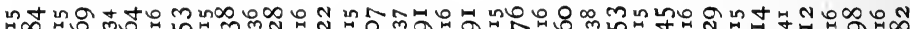

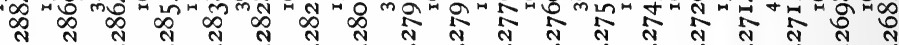

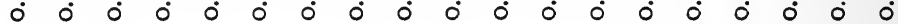

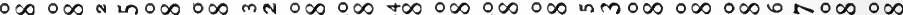

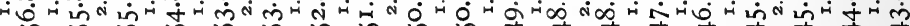
ஸூ

\section{๑) बें}

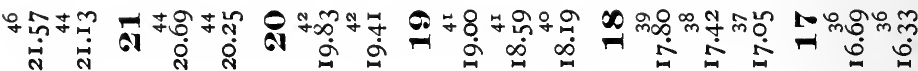

푼 ம

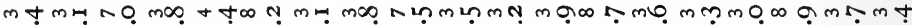

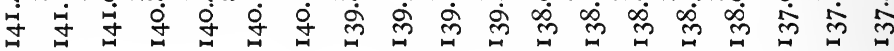

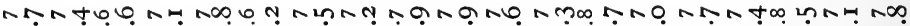

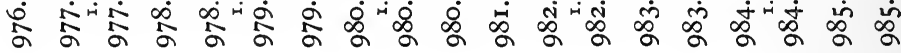
बオ

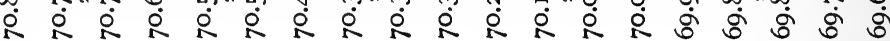
s

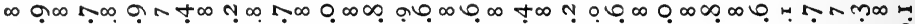

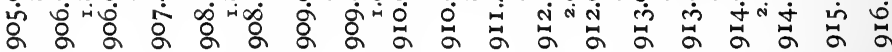

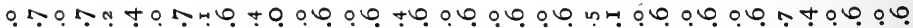

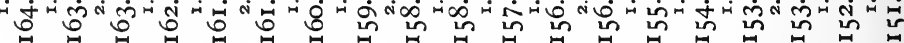

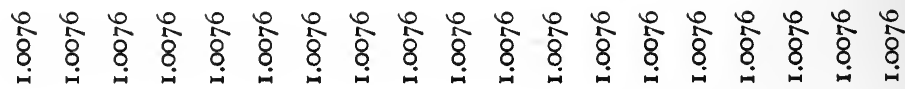




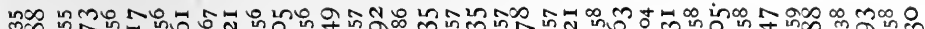
舟行 กุ.

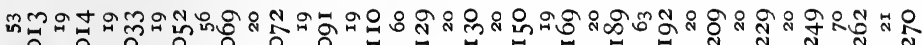
ర

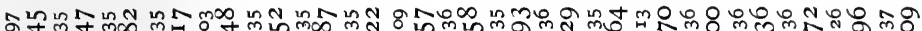
'

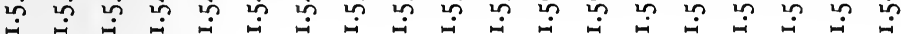

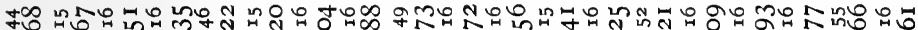

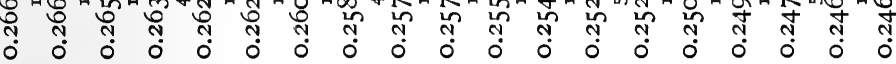

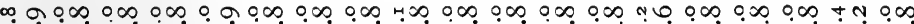

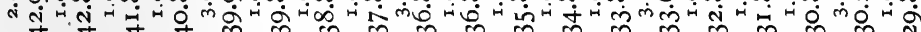

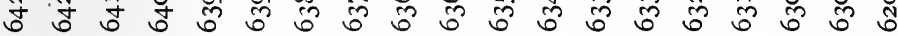

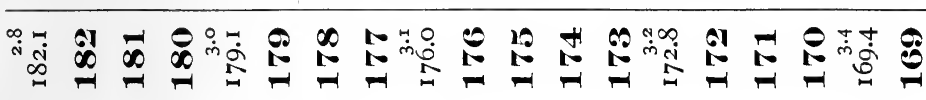

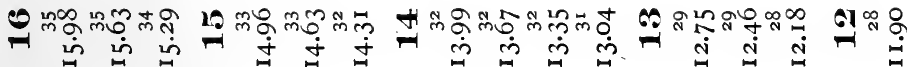

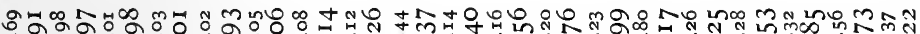

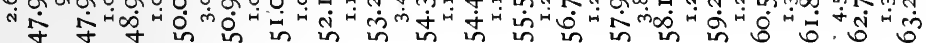

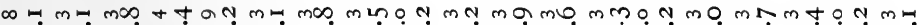

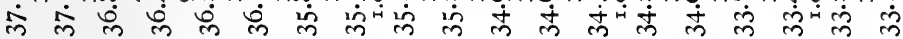

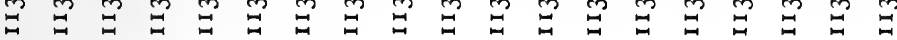

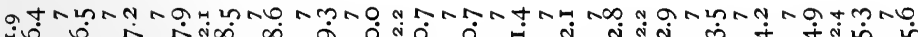
iூ

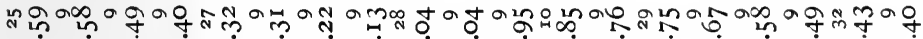

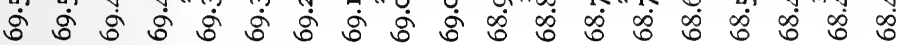

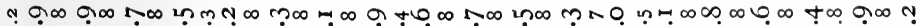
ง

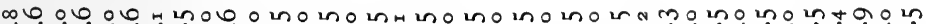

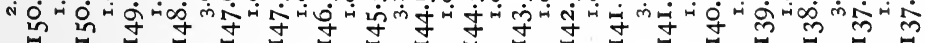

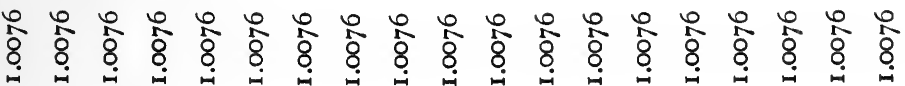




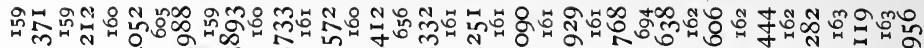
4
4
4

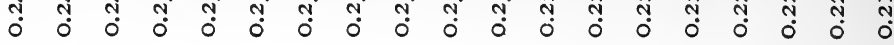

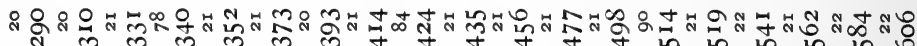
闻

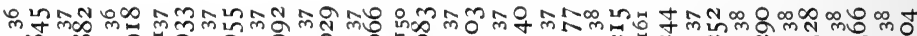

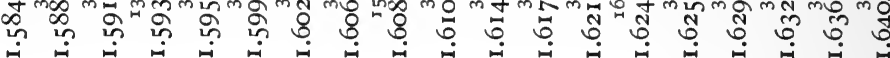

누웅

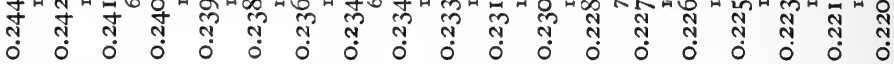

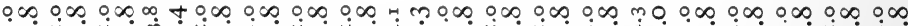

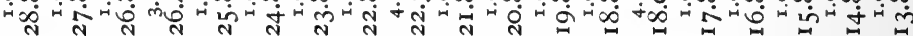

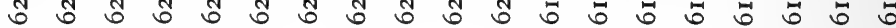

\section{๑}

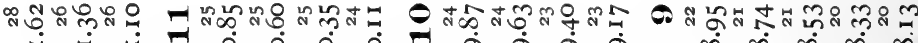
业

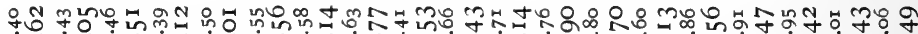
过鸩

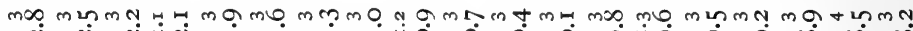
ஸे

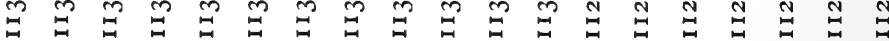

ヘッnO ஜ

음구

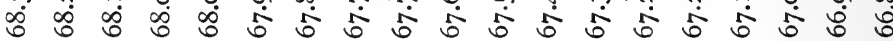

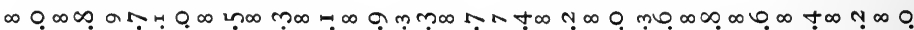
ஸे

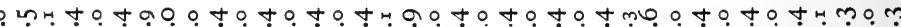
मூ

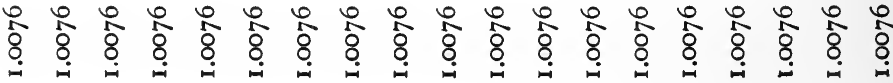




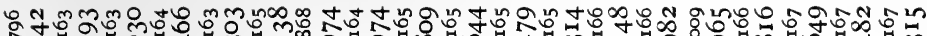
ส

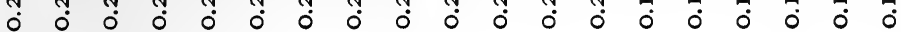

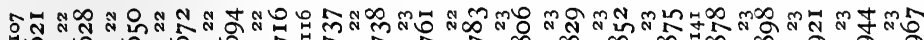

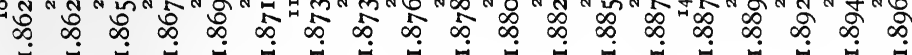

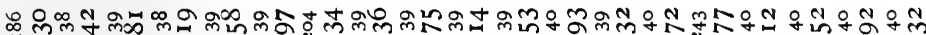

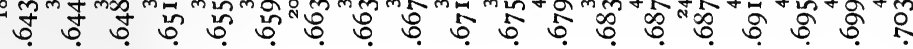

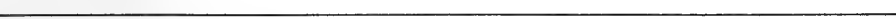

แ สุ

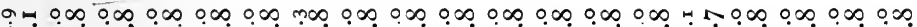
ஏூ

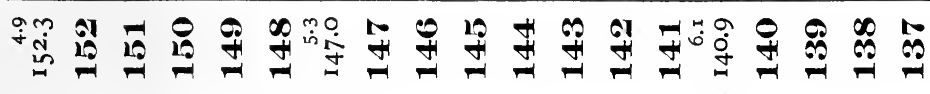

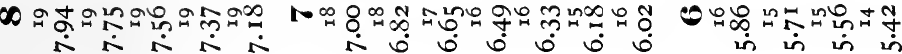

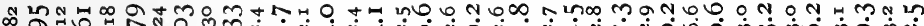

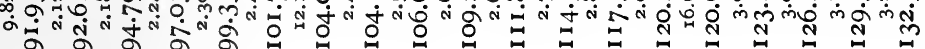

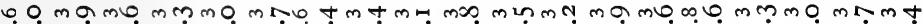

峁

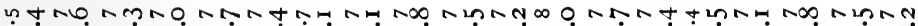

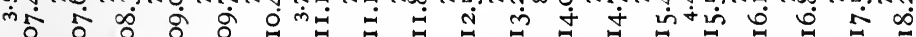

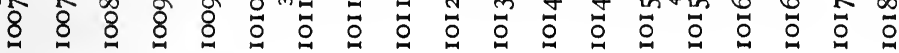

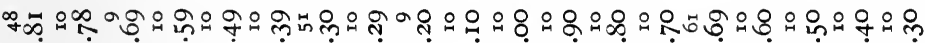
ஜி ஜீ

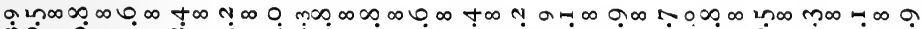
ஓं

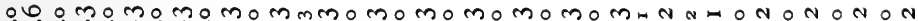
ทं

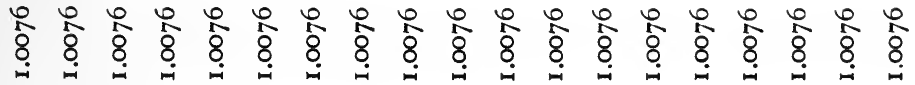




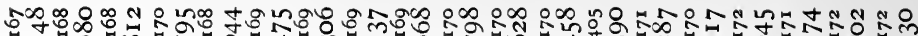
ద ○ 0 :

สำ

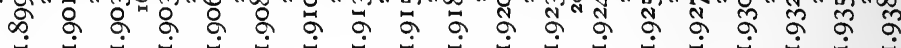

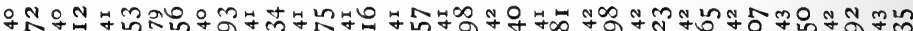
○一

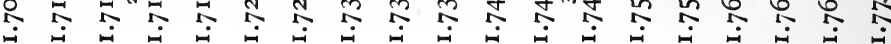

윤 の'

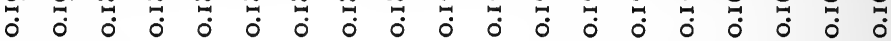

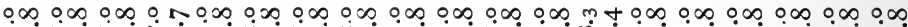
نூ

\section{ஸे}

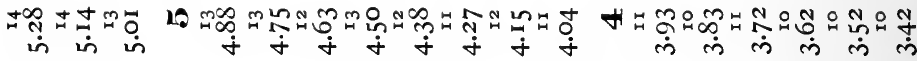

$m \infty+20 \infty$ й mं

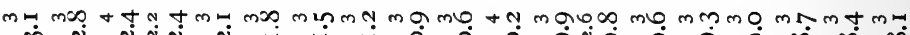
営

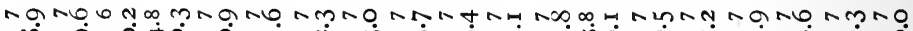
葄

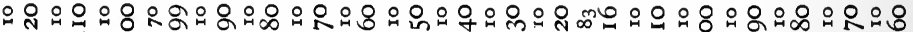
ம்

$\infty$ Non nn un 의

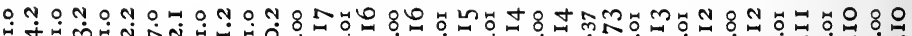
过

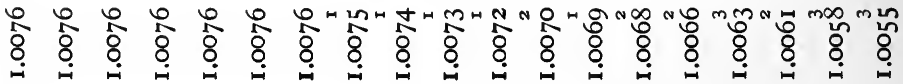


술 mนN

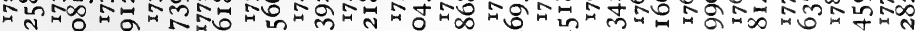
ช유

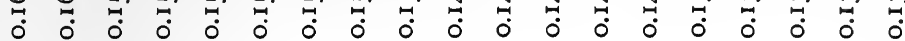

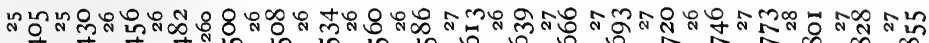

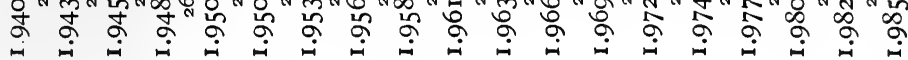

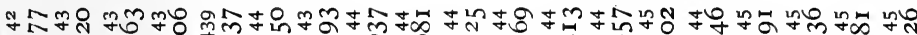
స

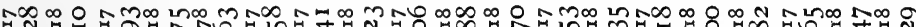

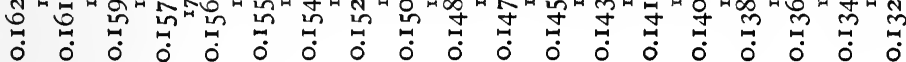

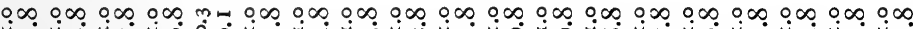
ல்

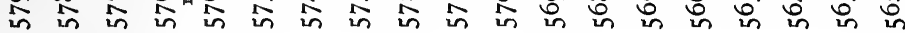

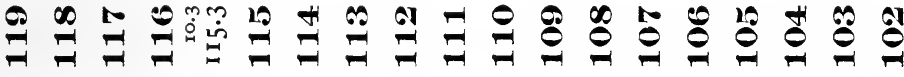

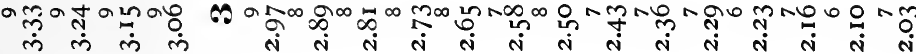

in बio un mo mo $\mathrm{N}$ in ம்

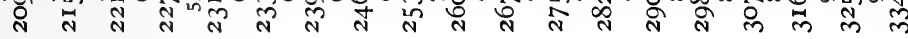

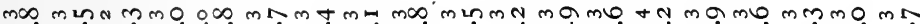

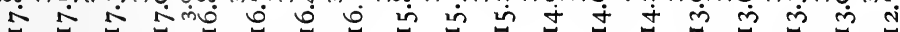

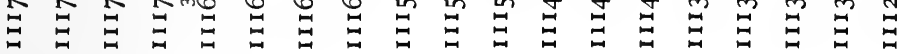

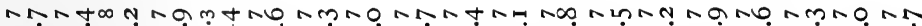
윰

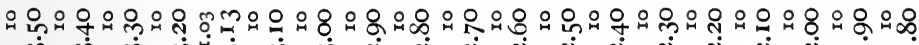

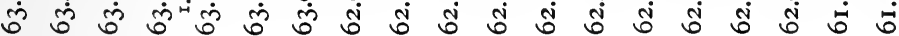

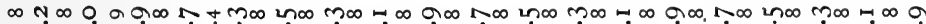

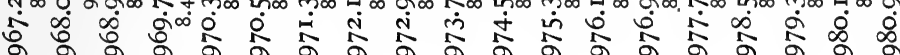

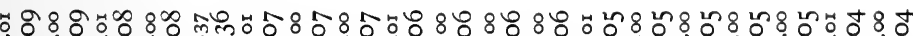

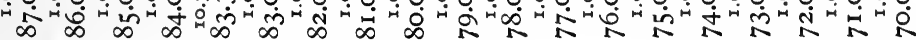

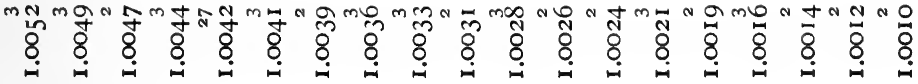




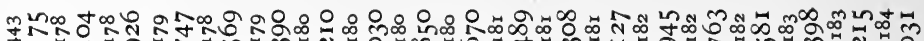
a m

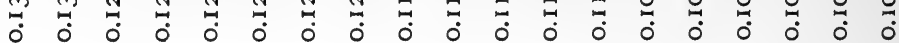

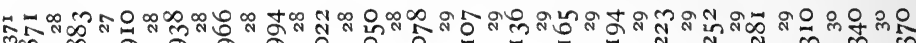
品

以

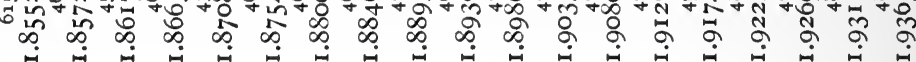

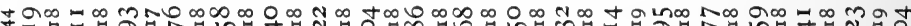
苗

a m

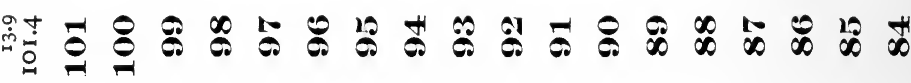

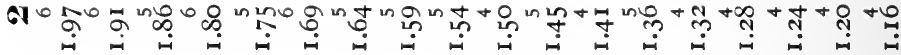

moюm

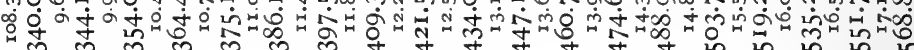

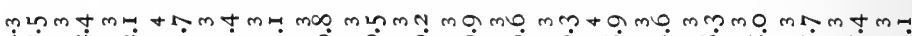

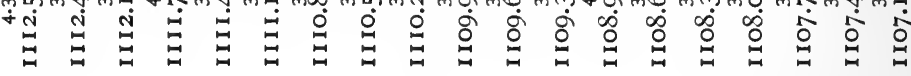

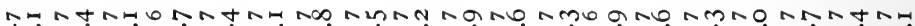

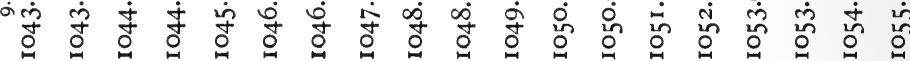

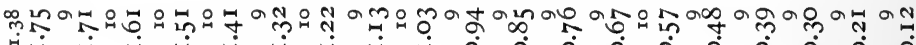
تే

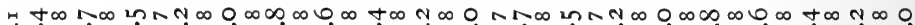

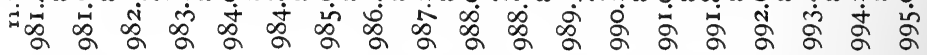

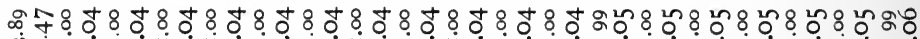
๓ं

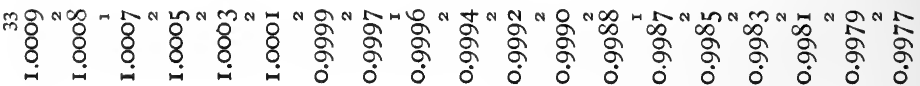





\section{A Laboratory Manual}

OF

\section{Physics and Applied Electricity.}

ARRANGED AND EDITED BY

EDWARD L. NICHOLS,

Professor of Physics in Cornell University.

IN TWO VOLUMES.

Vol. I. JUNIOR COURSE IN GENERAL PHYSICS.

BY

ERNEST MERRITT and FREDERICK J. ROGERS.

Cloth. \$3.00.

\section{Vol. II. SENIOR COURSES AND OUTLINE OF ADVANCED WORK.}

BY

GEORGE S. MOLER, FREDERICK BEDELL, HOMER J. HOTCHKISS, CHARLES P. MATTHEWS, and THE EDITOR.

Cloth. pp. 444. \$3.25.

The first volume, intended for beginners, affords explicit directions adapted to a modern laboratory, together with demonstrations and elementary statements of principles. It is assumed that the student possesses some knowledge of analytical geometry and of the calculus. In the second volume more is left to the individual effort and to the maturer intelligence of the practicant.

A large proportion of the students for whom primarily this Manual is intended, are preparing to become engineers, and especial attention has been devoted to the needs of that class of readers. In Vol. II., especially, a considerable amount of work in applied electricity, in photometry, and in heat has been introduced.

\section{COMMENTS.}

"The work as a whole cannot be too highly commended. Its brief outlines of the various experiments are very satisfactory, its descriptions of apparatus are excellent; its numerous suggestions are calculated to develop the thinking and reasoning powers of the student. The diagrams are carefully prepared, and its frequent citations of original sources of information are of the greatest value." - Street Railway Fournal.

"The work is clearly and concisely written, the fact that it is edited by Professor Nichols being a sufficient guarantee of merit." - Electrical Engineering.

"It will be a great aid to students. The notes of experiments and problems reveal much original work, and the book will be sure to commend itself to instructors." - San Francisco Chronicle.

\section{THE MACMILLAN COMPANY}

66 FIFTH AVENUE, NEW YORK 


\title{
THE ELEMENTS OF PHYSICS.
}

BY

\author{
EDWARD L. NICHOLS, B.S., Ph.D., \\ Professor of Physics in Cornell University,
}

AND

WILLIAM S. FRANKLIN, M.S.,

Professor of Physics and Electrical Engineering at the Iowa Agricultural College, Ames, Ia.

WITH NUMEROUS ILLUSTRATIONS.

Part I. In Three Volumes:

Vol. I. Mechanics and Heat . . Price \$r.50 net.

II. Electricity and Magnetism . "\$1.90 net.

III. Sound and Light . . . “\$1.50 net.

It has been written with a view to providing a text-book which shall correspond with the increasing strength of the mathematical teaching in our university classes. In most of the existing text-books it appears to have been assumed that the student possesses so scanty a mathematical knowledge that he cannot understand the natural language of physics, i.e., the language of the calculus. Some authors, on the other hand, have assumed a degree of mathematical training such that their work is unreadable for nearly all undergraduates.

The present writers having had occasion to teach large classec, the members of which were acquainted with the elementary principles of the calculus, have sorely felt the need of a text-book adapted to their students. The present work is an attempt on their part to supply this want. It is believed that in very many institutions a similar condition of affairs exists, and that there is a demand for a work of a grade intermediate between that of the existing elementary texts and the advanced manuals of physics.

No attempt has been made in this work to produce a complete manual or compendium of experimental physics. The book is planned to be used in connection with illustrated lectures, in the course of which the phenomena are demonstrated and described. The authors have accordingly confined themselves to a statement of principles, leaving the lecturer to bring to notice the phenomena based upon them. In stating these principles, free use has been made of the calculus, but no demand has been made upon the student beyond that supplied by the ordinary elementary college courses on this subject.

Certain parts of physics contain real and unavoidable difficulties. These have not been slurred over, nor have those portions of the subject which contain them been omitted. It has been thought more serviceable to the student and to the teacher who may have occasion to use the book to face such difficulties frankly, reducing the statements involving them to the simplest form which is compatible with accuracy.

In a word, the Elements of Physics is a book which has been written for use in such institutions as give their undergraduates a reasonably good mathematical training. It is intended for teachers who desire to treat their subject as an exact science, and who are prepared to supplement the brief subject-matter of the text by demonstration, illustration, and discussion drawn from the fund of their own knowledge.

\section{THE MACMILLAN COMPANY}




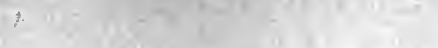

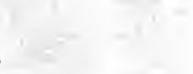




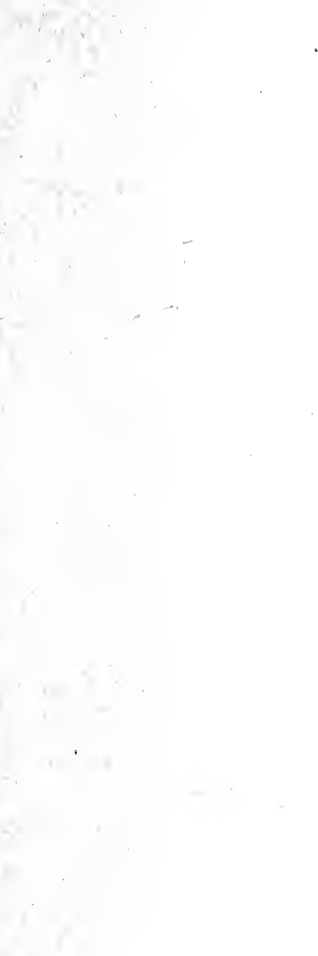

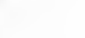

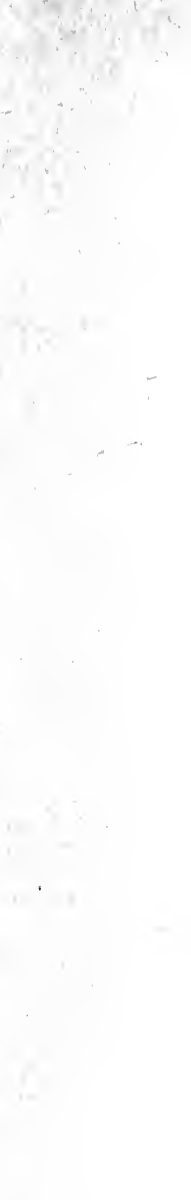





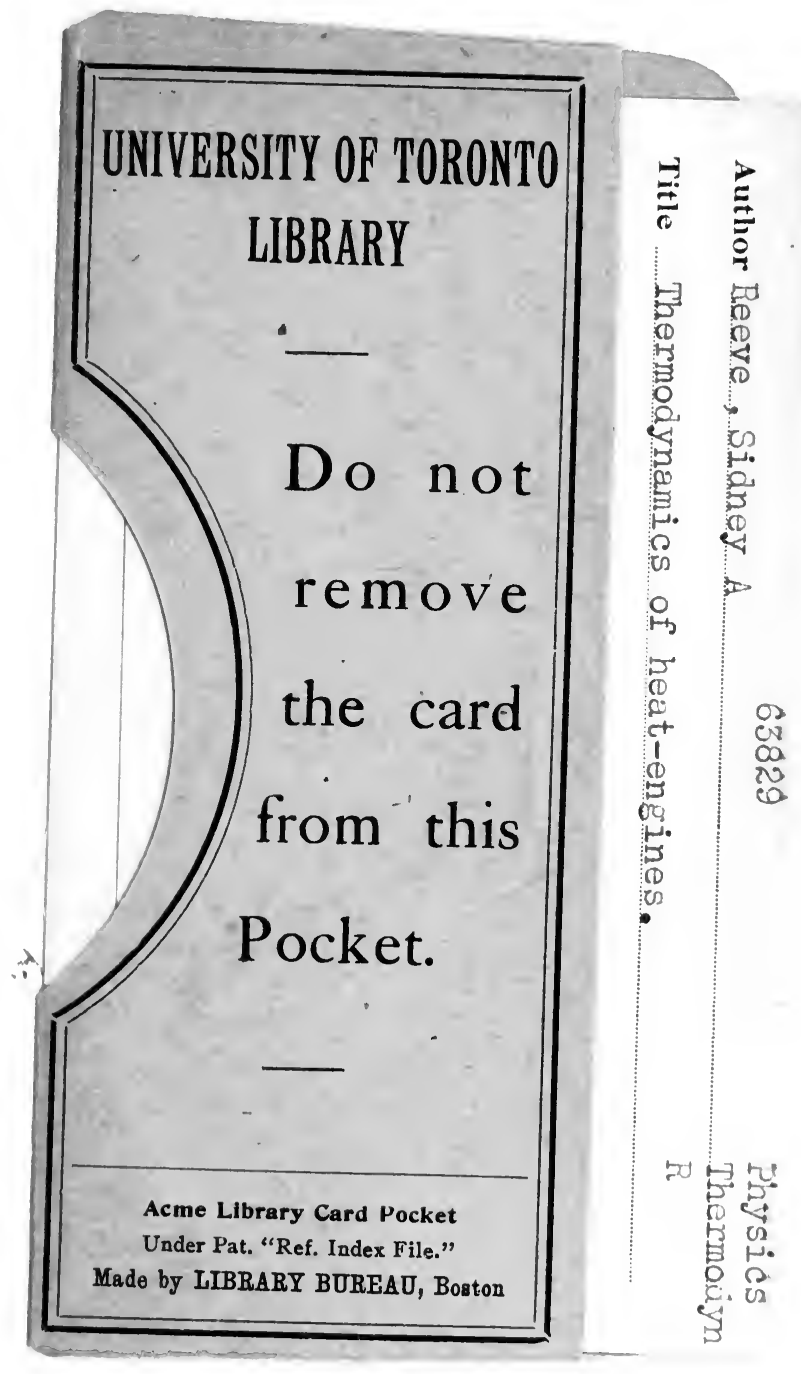


F,

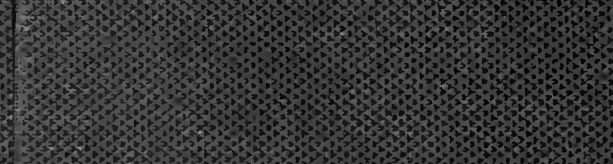

3.

mos.

3. 30

X.,

ind

, n.t.

3.

1. 3.

A.

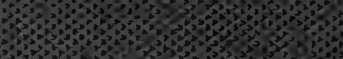

(3) 